\title{
Development and Validation of a Planar On-Deck Helicopter Manoeuvring Simulation
}

\author{
by \\ Darren P. Linn, B.Eng. \\ Carleton University \\ A thesis submitted to \\ the Faculty of Graduate Studies and Research \\ in partial fulfillment of \\ the requirements for the degree of \\ Master of Applied Science \\ Ottawa-Carleton Institute for \\ Mechanical and Aerospace Engineering
}

Department of

Mechanical and Aerospace Engineering

Carleton University

Ottawa, Ontario

December 2003

(c) Copyright

2003 - Darren R. Linn 


\author{
National Library \\ of Canada \\ Acquisitions and \\ Bibliographic Services \\ 395 Wellington Street \\ Ottawa ON K1A ON4 \\ Canada
}

Bibliothèque nationale

du Canada

Acquisisitons et services bibliographiques

395 , rue Wellington Ottawa ON K1A ON4 Canada
Your file Votre référence ISBN: 0-612-89851-2

Ourfile Notre référence ISBN: 0-612-89851-2
The author has granted a nonexclusive licence allowing the National Library of Canada to reproduce, loan, distribute or sell copies of this thesis in microform, paper or electronic formats.

The author retains ownership of the copyright in this thesis. Neither the thesis nor substantial extracts from it may be printed or otherwise reproduced without the author's permission.
L'auteur a accordé une licence non exclusive permettant à la Bibliothèque nationale du Canada de reproduire, prêter, distribuer ou vendre des copies de cette thèse sous la forme de microfiche/film, de reproduction sur papier ou sur format électronique.

L'auteur conserve la propriété du droit d'auteur qui protège cette thèse. $\mathrm{Ni}$ la thèse ni des extraits substantiels de celle-ci ne doivent être imprimés ou aturement reproduits sans son autorisation.
In compliance with the Canadian Privacy Act some supporting forms may have been removed from this dissertation.

While these forms may be included in the document page count, their removal does not represent any loss of content from the dissertation.
Conformément à la loi canadienne sur la protection de la vie privée, quelques formulaires secondaires ont été enlevés de ce manuscrit.

Bien que ces formulaires aient inclus dans la pagination, il n'y aura aucun contenu manquant. 


\section{Abstract}

An interactive planar helicopter manoeuvring simulation, HeliMan, has been developed to model on-deck helicopter handling using shipboard aircraft handling equipment with emphasis on the Indal Technologies Inc. (ITI) Aircraft/Ship Integrated Secure and Traverse (ASIST) system. HeliMan uses a proven transient three-degree-of-freedom tire model for each tire and incorporates these behaviours into a four-degree-of-freedom helicopter model, which includes an option for a castorable or steerable nose or tail wheel. When modelling a slow-moving tire as in this application, accurate methods for modelling relaxation length and cornering force generation are needed, making the model the most complex part of the simulation.

The simulation was verified and full-scale validation testing was performed. Validation testing was performed using the Integrated Test Facility (ITF), available at ITI, that is equipped with a full-scale Dead-Load Test Vehicle (DLTV) and ASIST system. A number of simple manoevres were performed to validate specific aspects of the simulation. The DLTV and ASIST system were instrumented to collect kinematic and force data, while the DLTV was being manipulated. This data included: the Rapid Securing Device (RSD) lateral and longitudinal position; the force acting on the securing probe; the orientation of the DLTV and its castor wheel; and the lateral tire deflections. The DLTV's position and orientation were measured manually periodically during the manoeuvre. The results are compared and discussed with results generated by HeliMan.

It is shown that the HeliMan simulation captures the underlying dynamics of the shipboard helicopter manoeuvring process. Full-scale validation data shows that in many cases, the simulation is able to reproduce closely the measured data. The effect of tire side loading on longitudinal rolling resistance has been identified as the most probable cause for differences between simulated and measured results in a number of validation cases. 


\section{Acknowledgements}

First and foremost I would like to thank my thesis supervisor Dr. Robert Langlois for his guidance, support and patience throughout this project. His willingness to make himself available at any time was invaluable, to the possible detriment of his own work. Again, I thank you.

Valuable contributions were also made by Michael LaRosa and Mark Devlin at Indal Technologies Inc. in answering questions about the inner workings of the ASIST system; Michael Brown for setting up the joystick interface; Mandy Feldman and the rest of Dr. Langlois' Applied Dynamics Group, including honourary members, for listening to and critiquing my ideas; the departmental staff for making the bureaucratic red tape much easier to cut; and the boys of Jimmy's Garage for providing the ever-important leisure activities. Last, but certainly not least, the encouragement and patience that the love of my life, Elayne Duggan, has shown throughout this process is appreciated.

Finally, this work would not have been possible without the financial support provided by Indal Technologies Ine. for which Dr. Atef Tadros was the project consultant, Materials and Manufacturing Ontario for which Carole Champion was the project liaison and the Department of Mechanical and Aerospace Engineering. 
Dedicated to my family,

for always encouraging me to take my own path. 


\section{List of Symbols}

\section{Subscripts:}

$x, y, z \quad x$ component, $y$ component, $z$ component

\section{Superscripts:}

$\begin{array}{ll}c & \text { Castor assembly frame of reference } \\ g & \text { Global frame of reference } \\ h & \text { Helicopter frame of reference } \\ i & \text { Initial value of variable } \\ l & \text { Local frame of reference } \\ m & \text { Manufacturer's helicopter frame of reference } \\ m a x, \min & \text { A maximum or minimum value } \\ t & \text { Tire frame of reference } \\ , " \prime & \text { An adjusted value within a matrix or vector }\end{array}$

Overscripts:

$\begin{array}{ll}. & \text { (dot) First time derivative } \\ . & \text { (double-dot) Second time derivative } \\ & \text { Vector }\end{array}$

Variables:

$A_{e x} \quad$ Equivalent cross-sectional area of the helicopter for the plane perpendicular to the $\mathrm{x}$ plane

$A_{e y} \quad$ Equivalent cross-sectional area of the helicopter for the plane perpendicular to the $y$ plane 


$\begin{array}{ll}C_{B} & \text { Coefficient of viscous damping at the castor pivot joint } \\ C_{f i x} & \text { Torsional damping coefficient used to fix the castor assembly } \\ C_{P} & \text { Probe damping coefficient } \\ C_{t_{y}} & \text { Tire lateral damping coefficient } \\ C_{t z} & \text { Vertical tire force constant dependent on the tire type } \\ D_{P s} & \text { Small probe deflection stiffness transition displacement } \\ d_{s s_{y}} & \text { Steady state lateral tire deflection for a given slip angle } \\ F_{a} & \text { Friction force between axle and wheel } \\ F_{r r} & \text { Rolling resistance force } \\ F_{s s_{y}} & \text { Steady state lateral tire force for a given slip angle } \\ F_{\kappa} & \text { Force acting on the castor tires due to the kingpin inclination angle } \\ h & \text { Height between castor pivot point and wheel pivoting arm perpendicular } \\ h_{C} & \text { to the ground } \\ I_{C} & \text { Mass moment of inertia of the castor assembly body } \\ I_{H} & \text { Mass moment of inertia of the main helicopter body } \\ K_{f i x} & \text { Torsional stiffness used to fix the castor assembly } \\ K_{P} & \text { Probe stiffness coefficient } \\ K_{P s} & \text { Third molion displacement probe stiffness coefficient } \\ k_{h} & \text { Radius of gyration of the helicopter } \\ E_{3} & \text { Distance perpendicular to the pivot axis to a plane perpendicular to } \\ L_{h} & \text { the ground }\end{array}$




\begin{tabular}{|c|c|}
\hline$m_{C}$ & Mass of the castor assembly body \\
\hline$m_{H}$ & Mass of the main helicopter body \\
\hline$N$ & Cornering power of tire \\
\hline$n$ & Polytropic exponent \\
\hline$P$ & Instantaneous tire pressure \\
\hline$p_{l}$ & Projected length \\
\hline$P_{o}$ & Tire inflation pressure at zero vertical load (gauge) \\
\hline$P_{o a}$ & Tire inflation pressure at zero vertical load (absolute) \\
\hline$P_{r}$ & Rated tire pressure \\
\hline$q_{1}$ & Helicopter X position in global coordinates \\
\hline$q_{2}$ & Helicopter $Y$ position in global coordinates \\
\hline$q_{3}$ & Helicopter orientation in global coordinates \\
\hline$q_{4}$ & Castor assembly orientation relative to helicopter coordinate system \\
\hline$r_{a}$ & Radius of the tire axle \\
\hline$r_{\dot{i}}$ & Radius of tire \\
\hline$r_{\kappa}$ & Absolute distance between castor pivot axis and the wheel axle \\
\hline$r_{\mu}$ & Radius of the kingpin \\
\hline$S_{t}$ & Tire shape constant \\
\hline$M_{B}$ & Sum of the reaction torques acting on the castor joint \\
\hline$T_{\mu}$ & Torque acting on the castor joint due to Coulomb friction \\
\hline$T_{v}$ & Torque acting on the castor joint due to viscous damping \\
\hline$w_{t}$ & Width of tire \\
\hline$X_{J}$ & Joystick $\mathrm{x}$ axis \\
\hline$Y_{J}$ & Joystick y axis \\
\hline$\alpha$ & Instantaneous angle of castor wheel due to the kingpin inclination \\
\hline$\alpha_{C}$ & Castor assembly angular acceleration \\
\hline
\end{tabular}




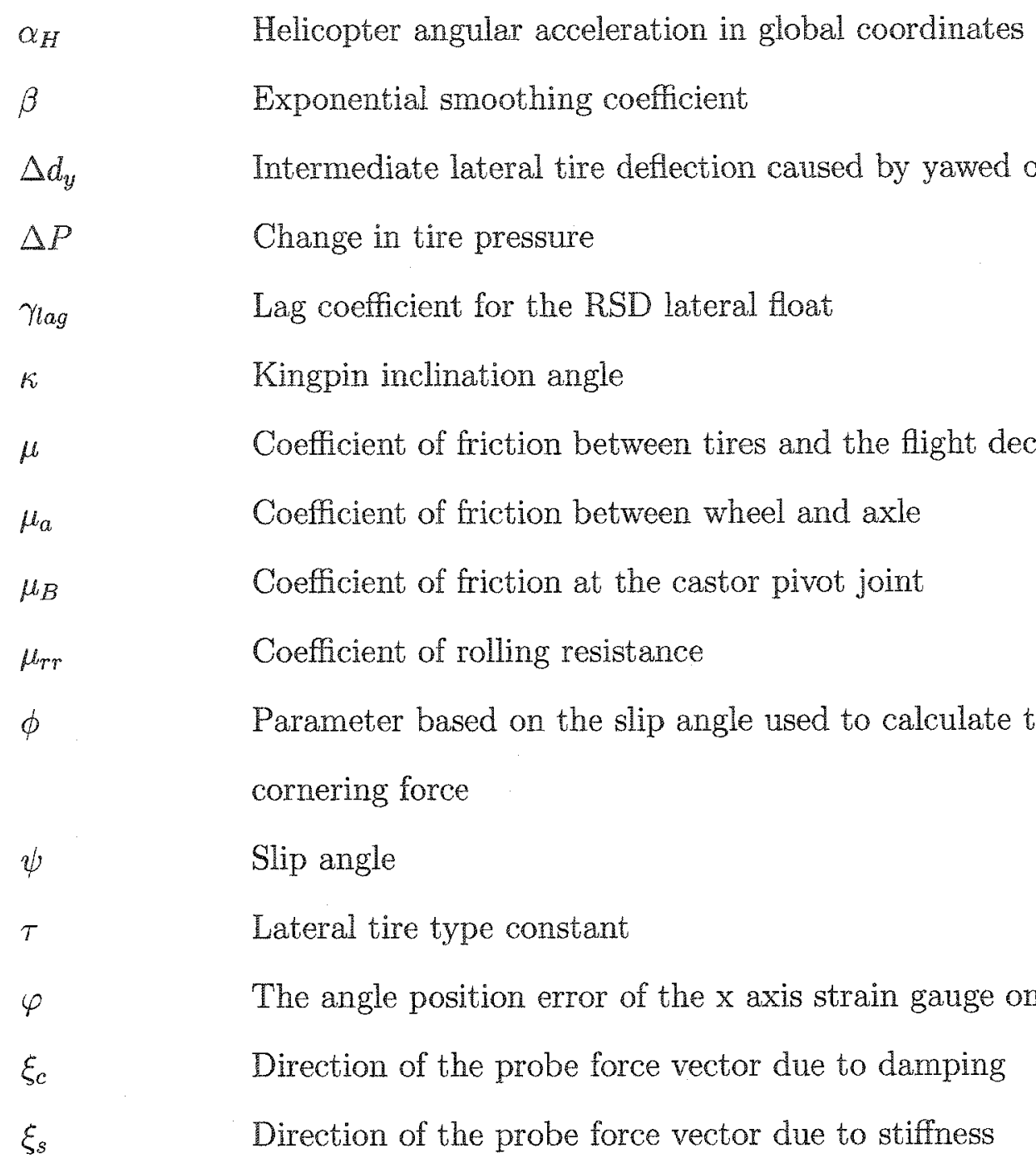




\begin{tabular}{|c|c|}
\hline$F^{a c t}$ & The actual force vector acting on the probe \\
\hline$F_{B}$ & Force vector acting on the joint pivot point $\mathbb{B}$ \\
\hline$F_{c}$ & Probe force vector due to the probe damping \\
\hline$F_{c t_{1}}$ & Force vector acting on the first (left) castor tire \\
\hline$F_{c t_{2}}$ & Force vector acting on the second (right) castor tire \\
\hline$F_{d x}$ & Aerodynamic drag force vector acting in the belicopter longitudinal direction \\
\hline$F_{d y}$ & Aerodynamic drag force vector acting in the helicopter lateral direction \\
\hline$F_{k}$ & Vector containing known forces and moments \\
\hline$F^{m e a}$ & The measured force vector acting on the probe \\
\hline$F_{m l t_{1}}$ & Force vector acting on the first (left) main left tire \\
\hline$F_{m l t_{2}}$ & Force vector acting on the second (right) main left tire \\
\hline$F_{m r t_{1}}$ & Force vector acting on the first (left) main right tire \\
\hline$F_{m r t_{2}}$ & Force vector acting on the second (right) main right tire \\
\hline$F_{P}$ & Force vector acting on the probe \\
\hline$F_{s}$ & Probe force vector due to the probe stiffness \\
\hline$F_{t}$ & Vector of tire forces \\
\hline$F_{u}$ & Vector containing unknown forces and moments \\
\hline$K_{t}$ & Vector of tire stiffnesses \\
\hline$q$ & State vector \\
\hline$R_{B / C}$ & $\begin{array}{l}\text { Position vector of castor pivot point with respect to the castor centre } \\
\text { of mass }\end{array}$ \\
\hline$R_{B / H}$ & $\begin{array}{l}\text { Position vector of castor pivot point with respect to the helicopter centre } \\
\text { of mass }\end{array}$ \\
\hline$R_{C}$ & Position vector of the castor assembly \\
\hline$R_{H}$ & Position vector of the helicopter body \\
\hline$R_{c t / C_{1}}$ & Position vector of the first (left) castor tire with respect to the castor \\
\hline
\end{tabular}


centre of mass

$R_{c t / C_{2}} \quad$ Position vector of the second (right) castor tire with respect to the castor centre of mass

$R_{C P x / H} \quad$ Position vector of the $\mathrm{x}$ centre of pressure with respect to the helicopter centre of mass

$R_{C P y / H} \quad$ Position vector of the y centre of pressure with respect to the helicopter centre of mass

$R_{m l t / H_{1}} \quad$ Position vector of the first (left) main left tire with respect to the helicopter centre of mass

$R_{m l t / H_{2}} \quad$ Position vector of the second (right) main left tire with respect to the helicopter centre of mass

$R_{m r t / H_{1}} \quad$ Position vector of the first (left) main right tire with respect to the helicopter centre of mass

$R_{m r t / H_{2}} \quad$ Position vector of the second (right) main right tire with respect to the helicopter centre of mass

$R_{P} \quad$ Position vector of probe in global coordinates

$R_{P / H} \quad$ Position vector of probe with respect to the helicopter centre of mass

$R_{R S D} \quad$ Position vector of $\mathrm{RSD}$ in global coordinates

$R_{R S D / P} \quad$ Probe deflection

$R_{t} \quad$ Displacement of wheel hub

$V_{C} \quad$ Velocity vector of castor assembly

$V_{H} \quad$ Velocity vector of the helicopter body

$V_{t} \quad$ Velocity of wheel hub

$V_{w} \quad$ Velocity vector of wind acting on the aircraft

$v_{R S D} \quad$ Vector of RSD velocities

$x \quad$ Gauss elimination demonstration vector 
$\Delta r_{t}$

Change in position of wheel hub

\section{Matrices:}

$a$

$B$

D

$M$

T
Gauss elimination demonstration matrix

Kinematic matrix

Matrix used to isolate the unknown forces

Mass matrix

Transformation Matrix 


\section{Contents}

Acceptance $\quad$ ii

Abstract $\quad$ iii

Acknowledgements iv

List of Symbols vi

Contents xiii

List of Figures xvi

List of Tables $\quad$ xxi

1 Introduction 1

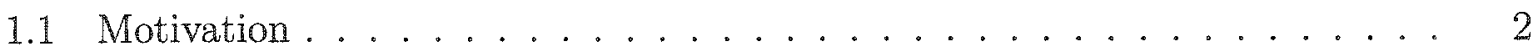

1.2 Helicopter Securing and Handling Systems . . . . . . . . . . . 6

1.2.1 Active Securing . . . . . . . . . . . . . . 7

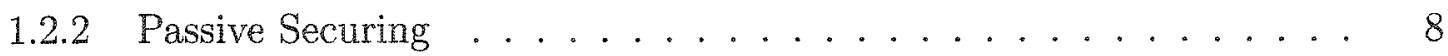

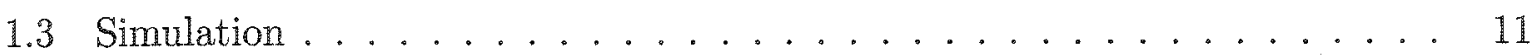

1.3.1 Helicopter Securing . . . . . . . . . . . . . 12

1.3 .2 Tire Models . . . . . . . . . . . . . . . . 14 
1.3.3 Airplane Taxiing . . . . . . . . . . . . . . 16

1.4 Objectives . . . . . . . . . . . . . . . 17

1.5 Thesis Overview. . . . . . . . . . . . . . . . . . 18

2. Mathematical Model 20

2.1 Helicopter Dynamics Model . . . . . . . . . . . . . . . . . . . . 21

2.1.1 Equations of Motion . . . . . . . . . . . . . 22

2.1 .2 Castor Joint Model . . . . . . . . . . . . . . . . . . . . 30

2.1 .3 Tire Model . . . . . . . . . . . . . . . . . . . . . . 36

2.1.4 RSD/Probe Model . . . . . . . . . . . . . . 47

2.1 .5 Aerodynamic Model . . . . . . . . . . . . . . . 50

2.2 Control Model . . . . . . . . . . . . . . . . . . . . . . 51

2.2 .1 Joystick Input . . . . . . . . . . . . . . . . . . 51

2.2 .2 File Input $\ldots \ldots \ldots \ldots \ldots \ldots \ldots \ldots \ldots$

2.2 .3 Controller Input . . . . . . . . . . . . . . 56

2.3 Simulation Model . . . . . . . . . . . . . . . . . . . 56

2.3 .1 Numerical Methods . . . . . . . . . . . . . . . . . . 58

2.3 .2 Transformation Matrices . . . . . . . . . . . . . . 60

3 Verification and Validation 61

3.1 Verification . . . . . . . . . . . . . . . . . 61

3.1.1 Equations of Motion Comparison . . . . . . . . . . . 62

3.1.2 Castor Joint Model Comparison . . . . . . . . . . . . . 64

3.1 .3 Tire Model Comparison $\ldots \ldots \ldots \ldots \ldots$

3.1.4 RSD/probe Model Comparison . . . . . . . . . . 67

3.1.5 Aerodynamic Model Comparison ... . . . . . . . . . . . . 69

3.1.6 Joystick Control Model Comparison . . . . . . . . . . . . . . . . 69 


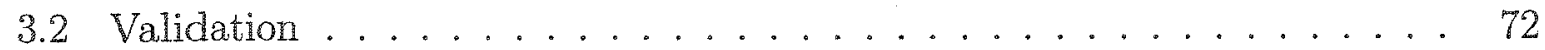

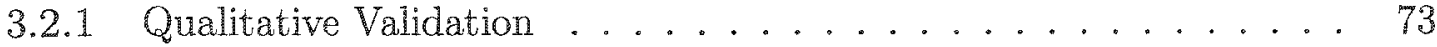

3.2 .2 Partial Tire Validation . . . . . . . . . . . . 74

3.2.3 Validation Parameters ................ 77

3.2 .4 Validation Results .................. 87

4 Discussion and Conclusion $\quad 125$

$\begin{array}{ll}\text { References } & 129\end{array}$

$\begin{array}{ll}\text { Appendices } & 136\end{array}$

A Heliman Users Guide $\quad 137$

B Analytical Solution $\quad 149$ 


\section{List of Figures}

1.1 Different elements of the helicopter/ship dynamic interface . . . . . . 3

1.2 Active and passive securing principles and deck lock/grid . . . . . . 8

1.3 Positional feedback: constant tension cable . . . . . . . . . . . 10

1.4 ASIST system . . . . . . . . . . . . . . . . 11

1.5 Identification of forces on a secured helicopter . . . . . . . . . 13

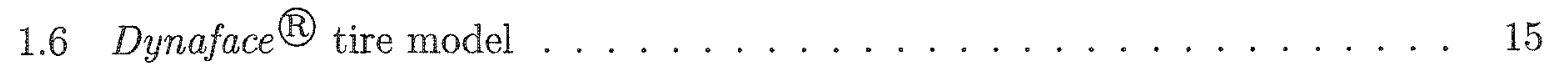

2.1 Schematic of the dynamic helicopter model . . . . . . . . . . 21

2.2 Free-body diagram of the main helicopter body and castorable wheel assembly. 26

2.3 Illustration of the Coulomb friction and viscous damping torques acting on

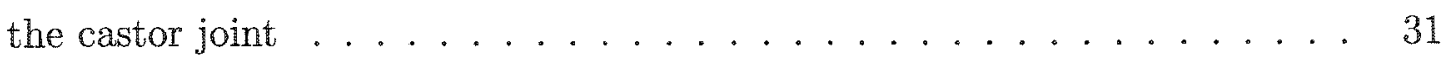

2.4 Standard and modified Coulomb friction models . . . . . . . . . . . 32

2.5 Kingpin inclination angle . . . . . . . . . . . . . 33

2.6 Rotated castor wheel demonstrating the effect of the kingpin inclination angle 34

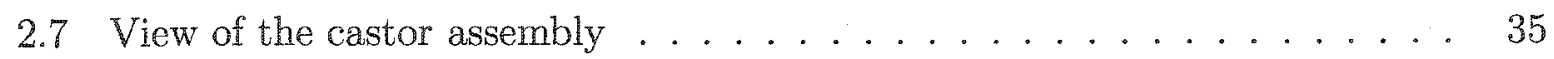

2.8 Flowchart of the implemented tire model . . . . . . . . . . . 37

2.9 Vertical tire deflection ....................... 40

2.10 Lateral tire deflection . . . . . . . . . . . . . . . . 41

2.11 The slip defined in the wheels frame of reference . . . . . . . . . 42

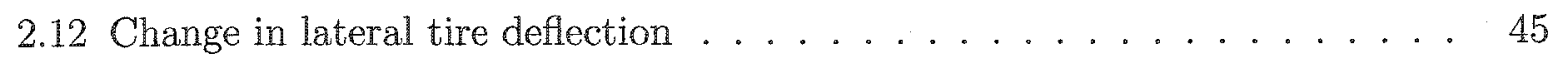


2.13 Schematic of RSD/probe model showing the spring/damper representation

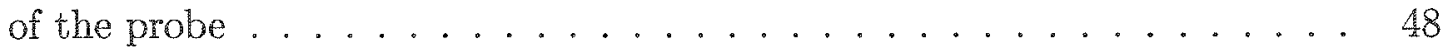

2.14 Stiffness ellipse for the large deflection stiffness . . . . . . . . . . . 49

2.15 Graph showing how the joystick specifies the RSD velocity . . . . . 52

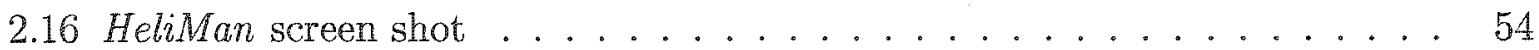

2.17 Labelled HeliMan screen shot ................. 55

2.18 Flow chart of simulation . . . . . . . . . . . . . 57

3.1 Demonstration of the relaxation of tire deflections in the main tires $\ldots . .67$

3.2 RSD positions within the box corresponding to the different situations . . . 70

3.3 ITI's dead load test vehicle (DLTV) . . . . . . . . . . 72

3.4 Vertical tire deflection versus vertical tire force for the main tire . . . . . 75

3.5 Vertical tire deflection versus vertical tire force for the tail tire . . . . . 75

3.6 Lateral tire stiffness versus vertical tire force for the main tire . . . . . . 76

3.7 Lateral tire stiffness versus vertical tire force for the tail tire . . . . . 76

3.8 Comparison of manoeuvre results using different time steps . . . . . . . 79

3.9 Initial position of helicopter and the direction RSD is moved in HeliMan to test the effect of changing the mass moment of inertia . . . . . . . . 80

3.10 The effect of lowering the mass moment of inertia in HeliMan . . . . . . . 81

3.11 Initial position of DLTV and direction RSD is moved for the rolling resis-

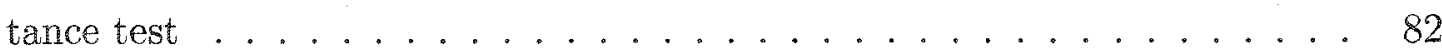

3.12 Component forces acting on the main probe for the rolling resistance test . 84

$3.13 \mathrm{X}$ component forces acting on the main probe during the straight track

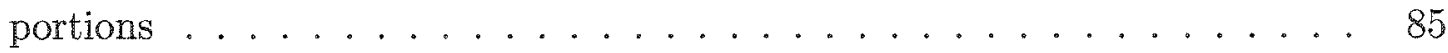

$3.14 \mathrm{X}$ component forces acting on the main probe during the first traversing stage 86

3.15 Initial position of DLTV and direction RSD is moved for Case $1 \ldots . .87$

3.16 Comparison of the RSD claw y-position for Case $1 \ldots \ldots 8$ 
3.17 Comparison of the force acting on the main probe in the DLTV's $y$-direction

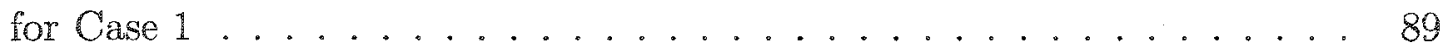

3.18 Comparison of the force acting on the main probe in the DLTV's $\mathrm{x}$-direction

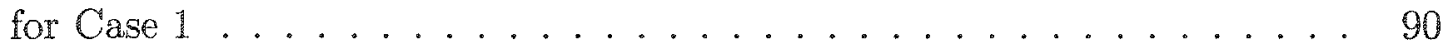

3.19 Comparison of the lateral tire deflections for Case 1 . . . . . . . . . 91

3.20 Demonstration of the simulations tendency to balance out the lateral tire

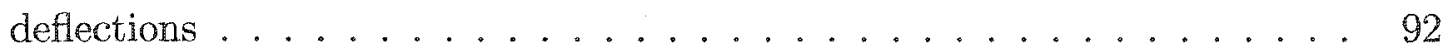

3.21 Schematic of forces acting on the tire axle ........... 93

3.22 Comparison of the force acting on the probe in the DLTV's $\mathrm{x}$-direction for Case 1 with the new axle friction component . . . . . . . . . . . 94

3.23 Initial position of DLTV and direction RSD is moved for Case 2 . . . . . 94

3.24 Comparison of the RSD claw x-position for Case $2 \ldots \ldots 5$

3.25 Comparison of the RSD claw y-position for Case $2 \ldots \ldots$. . . . . . 96

3.26 Comparison of the force acting on the probe in the DLTV's $\mathrm{x}$-direction for

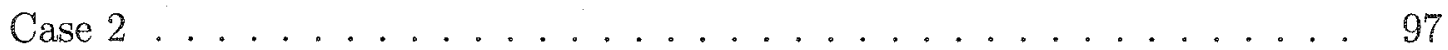

3.27 Comparison of the force acting on the probe in the DLTV's y-direction for Case $2 \ldots \ldots \ldots \ldots \ldots \ldots$

3.28 Schematic of strain gauge placement on the probe . . . . . . . . . 99

3.29 Comparison of the lateral tire deflections for Case $2 \ldots \ldots$. . . . . . 100

3.30 Initial position of DLTV and direction RSD is moved for Case $3 \ldots \ldots 1$

3.31 Comparison of the RSD claw x-position for Case $3 \ldots \ldots . . \ldots 102$

3.32 Comparison of the RSD claw y-position for Case $3 \ldots \ldots 103$

3.33 Comparison of DLTV orientation for Case $3 \ldots \ldots \ldots$

3.34 Comparison of castor orientation for Case $3 \ldots \ldots$. . . . . . . . 104

3.35 Comparison of the force acting on the main probe in the DLTV's $\mathrm{x}$-direction for Case $3 \ldots \ldots \ldots \ldots \ldots . \ldots \ldots$ 
3.36 Comparison of the force acting on the main probe in the DLTV's y-direction for Case $3 \ldots \ldots \ldots \ldots \ldots . \ldots \ldots$

3.37 Comparison of the lateral tire deflections for Case $3 \ldots \ldots$. . . . . 106

3.38 Comparison of simulated $\mathrm{x}$ component of the main probe forces resulting from Case 3 for two different mass moments of inertia . . . . . . . . 107

3.39 Initial position of DLTV and direction RSD is moved for Case 4 . . . . 108

3.40 Comparison of the RSD claw $\mathrm{x}$-position for Case $4 \ldots \ldots 110$

3.41 Comparison of the RSD claw y-position for Case $4 \ldots \ldots$. . . . . . . 110

3.42 Comparison of castor orientation for Case $4 \ldots \ldots \ldots$. . . . . . 111

3.43 Comparison of DLTV orientation for Case $4 \ldots \ldots \ldots$. . . . . . 111

3.44 Comparison of the force acting on the main probe in the DLTV's $\mathrm{x}$-direction for Case $4 \ldots \ldots \ldots \ldots \ldots \ldots$. . . . . . . . . . . . . . . . . . . . . . . .

3.45 Comparison of the force acting on the main probe in the DLTV's y-direction for Case $4 \ldots \ldots \ldots \ldots \ldots \ldots$

3.46 Comparison of the lateral tire deflections for Case $4 \ldots \ldots 113$

3.47 Initial position of DLTV and direction RSD is moved for Case $5 \ldots \ldots 114$

3.48 Comparison of the RSD claw x-position for Case $5 \ldots \ldots \ldots$

3.49 Comparison of the RSD claw y-position for Case $5 \ldots \ldots \ldots$

3.50 Comparison of DLTV orientation for Case $5 \ldots \ldots \ldots 116$

3.51 Comparison of castor orientation for Case $5 \ldots \ldots \ldots 117$

3.52 Comparison of the force acting on the main probe in the DLTV's $\mathrm{x}$-direction for Case $5 \ldots \ldots \ldots \ldots \ldots \ldots \ldots$

3.53 Comparison of the force acting on the main probe in the DLTV's $y$-direction for Case $5 \ldots \ldots \ldots \ldots \ldots \ldots$

3.54 Comparison of the lateral tire deflections for Case $5 \ldots \ldots 119$

3.55 Initial position of DLTV and direction RSD is moved for Case 6 . . . . 120 
3.56 Comparison of the RSD claw x-position for Case $6 \ldots \ldots \ldots$

3.57 Comparison of the RSD claw y-position for Case $6 \ldots \ldots \ldots$

3.58 Comparison of the force acting on the main probe in the DLTV's $\mathrm{x}$-direction for Case $6 \ldots \ldots \ldots \ldots \ldots \ldots$

3.59 Comparison of the force acting on the main probe in the DLTV's y-direction for Case $6 \ldots \ldots \ldots \ldots \ldots \ldots$

3.60 Comparison of the lateral tire deflections for Case $6 \ldots \ldots$. . . . 124 


\section{List of Tables}

3.1 Verification results for the equations of motion $\ldots \ldots \ldots 63$

3.2 Verification results for the castor joint model . . . . . . . . . 64

3.3 Verification results for the tire force model . . . . . . . . . . . 66

3.4 Verification results for the tire deflection model $\ldots \ldots \ldots 6$

3.5 Verification results for the RSD/probe model . . . . . . . . . 68

3.6 Verification results for the aerodynamic model . . . . . . . . . . . . . 69

3.7 Verification results for the joystick control model . . . . . . . . . . . 71 


\section{Chapter 1}

\section{Introduction}

Over the past fifty years, helicopters have changed the way the world's navies and coastguards are built. The helicopter's ability to land on a small flight deck has expanded the capabilities of smaller ships, by increasing their areas of influence. This has given smaller countries the ability to field low-cost, effective navies.

According to Hunt [1], the first helicopter was operated successfully on the deck of a ship in the mid 1920's by Wing Commander Brie of England on the Italian Navy destroyer Fiume. It was not until the 1950's that the Canadian Navy decided to perform its own study on operating a helicopter on a small ship [2]. In September 1956 LCdr Rod Ray and LCdr John Laurie conducted the first helicopter platform trials in Canada on the Prestonian class frigate, HMCS Buckingham. Once the concept was proven, people started to develop methods to make operating helicopters on ships safer and easier.

Concerns about securing the helicopter once it landed was a top priority among the countries developing this capability. This was especially true for navies operating in the rough North Atlantic. The first securing system was developed by the British Royal Navy and consisted of a helicopter crewman firing a specially designed harpoon into a six foot square steel grid on the flight deck upon landing [2]. The Canadian Navy was 
convinced that a constant tension cable system was needed to help guide the helicopter during hovering and landing [2]. The harpoon system did not provide this feedback to the pilot. The Canadian Navy currently uses a constant tension cable system for their Sea King helicopters.

It was also found that a hangar was needed to protect the helicopter from severe weather conditions and make it safer to perform any maintenance on the helicopter. To do this the helicopter needed to be straightened and then traversed from the flight deck and into the hangar. With the space on a ship being so valuable the hangar/helicopter clearances are very small. This makes the manoeuvring of a helicopter into the hangar a very delicate operation.

This process was originally done with a series of traversing and guiding winches. In some cases the helicopter had to be unsecured from one system and hooked up to another for traversing. As securing devices have become more modern, the trend has been to integrate the manoeuvring capabilities with the securing capabilities. A detailed review of these helicopter securing and handling technologies is carried out in Section 1.2.

This thesis will attempt to help improve on-deck helicopter operations by simulating the planar manoeuvring of a helicopter. This chapter highlights the results of an extensive literature review of topics that will be used to produce and validate the transient handling simulation.

\subsection{Motivation}

As the capability of computers has increased, simulation has become a more integral part of the design process. The biggest advantage to modelling and simulating a system is that it allows the engineer to predict the behaviour of the system before it is built. A simulation can also be used to analyze an existing system to improve a design or predict what might 
happen during different or unusual operating conditions.

Simulation has been extended to real-time simulators that have revolutionized training methods. According to Woods and Lawrence [3] flight-simulator-based training costs 1/10th that of training in a real airplane and offers no safety risks to the student, instructor and the general public. Simulators have also been constructed to train tank, ship, and helicopter crews.

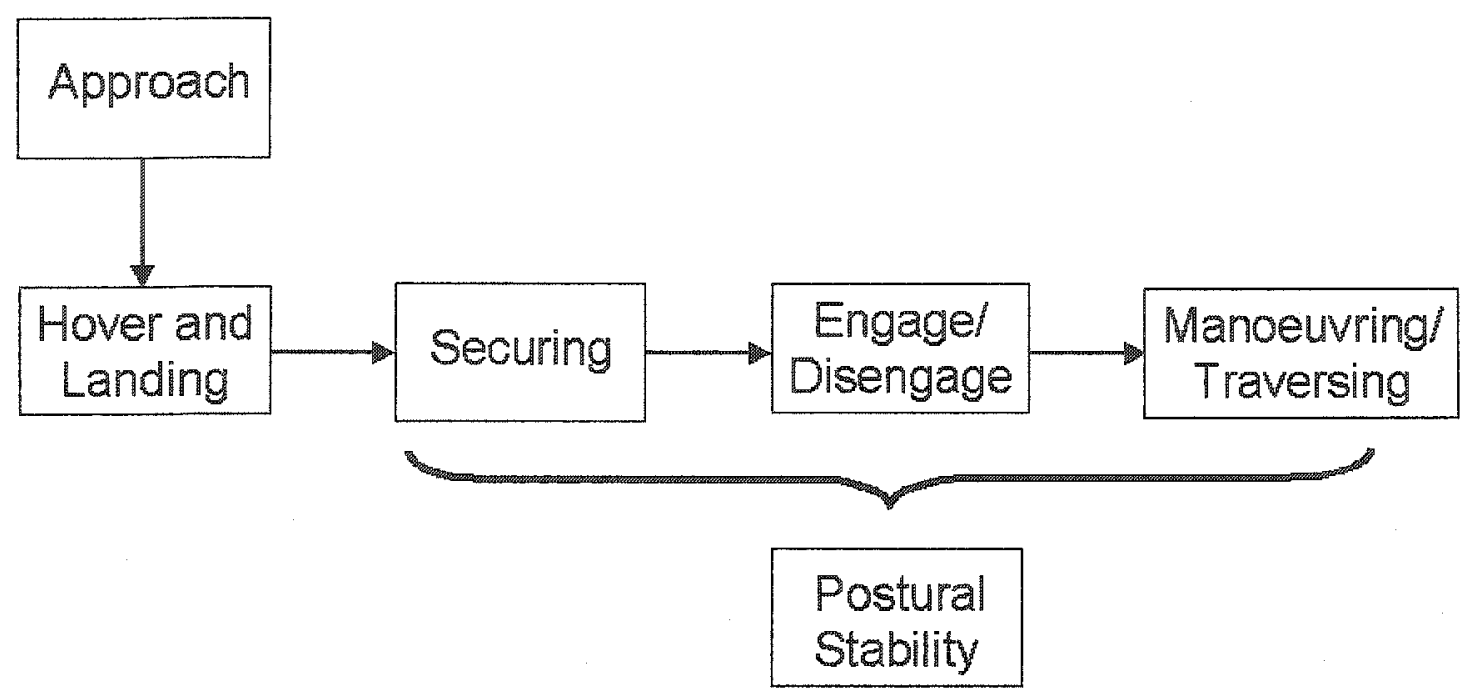

Figure 1.1: Different elements of the helicopter/ship dynamic interface

For the past fifteen years, Indal Technologies Inc. (ITI), a developer of shipboard helicopter recovery and on-deck handling systems, has been developing a helicopter/ship dynamic interface simulation called Dynaface ${ }^{\circledR}[4]$ to support the design of their three systems: Aircraft Ship Integrated Secure and Traverse (ASIST); Twin-Claw ASIST (TCASIST); and Recovery Assist, Secure and Traverse (RAST). Langlois and Tadros [5] define 'dynamic interface analysis' as a comprehensive term referring to the investigation of all aspects relating to the effect of ship motion on embarked helicopter operations. Figure 1.1 illustrates the different elements involved in 'dynamic interface analysis'. This includes the approach, hover and landing, on-deck securing, rotor engage and disengage, and handling phases. The engage and disengage phase occurs very quickly and is discussed as part of 
the securing phase. The postural stability of the deck crew must also be considered when performing any tasks required in the dynamic interface. The securing phase is reviewed in greater detail in Sections 1.2 and 1.3.

Colwell [6] breaks down the approach, hover and landing phases for the Canadian Navy using ITI's RAST system as follows:

- the helicopter approaches the ship from astern on the ship's port side;

- the helicopter hovers laterally across the ship to the starboard side of the flight deck;

- the hauldown cable is connected from the ship to the helicopter;

- the pilot takes a "high hover" position over the RSD, where

- tension is applied to the hauldown cable, and

- the pilot waits for a "quiescent period";

- the pilot initiates descent to "low hover";

- the pilot requests clearance to land from the Landing Safety Officer (LSO); and,

- the LSO will usually either;

- grant clearance to land;

- advise the pilot to re-position to keep centred over the RSD;

- advise the pilot to wait for ship motions to reduce; or,

- call a "wave off", after which the pilot returns to high hover.

Even with modern securing equipment, the pilot still has to wait for a quiescent period. Quiescent periods are short lulls in the ship motion that occur less frequently as the sea conditions get rougher, but do occur no matter how rough the conditions get. Determining when a quiescent period is about to occur is very difficult and requires the experience of a seasoned LSO. Ferrier et al $[7,8]$ have attempted to create a monitoring system called the Landing Period Designator (LPD) that can identify these quiescent periods.

Carignan et al [9] have developed a simulation method to evaluate the handling qualities of a helicopter in high and low hover phases. This is done on dry land with a modified 
Bell 205 helicopter and a motorized position cueing apparatus. The pilot attempts to visually line up two points on the apparatus that move in the horizontal and vertical axes. The ship motions are simulated through this apparatus. The helicopter is instrumented to record all the relevant data needed.

The hover phase has also been addressed aerodynamically by Zan [10] and Lee [11]. They examined the effect that the airwake has on the rotor thrust and fuselage loads of a Sea King while hovering over the flight deck of a Canadian Patrol Frigate. McKillip et al [12], Lee et al [13], Aponso and Jewell [6], and Keller and Smith [14] have also considered the effects that the ship's airwake has on an approaching and hovering helicopter. Colwell [15] studied the effects of a ship on a hovering helicopter using fourier analysis and flight test data. Bradley and Tumer [16] have made an attempt to simulate the pilot hovering and landing a helicopter on the flight deck of a ship.

The postural stability aspect of the dynamic interface refers to the crew's ability to perform manual tasks on the flight deck, such as attaching the constant-tension cable, in rough sea conditions. This area is studied for much broader applications than just helicopter securing, from fishermen pulling in their catch to rescuing an overboard shipmate, rough sea conditions can compromise an experienced crew's effectiveness. O'Hanlan and McCauley [17] have correlated motion sickness incidences with vertical acceleration. Schoenberger [18] created a subjective magnitude scale to estimate how dangerous it would be to perform tasks in certain conditions. The next step was taken by Graham et al [19] when they analyzed a human proportioned block on a ship and calculated when it tipped or slid and called it a Motion Induced Interruption (MII). An MII is defined as a predicted point in time when a sailor would have to stop what they were doing to keep their balance. Wedge and Langlois [20] found that this model was conservative and thus added a multi-body aspect to the model along with force feedback control to some of the joints. This has proven successful in creating a more accurate model. It is also important to 
note, as discussed by Lloyd [21], that the size and design of the ship can greatly influence how it will react in rough sea conditions. A sailer performing a task on an aircraft carrier in certain conditions may have trouble doing the same task on a frigate in similar sea conditions.

The element that has not been addressed is the on-deck handling phase of the operation, where the aircraft is straightened and traversed along the deck and into the ship's hangar. The current method for analyzing the handling element is to run Dynaface ${ }^{\circledR}$ simulations at static points along a predicted path on the flight deck. This research project, sponsored jointly by ITI, Materials and Manufacturing Ontario (MMO), and Carleton University (CU), will address the on-deck handling of the helicopter.

The purpose of this thesis is to develop and validate a transient helicopter handling simulation, which can be initially used on its own and subsequently interfaced with $D y$ naface ${ }^{\circledR}$. This simulation will provide the first steps towards a more economical training method and help in the design of future handling systems, which includes the possibility of an automated manoeuvring system. To do this, the simulation must be flexible enough to model a variety of helicopter configurations. The importance of having flexible simulation capabilities is demonstrated by Langlois et al [22] in their geometric and inertial parameter study done for a helicopter's securing requirements. For the purpose of this project, significant differences in configuration include nose versus tail wheel configuration, the number of tires per suspension station, and the possibility of steerable versus castorable nose/tail wheels.

\subsection{Helicopter Securing and Handling Systems}

Over the past fifty years, ship flight deck helicopter securing has evolved significantly. In the beginning, helicopters where lashed down with chains. This was extremely dan- 
gerous for the crew who had to manually secure the helicopter, making the conditions in which the helicopter could be landed successfully and safely very limited. To limit the crew's involvement in the securing process, automatic securing methods were subsequently developed.

To traverse the helicopter into the hangar was also very dangerous. The deck crew had to manually move the helicopter while lashing and unlashing the helicopter down while traversing along the path. As with securing, traversing the helicopter was limited to very low sea conditions prior to the introduction of integrated helicopter securing and handling systems. These important developments have reduced the required number of on-deck personnel required and in doing so expanded the operational envelope in which traversing can be performed safely.

Demands for the ability to secure and traverse helicopters in rougher sea conditions have fuelled the creation of systems that limit the crew's physical involvement as much as possible. With the need to carry out helicopter operations in the North Atlantic, Canada has played a leading role in developing the ability to secure and traverse helicopters in rough seas. Most helicopter securing systems can be broken down into two types: active securing with separate traversing systems; and passive securing with integrated traversing capabilities citehel01.

\subsubsection{Active Securing}

An active securing system creates a constant downward force on the helicopter using a mechanical/hydraulic system onboard the helicopter. The system transmits only axial loads, which it relies on to increase lateral securing forces between the tires and deck. The lower diagram in Figure 1.2A demonstrates an active securing system. If, as in Figure 1.2A the device is allowed to pivot at the aircraft attachment point, a fraction of the axial force is transmitted laterally. Figure $1.2 \mathrm{~B}$ is an example of an active securing system. 


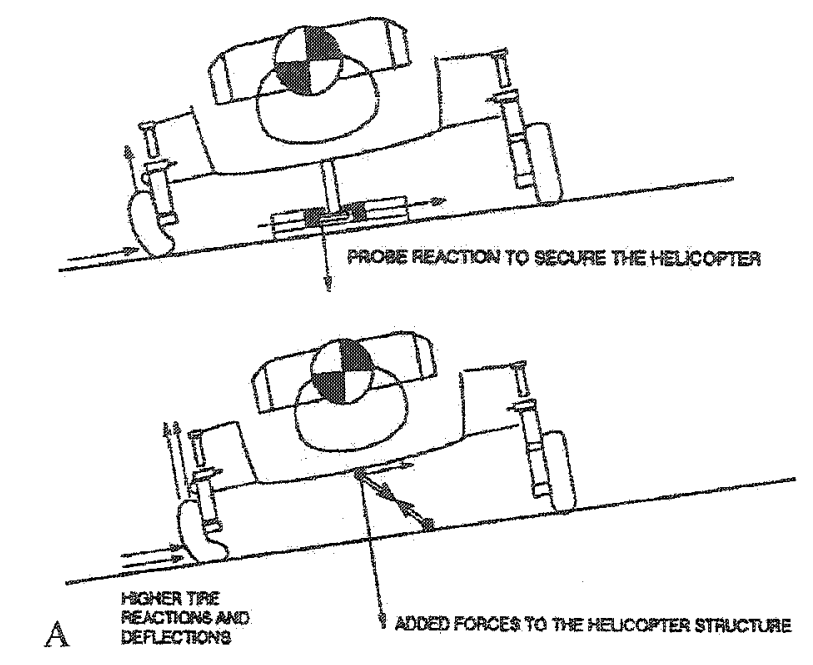

Figure 1.2: A. Securing principles: passive (upper) and active (lower) [23] and B.

Deck lock/grid [24]

DCN International is the largest manufacturer of active securing systems with their harpoon and grid landing system. A long harpoon-type device is attached to the helicopter and when landing, must be inserted into a hole in the grid on the flight deck. DCN also makes a separate system called Samahé for handling the helicopter on the flight deck. The Samahé attaches to a point on the helicopter and traverses along a track on the flight deck.

\subsubsection{Passive Securing}

A passive securing system fixes the helicopter in place without applying a downward force. A rigid probe attached to the helicopter is restricted from moving in the axial, lateral and longitudinal directions by the supporting structure on the deck. The upper diagram in Figure 1.2A demonstrates a passive securing system. Reaction forces are generated between the probe and supporting structure caused by the relative motions between the helicopter and ship. These reaction forces are limited only by the strength of the probe and the supporting structure. Unlike the active system, a passive system supports the helicopter in both the axial and lateral directions. It is important to note that the tires 
still share the lateral load with the device, but not to the same extent as the active system.

ITI uses the passive securing method in their RAST, ASIST and TC-ASIST securing systems. All three ITI systems are connected to tracks that give them traversing capabilities without having to transfer the helicopter to a separate traversing system.

\section{RAST}

RAST (Recovery Assist, Secure and Traverse) uses a single Rapid Securing Device (RSD) to secure and traverse the helicopter on the flight deck. A probe, attached to the helicopter fuselage, projects from the underside of the helicopter, which provides the securing point. The general RAST landing procedure is described in Section 1.1. The process of touching down will be described in greater detail here.

During a quiescent period, the pilot requests the LSO to apply tension to the recovery assist cable. This tension directs the helicopter toward the designated landing area, as the pilot slowly flies the aircraft onto the deck. Figure 1.3 illustrates how the constant tension cable works as a feedback mechanism. Immediately upon touchdown, the LSO increases the cable tension and closes the RSD's arresting beams thereby passively securing the helicopter. Straightening the helicopter is a nontrivial task with a RAST RSD as it is only capable of applying longitudinal motions to the helicopter. The helicopter must be straightened with motorized winches and cables attached to the fuselage that apply lateral loads in combination with the longitudinal motion of the helicopter.

\section{ASIST}

As with the RAST system, ASIST (Aircraft Ship Integrated Secure and Traverse), uses a single RSD to keep the helicopter secure when it is on the flight deck or in the hangar. Instead of a tensioned cable to provide position cues during recovery, ASIST incorporates an electro-optical tracking system, which controls the RSD to follow the aircraft fore 


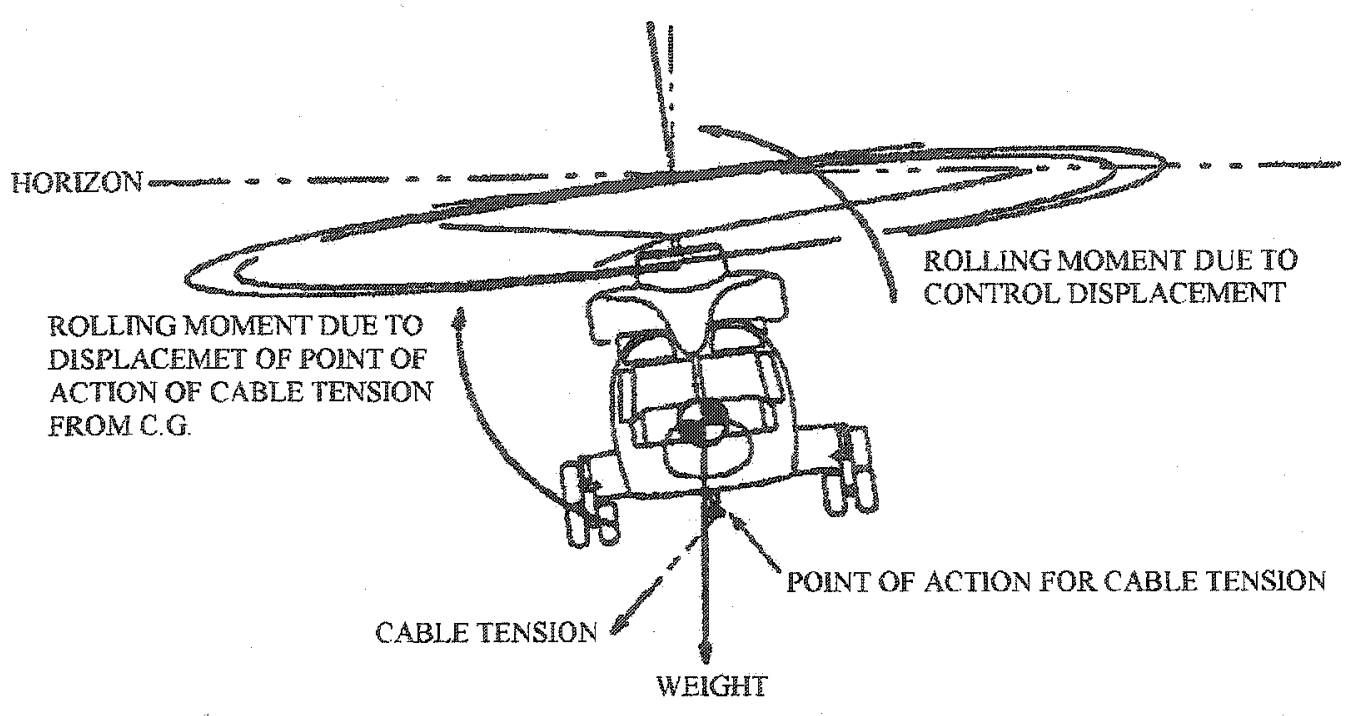

Figure 1.3: Positional feedback: constant tension cable [24]

and aft during hover and landing [25]. Light signals are used to help the pilot keep the helicopter positioned over the landing spot during a period of quiescence. The RSD can automatically secure the probe upon touchdown within 2 seconds. Alternatively, as with RAST, securing can be initiated manually. Figure 1.4 illustrates the ASIST system.

ASIST has superior manoeuvring capabilities by offering lateral control of the RSD claw. This means the entire straightening and traversing procedure can be accomplished by a single operator, without the need for motorized winches and cables.

\section{TC-ASIST}

TC-ASIST (Twin-Claw Aircraft Ship Integrated Secure and Traverse) is a larger RSD system appropriate for large helicopters that offers superior control with the traversing of the helicopter and does not need a probe attached to the helicopter. The tracking system works the same as the regular ASIST system. Instead of securing the helicopter with a probe, the TC-ASIST RSD secures to the main wheel axles of the helicopter [25]. The TC-ASIST system is currently going through the final design stage at ITI. 


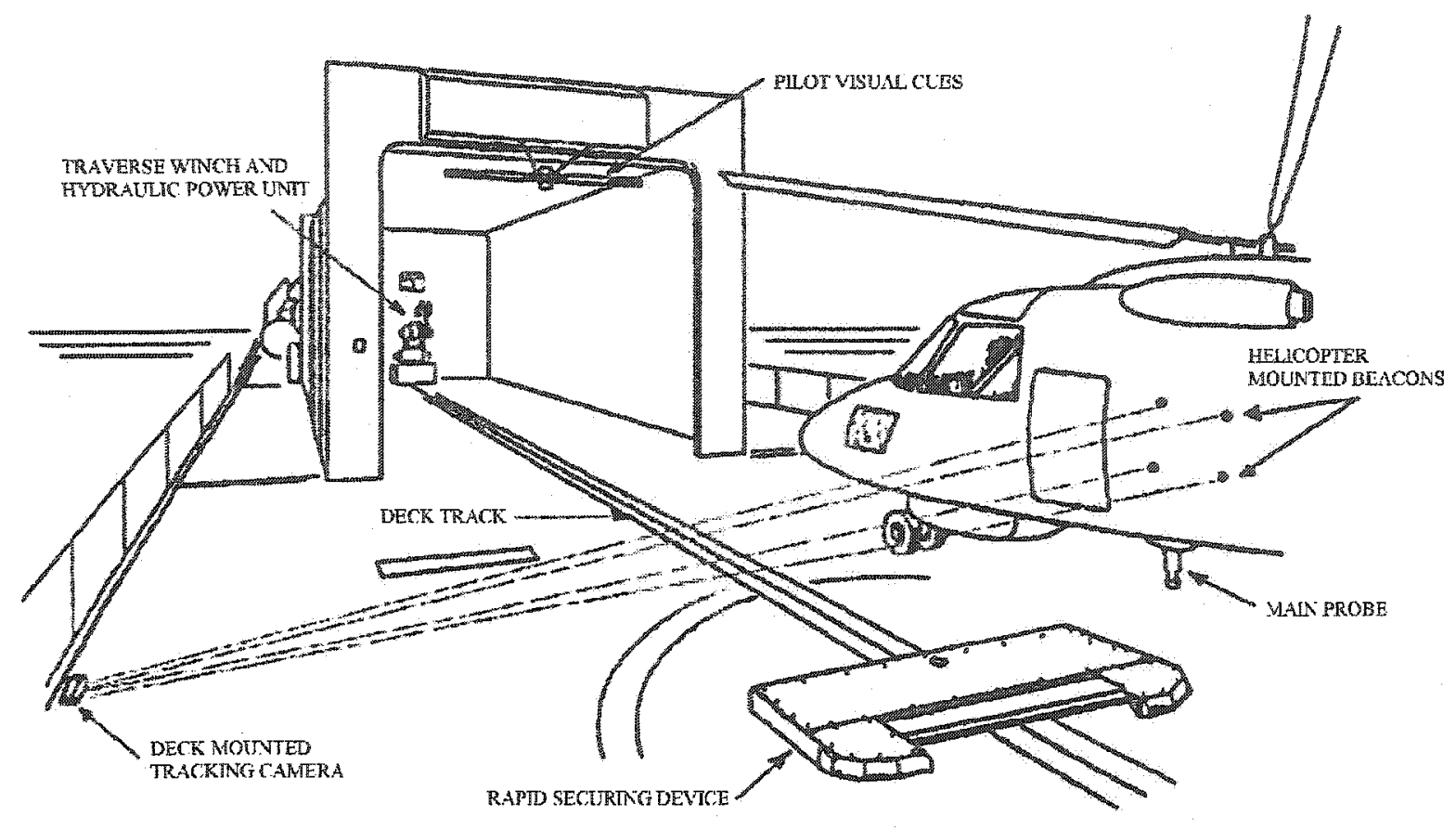

Figure 1.4: ASIST system [26]

\subsection{Simulation}

An investigation of the different on-deck helicopter/ship dynamic interface simulations is presented below. Currently, all simulation efforts have been directed at the securing of the helicopter at a particular location on the ship deck. The securing forces for a traversing helicopter are tested by changing its position on the flight deck and performing a fixed-point securing dynamic analysis. No literature has been found relating to a transient helicopter manoeuvring simulation. This is consistent with the general belief in the dynamic interface community that no such simulation capability currently exists. Consequently, a similar application has been investigated: the taxiing of airplanes. Different tire models are also presented, which will be one of the most important parts of the required transient manoeuvring simulation. 


\subsubsection{Helicopter Securing}

An aircraft secured to a ship flight deck experiences: "loads generated by geometrically nonlinear and time-dependent ship motion, nonlinear and intermittent tire contact and sliding, time and displacement dependent rotor forces, aerodynamic drag forces, and a variety of securing device forces." [4] The historical approaches used for the simulation of this system include static and quasistatic approaches.

The static approach consists of balancing the forces acting on the helicopter while not considering acceleration. The quasistatic approach solves for the helicopter securing forces statically, but considers the helicopter body's acceleration as an inertial force. Wei, Baitis and Meyers [27] and de Smit [28] use the quasistatic approach. Two significant problems exist with static and quasistatic solutions: linearization must be performed; and the equations of motion must be uncoupled.

Attempts have also been made to model the helicopter/ship dynamic interface empirically by running sea trials. Blackwell et al [29] have carried out this type of work for a Sikorsky S-70B-2 Seahawk and FFG-7 frigate using a RAST securing system for the Royal Australian Navy. This experimental approach is accurate, but only for the sea conditions it is being conducted in. The probability of the trials being conducted in severe conditions are low. It is also very expensive to perform sea trials and they are limited to helicopter/ship systems that are already developed.

In 1989, ITI began creating a nonlinear time-domain solution of the coupled equations of motion describing the characteristics of the helicopter/ship system and forces acting on the helicopter. The resulting transient-dynamic simulation software, Dynaface ${ }^{\circledR}$ [4], which is continually being refined replaced quasistatic analysis with an accurate method that considers coupling of parameters and provides event context for simulation events that are of interest. Dynaface $\left.{ }^{(}\right)$can be used with any helicopter configuration being secured with a passive or active securing device in any sea conditions. Dynaface $\left.{ }^{(}\right)$and 
accompanying linear ship motion software SHIPSIM [30] calculate realistic ship motion from response amplitude operators (RAOs) [21] and a specified seaway for combinations of ship heading, ship speed, and sea state. Pure sinusoidal motion and measured ship motion can also be used.

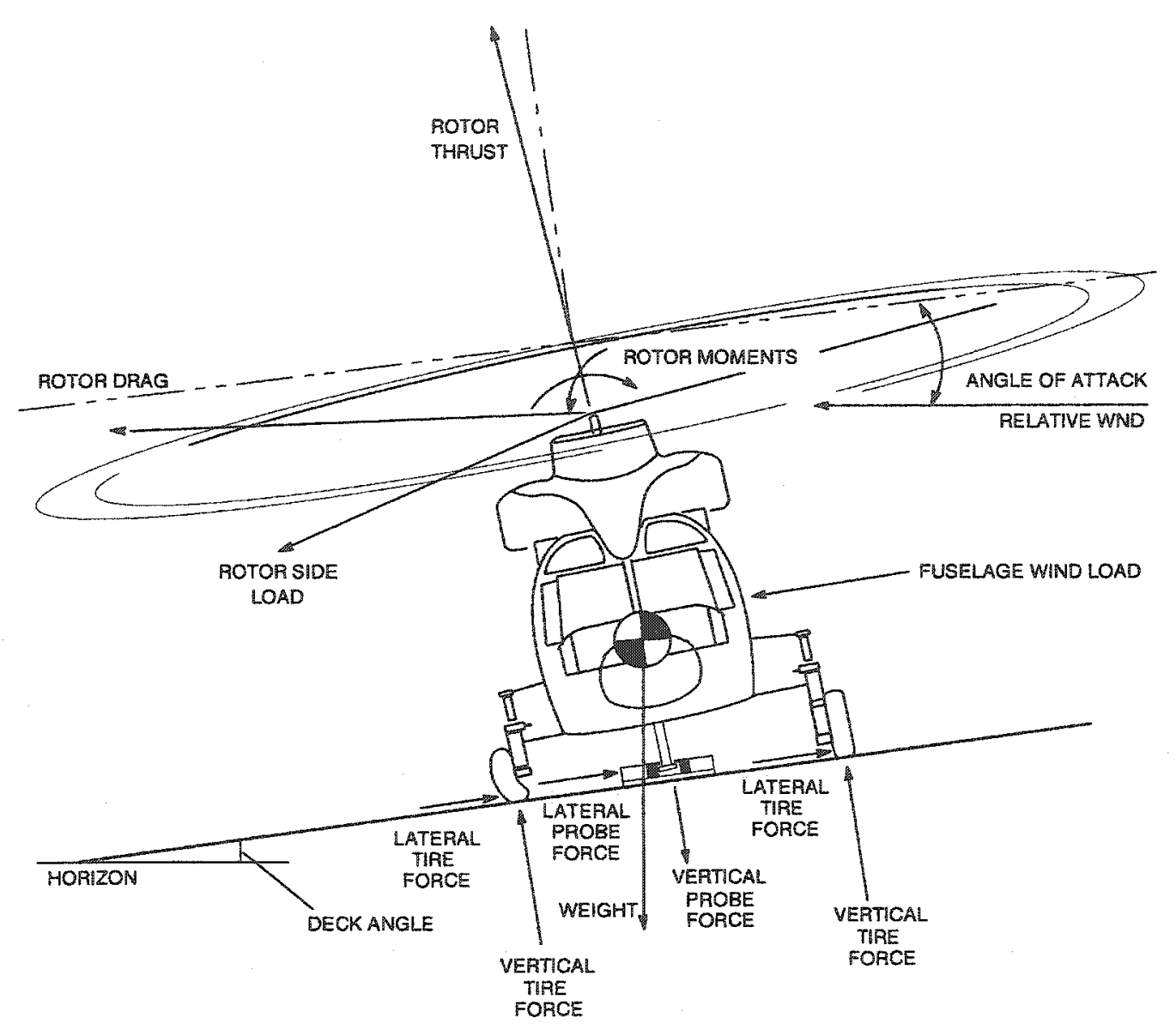

Figure 1.5: Identification of forces on a secured helicopter [25]

Figure 1.5 illustrates some of the output forces that Dynaface ${ }^{\circledR}$ produces. These include the securing forces and the tire reaction forces. Other outputs are also available including: displacement, velocity, and acceleration of the helicopter; tire deflections; suspension deflections; and flags that indicate if a tire has lost contact with the flight deck. An animation data file is also created so the simulation can be played back qualitatively using animation software. 
Over the past few years a team lead by Kang and He $[31,32,33]$ have been developing a helicopter/ship dynamic interface time-domain simulation. They have used a rotorcraft analysis and simulation program called FLIGHTLAB to create their simulation. A large emphasis has been put on the effects of the ship's airwake by using computational fluid dynamics, while the general motion of the ship has been deemphasized by looking it up from a table.

\subsubsection{Tire Models}

Langlois et al [4] describe the tire as one of the most important force-producing elements in the helicopter/ship system as tires form the primary interface between the helicopter and ship. For this reason a large amount of effort was put into trying to find a suitable tire model for the transient manoeuvring simulation. Dynaface ${ }^{(\mathbb{B})}$ is based on an expanded frictional slider tire model outlined in Figure 1.6, but uses an empirical model for longitudinal and lateral tire stiffness developed at NASA by Smiley and Horne [34].

During manoeuvring, the helicopter tires roll at relatively low speed with relatively large deflections compared with conventional vehicle applications. When modelling a slowmoving tire with large deflections, as in this application, accurate methods of modelling relaxation length and cornering force generation are needed. This means using non-steadystate properties to predict the behaviour of the tires, thereby making the tire model the most complex part of the simulation. Smiley and Horne [34] have compiled static and dynamic properties for modern aircraft tires and curve-fitted empirical formulas to the data. Some other less complete studies on tires have also been carried out by NASA and discussed in References [35] and [36].

Conventional vehicle simulations assume negligible deflections at low speeds and assume no lag in the tire force generation when turning at higher speeds. The effects of relaxation length and cornering force generation are essentially ignored because they occur so quickly 
at high speeds. This was demonstrated by Sleeper and Smith [37] in their airplane landing simulation, which is presented in Section 1.3.3.

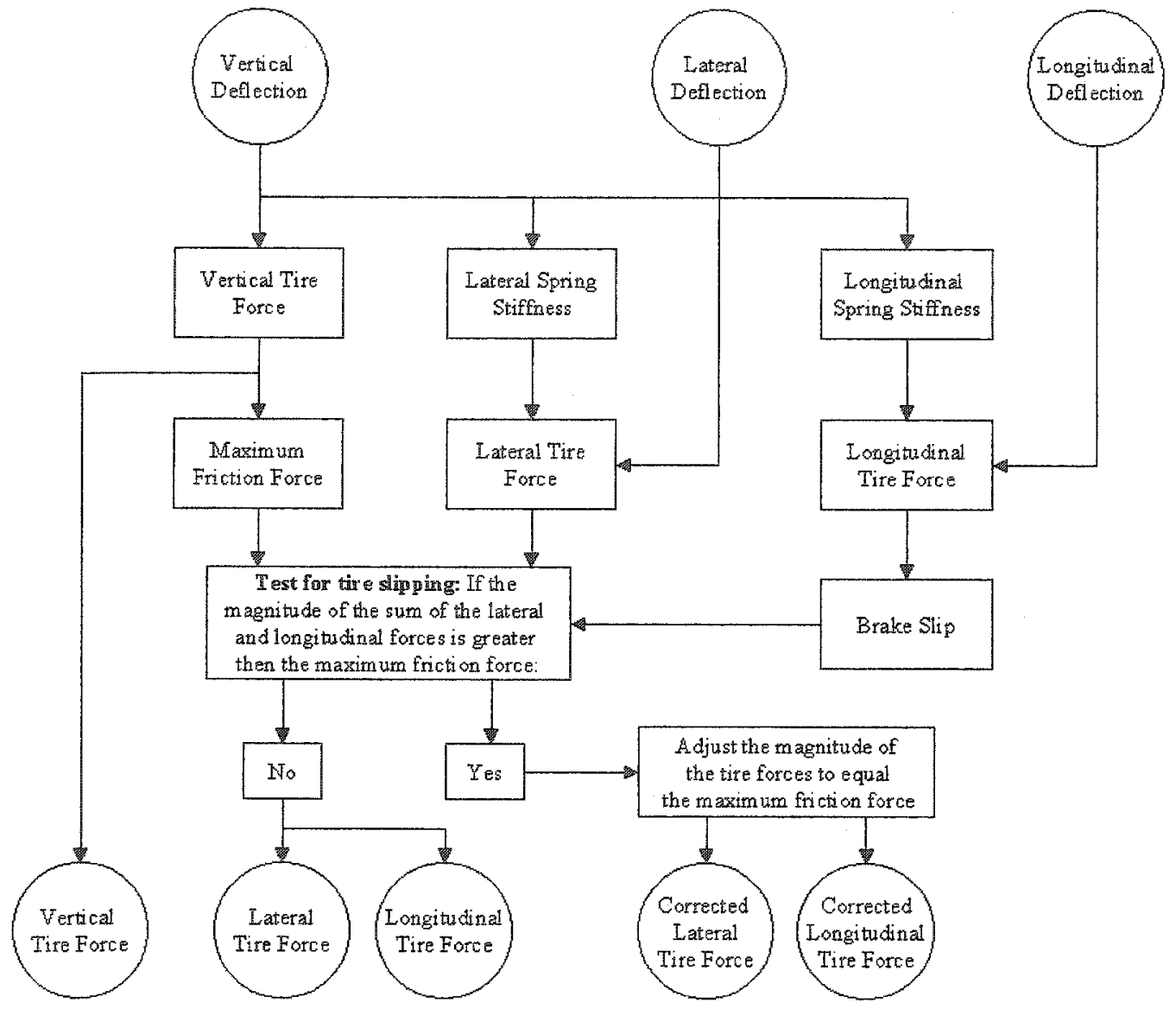

Figure 1.6: Dynaface ${ }^{\circledR}$ tire model

A widely used empirical tire model is the magic formula developed by Pacejka, which is presented in its newest form by Pacejka and Besselink [38]. The magic formula is a method for curve fitting experimental tire data. This can include cornering force versus slip angle, self-aligning torque versus slip angle, as well as braking effort versus skid. The magic formula is a good method for modelling tires, but at this time it has not been applied to aircraft tires. Van Slagmaat [39] outlines what needs to be done to apply the magic formula to airplane landing gear. It would go beyond the scope of this project to carry out the work needed to obtain the coefficients to use the magic formula. For this work the 
NASA model will be used in the helicopter manoeuvring simulation.

A number of nonempirical tire models where also reviewed. Over the past few years, developments in analytical and finite element representations of tires have increased in popularity with greater computing power available. Two examples of analytical tire models can be found in References [40] and [41]. The analytical solutions use springs in spokelike configurations to model the tire. Three finite element models (FEM) are presented as References [42], [43] and [44]. The FEM models calculate the deflections in the entire tire, which would be impractical for a real-time simulation. Another reason why these models have not been considered for the manoeuvring simulation is the lack of the required parameter values required to implement the models.

\subsubsection{Airplane Taxiing}

Some aspects of taxiing airplanes have been found to be very similar to a manoeuvring helicopter. Both move with non-driven wheels, a taxiing airplane is propelled by engines or towed by a small tractor and the helicopter is manoeuvred by forces applied at the wheels or by a probe attached to the fuselage of the helicopter. However, it has been found that these studies emphasize the high speed landing phase of a taxiing airplane. The taxiing of an airplane was the subject of two significant studies: a Ph.D. thesis conducted by Hogg [45]; and two technical reports produced at NASA by Sleeper and Smith [37, 46].

Hogg's thesis was completed to improve the taxiing capabilities of a flight simulator. Hogg used the tire model presented by Smiley and Horne [34]. This is the same model that will be used for the helicopter manoeuvring simulation, but no clear results or method of application are presented by Hogg. A number of other papers have been written by the same group of researchers investigating the taxiing of airplanes in References [47], [48], and [49].

Sleeper and Smith present their experimental work in the first report [46] and a very 
thorough simulation in the second report [37]. The purpose of the NASA study was to test the parametric effects of an airplane landing in a crosswind. Although the application is very similar between this project and the NASA study, there are some pivotal differences: the study was done for a landing airplane experiencing speeds up to twenty times faster than a traversing helicopter; due to the high speeds, a time-lag tire model was applied and found to have an insignificant effect; and the equations of motion where derived using Lagrange's Equation with no reaction forces determined at the castor wheel pivot point that are necessary to drive a joint friction model. The Sleeper and Smith study will be referred to throughout this thesis.

\subsection{Objectives}

It has been shown that an on-deck manoeuvring simulation capability for the helicopter/ship dynamic interface is not currently available. The specific objectives for this project are as follows:

1. To develop an interactive planar on-deck helicopter manoeuvring simulation for the on-deck straightening of helicopters using the ASIST system.

2. To validate and identify any limitations found in the resulting simulation using experimental data.

The primary objective is to develop a simulation called HeliMan, that uses transient mathematical models for: the helicopter equations of motion; the tires; the RSD/probe interface and; the optional castoring auxiliary landing gear. In particular, the castoring wheel model is a unique challenge that must be overcome. All of these models must be interfaced properly to produce an accurate simulation model. This is all done without the presence of ship motion. 
The 'interactive' part of HeliMan will also be the first step in the development of an ASIST training simulator. To accomplish this HeliMan has to have the ability to be controlled by a joystick with simple graphical feedback on the computer screen. It must also be able to be controlled using an input file for testing the capabilities of the system.

The simulation will only model ITI's ASIST handling system. It is important that the simulation can model other on-deck handling systems in the future, such as ITI's RAST system which uses tail cables to align the helicopter and the TC-ASIST system that secures the helicopter at the main wheel axles. Other system options, such as a tail probe and nose wheel tray, which keep the helicopter straight while it is being traversed into the hangar, while important, are beyond the scope of this initial phase.

HeliMan will be validated using experimental data from ITI's Integrated Test Facility (ITF). Output from HeliMan will be compared against the experimental data for accuracy. Any discrepancies between the simulation and the experimental data will be discussed thoroughly. An attempt will be made to fix any problems found in the simulation, but if they can not be fixed within the scope of this project, future courses of action will be suggested.

HeliMan will be developed to handle most helicopter landing gear configuration, but the Dead-Load Test Vehicle (DLTV) used on ITI's ITF is configured as a Sikorsky SH-60B Seahawk. Therefore, although the simulation has been developed for different helicopter configurations, experimental validation will be based on the Seahawk configuration.

\subsection{Thesis Overview}

This chapter presented a brief history of helicopter securing and handling along with an introduction to the problem that is the focus of this project. A number of topics relevant to the project have been reviewed and the motivation and objectives for the project were 
stated. Chapter 2 describes the mathematical models used in HeliMan. These include: the helicopter's equations of motion; the tire model; the RSD/probe model; the optional castor joint model; the simulation model and; the numerical methods. Chapter 3 outlines the validation process used and presents the results from this process. Any changes that needed to be made to the simulation are discussed. Chapter 4 contains concluding remarks with recommendations for future work. 


\section{Chapter 2}

\section{Mathematical Model}

An interactive planar helicopter manoeuvring simulation called HeliMan has been created that models the ASIST system and associated helicopter handling behaviour. The simulation includes a transient three-degree-of-freedom tire model for each tire, and accurate helicopter planar dynamics including an option for a castorable or steerable nose or tail wheel. The mathematical formulation behind the essential elements and numerical methods used in HeliMan are described in this chapter. A detailed description of how to use HeliMan is presented in Appendix A.

The mathematical model has been broken into three parts: helicopter dynamics; simulation control; and simulation structure and solution. The helicopter dynamics model includes all the elements pertaining to the helicopter including: the equations of motion; the tire model; the aerodynamic model and; the RSD/Probe model. The control model describes the different ways the simulation can be controlled, such as with an interactive joystick, input file or algorithm. The simulation model describes how all of the elements are interfaced and how the numerical methods are used to obtain solutions. 


\subsection{Helicopter Dynamics Model}

The generic helicopter on which the model is based is shown in Figure 2.1. It consists of two bodies representing the fuselage and the rotatable wheel assembly interconnected by a revolute joint located at point $B$; and three suspension stations each having either single or dual wheels. The geometry of the system is completely arbitrary with no implied assumptions of symmetry.

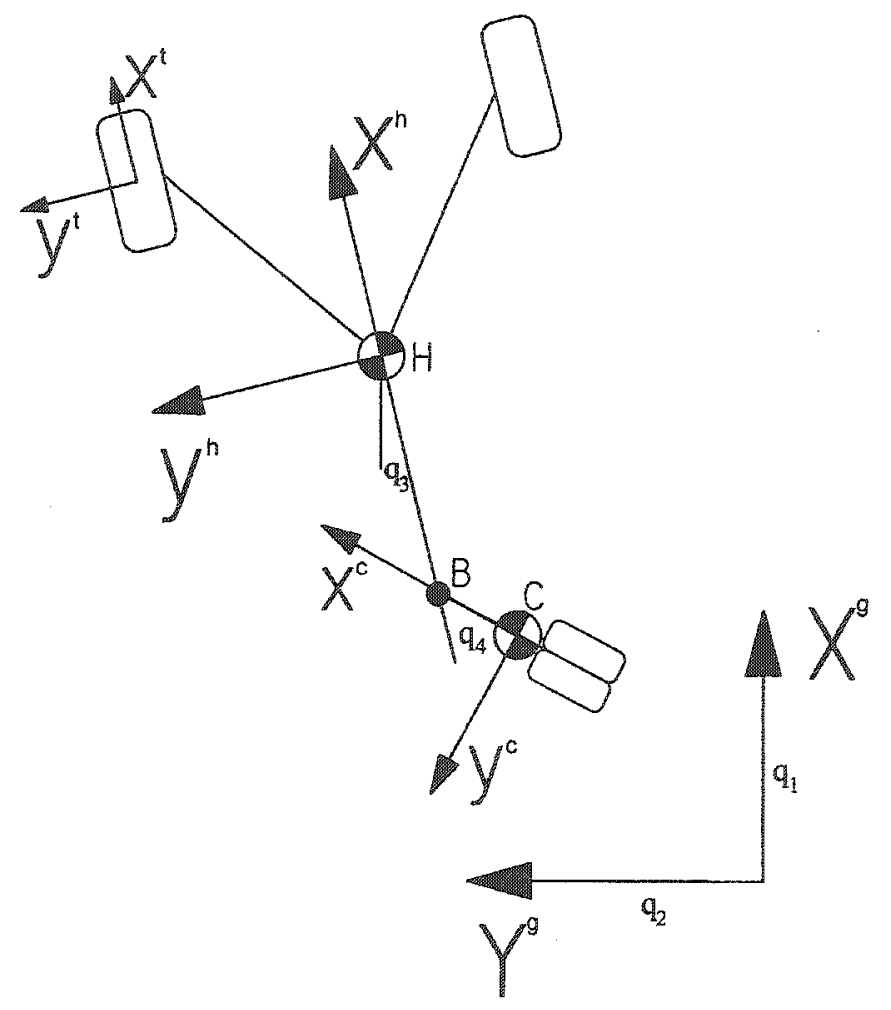

Figure 2.1: Schematic of the dynamic helicopter model where, $\mathrm{H}$ is the main helicopter body's centre of mass and C is the castor assembly's centre of mass

Figure 2.1 illustrates the four degrees of freedom included in the helicopter model, where the generalized coordinates $q_{i}$ are: the linear x position of the helicopter centre of mass $(\mathrm{CM})\left(q_{1}\right)$; the linear y position of the helicopter $\mathrm{CM}\left(q_{2}\right)$; the orientation of the helicopter $\left(q_{3}\right)$; and the orientation of the optionally castorable wheel assembly relative to the helicopter body $\left(q_{4}\right)$. 


\subsubsection{Equations of Motion}

In this section the core governing equations for the dynamic helicopter model are derived. They are structured to allow the extraction of joint reaction forces at the castoring point required by a joint friction model, and such that only ordinary differential equations result, thereby permitting efficient computer implementation without requiring the solution of complex differential-algebraic equations. This was done using the Newton-Euler method for deriving the equations along with a general kinematic analysis of the system.

Sleeper and Smith [37] derived equations of motion for a similar three-wheeled aircraft using Lagrange's method. Lagrange's formulation does not explicitly consider the internal forces of a multi-body system. Newton-Euler equations have been used in order to use the internal joint forces to apply friction at the castor joint. Although Sleeper and Smith derived their equations generally for castoring wheels, they controlled the steering angles for their airplane testing. As a result, for their application joint reaction forces and joint friction could be ignored.

\section{Kinematic Analysis}

First, the kinematic analysis of the two body system was performed. Kinematic analysis of the system allows the inertial frame accelerations of the helicopter body and castor assembly to be related to the generalized coordinates and their first and second time derivatives.

The kinematic representation of the helicopter body, refer to body $\mathrm{H}$ in Figure 2.1, is trivial. The linear position of helicopter body is defined as a vector in Equation 2.1. The orientation of the helicopter body is defined directly by the state variable $q_{3}$.

$$
\vec{R}_{H}^{g}=q_{1} \hat{i}+q_{2} \hat{j}
$$


Equation 2.1 is differentiated once to obtain the velocity vector, $\vec{V}_{H}^{g}=\dot{q}_{1} \hat{i}+\dot{q}_{2} \hat{j}$, and a second time to obtain the acceleration vector, $\vec{A}_{H}^{g}=\ddot{q}_{1} \hat{i}+\ddot{q}_{2} \hat{j}$. The orientation is also differentiated to obtain the angular velocity, $\dot{q}_{3}$, and acceleration, $\ddot{q}_{3}$.

The three global planar accelerations $A_{H x}^{g}, A_{H y}^{g}$ and $\alpha_{H}$, are equal to the second time derivatives of the first three state variables $\ddot{q}_{1}$ to $\ddot{q}_{3}$ respectively.

The most intricate part of the kinematic analysis was describing the castor using the generalized coordinates. With only four degrees of freedom the linear position coordinates locating the castor are redundant. Therefore, the castor assembly was analyzed separately, starting with its global position vector:

$$
\vec{R}_{C}^{g}=\vec{R}_{H}^{g}+\left[T^{h \rightarrow g}\right] \vec{R}_{B / H}^{h}+\left[T^{c \rightarrow g}\right] \vec{R}_{B / C}^{c}
$$

When expanded Equation 2.2 results in:

$$
\begin{aligned}
\vec{R}_{C}^{g} & =\left(q_{1}+R_{B / H_{x}}^{h} \cos q_{3}-R_{B / H_{y}}^{h} \sin q_{3}-R_{B / C_{x}}^{c} \cos \left(q_{3}+q_{4}\right)+R_{B / C_{y}}^{c} \sin \left(q_{3}+q_{4}\right)\right) \hat{i} \\
& +\left(q_{2}+R_{B / H_{x}}^{h} \sin q_{3}+R_{B / H_{y}}^{h} \cos q_{3}-R_{B / C_{x}}^{c} \sin \left(q_{3}+q_{4}\right)-R_{B / C_{y}}^{c} \cos \left(q_{3}+q_{4}\right)\right) \hat{j}
\end{aligned}
$$

Differentiating the position vector, Equation 2.3, results in the velocity vector:

$$
\begin{aligned}
\vec{V}_{C}^{g}= & \left(\dot{q}_{1}-R_{B / H_{x}}^{h} \sin q_{3} \dot{q}_{3}-R_{B / H_{y}}^{h} \cos q_{3} \dot{q}_{3}+R_{B / C_{x}}^{c} \sin \left(q_{3}+q_{4}\right)\left(\dot{q}_{3}+\dot{q}_{4}\right)\right. \\
& \left.+R_{B / C_{3}}^{c} \cos \left(q_{3}+q_{4}\right)\left(\dot{q}_{3}+\dot{q}_{4}\right)\right) \hat{i} \\
+ & \left(\dot{q}_{2}+R_{B / H_{x}}^{h} \cos q_{3} \dot{q}_{3}-R_{B / H_{y}}^{h} \sin q_{3} \dot{q}_{3}-R_{B / C_{x}}^{c} \cos \left(q_{3}+q_{4}\right)\left(\dot{q}_{3}+\dot{q}_{4}\right)\right. \\
& \left.+R_{B / C_{3}}^{c} \sin \left(q_{3}+q_{4}\right)\left(\dot{q}_{3}+\dot{q}_{4}\right)\right) \hat{j}
\end{aligned}
$$


Differentiating the velocity vector, Equation 2.4, results in the acceleration vector:

$$
\begin{aligned}
\vec{A}_{C}^{g}= & \left(\ddot{q}_{1}-R_{B / H_{x}}^{h} \sin q_{3} \ddot{q}_{3}-R_{B / H_{x}}^{h} \cos q_{3} \dot{q}_{3}^{2}-R_{B / H_{y}}^{h} \cos q_{3} \ddot{q}_{3}+R_{B / H_{y}}^{h} \sin q_{3} \dot{q}_{3}^{2}\right. \\
& +R_{B / C_{x}}^{c} \sin \left(q_{3}+q_{4}\right)\left(\ddot{q}_{3}+\ddot{q}_{4}\right)+R_{B / C_{x}}^{c} \cos \left(q_{3}+q_{4}\right)\left(\dot{q}_{3}+\dot{q}_{4}\right)^{2} \\
& \left.+R_{B / C_{y}}^{c} \cos \left(q_{3}+q_{4}\right)\left(\ddot{q}_{3}+\ddot{q}_{4}\right)-R_{B / C_{y}}^{c} \sin \left(q_{3}+q_{4}\right)\left(\dot{q}_{3}+\dot{q}_{4}\right)^{2}\right) \hat{i} \\
+ & \left(\ddot{q}_{2}+R_{B / H_{x}}^{h} \cos q_{3} \ddot{q}_{3}-R_{B / H_{x}}^{h} \sin q_{3} \dot{q}_{3}^{2}-R_{B / H_{y}}^{h} \sin q_{3} \ddot{q}_{3}-R_{B / H_{y}}^{h} \cos q_{3} \dot{q}_{3}^{2}\right. \\
& -R_{B / C_{x}}^{c} \cos \left(q_{3}+q_{4}\right)\left(\ddot{q}_{3}+\ddot{q}_{4}\right)+R_{B / C_{x}}^{c} \sin \left(q_{3}+q_{4}\right)\left(\dot{q}_{3}+\dot{q}_{4}\right)^{2} \\
& \left.+R_{B / C_{y}}^{c} \sin \left(q_{3}+q_{4}\right)\left(\ddot{q}_{3}+\ddot{q}_{4}\right)+R_{B / C_{y}}^{c} \cos \left(q_{3}+q_{4}\right)\left(\dot{q}_{3}+\dot{q}_{4}\right)^{2}\right) \hat{j}
\end{aligned}
$$

Equation 2.5 represents the global linear accelerations of the castor assembly centre of mass, $A_{C x}^{g}$ and $A_{C y}^{g}$ in terms of the four state variables, $q_{1}$ to $q_{4}$ and their time derivatives. The global angular acceleration of the castor body, $\alpha_{C}$, is equal to the helicopter body's angular acceleration plus the relative acceleration of the castor assembly:

$$
\alpha_{C}=\ddot{q}_{3}+\ddot{q}_{4}
$$

The final kinematic equations, combining the helicopter and castor kinematics, are presented in matrix form as:

$$
\left\{\begin{array}{c}
A_{H x}^{g} \\
A_{H y}^{g} \\
\alpha_{H} \\
A_{C x}^{g} \\
A_{C y}^{g} \\
\alpha_{C}
\end{array}\right\}=\left[\begin{array}{cccc}
1 & 0 & 0 & 0 \\
0 & 1 & 0 & 0 \\
0 & 0 & 1 & 0 \\
1 & 0 & B_{43} & B_{44} \\
0 & 1 & B_{53} & B_{54} \\
0 & 0 & 1 & 1
\end{array}\right]\left\{\begin{array}{c}
\ddot{q}_{1} \\
\ddot{q}_{2} \\
\ddot{q}_{3} \\
\ddot{q}_{4}
\end{array}\right\}+\left\{\begin{array}{c}
0 \\
0 \\
0 \\
C_{4} \\
C_{5} \\
0
\end{array}\right\}
$$


where,

$$
\begin{aligned}
& B_{43}=-R_{B / H_{x}}^{h} \mathrm{~s} q_{3}-R_{B / H_{y}}^{h} \mathrm{c} q_{3}+R_{B / C_{x}}^{c} \mathrm{~s}\left(q_{3}+q_{4}\right)+R_{B / C_{y}}^{c} \mathrm{c}\left(q_{3}+q_{4}\right) \\
& B_{44}=R_{B / C_{x}}^{c} \mathrm{~s}\left(q_{3}+q_{4}\right)+R_{B / C_{3}}^{c} \mathrm{c}\left(q_{3}+q_{4}\right) \\
& B_{53}=R_{B / H_{x}}^{h} \mathrm{c} q_{3}-R_{B / H_{y}}^{h} \mathrm{~s} q_{3}-R_{B / C_{x}}^{c} \mathrm{c}\left(q_{3}+q_{4}\right)+R_{B / C_{y}}^{c} \mathrm{~s}\left(q_{3}+q_{4}\right) \\
& B_{54}=-R_{B / C_{x}}^{c} \mathrm{c}\left(q_{3}+q_{4}\right)+R_{B / C_{y}}^{c} \mathrm{~s}\left(q_{3}+q_{4}\right) \\
& C_{4}=-R_{B / H_{x}}^{h} \mathrm{c} q_{3} \dot{q}_{3}^{2}+R_{B / H_{y}}^{h} \mathrm{~s} q_{3} \dot{q}_{3}^{2}+R_{B / C_{x}}^{c} \mathrm{c}\left(q_{3}+q_{4}\right)\left(\dot{q}_{3}+\dot{q}_{4}\right)^{2}-R_{B / C_{y}}^{c} \mathrm{~s}\left(q_{3}+q_{4}\right)\left(\dot{q}_{3}+\dot{q}_{4}\right)^{2} \\
& C_{5}=-R_{B / H_{x}}^{h} \mathrm{~s} q_{3} \dot{q}_{3}^{2}-R_{B / H_{y}}^{h} \mathrm{c} q_{3} \dot{q}_{3}^{2}+R_{B / C_{x}}^{c} \mathrm{~s}\left(q_{3}+q_{4}\right)\left(\dot{q}_{3}+\dot{q}_{4}\right)^{2}+R_{B / C_{y}}^{c} \mathrm{c}\left(q_{3}+q_{4}\right)\left(\dot{q}_{3}+\dot{q}_{4}\right)^{2}
\end{aligned}
$$

and where $\mathrm{c}$ and $\mathrm{s}$ represent cosine and sine functions respectively. For subsequent analysis, Equation 2.7 can be written more compactly as:

$$
\{A\}=[B]\{\ddot{q}\}+\{C\}
$$

where individual terms are identified by comparison with Equation 2.7.

\section{Newton-Euler Analysis}

Next, the Newton-Euler method is used to take into account the applicable forces and moments and derive the helicopter dynamics. Free body diagrams for each body are shown in Figure 2.2. All of the applicable forces and moments acting on each body are shown. The forces and moments acting on the main helicopter body are: the tire forces $\left(\vec{F}_{m l t_{1}}, \vec{F}_{m l t_{2}}, \vec{F}_{m r t_{1}}\right.$ and $\left.\vec{F}_{m r t_{2}}\right) ;$ the forces acting on the probe $\left(\vec{F}_{P}\right) ;$ the aerodynamic forces $\left(\vec{F}_{d x}\right.$ and $\left.\vec{F}_{d y}\right)$; and the reaction force $\left(\vec{F}_{B}\right)$ and moment $\left(M_{B}\right)$ at the pivot joint. The forces and moments acting on the castor assembly are: the tire forces $\left(\vec{F}_{c t_{1}}\right.$ and $\left.\vec{F}_{c t_{2}}\right)$; and the reaction force $\left(\vec{F}_{B}\right)$ and moment $\left(M_{B}\right)$ at the pivot joint. These forces and corresponding moments about the centres of mass have been dynamically balanced in Equations 2.9 to 2.14 with the application of Newton-Euler equations in the global frame of reference. 

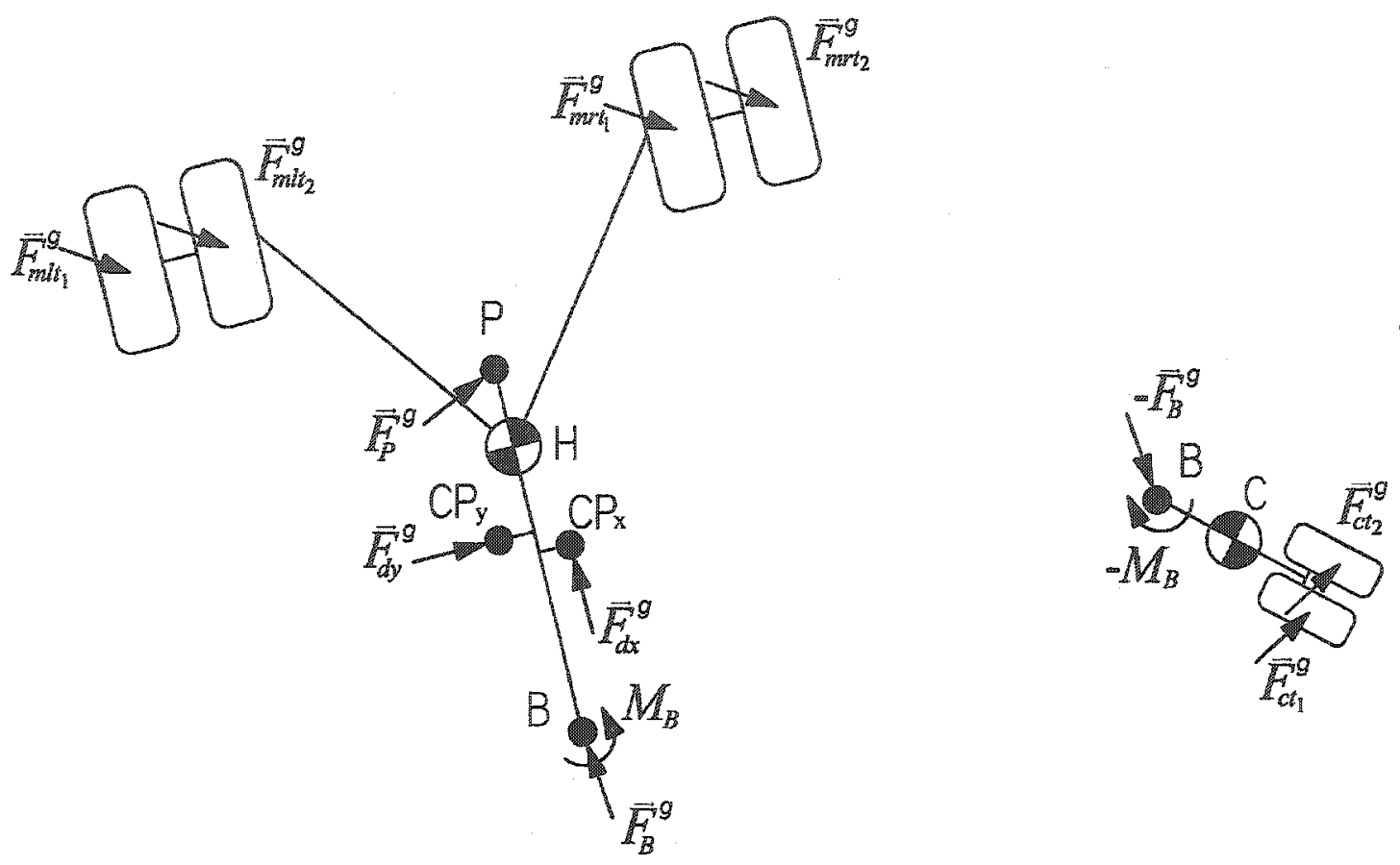

Figure 2.2: Free-body diagram of the main helicopter body and castorable wheel assembly.

$$
\begin{gathered}
m_{H} A_{H_{x}}^{g}=F_{P_{x}}^{g}+F_{d x_{x}}^{g}+F_{d y_{x}}^{g}+F_{B_{x}}^{g}+F_{m l t_{x 1}}^{g}+F_{m l t_{x 2}}^{g}+F_{m r t_{x 1}}^{g}+F_{m r t_{x 2}}^{g} \\
m_{H} A_{H_{y}}^{g}=F_{P_{y}}^{g}+F_{d x_{y}}^{g}+F_{d y_{y}}^{g}+F_{B_{y}}^{g}+F_{m l t_{y 1}}^{g}+F_{m l t_{y 2}}^{g}+F_{m r t_{y 1}}^{g}+F_{m r t_{y 2}}^{g} \\
I_{H} \alpha_{H}=\vec{R}_{P / H}^{g} \times \vec{F}_{P}^{g}+\vec{R}_{B / H}^{g} \times \vec{F}_{B}^{g}+\vec{R}_{m l t / H_{1}}^{g} \times \vec{F}_{m l t_{1}}^{g}+\vec{R}_{m l t / H_{2}}^{g} \times \vec{F}_{m l t_{2}}^{g} \\
+\vec{R}_{m r t / H_{1}}^{g} \times \vec{F}_{m r t_{1}}^{g}+\vec{R}_{m r t / H_{2}}^{g} \times \vec{F}_{m r t_{2}}^{g}+\vec{R}_{C P x / H}^{g} \times \vec{F}_{d x}^{g}+\vec{R}_{C P y / H}^{g} \times \vec{F}_{d y}^{g}+M_{B} \\
m_{C} A_{C_{x}}^{g}=-\vec{F}_{B_{x}}^{g}+\vec{F}_{c t_{x 1}}^{g}+F_{c t_{x 2}}^{g} \\
m_{C} A_{C_{y}}^{g}=-F_{B_{y}}^{g}+\vec{F}_{c t_{y 1}}^{g}+F_{c t_{y 2}}^{g} \\
I_{C} \alpha_{C}=\vec{R}_{B / C}^{g} \times-\vec{F}_{B}^{g}+\vec{R}_{c t / C_{1}}^{g} \times \vec{F}_{c t_{1}}^{g}+\vec{R}_{c t / C_{2}}^{g} \times \vec{F}_{c t_{2}}^{g}-M_{B}
\end{gathered}
$$


In matrix form, these equations can be expressed as:

$$
\begin{gathered}
{\left[\begin{array}{cccccc}
m_{H} & 0 & 0 & 0 & 0 & 0 \\
0 & m_{H} & 0 & 0 & 0 & 0 \\
0 & 0 & I_{H} & 0 & 0 & 0 \\
0 & 0 & 0 & m_{C} & 0 & 0 \\
0 & 0 & 0 & 0 & m_{C} & 0 \\
0 & 0 & 0 & 0 & 0 & I_{C}
\end{array}\right]\left\{\begin{array}{c}
A_{H x}^{g} \\
A_{H y}^{g} \\
\alpha_{H} \\
A_{C x}^{g} \\
A_{C y}^{g} \\
\alpha_{C}
\end{array}\right\}=} \\
F_{P_{x}}^{g}+F_{d x_{x}}^{g}+F_{d y_{x}}^{g}+F_{B_{x}}^{g}+F_{m l t_{x 1}}^{g}+F_{m l t_{x 2}}^{g}+F_{m r t_{x 1}}^{g}+F_{m r t_{x 2}}^{g}+F_{B_{y}}^{g}+F_{m l t_{y 1}}^{g}+F_{m l t_{y 2}}^{g}+F_{m r t_{y 1}}^{g}+F_{m r t_{y 2}}^{g} \\
\left\{\begin{array}{c}
F_{3} \\
-F_{B_{x}}^{g}+F_{c t_{x 1}}^{g}+F_{c t_{x 2}}^{g}+F_{c t_{y 1}}^{g}+F_{c t_{y 2}}^{g} \\
\vec{R}_{B / C}^{g} \times-\vec{F}_{B}^{g}+\vec{R}_{c t / C_{1}}^{g} \times \vec{F}_{c t_{1}}^{g}+\vec{R}_{c t / C_{2}}^{g} \times \vec{F}_{c t_{2}}^{g}-M_{B}
\end{array}\right\}
\end{gathered}
$$

where,

$$
\begin{aligned}
F_{3} & =\vec{R}_{P / H}^{g} \times \vec{F}_{P}^{g}+\vec{R}_{B / H}^{g} \times \vec{F}_{B}^{g}+\vec{R}_{m l t / H_{1}}^{g} \times \vec{F}_{m l t_{1}}^{g}+\vec{R}_{m l t / H_{2}}^{g} \times \vec{F}_{m l t_{2}}^{g} \\
& +\vec{R}_{m r t / H_{1}}^{g} \times \vec{F}_{m r t_{1}}^{g}+\vec{R}_{m r t / H_{2}}^{g} \times \vec{F}_{m r t_{2}}^{g}+\vec{R}_{C P x / H}^{g} \times \vec{F}_{d x}^{g}+\vec{R}_{C P y / H}^{g} \times \vec{F}_{d y}^{g}+M_{B}
\end{aligned}
$$

Again for subsequent analysis, Equation 2.15 can be written more compactly as:

$$
[M]\{A\}=\{F\}
$$

where individual terms are identified by comparison with Equation 2.15. 


\section{Combined Analysis}

Next, the kinematic analysis is combined with the Newton-Euler analysis to obtain the general equations of motion for the helicopter. Substituting Equation 2.8 into 2.16 we get:

$$
[M]([B]\{\ddot{q}\}+\{C\})=\{F\}
$$

Then the state variables are kept on the left, while everything else is moved to the right:

$$
[M][B]\{\ddot{q}\}=\{F\}-[M]\{C\}
$$

At this point there are six equations with six unknowns. The second time derivatives of the four state variables are unknown, these correspond to the four degrees of freedom of the system, along with the $\mathrm{x}$ and $\mathrm{y}$ components of the reaction force on the castor joint $\left(\vec{F}_{B}^{g}\right)$. The unknown reaction force components must be separated from the known forces and moved to the left-hand side of Equation 2.18. To do this the $\{F\}$ vector is first split into two vectors, $\left\{F_{k}\right\}$ and $\left\{F_{u}\right\}$ :

$$
\{F\}=\left\{F_{k}\right\}+\left\{F_{u}\right\}
$$

Equation 2.19 is substituted back into Equation 2.18 and $\left\{F_{u}\right\}$ is placed on the left hand side as follows:

$$
[M][B]\{\ddot{q}\}-\left\{F_{u}\right\}=\left\{F_{k}\right\}-[M]\{C\}
$$


The unknown forces must next be extracted from the moment expressions:

$$
\left\{F_{u}\right\}=[D]\left\{\begin{array}{c}
F_{B_{x}}^{g} \\
F_{B_{y}}^{g}
\end{array}\right\}
$$

where,

$$
[D]=\left[\begin{array}{cc}
1 & 0 \\
0 & 1 \\
-R_{B / H_{y}}^{g} & R_{B / H_{x}}^{g} \\
-1 & 0 \\
0 & -1 \\
R_{B / C_{y}}^{g} & -R_{B / C_{x}}^{g}
\end{array}\right]
$$

The matrices are merged to isolate all six unknowns on the left side, which results in a set of simultaneous linear equations that can be solved for the unknown accelerations and forces as follows:

$$
[[M][B]-[D]]\left\{\begin{array}{c}
\ddot{q}_{1} \\
\ddot{q}_{2} \\
\ddot{q}_{3} \\
\ddot{q}_{4} \\
F_{B_{x}}^{g} \\
F_{B_{y}}^{g}
\end{array}\right\}=\left\{F_{k}\right\}-[M]\{C\}
$$


The expanded form of Equation 2.23 is:

$$
\begin{aligned}
& {\left[\begin{array}{cccccc}
m_{h} & 0 & 0 & 0 & -1 & 0 \\
0 & m_{h} & 0 & 0 & 0 & -1 \\
0 & 0 & I_{h} & 0 & R_{B / H_{y}}^{g} & -R_{B / H_{x}}^{g} \\
m_{c} & 0 & m_{c} B_{43} & m_{c} B_{44} & 1 & 0 \\
0 & m_{c} & m_{c} B_{53} & m_{c} B_{54} & 0 & 1 \\
0 & 0 & I_{c} & I_{c} & -R_{B / C_{y}}^{g} & R_{B / C_{x}}^{g}
\end{array}\right]\left\{\begin{array}{c}
\ddot{q}_{1} \\
\ddot{q}_{2} \\
\ddot{q}_{3} \\
\ddot{q}_{4} \\
F_{B_{x}}^{g} \\
F_{B_{3}}^{g}
\end{array}\right\}=} \\
& \left\{\begin{array}{c}
F_{P_{x}}^{g}+F_{d x_{x}}^{g}+F_{d y_{x}}^{g}+F_{m l t_{x 1}}^{g}+F_{m l t_{x 2}}^{g}+F_{m r t_{x 1}}^{g}+F_{m r t_{x 2}}^{g} \\
F_{P_{y}}^{g}+F_{d x_{y}}^{g}+F_{d y_{y}}^{g}+F_{m l t_{y 1}}^{g}+F_{m i t_{y 2}}^{g}+F_{m r t_{y 1}}^{g}+F_{m r t_{y 2}}^{g} \\
E_{3} \\
F_{c t_{x 1}}^{g}+F_{c t_{x 2}}^{g}-m_{c} C_{4} \\
F_{c t_{y 1}}^{g}+F_{c t_{y 2}}^{g}-m_{c} C_{5} \\
\vec{R}_{c t / C_{1}}^{g} \times \vec{F}_{c t_{1}}^{g}+\vec{R}_{c t / C_{2}}^{g} \times \vec{F}_{c t_{2}}^{g}-M_{B}
\end{array}\right\}
\end{aligned}
$$

where,

$$
\begin{aligned}
E_{3} & =\vec{R}_{P / H}^{g} \times \vec{F}_{P}^{g}+\vec{R}_{m l t / H_{1}}^{g} \times \vec{F}_{m l t_{1}}^{g}+\vec{R}_{m l t / H_{2}}^{g} \times \vec{F}_{m l t_{2}}^{g}+\vec{R}_{m r t / H_{1}}^{g} \times \vec{F}_{m r t_{1}}^{g} \\
& +\vec{R}_{m r t / H_{2}}^{g} \times \vec{F}_{m r t_{2}}^{g}+\vec{R}_{C P x / H}^{g} \times \vec{F}_{d x}^{g}+\vec{R}_{C P y / H}^{g} \times \vec{F}_{d y}^{g}+M_{B}
\end{aligned}
$$

An analytical solution for Equation 2.24 has been found and presented in Appendix B. While the analytical solution is valid, its complexity, judged by the length of the resulting expressions for the unknown quantities in Equation 2.24, suggest that a numerical solution of Equation 2.24 would be more efficient for realtime simulation than evaluating the analytical expressions. For this reason, Gaussian elimination with partial pivoting has been implemented to solve Equation 2.24. It was found to be fast enough for the simulation to 
run in realtime. Section 2.3.1 describes this numerical process in greater detail.

All of the forces, except for the linear reactions at the joint $\vec{F}_{B}$, acting on the two-body system are determined by the various models outlined in the next sections. These models use many different constants, state variables, and coordinate systems to determine the forces acting on the helicopter.

\subsubsection{Castor Joint Model}

In the free-body diagram shown in Figure 2.2 a reaction moment $M_{B}$ is shown at the pivot joint $\mathrm{B}$. This moment is modelled using two torque contributions that include Coulomb friction $\left(T_{\mu}\right)$ and viscous damping $\left(T_{v}\right)$ shown in Figure 2.3. Equation 2.25 shows the breakdown symbolically as:

$$
M_{B}=T_{\mu}+T_{v}
$$

A third component models the kingpin inclination angle by adding a lateral correcting force, $F_{\kappa}$ to the castor tires. This force is applied to the dynamic equations as part of the force determined by the tire model, $\vec{F}_{c t_{1}}^{g}$ and $\vec{F}_{c t_{1}}^{g}$.

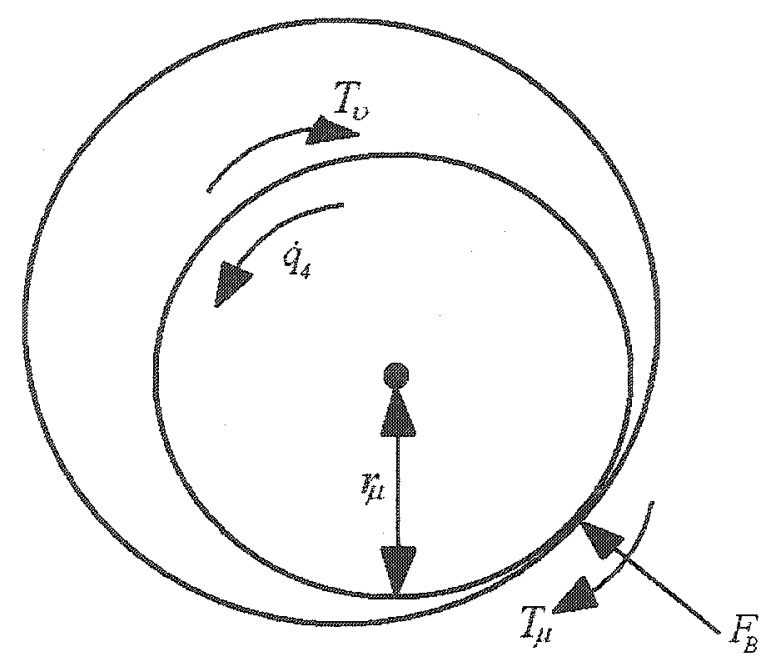

Figure 2.3: Illustration of the Coulomb friction and viscous damping torques acting on the castor joint 
Some helicopter configurations have a tail or nose wheel that can be fixed at a specified angle. The method for fixing the castor assembly in the castor joint model will also be discussed.

\section{Coulomb Friction}

Reaction force $F_{B}$ at the castor pivot point is used to calculate the maximum value of the Coulomb friction contribution acting at the castor joint. This is calculated using Equation 2.26:

$$
T_{\mu}=-\operatorname{sign}\left(\dot{q}_{4}\right)\left(\mu_{B} F_{B} r_{\mu}\right)\left(1-e^{-\beta\left|\dot{q}_{1}\right|}\right)
$$
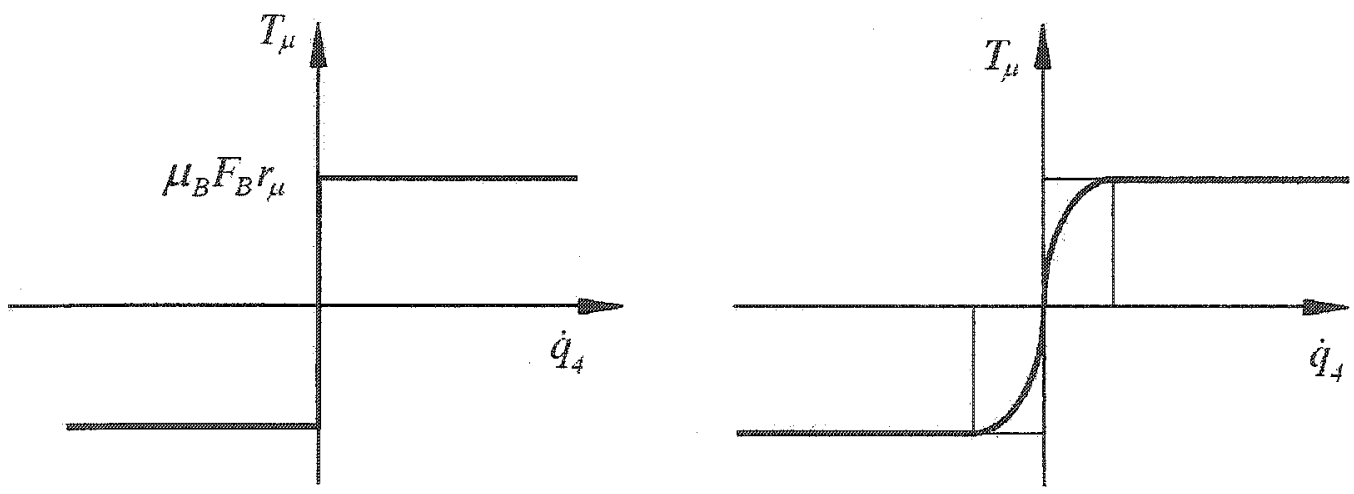

Figure 2.4: Standard (left) and modified (right) Coulomb friction models

The standard Coulomb friction model has been modified using a smoothing function to create a smooth transition when the velocity passes through zero as compared with the conventional bang-bang friction model. Figure 2.4 illustrates this modified Coulomb friction model. This smoothing function is also applied later in Section 2.1.3 to the rolling resistance model.

The determined joint torque acts in the opposite direction of the castor assembly's angular velocity. This is illustrated in Figure 2.3. 


\section{Viscous Damping}

The maximum viscous damping component is proportional to the relative angular velocity of the castor assembly $\left(\dot{q}_{4}\right)$. This is calculated using Equation 2.27:

$$
T_{v}=C_{B} \dot{q}_{4} r_{\mu}^{2}
$$

This torque also acts in the opposite direction of the castor assembly's angular velocity. This is also illustrated in Figure 2.3.

\section{Kingpin Inclination}

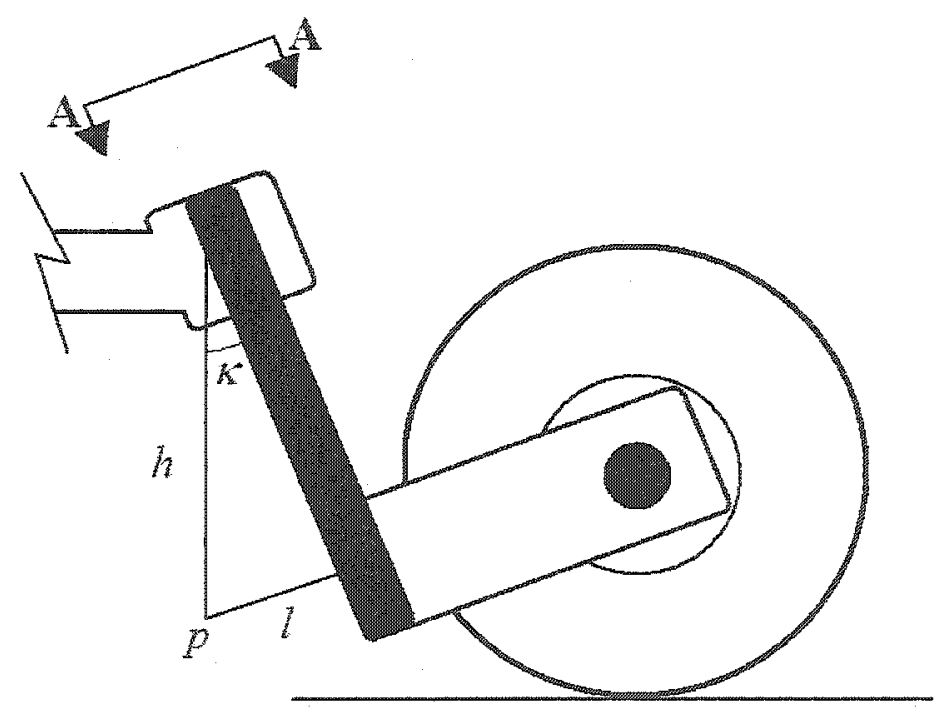

Figure 2.5: Kingpin inclination angle $(\kappa)$

Some helicopter designs include a small inclination in the rotational axis of the castor joint. This is known as the kingpin inclination angle and it creates an aligning torque to help align the castor assembly. Figure 2.5 illustrates an exaggerated kingpin inclination angle. A lateral force on the tires is created based on the rotation angle of the castor and the associated tendency to raise the associated helicopter. The straightening force is 
calculated in Equation 2.28, using the normal force acting on the wheel and the geometry of the castor assembly.

$$
F_{\kappa}=-F_{t_{z}} \sin \alpha
$$

where,

$$
\sin \alpha=\sin \kappa \sin q_{4}
$$

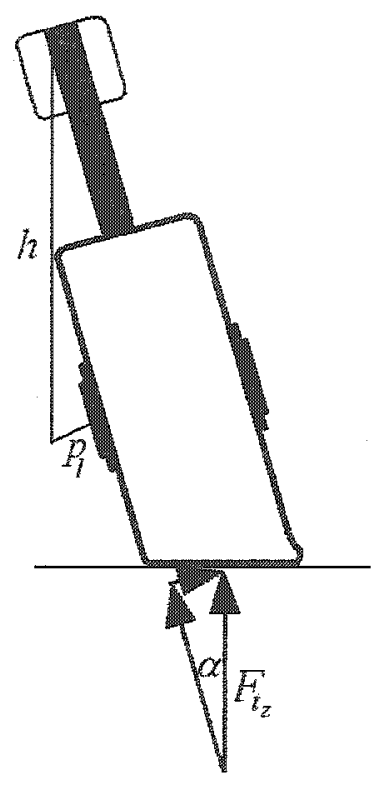

Figure 2.6: Rotated castor wheel demonstrating the effect of the kingpin inclination angle

Equation 2.29 is determined by finding a relationship between the wheel angle $\alpha$, the kingpin inclination angle $\kappa$ and the castor position $q_{4}$. Figure 2.6 illustrates the angle the wheel experiences based on its position.

The first relationship needed is Equation 2.30 and is found from Figure 2.5:

$$
l=h \sin \kappa
$$

The next relationship is found by viewing the castor assembly perpendicular to the 
AA plane defined in Figure 2.5. This view is illustrated in Figure 2.7. The length $l$ is projected onto the other plane perpendicular to the AA plane as $p_{l}$. The projected length is a function of $q_{4}$, as shown in Equation 2.31:

$$
p_{l}=l \sin q_{4}
$$

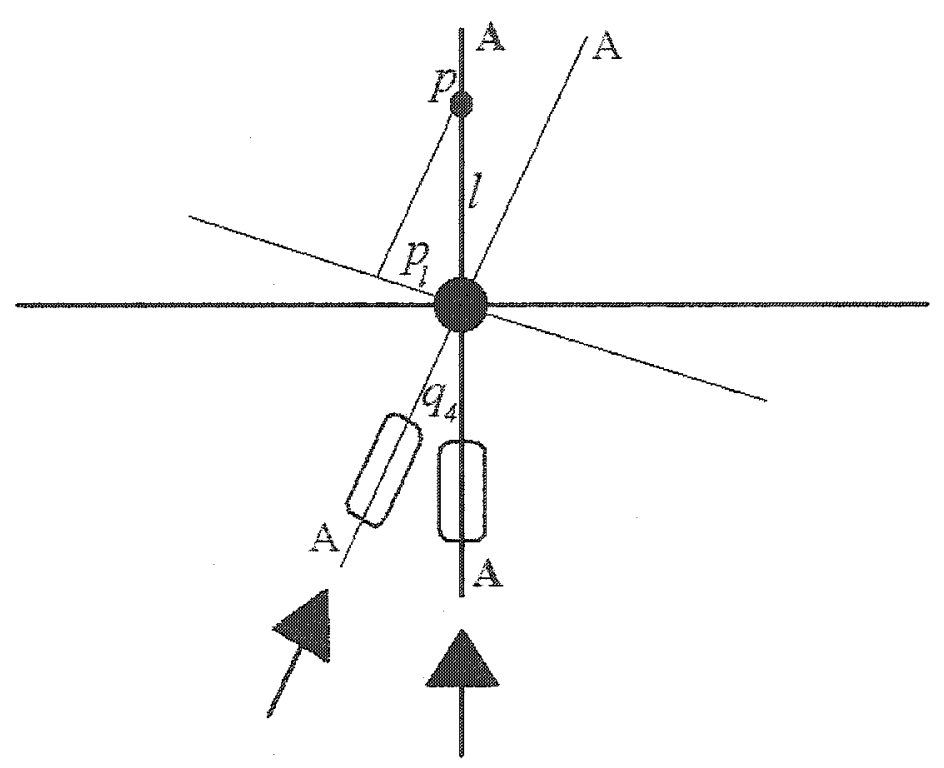

Figure 2.7: AA view of the castor assembly

This projected length can also be viewed perpendicular to the plane it is projected on and is shown in Figure 2.6. Length $p_{l}$ is also found to be proportional to the wheel angle, $\alpha$, with Equation 2.32:

$$
p_{l}=h \sin \alpha
$$

Equations 2.30 to 2.32 are algebraically manipulated to obtain Equation 2.29. It is interesting to note how the expression is independent of the lengths defined to derive it. Although the kingpin force has been derived for only one tire, due to symmetry, it is applicable for the dual-tire case. 


\section{Fixing the Castor Assembly}

The option of fixing the castor assembly at a particular angle was needed to make the simulation flexible enough to model different helicopter landing gear configurations. Effectively locking the castor was accomplished by applying a torsional resistance with a very large stiffness and damping coefficient at the castor joint to counteract any motion. The castor joint moment, $M_{B}$, therefore is calculated differently when the castor assembly is fixed.

$$
M_{B}=K_{f i x} \delta q_{4}+C_{f i x} \dot{q}_{4}
$$

where $\delta q_{4}$ is the change in the rotational position of the castor assembly relative to an arbitrarily specified angle. $M_{B}$ is positive because it was defined to act in the negative direction on the castor assembly.

\subsubsection{Tire Model}

During manoeuvring, the helicopter tires roll at relatively low speed compared with conventional vehicle applications. The low speed complicates the tire model considerably. When modelling a slow-moving tire, as in this application, accurate methods of modelling relaxation length and cornering force generation are needed. This means using non-steadystate properties to predict the behaviour of the tires, thereby making the tire model the most complex part of the simulation. Smiley and Horne [34] present methods for modelling the non-steady-state properties of a rolling aircraft tire.

A three-degree-of-freedom tire model has been constructed using the properties outlined by Smiley and Horne [34]. Figure 2.8 is a flowchart representation of the structure of the tire model. No motion in the vertical direction is considered in the planar simulation, though the vertical tire deflections are automatically calculated such that they are consistent with the helicopter weight distribution. In the longitudinal direction, a rolling 
resistance model is applied.

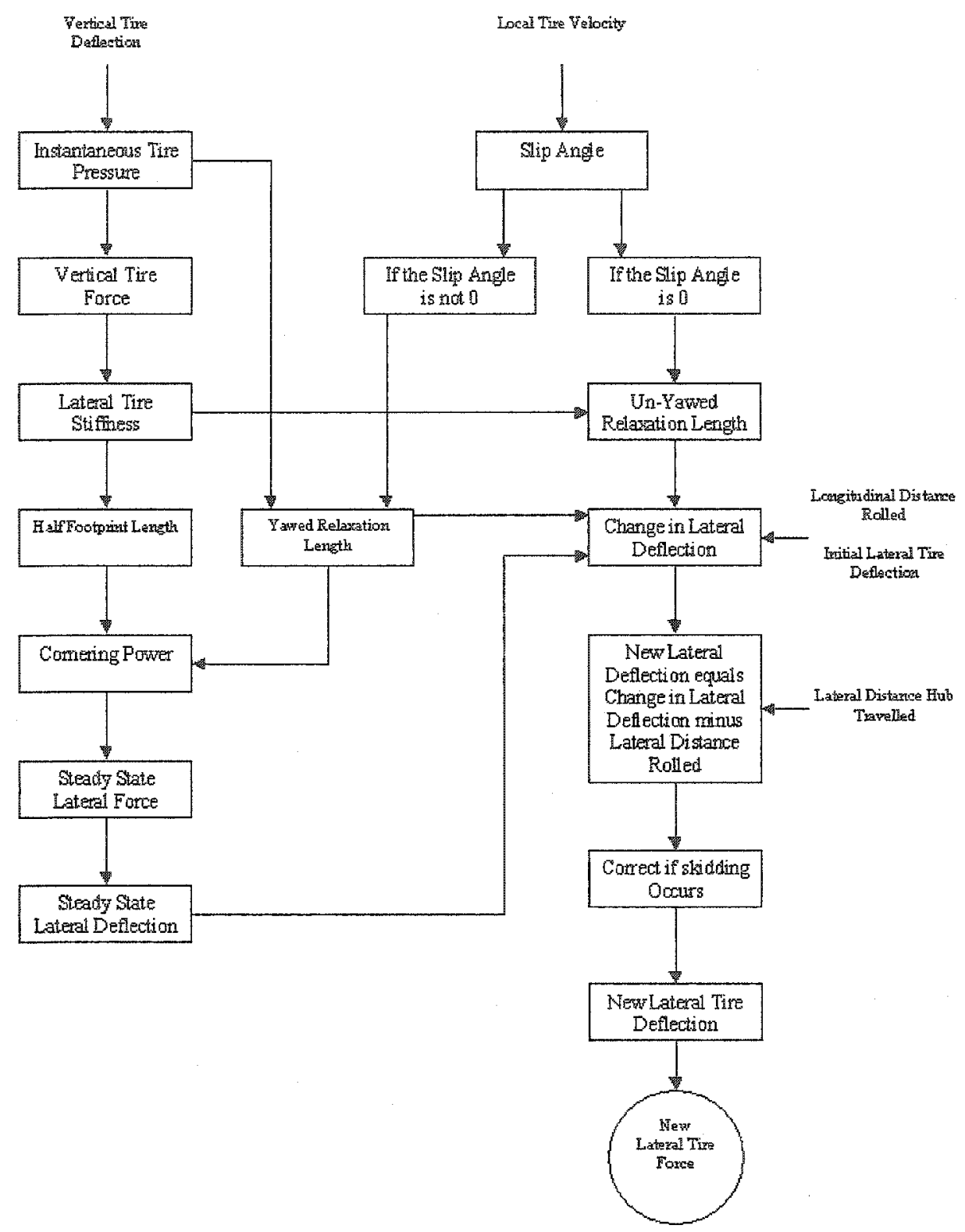

Figure 2.8: Flowchart of the implemented tire model

The lateral direction is much more complex and involves calculating the generation and relaxation of the lateral deflection. The lateral deflection is what creates the cornering force in the tire and can be generated (comering power generation) by giving the tire a velocity component in the lateral direction as it is rolling. This lateral deflection will relax when the 
tire is rolling with no lateral velocity component. The rate at which the lateral deffection is generated or relaxed is based on the distance the tire rolls to reach a specific lateral deflection.

These transient periods of relaxation and generation are so short in high speed applications they are often ignored and a time lag is implemented in the tire model to capture their ultimate effect. This was found to be true by Sleeper and Smith in their testing of a time lag model in Reference [37]. Next the elements of the tire model presented in Figure 2.8 are considered.

\section{Initial Conditions}

The tire model relies on an iterative process to accurately represent the tire behaviour. This can be applied very easily in the numerical solution type used to run the simulation that will be described in Section 2.3. At each time the tire model is called, new input conditions exist. These include: the vertical tire deflection; the local tire velocity; the lateral and longitudinal distances the wheel hub has travelled; and the previous lateral tire deflection.

In the planar simulation, all of these parameters change with the motion of the helicopter, except for the vertical tire deflection. Therefore, the vertical tire deflection is calculated only once when the tire loads are first determined. In the presence of ship motion this deflection would have to be recalculated with each call to the tire model.

\section{Instantaneous Tire Pressure}

First, the instantaneous tire pressure must be calculated so that it is available to determine other parameters later in the model. Smiley and Horne [34] present the following empirical relationship between the vertical deflection and the instantaneous tire pressure shown as 
Equation 2.34.

$$
P=P_{o}+\Delta P
$$

where the change in tire pressure, $\Delta P$ is a function of the tire's vertical deffection, $d_{z}$ and width, $w_{t}$ :

$$
\Delta P=n S_{t} P_{o a}\left(\frac{d_{z}}{w_{t}}\right)^{2}
$$

and the tire shape constant, $S_{t}$ is a function of the tire width, $w_{t}$ and radius $r_{t}$ :

$$
S_{t}=\frac{1.5 w_{t}}{2 r_{t}}
$$

It is assumed that the air-compression process is isothermal with $n$ being equal to 1 . The instantaneous tire pressure is constant for the planar simulation.

Smiley and Horne [34] also consider the change in tire pressure caused by the lateral deflection. The change in pressure due to the lateral deflection has been found to be insignificant when compared to the pressure change caused by the vertical deflection.

\section{Vertical Tire Force}

Since for the planar simulation the vertical tire deflection is constant, the corresponding vertical tire force will also be constant. Though constant in the current application, the tire model has been implemented for the more general case of nonconstant vertical load such that it can be implemented directly in a subsequent three-dimensional simulation model. Figure 2.9 illustrates a tire under an exaggerated vertical load.

Smiley and Horne [34] have derived an empirical relationship between the vertical force and the vertical deflection stating that:

$$
F_{t_{z}}=\left[P+0.08 P_{r}\right] w_{t} \sqrt{w_{t} 2 r_{t}} f_{1}\left(\frac{d_{z}}{w_{t}}\right)
$$




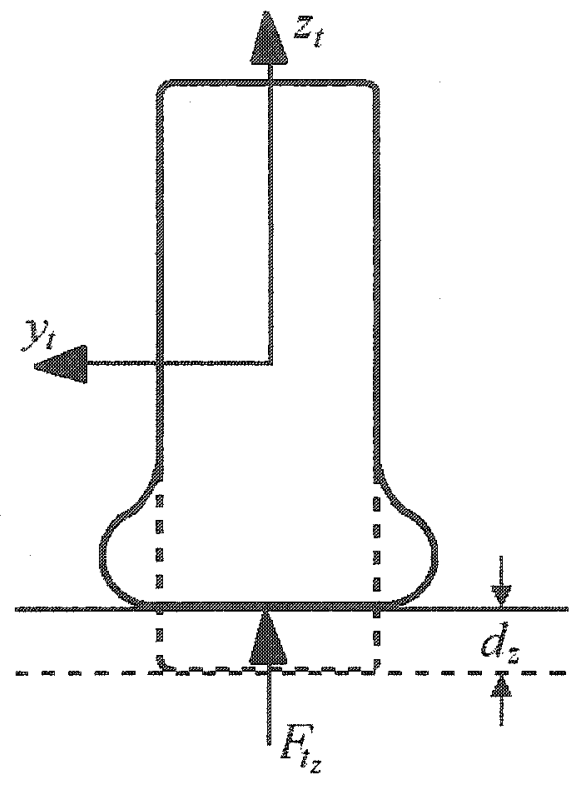

Figure 2.9: Vertical tire deflection

where,

$$
\begin{array}{ll}
f_{1}\left(\frac{d_{z}}{w_{t}}\right)=\frac{0.96\left(\frac{d_{z}}{w_{t}}\right)+0.216\left(\frac{d_{z}}{w_{t}}\right)^{2}}{C_{t z}} & \text { for } \quad \frac{d_{z}}{w_{t}} \leq \frac{10 C_{t z}}{3} \\
f_{1}\left(\frac{d_{z}}{w_{t}}\right)=2.4\left(\frac{d_{z}}{w_{t}}-C_{t z}\right) & \text { for } \quad \frac{d_{z}}{w_{t}}>\frac{10 C_{t z}}{3}
\end{array}
$$

Where $C_{t z}$ is equal to 0.02 for type I tires and 0.03 for types III and VII.

It was found in the development of Dynaface ${ }^{\mathbb{R}}[4]$ that a stiff linear stiffness term had to be added to this relationship for extremely large deflections. This is not required for the planar simulation as it will not encounter extremely large vertical tire deflections in the absence of ship motion.

\section{Lateral Tire Stiffness}

The lateral tire stiffness determines the amount of force that a tire can generate in the lateral direction. The tire must deflect as shown in Figure 2.10 to generate this force. The tire acts like a spring. Again, this parameter remains constant for the planar simulation. 


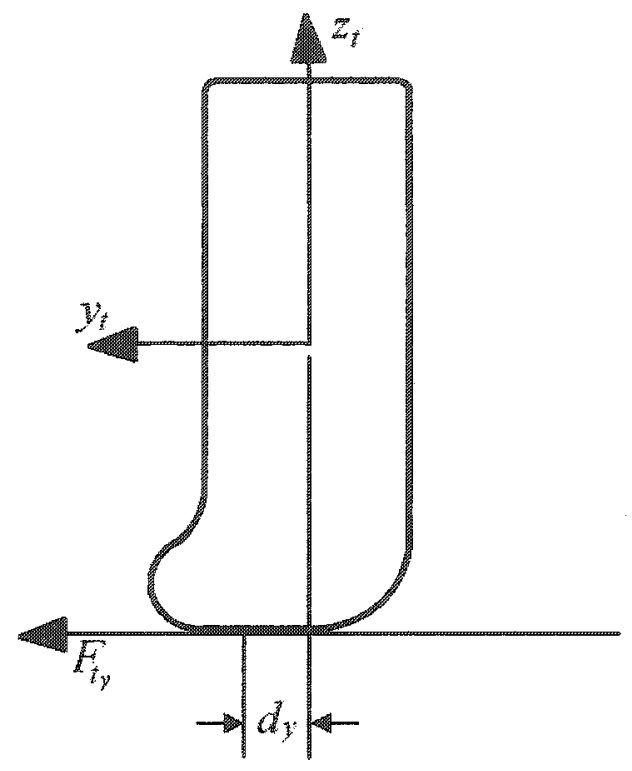

Figure 2.10: Lateral tire deflection

Smiley and Horne [34] have derived an empirical relationship between the vertical tire deflection and the lateral tire stiffness stating as follows:

$$
K_{t_{y}}=\tau w_{t}\left(P+0.24 P_{r}\right)\left[1-\frac{0.7 d_{z}}{w_{t}}\right]
$$

where $\tau$ is equal to 3 for type I tires and 2 for types III and VII.

During the development of Dynaface ${ }^{\circledR}$ [4], it was found that a minimum value for $K_{t y}$ was needed to prevent the lateral tire stiffness from approaching zero in the case of high vertical tire deflection. This minimum value is calculated as follows:

$$
K_{t_{y}}^{m i n}=0.2 \tau w_{t}\left(P+0.24 P_{r}\right)
$$

which limits the lateral tire stiffness to $20 \%$ of the stiffness of a vertically undeflected tire. Again this is not required for the planar simulation which will not experience large vertical tire deflections. 


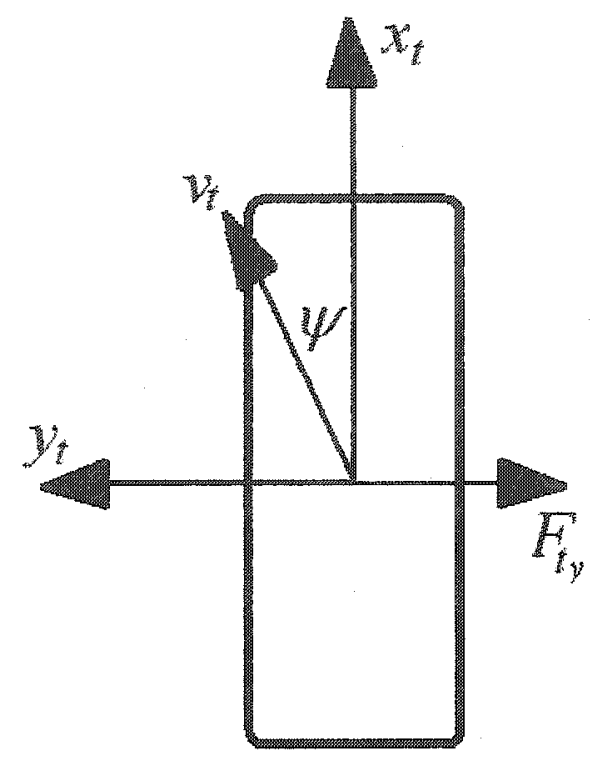

Figure 2.11: The slip defined in the wheels frame of reference

\section{Slip Angle}

Wong [50] defines the slip angle as the angle between the direction the wheel is pointing and the direction it is moving. Figure 2.11 shows a positive slip angle and the direction of the resulting lateral force. The local tire velocity is used to calculate the slip angle, $\psi$. The local tire velocity changes in the planar simulation and therefore the slip angle must be recalculated each time the tire model is used.

\section{Un-Yawed Relaxation Length}

The un-yawed relaxation length is defined as the distance the tire must roll in order for the lateral deformation to drop to a fraction 1/e of its initial value. Smiley and Horne [34] calculate this relaxation length $L_{u}$ using Equation 2.41:

$$
L_{u}=\left(2.8-\frac{0.8 P}{P_{r}}\right)\left(1.0-\frac{4.5 d_{z}}{2 r_{t}}\right) w_{t}
$$


This length is used in Equation 2.48 presented later to calculate the change in lateral tire deflection.

\section{Yawed Relaxation Length}

The yawed relaxation length is defined as the distance the tire must roll in order to generate the lateral tire deflection a fraction 1/e of the difference between its initial value and the steady state value. Smiley and Horne [34] calculate this relaxation length $L_{y}$ using the following equations based on $\frac{d_{z}}{2 r_{t}}$ :

$$
\begin{array}{lrr}
L_{y}=\frac{11 d_{z}}{2 r_{t}}\left(2.8-\frac{0.8 P}{P_{r}}\right) w_{t} & \text { for } & \frac{d_{z}}{2 r_{t}} \leq 0.053 \\
L_{y}=\left(\frac{64 d_{z}}{2 r_{t}}-500\left(\frac{d_{z}}{2 r_{t}}\right)^{2}-1.4045\right)\left(2.8-\frac{0.8 P}{P_{r}}\right) w_{t} & \text { for } & 0.053>\frac{d_{z}}{2 r_{t}}<0.068 \\
L_{y}=\left(0.9075-\frac{4 d_{z}}{2 r_{t}}\right)\left(2.8-\frac{0.8 P}{P_{r}}\right) w_{t} & \text { for } & \frac{d_{z}}{2 r_{t}} \geq 0.068
\end{array}
$$

This length is used in Equation 2.49 presented later to calculate the change in lateral tire deflection.

\section{Cornering Power}

The cornering power represents the amount of lateral force the tire can generate for a given slip angle. It is used to calculate the steady-state lateral force for a given slip angle. Smiley and Horne [34] calculate the cornering power using Equation 2.43.

$$
N=\left(L_{y}+L_{h}\right) K_{t_{y}}
$$

where $L_{h}$ is the half-footprint length, which is a function of the radius and vertical deflection of the tire. Smiley and Horne [34] calculate the half-footprint length using Equa- 
tion 2.44 .

$$
L_{h}=2 r_{t} \sqrt{\left(\frac{d_{z}}{r_{t}}\right)-\left(\frac{d_{z}}{r_{t}}\right)^{2}}
$$

\section{Steady-State Lateral Force}

This is the maximum lateral force that can be developed by a tire for a given slip angle. Following the introduction of a slip angle, a lag will occur before a stead- state lateral force is reached. This steady state lateral force is what is reached as the force is generated by the yawed relaxation. This steady-state lateral force is limited to the value at which skidding would initiate. Smiley and Horne use the cornering power to determine the steady state lateral force as follows:

$$
\begin{array}{lll}
F_{s s_{y}}=\left(\mu F_{t_{z}}^{t}\right)\left(\phi-\frac{4 \phi^{3}}{27}\right) & \text { for } & \phi \leq 1.5 \\
F_{s s_{y}}=\mu F_{t_{z}}^{t} & \text { for } & \phi>1.5
\end{array}
$$

where,

$$
\phi=\frac{N}{\mu F_{t_{z}}^{i}} \psi
$$

\section{Steady-State Lateral Deflection}

The steady-state lateral deflection can be calculated using the lateral tire stiffness as follows:

$$
d_{s s_{y}}=F_{s s_{y}} / K_{t_{y}}
$$

\section{New Lateral Deflection}

Now that all the parameters are determined, an intermediate lateral deflection can be calculated due to the un-yawed or yawed relaxation length. Un-yawed relaxation only 
occurs when the slip angle of the wheel is 0 . This is calculated as follows:

$$
\Delta d_{y}=a_{y}^{i} e^{-\frac{\left|\Delta R_{x y}^{t}\right|}{t_{u}}}
$$

and if the slip angle is not 0 , then yawed relaxation occurs and the change in lateral deflection is calculated using Equation 2.49 based on empirical relationships derived by Smiley and Horne [34].

$$
\Delta d_{y}=d_{s s_{y}}-\left(d_{s s_{y}}-d_{y}^{i}\right) e^{-\frac{\left|\Delta R_{t z}^{t}\right|}{L_{y}}}
$$

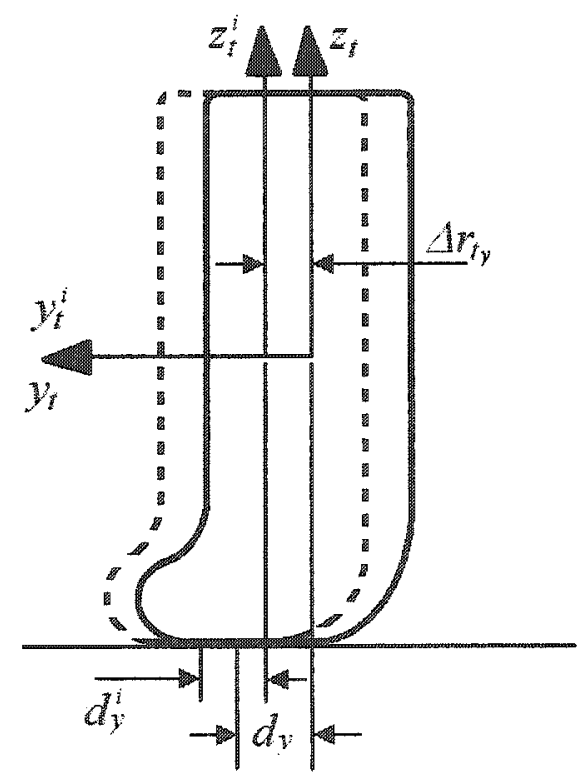

Figure 2.12: Change in lateral tire deflection

Next, the lateral distance the hub travelled must also be considered, as shown in Figure 2.12. This is done as follows:

$$
d_{y}=\Delta d_{y}-\Delta r_{t_{y}}
$$

This allows a lateral tire deflection to be developed without the tire rolling forward. 


\section{Skidding}

Once the now lateral deflection is calculated it has to be tested against the maximum possible frictional force the tire and deck interface can support.

$$
K_{t_{y}} d_{y}>\mu F_{t_{z}}^{t}
$$

If Equation 2.51 is true the tire is skidding and the value of $d_{y}$ must be adjusted so the new lateral force equals the maximum force.

\section{New Lateral Tire Force}

After the new lateral deflection is determined, the overall lateral force can be determined by considering the tire damping as well as the stiffness. This is determined as follows:

$$
F_{t_{y}}^{t}=K_{t_{y}} d_{y}+C_{t_{y}} \Delta V_{t_{y}}^{t}
$$

\section{Rolling Pesistance}

A simple rolling resistance force model is applied in the longitudinal direction of the tire. This force acts in the opposite direction of the wheel's velocity when the wheel has a longitudinal velocity. Currey [51] calculates the rolling resistance force as shown in Equation 2.53

$$
F_{t_{x}}^{t}=\mu_{r r} F_{t_{z}}^{t}\left(1-e^{-\beta\left|V_{t_{x}}^{t}\right|}\right)
$$

It should be noted that the rolling resistance force is not dependent on the velocity of the wheel. A similar smoothing function has been applied to the rolling resistance, as was applied to the Coulomb friction model in Equation 2.26. This prevents a rolling resistance force being applied when the wheel is not moving.

The new lateral and longitudinal tire forces must be transformed into the global frame 


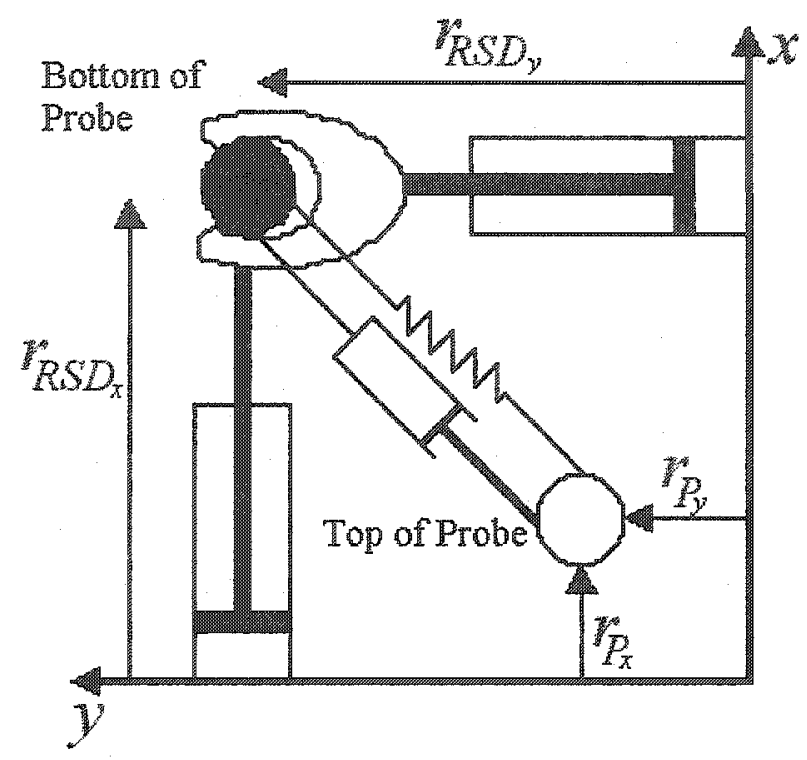

Figure 2.13: Schematic of RSD/probe model showing the spring/damper representation of the probe

of reference and then used to solve the helicopter's equations of motion.

\subsubsection{RSD/Probe Model}

The RSD/probe model interprets input from the control model, described later in Section 2.2 , and generates the forces that should be applied to the helicopter. The deflection in the probe is used to generate the force and the physical operating limitations of the real RSD system are imposed.

The real ASIST RSD is hydraulically powered and controlled using flow-rate valves. By controlling the flow rate, the velocity of the RSD claw is controlled. A hydraulic cylinder representation of the RSD velocities is shown in Figure 2.13. The $\mathrm{x}$ direction is actually a hydraulic winch, but is controlled in the same manner as a cylinder and can be represented in the same manner.

Figure 2.13 also shows the spring/damper representation of the probe that is used to determine the force acting on the probe. Langlois et al [4] use a bilinear spring for 
representing the probe stiffness. This represents the small amount of clearance between the probe and the RSD claw. Therefore, the actual deflection must be compared with a transition deflection. The damping coefficient is also linear, but with only one stage. Equation 2.54 determines the magnitude of the probe force due to the probe stiffness.

$$
\begin{array}{lll}
\left|\vec{F}_{s}\right|=\left|\vec{R}_{R S D / P}\right| K_{P s}^{e f f} & \text { for } & \left|\vec{R}_{R S D / P}\right| \leq D_{P s}^{e f f} \\
\left|\vec{F}_{s}\right|=D_{P s}^{e f f} K_{P s}^{e f f}+\left(\left|\vec{R}_{R S D / P}\right|-D_{P s}^{e f f}\right) K_{P}^{e f f} & \text { for } \quad\left|\vec{R}_{R S D / P}\right|>D_{P s}^{\text {eff }}
\end{array}
$$

where the magnitude and direction of the deflection are determined as follows:

$$
\begin{gathered}
\left|\vec{R}_{R S D / P}\right|=\sqrt{\left(R_{R S D_{x}}^{g}-R_{P_{x}}^{g}\right)^{2}+\left(R_{R S D_{y}}^{g}-R_{P_{y}}^{g}\right)^{2}} \\
\xi_{s}=\arctan \left(\frac{R_{R S D_{y}}^{g}-R_{P_{y}}^{g}}{R_{R S D_{x}}^{g}-R_{P_{x}}^{g}}\right)
\end{gathered}
$$

The direction $\xi_{s}$ is also the direction for the force $\vec{F}_{s}$. The damping component is calculated separately as it may have a different direction than the stiffness component.

$$
\left|\vec{F}_{c}\right|=\left|\vec{R}_{R S D / P}\right| C_{P}^{e f f}
$$

where the magnitude and direction of the rate of change in deflection are determined as follows:

$$
\begin{gathered}
\left|\vec{R}_{R S D / P}\right|=\sqrt{\left(\dot{R}_{R S D_{x}}^{g}-\dot{R}_{P_{x}}^{g}\right)^{2}+\left(\dot{R}_{R S D_{y}}^{g}-\dot{R}_{P_{y}}^{g}\right)^{2}} \\
\xi_{c}=\arctan \left(\frac{\dot{R}_{R S D_{y}}^{g}-\dot{R}_{P_{y}}^{g}}{\dot{R}_{R S D_{x}}^{g}-\dot{R}_{P_{x}}^{g}}\right)
\end{gathered}
$$

Again the direction $\xi_{c}$ is also the direction of the force $\vec{F}_{c}$. The two forces are combined in 


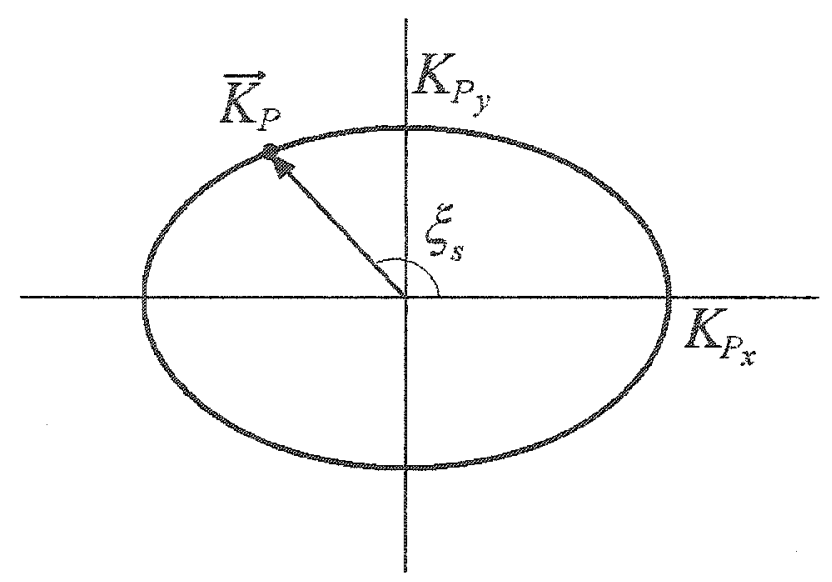

Figure 2.14: Stiffness ellipse for the large deflection stiffness $\left(K_{P}^{e f f}\right)$

Equation 2.60 to obtain the total force acting on the probe in the global frame of reference.

$$
\vec{F}_{P}^{g}=\vec{F}_{s}^{g}+\vec{F}_{c}^{g}
$$

A stiffness and damping ellipse, Figure 2.14 for the large deflection stiffness $\left(K_{P}^{e f f}\right)$, has been applied to allow for different values in the global $\mathrm{x}$ and $\mathrm{y}$ directions. The direction of the probe deflection and velocity determines the effective stiffness and damping values. The magnitude of the vector (in the case of Figure 2.14 this is $\left(\vec{K}_{P}\right)$ ) is used to determine the effective value. These values are determined as follows:

$$
\begin{aligned}
& K_{P}^{e f f}=\frac{1}{\sqrt{\left(\frac{\cos ^{2} \xi_{s}}{K_{P_{x}}}\right)^{2}+\left(\frac{\sin ^{2} \xi_{s}}{K_{P_{y}}}\right)^{2}}} \\
& K_{P s}^{e f f}=\frac{1}{\sqrt{\left(\frac{\cos ^{2} \xi_{s}}{K_{P_{s}}}\right)^{2}+\left(\frac{\sin ^{2} \xi_{s}}{K_{P_{s_{y}}}}\right)^{2}}} \\
& D_{P s}^{e f f}=\frac{1}{\sqrt{\left(\frac{\cos ^{2} \xi_{s}}{D_{P s_{x}}}\right)^{2}+\left(\frac{\sin ^{2} \xi_{s}}{D_{P_{s}}}\right)^{2}}}
\end{aligned}
$$


and the effective damping coefficient is:

$$
C_{P}^{e f f}=\frac{1}{\sqrt{\left(\frac{\cos ^{2} \xi_{c}}{C_{P_{x}}}\right)^{2}+\left(\frac{\sin ^{2} \xi_{c}}{C_{P_{y}}}\right)^{2}}}
$$

Once the probe force has been calculated, it is tested against the maximum RSD force and if it is above the limiting force, the probe deflections are adjusted for the maximum force. Similar corrections are made with the RSD position limits. These limitations restrict the position of the RSD and in effect the helicopter along with the forces that the RSD is able to apply to the probe. The maximum force the RSD can produce is determined by the pressure in the hydraulic system.

\subsubsection{Aerodynamic Model}

A simple aerodynamic drag model is used to simulate the wind forces acting on the helicopter. The general equation for determining the drag force is described by White [52] as Equation 2.65 .

$$
F_{d}=\frac{1}{2} \rho V_{w}^{2} C_{D} A
$$

The characteristic area, $A$, is the profile area of the object perpendicular to the wind velocity. The drag coefficient, $C_{D}$, is based on the overall shape of the object. Dynaface ${ }^{(B)}[4]$ uses this method, but lumps $C_{D}$ and $A$ together, calling it the equivalent area. Equivalent areas are usually obtained by wind tunnel testing.

The drag forces are calculated in the longitudinal direction using Equation 2.66 and the lateral direction using Equation 2.67, and applied at the centre of pressure for each. The position of the centre of pressure can be different for each direction, so all calculations 
are performed in the helicopter frame of reference.

$$
\begin{aligned}
& F_{d x}^{h}=\frac{1}{2} \rho V_{w x}^{h}\left|V_{w x}^{h}\right| A_{e x} \\
& F_{d y}^{h}=\frac{1}{2} \rho V_{w y}^{h}\left|V_{w y}^{h}\right| A_{e y}
\end{aligned}
$$

Only the wind velocity and/or ship velocity is considered when determining the drag forces. The manoeuvring speed is insignificant compared with the apparent wind speed when significant aerodynamic forces occur, and can be neglected.

\subsection{Control Model}

The control model allows for three different methods of controlling the HeliMan simulation: tabulated input values can be read as the simulation is running; autonomous controller algorithms can be incorporated and evaluated; and an interactive joystick can be used to allow the user to manoeuvre the helicopter in realtime using a simple graphical interface. Details on how to control the RSD using each control option are available in Appendix A.

\subsubsection{Joystick Input}

The joystick input option mimics the joystick controller used in the real ASIST system. A joystick position is used to specify a velocity to the RSD/Probe model. Figure 2.15 shows the velocity input profiles that the joystick applies based on its position. The $Y_{J}$ axis specifies the lateral RSD direction and the $X_{J}$ axis specifies the longitudinal RSD direction. The lateral RSD direction is controlled by a simple on/off step function, while the longitudinal direction is controlled by a ramp function. Both directions also have a specific amount of dead band travel.

This joystick axis configuration is dependent on where the control panel is situated 


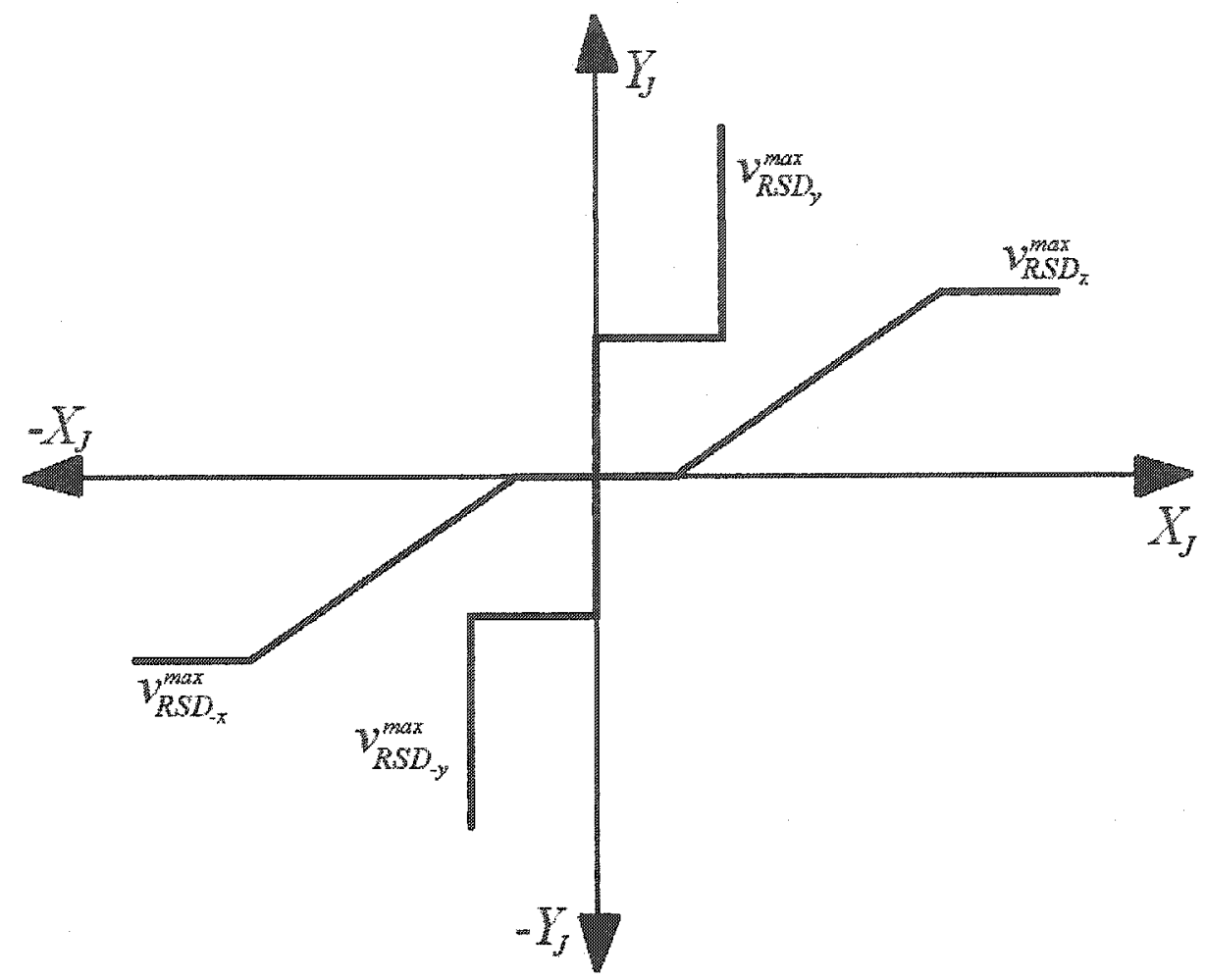

Figure 2.15: Graph showing how the joystick specifies the RSD velocity

with respect to the flight deck. The configuration shown in Figure 2.15 is with the control panel starboard of the flight deck. If the control panel is forward of the flight deck, the joystick axes would have to be rotated $90^{\circ}$ in the positive direction while freezing the velocity profiles.

Two button functions are also available on the joystick. The first button models the float command. With the ASIST system, the operator has the option of letting the RSD float in a particular direction while it is moved in the direction perpendicular to the floating direction. This essentially makes the applied force at the probe zero in the floating direction by activating a pressure release valve. If the float button is activated, the simulation relaxes the probe deflection by moving the RSD claw in the floating direction and causing the force acting on the probe to go to zero. Equations 2.68 and 2.69 demonstrates how the lateral float is implemented in the joystick model. A lag coefficient, $\gamma_{l a g}$ prevents the floating 
process from occurring instantly.

$$
\begin{gathered}
\text { new } \dot{R}_{R S D_{y}}^{g}=\dot{R}_{P_{y}}^{g} \\
\operatorname{new} R_{R S D_{y}}^{g}=R_{R S D_{y}}^{g}-\gamma_{l a g}\left(R_{R S D_{y}}^{g}-R_{P_{y}}^{g}\right)
\end{gathered}
$$

The second button specifies a steering angle option for the castor wheel in the simulation. Some helicopter types have steerable nose wheels that can be locked at specific steering angles. These predetermined steering angles are made available to the simulation in an input file. The button toggles through the steering angle options along with the free castor wheel option. Refer to Appendix A for more detail on how to specify these angles.

A graphics option is available to provide the HeliMan operator feedback while controlling the RSD with the joystick. Figure 2.16 is a screen shot of the graphical option available in HeliMan. Figure 2.17 is a labelled version of Figure 2.16. The graphical option can be used with all of the control options to visually verify what the simulation is doing.

\subsubsection{File Input}

If selected, HeliMan reads the input values from a file that has been generated using experimental results, hand calculations, or using HeliMan itself. In the latter case, input values are recorded in a file while the simulation is run in realtime using the joystick. The simulation run can then be repeated using this file of input values. This feature is useful during validation and for analysis where repeating inputs exactly, which would be very difficult with a joystick, is necessary.

The first method of file input uses the RSD velocities specified by the joystick and applies them the same manner as direct joystick commands. The button functions are also applied using the file input method. Button inputs can also be ignored by setting 


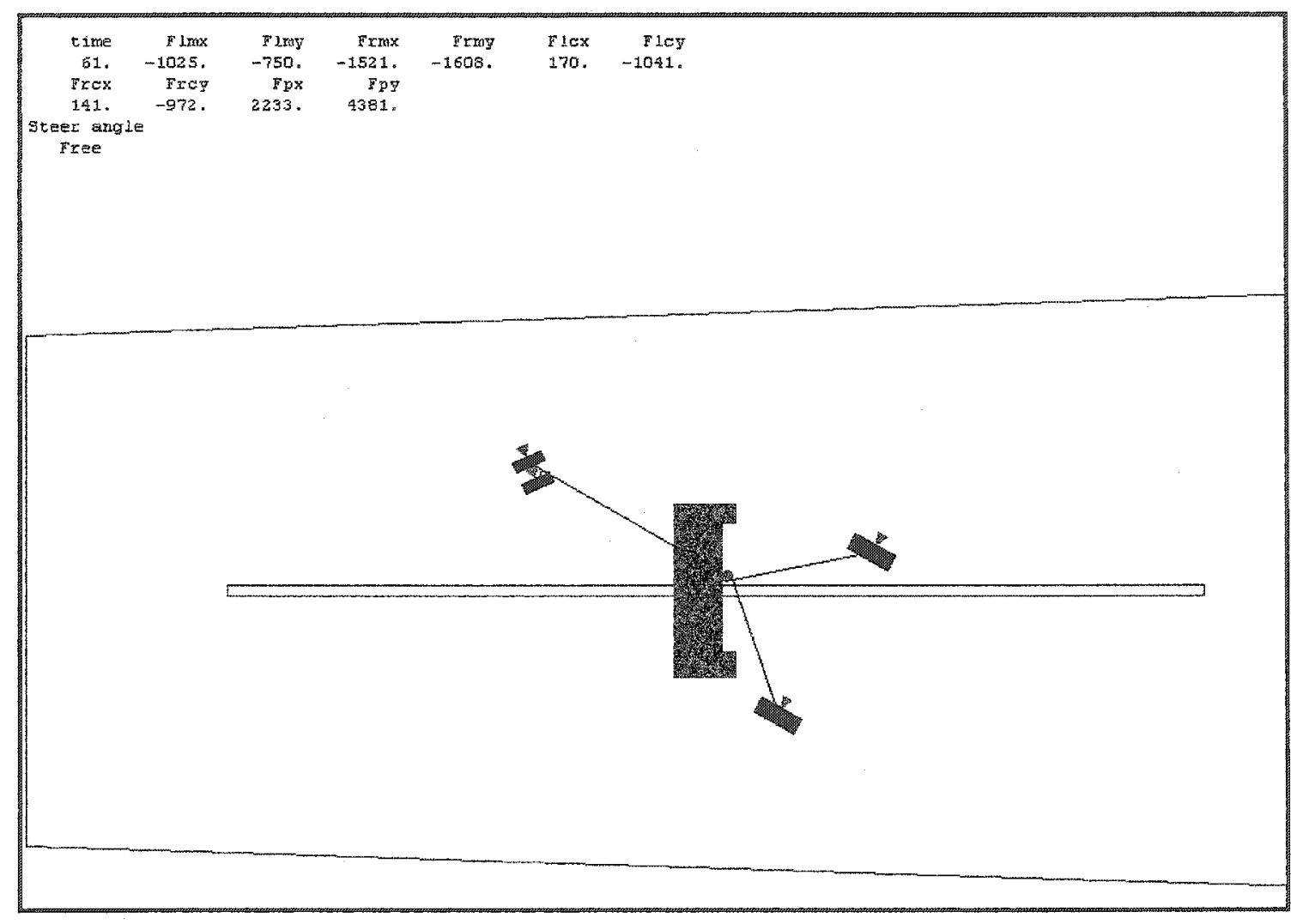

Figure 2.16: Heliman screen shot 


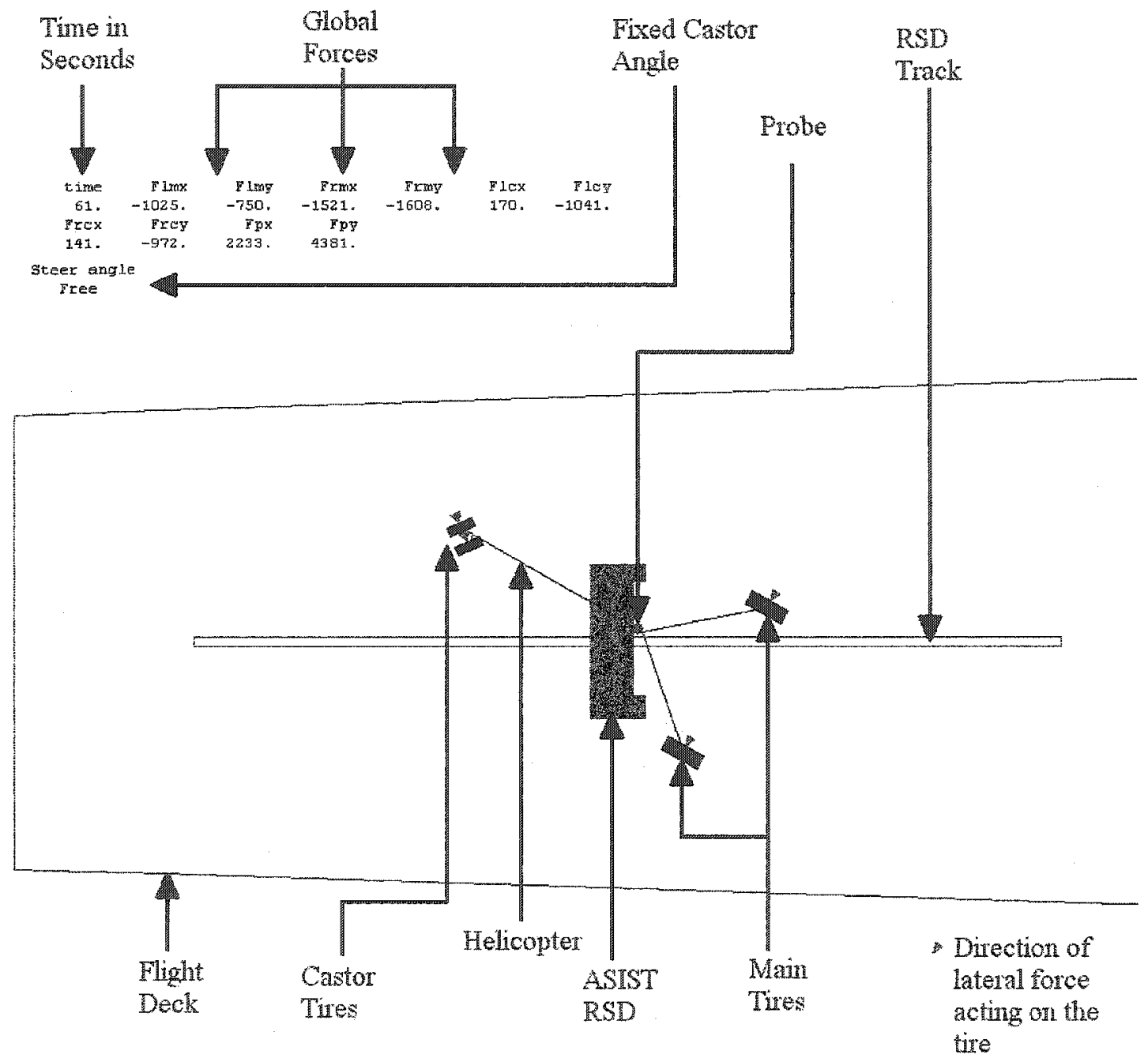

Figure 2.17: Labelled HeliMan screen shot 
them to zero in the input file. Heliman has also been configured to run without the RSD/Probe model being used, by applying force profiles specified in the input file directly to the helicopter.

\subsubsection{Controller Input}

The controller input uses parameters in HeliMan to determine an ideal manoeuvring trajectory for the helicopter. The helicopter can then be automatically straightened without the need of an operator. This control algorithm option will determine and specify RSD velocities using classical control methods. The nonlinear nature of the system does not lend itself to traditional transfer function representation. Therefore, controller development must be done in the time domain using the simulation as the plant. The development of an automated control algorithm is described by Feldman [53].

\subsection{Simulation Model}

The simulation model combines the parts described in Sections 2.1 and 2.2. These parts include the equations of motion, tire model, RSD/probe model, castor joint model, aerodynamic model, and control model. Figure 2.18 illustrates how these parts are applied to the simulation process. All of the physical helicopter properties are input, along with the simulation parameters, which include the simulation time, initial position, and control profiles (if the joystick is not being used).

Next, the initial forces and displacements are calculated based on the initial conditions specified by the user. Also at this point all position vectors are transformed into the helicopters frame of reference from the manufacturer's frame. The static load distribution on the tires is determined based on the specified aircraft weight and then used to calculate the vertical displacement of each tire. These vertical tire deflections are kept constant for 
the duration of the simulation run.

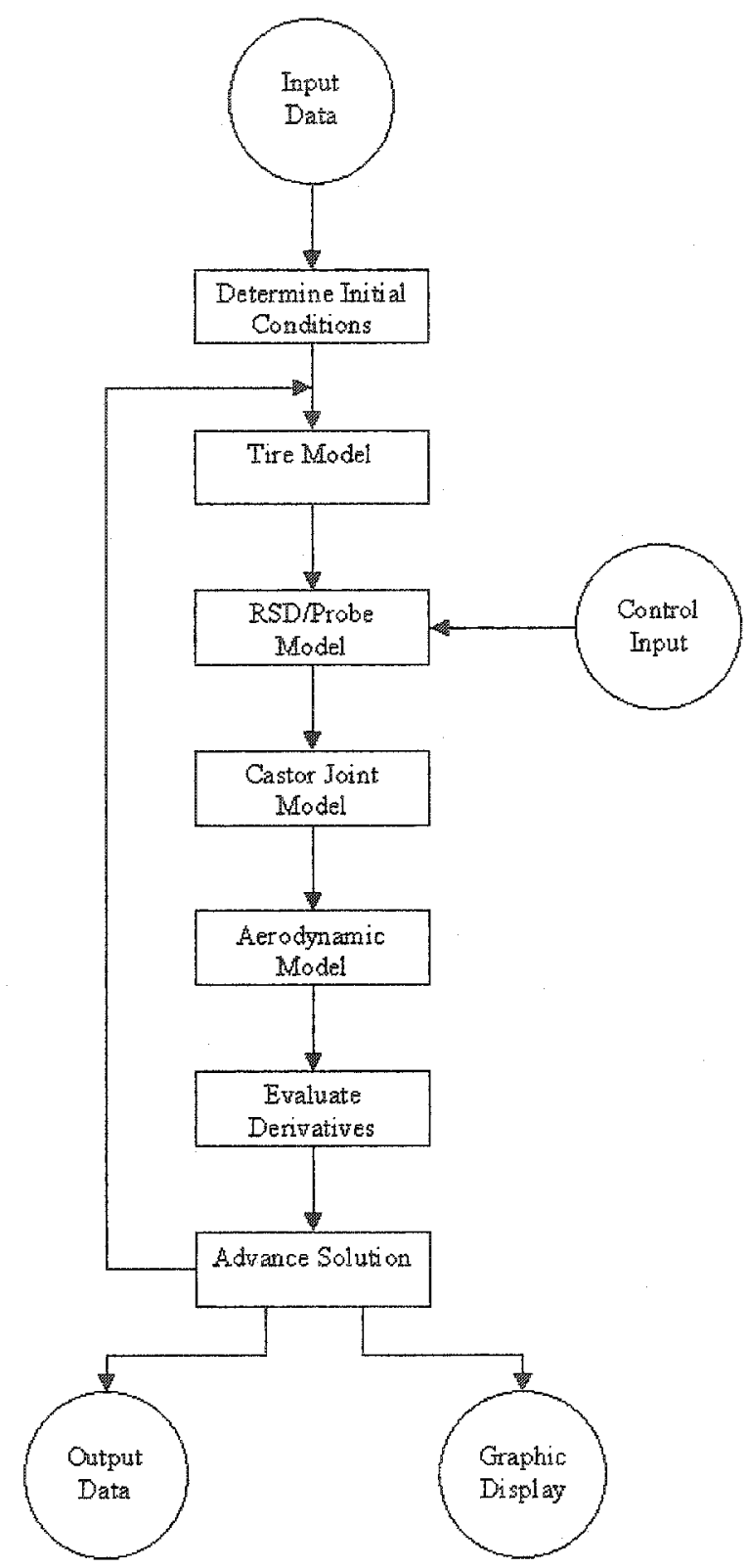

Figure 2.18: Flow chart of simulation

Once the initial conditions have been setup, the numerical integrator drives the solution forward in time, while obtaining control inputs from the joystick, input file, or internal calculations. The numerical integrator needs to be both accurate as well as fast enough to drive the simulation in real time. A fixed-time step fourth-order Runge-Kutta method 
has been found to meet numerical integration requirements. Section 2.3.1 describes this form of numerical integration in greater detail.

As the simulation runs, information is gathered and output to a file for subsequent postprocessing and analysis. This information not only includes the output from the various models, but also can include an input file in the form of joystick inputs or probe forces. These input files allow a simulation being controlled by a joystick to be repeated while adjustments to the simulation can be made, as described previously.

\subsubsection{Numerical Methods}

Two common numerical methods have been implemented in HeliMan to propagate the solution in time. A fourth-order Runge-Kutta numerical integrator is implemented to solve the differential equations and Gaussian elimination is used to solve for the unknown accelerations and forces at each time step based on Equation 2.24. An analytical solution was determined in Appendix A, but was found to be impractical for implementation. The complete numerical solution has been found to run faster than realtime for this application, with the simulation running at 10000 cycles per second and displaying the graphics at 24 frames per second on an $800 \mathrm{MHz}$ computer.

\section{Fourth-Order Runge-Kutta Method}

To propagate the solution, the classical fourth-order Runge-Kutta method has been implemented. Chapra and Canale [54] present this popular numerical integration method with the general solution at time step $j+1$ as:

$$
y_{j+1}=y_{j}+\frac{h}{6}\left(k_{1}+2 k_{2}+2 k_{3}+k_{4}\right)
$$


where,

$$
\begin{aligned}
& k_{1}=f\left(t_{j}, y_{j}\right) \\
& k_{2}=f\left(t_{j}+\frac{h}{2}, y_{j}+\frac{h}{2} k_{1}\right) \\
& k_{3}=f\left(t_{j}+\frac{h}{2}, y_{j}+\frac{h}{2} k_{2}\right) \\
& k_{4}=f\left(t_{j}+h, y_{j}+h k_{3}\right)
\end{aligned}
$$

The fourth-order Runge-Kutta method makes three estimates of the solution over a given time step. The slope of the previous point is used to determine the next one in the order shown above. Two are taken at half the time step and one over the full time step with more weight given to the values determined at the half time step.

\section{Gaussian Elimination with Partial Pivoting}

To solve the set of simultaneous linear equations represented as the matrix Equation 2.24 at each time step, Gaussian elimination with partial pivoting has been implemented. Chapra and Canale [54] demonstrate Gauss elimination for three simultaneous equations in the form $[a]\{x\}=\{c\}$, as follows:

$$
\left[\begin{array}{lllll}
a_{11} & a_{12} & a_{13} & \mid & c_{1} \\
a_{21} & a_{22} & a_{23} & \mid & c_{2} \\
a_{31} & a_{32} & a_{33} & \mid & c_{3}
\end{array}\right] \Rightarrow\left[\begin{array}{ccccc}
a_{11} & a_{12} & a_{13} & \mid & c_{1} \\
& a_{22}^{\prime} & a_{23}^{\prime} & \mid & c_{2}^{\prime} \\
& a_{33}^{\prime \prime} & \mid & c_{3}^{\prime \prime}
\end{array}\right] \Rightarrow \begin{gathered}
x_{3}=c_{3}^{\prime \prime} / a_{33}^{\prime \prime} \\
x_{2}=\left(c_{2}^{\prime}-a_{23}^{\prime} x_{3}\right) / a_{22}^{\prime} \\
x_{1}=\left(c_{1}-a_{12} x_{1}-a_{13} x_{3}\right) / a_{11}
\end{gathered}
$$

Matrix $[a]$ is augmented with vector $\{c\}$. Elementary row and column operations are performed to put the augmented matrix into the upper triangular form. The vector $\{x\}$ can then be solved from the bottom up. Partial pivoting involves reordering the equations to ensure larger values occur on the diagonal of matrix [a]. This increases the accuracy of the solution. More sophisticated methods are available to solve the linear equations, but the Gaussian elimination approach was found to be adequate in this application. 


\subsubsection{Transformation Matrices}

All of the different forces that need to be evaluated are determined in the most appropriate coordinate systems. An example of this are the tire forces that are calculated in the tire coordinates, but must be transformed into global coordinates to solve Equation 2.24. The following planar transformation matrices shown in Equations 2.71 and 2.72 have been used for all rotational transformations.

$$
\begin{aligned}
& {\left[T^{l \rightarrow g}\right]=\left[\begin{array}{cc}
\cos \theta & -\sin \theta \\
\sin \theta & \cos \theta
\end{array}\right]} \\
& {\left[T^{g \rightarrow l}\right]=\left[\begin{array}{cc}
\cos \theta & \sin \theta \\
-\sin \theta & \cos \theta
\end{array}\right]}
\end{aligned}
$$

where $\theta$ is $q_{3}, q_{4}$ or $q_{3}+q_{4}$ and $g$ and $l$ refer to global and local coordinates respectively. Refer to Figure 2.1 to see the different coordinate systems used in the equation derivation. 


\section{Chapter 3}

\section{Verification and Validation}

The process of scrutinizing the planar simulation, HeliMan, has been broken down into two stages: verification and validation. The verification stage was conducted to make sure that the mathematical model has been implemented correctly in the simulation. The validation stage used experimental data to test how well the mathematical model represents the nonlinear system being simulated. First, the verification stage is presented in the following section.

\subsection{Verification}

The verification process was carried out to ensure that the mathematical model presented in Chapter 2 has been implemented correctly in HeliMan. Particular emphasis has been placed on the helicopter dynamics model. The joystick control model was also verified. This was done by breaking down the helicopter dynamics model into functions in the same manner as they were presented in Section 2.1. This breakdown was as follows:

- equations of motion;

- castor joint model;

- tire model; 
- RSD/probe model;

- aerodynamic model; and

- joystick control model.

For each function the initial conditions of the simulation are described. The initial conditions entered into HeliMan are slightly different for each function to help emphasize the particular function being verified. A range of results are generated using the joystick to test important aspects of the function. The inputs and outputs from the particular function being tested in HeliMan are recorded. Then at a particular point in simulation time, the inputs were used to generate hand calculation results for comparison with those generated by HeliMan. These points were not randomly chosen, but were chosen to test the limits of the function being tested. These limits represent the physical properties of the model such as the maximum force the RSD claw can apply to the probe.

With the exception of the helicopter's equations of motion, these models do not contain dynamics, but rather a series of equations that are exercised consistently at every time step. Consequently, verification at key points in the simulation is sufficient to ensure the models have been implemented correctly.

Verification and validation was performed using parameter values that are representative of a Sikorsky SH-60B Seahawk helicopter. As stated in Chapter 1, this choice is based on the availability of a DLTV on ITI's ITF, which is based on the Seahawk. The DLTV landing gear is configured with a castoring tail wheel assembly with dual tires and single main tires. The results of the verification process are presented next.

\subsubsection{Equations of Motion Comparison}

The verification results for the equations of motion are presented in Table 3.1. These values were obtained by solving Equation 2.24 for a given set of inputs at a given point 
in time. The values calculated are the four state accelerations, $\ddot{q}_{1}$ through $\ddot{q}_{4}$, and the reaction forces at the castor joint, $F_{B_{x}}^{g}$ and $F_{B_{y}}^{g}$.

This test was performed for four distinct situations starting with the helicopter and castor in an aligned orientation:

1. moving laterally to port;

2. moving laterally to starboard;

3. moving longitudinally forward; and

4. swinging the tail out to starboard while traversing forward.

The number of each situation corresponds to a calculation point in Table 3.1. The percent difference for each calculation has been found to be zero meaning the equations of motion have been implemented correctly in HeliMan for up to eight significant figures.

Table 3.1: Verification results for the equations of motion

\begin{tabular}{|c|c|c|c|c|c|}
\hline & \multirow[b]{2}{*}{ Method } & \multicolumn{4}{|c|}{ Calculation Points } \\
\hline & & 1 & 2 & 3 & 4 \\
\hline \multirow{3}{*}{$\begin{array}{c}\ddot{q}_{1} \\
\left(\mathrm{ft} / \mathrm{s}^{2}\right)\end{array}$} & HeliMan & 0.02889383 & 0.01507871 & 0.00029003 & -0.45964637 \\
\hline & Hand & 02889383 & 0.01507871 & 0.00029003 & -0.45964638 \\
\hline & $\%$ diff & $\overline{0}$ & 0 & 0 & $\sim 0$ \\
\hline \multirow{3}{*}{$\begin{array}{c}\ddot{q}_{2} \\
\left(\mathrm{ft} / \mathrm{s}^{2}\right)\end{array}$} & HeliMan & .20814987 & -0.08157589 & 0.00000028 & 0.14676765 \\
\hline & Hand & -0.20814987 & -0.08157589 & 0.00000028 & 0.14676765 \\
\hline & $\%$ diff & 0 & 0 & 0 & 0 \\
\hline \multirow{3}{*}{$\begin{array}{c}\ddot{q}_{3} \\
\left(\mathrm{rad} / \mathrm{s}^{2}\right)\end{array}$} & HeliMan & 0.00601088 & 0.01311537 & -0.00000050 & -0.04080768 \\
\hline & Hand & 0.00601088 & 0.01311537 & -0.00000050 & -0.04080768 \\
\hline & $\%$ diff & 0 & 0 & 0 & 0 \\
\hline \multirow{3}{*}{$\begin{array}{c}\ddot{q}_{4} \\
\left(\mathrm{rad} / \mathrm{s}^{2}\right)\end{array}$} & HeliMan & .16720881 & -0.13082611 & -0.00000715 & 0.12028903 \\
\hline & Hand & -0.16720881 & -0.13082611 & -0.00000715 & 0.12028902 \\
\hline & \% diff & 0 & 0 & 0 & $\sim 0$ \\
\hline \multirow{3}{*}{$\begin{array}{c}F_{B_{x}}^{g} \\
\text { (lbs) }\end{array}$} & HeliMan & 1699.6229 & 1989.5976 & -356.9803 & -367.63254 \\
\hline & Hand & 1699.6229 & 1989.5976 & -356.9803 & -367.63254 \\
\hline & $\%$ & 0 & 0 & 0 & 0 \\
\hline \multirow{3}{*}{$\begin{array}{c}F_{B_{y}}^{g} \\
(1 \mathrm{bs})\end{array}$} & HeliMan & -1952.1970 & 1792.7420 & -0.01428072 & 19.009091 \\
\hline & & -1952.1970 & 1792.7420 & -0.01428072 & 19.009091 \\
\hline & $\%$ diff & 0 & 0 & 0 & 0 \\
\hline
\end{tabular}




\subsubsection{Castor Joint Model Comparison}

Next, the verification results for the castor joint model are presented in Table 3.2. These values were obtained by solving Equations 2.25 through 2.32 developed for the castor joint model. The values calculated were the torque acting on the castor joint, $M_{B}$, and the kingpin inclination force acting on each castor wheel, $F_{\kappa_{1}}$ and $F_{\kappa_{2}}$. It is important to note that the Coulomb friction was calculated using the reaction forces at the castor joint calculated during the previous time step (typically less than $1 \mathrm{~ms}$ earlier depending on the fixed time step chosen).

This test was performed for two points starting with the helicopter and castor in an aligned orientation:

1. moving laterally to port; and

2. moving laterally to starboard.

The number of each situation corresponds to a calculation point in Table 3.2. The percent difference for each calculation has been found to be zero, therefore the castor joint model has been implemented correctly in HeliMan, thereby producing correct results up to eight significant figures.

Table 3.2: Verification results for the castor joint model

\begin{tabular}{|c|c|c|c|}
\hline & \multirow[b]{2}{*}{ Method } & \multicolumn{2}{|c|}{ Calculation Points } \\
\hline & & 1 & 2 \\
\hline \multirow{3}{*}{$\begin{array}{c}M_{B} \\
(\mathrm{ft} \cdot \mathrm{lbs})\end{array}$} & HeliMan & 601.13954 & -710.19530 \\
\hline & Hand & 601.13954 & -710.19530 \\
\hline & $\%$ diff & 0 & 0 \\
\hline \multirow{3}{*}{$\begin{array}{c}F_{\kappa_{1}} \\
(\mathrm{lbs})\end{array}$} & HeliMan & 180.46651 & -306.36895 \\
\hline & Hand & 180.46651 & -306.36895 \\
\hline & $\%$ diff & 0 & 0 \\
\hline \multirow{3}{*}{$\begin{array}{c}F_{\kappa_{2}} \\
(\mathrm{lbs})\end{array}$} & HeliMan & 180.46651 & -306.36895 \\
\hline & Hand & 180.46651 & -306.36895 \\
\hline & $\%$ diff & 0 & 0 \\
\hline
\end{tabular}




\subsubsection{Tire Model Comparison}

The tire model has been implemented in two parts in HeliMan. The first part calculates the force acting on the given tire inside the integrator. Outside the integrator the second part calculates the change in lateral tire defiection for the given tire. This was done to help speed up the simulation without compromising accuracy, by minimizing the calculations that need to be made during each time step.

First the verification results for the tire force model are presented in Table 3.3. These values were obtained by solving Equations 2.52 and 2.53 proposed for the tire model. The values calculated were the longitudinal and lateral forces acting on the tire, $F_{t_{x}}^{t}$ and $F_{t_{y}}^{t}$, respectively. The vertical force, $F_{t_{z}}^{t}$ does not change for the planar simulation.

This test was performed on two different tire types during four distinct situations starting with the helicopter and castor in an aligned orientation:

1. left main tire moving laterally to starboard;

2. left main tire moving laterally to port;

3. right castor tire moving laterally to port; and

4. right castor tire moving laterally to starboard.

The number of each situation corresponds to a calculation point in Table 3.3. The percent difference for each calculation has been found to be zero, therefore the tire force model has been implemented correctly in HeliMan.

The verification results for the tire deflection model are presented in Table 3.4. These values were obtained by solving Equations 2.41 through 2.50 proposed for the tire model. The value being calculated was the new lateral tire deflection, $d_{y}$, of the tire. The old lateral tire deflection is also presented in Table 3.4 to demonstrate how it changes. The changes in the tire deflection are small due to the small time step needed to perform the fourth-order Runge-Kutta integration accurately. 
Table 3.3: Verification results for the tire force model

\begin{tabular}{|c|c|c|c|c|c|}
\hline & \multirow{4}{*}{} & \multicolumn{4}{|c|}{ Calculation Points } \\
\cline { 2 - 6 } & Method & 1 & 2 & 3 & 4 \\
\hline \multirow{2}{*}{$F_{t_{x}}^{t}$ (lbs) } & HeliMan & 352.99383 & -365.69949 & -178.48908 & -178.48908 \\
\cline { 2 - 6 } & Hand & 352.99384 & -365.69949 & -178.48908 & -178.48908 \\
\cline { 2 - 6 } & \% diff & $\sim 0$ & 0 & 0 & 0 \\
\hline \multirow{2}{*}{$F_{t_{y}}^{t}$} & HeliMan & 387.97075 & -924.84179 & -1107.02488 & 923.39625 \\
\cline { 2 - 6 }$(\mathrm{lbs})$ & Hand & 387.97075 & -924.84180 & -1107.02487 & 923.39625 \\
\cline { 2 - 6 } & $\%$ diff & 0 & $\sim 0$ & $\sim 0$ & 0 \\
\hline
\end{tabular}

This test was performed on two different tires for five distinct situations starting with the helicopter and castor in an aligned orientation:

1. right main tire moving laterally to port;

2. right main tire moving laterally to starboard;

3. right main tire traversing forward with an initial tire deflection;

4. left castor tire moving laterally to port; and

5. left castor tire moving laterally to starboard.

The number of each situation corresponds to a calculation point in Table 3.4. The percent difference for each calculation has been found to be zero, therefore the tire deflection model has been implemented correctly in HeliMan.

Table 3.4: Verification results for the tire deflection model

\begin{tabular}{|c|c|c|c|c|c|c|}
\hline & & \multicolumn{5}{|c|}{ Calculation Points } \\
\cline { 3 - 7 } & Method & 1 & 2 & 3 & 4 & 5 \\
\hline old $d_{y}$ & Input & -0.00053810 & 0.03649234 & 0.09553110 & -0.07676869 & 0.13169686 \\
\hline \multirow{2}{*}{$\begin{array}{c}\text { new } d_{y} \\
\text { (ft) }\end{array}$} & HeliMan & -0.00053801 & 0.03648070 & 0.09552347 & -0.07677881 & 0.13171379 \\
\cline { 2 - 7 } & Hand & -0.00053801 & 0.03648070 & 0.09552347 & -0.07677881 & 0.13171379 \\
\cline { 2 - 7 } & $\%$ diff & 0 & 0 & 0 & 0 & 0 \\
\hline
\end{tabular}

Calculation point three is a good example of how the tire model is able to relax lateral deflections when rolling longitudinally. For calculation point three, the left and right main 
tires were given opposite initial tire deflections of 1.2 inches. Table 3.4 only shows a single step calculation where the lateral tire deflection can be observed relaxing in the right main tire beyond the fourth decimal place. Figure 3.1 shows how both the left and right main tires relax as the helicopter was traversed.

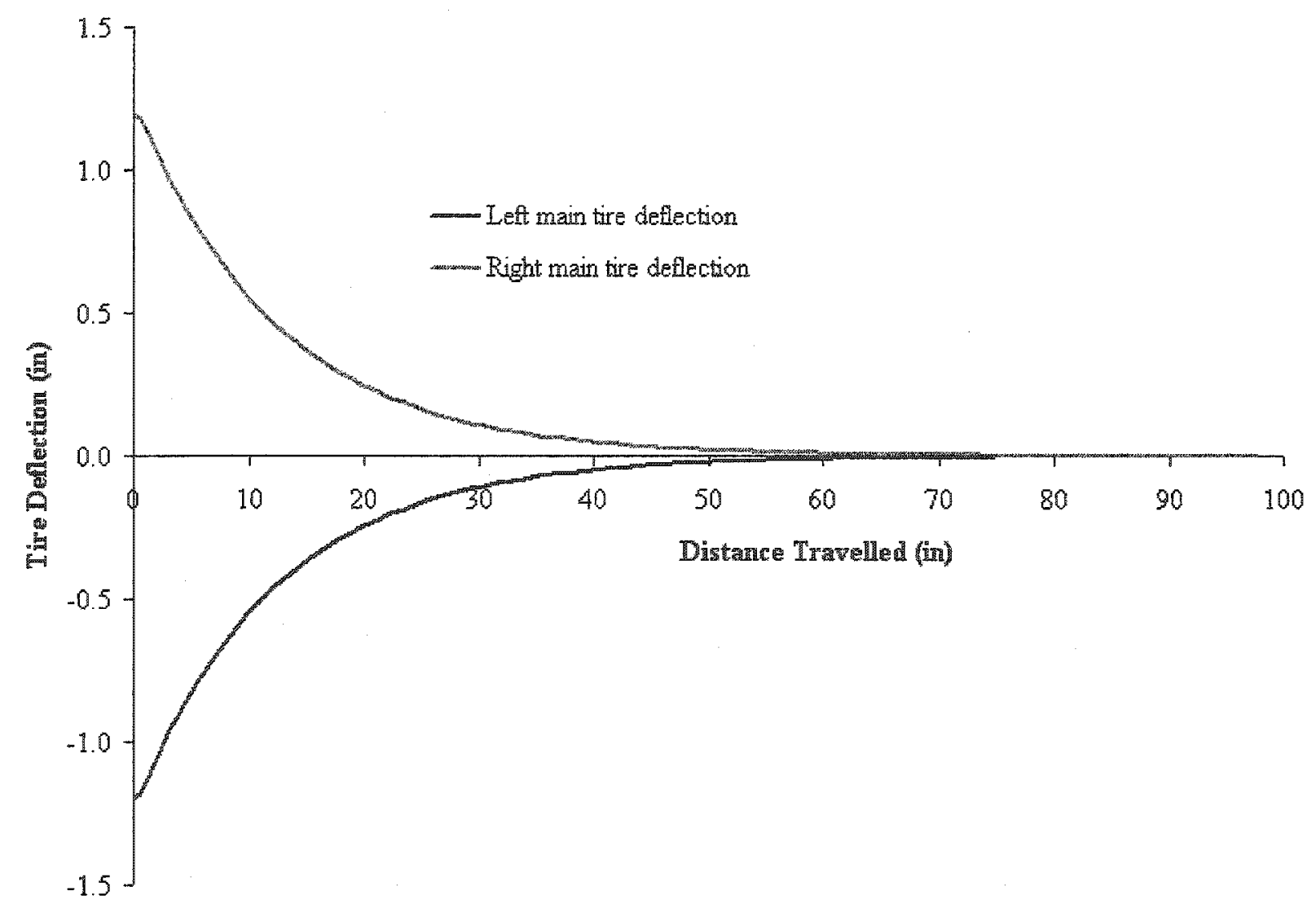

Figure 3.1: Demonstration of the relaxation of tire deflections in the main tires

\subsubsection{RSD/probe Model Comparison}

The verification results for the RSD/probe model are presented in Table 3.5. These values were obtained by solving Equations 2.54 through 2.64 developed for the RSD/probe model. The values calculated are the new probe deflection components, $R_{R S D / P_{x}}$ and $R_{R S D / P_{y}}$ and the corresponding probe force components, $F_{P_{x}}^{g}$ and $F_{P_{y}}^{g}$. 
This test was performed for four distinct situations starting with the helicopter and castor in an aligned orientation:

1. maximum lateral RSD force to port;

2. longitudinal RSD force forward;

3. maximum lateral RSD force to starboard; and

4. maximum longitudinal RSD force forward with unrestricted side load.

The number of each situation corresponds to a calculation point in Table 3.5. The percent difference for each calculation has been found to be zero, therefore the tire deflection model has been implemented correctly in HeliMan.

Table 3.5: Verification results for the RSD/probe model

\begin{tabular}{|c|c|c|c|c|c|}
\hline & \multirow[b]{2}{*}{ Method } & \multicolumn{4}{|c|}{ Calculation Points } \\
\hline & & 1 & 2 & 3 & 4 \\
\hline old $R_{R S D / P_{x}}$ & Input & -0.00839958 & 0.04733692 & -0.01220765 & 0.05667466 \\
\hline \multirow{3}{*}{$\begin{array}{c}\text { new } R_{R S D / P_{x}} \\
(\mathrm{ft})\end{array}$} & HeliMan & -0.00840525 & 0.04733692 & -0.01221087 & 0.05667466 \\
\hline & Hand & 0840 & 0.04733 & -0.01221 & $\overline{05667466}$ \\
\hline & $\%$ diff & 0 & $\overline{0}$ & $\overline{0}$ & 0 \\
\hline old $\bar{R}_{R}$ & Input & $\overline{05774714}$ & 0.00769550 & -0.05753257 & -0.02777371 \\
\hline \multirow{3}{*}{$\begin{array}{c}\text { new } R_{R S D / P_{y}} \\
(\mathrm{ft})\end{array}$} & HeliMan & 0.05770357 & 0.00769550 & -0.05751489 & -0.02777371 \\
\hline & Hand & 0.05770357 & 0.00769550 & -0.05751489 & -0.02777371 \\
\hline & $\%$ diff & 0 & 0 & 0 & 0 \\
\hline \multirow{3}{*}{$\begin{array}{c}F_{P_{x}}^{g} \\
\text { (lbs) }\end{array}$} & HeliMan & -647.08002 & 5429.3944 & -982.25851 & 5177.1430 \\
\hline & & -647.079 & 5429.3949 & -982.25843 & 5177.1427 \\
\hline & & $\sim 0$ & $\sim 0$ & $\sim 0$ & $\sim 0$ \\
\hline \multirow{3}{*}{$\begin{array}{l}F_{P_{y}}^{g} \\
\text { (lbs) }\end{array}$} & HeliMan & 000.0000 & -2413.50317 & -6000.0000 & -6848.1857 \\
\hline & & 000.00000 & -2413.50324 & -6000.0000 & -6848.1857 \\
\hline & $\%$ diff & 0 & $\sim 0$ & 0 & $\overline{0}$ \\
\hline
\end{tabular}

In the above case descriptions, 'maximum' signifies that the point calculated was selected to be when the maximum force the RSD can produce has been reached. The corresponding probe deflection is corrected to keep the force at the specified maximum and not go beyond. This maximum force is limited by the hydraulic pressure used in the 
ASIST system and must only be applied when the RSD is generating the force. However, reaction forces in the probe can be generated that are larger than the specified maximum. Calculation point four shows that a force greater than the maximum probe force can exist in the RSD/probe model.

\subsubsection{Aerodynamic Model Comparison}

The verification results for the aerodynamic model are presented in Table 3.6. Equations 2.66 and 2.67 were used to calculate the drag forces, $F_{d x}^{h}$ and $F_{d y}^{h}$, acting on the helicopter caused by wind.

The test was performed with the helicopter in an aligned orientation with wind speeds of $10 \mathrm{ft} / \mathrm{s}$ in the forward direction and $15 \mathrm{ft} / \mathrm{s}$ in the starboard direction. The percent difference for each calculation has been found to be zero, therefore the aerodynamic model has been implemented correctly in HeliMan.

Table 3.6: Verification results for the aerodynamic model

\begin{tabular}{|c|c|c|}
\hline & Method & Calculation Point \\
\hline$V_{w x}^{h}$ & Input & $10.00000(\mathrm{ft} / \mathrm{s})$ \\
\hline$F_{d x}^{h}$ & HeliMan & 6.67836 \\
\cline { 2 - 3 }$(\mathrm{lbs})$ & Hand & 6.67836 \\
\cline { 2 - 3 } & \% diff & 0 \\
\hline$V_{w y}^{h}$ & Input & $-15.00000(\mathrm{ft} / \mathrm{s})$ \\
\hline$F_{d y}^{h}$ & HeliMan & -121.48988 \\
\cline { 2 - 3 }$(\mathrm{lbs})$ & Hand & -121.48988 \\
\cline { 2 - 3 } & \% diff & 0 \\
\hline
\end{tabular}

\subsubsection{Joystick Control Model Comparison}

The verification results for the joystick control model are presented in Table 3.7. The joystick function in Figure 2.15 was applied by using the maximum lateral velocities of $0.10833 \mathrm{ft} / \mathrm{s}$ to starboard and $0.13333 \mathrm{ft} / \mathrm{s}$ to port and maximum longitudinal velocity of 
$1.0 \mathrm{ft} / \mathrm{s}$. RSD travel limits were also applied and specified to restrict the RSD's motion to a two-foot square to demonstrate how they are implemented.

This test was performed for seven distinct situations starting with the helicopter and castor in an aligned orientation:

1. moving longitudinally against the RSD aft travel limit;

2. moving longitudinally towards the RSD forward travel limit;

3. moving longitudinally against the RSD forward travel limit;

4. moving laterally against the RSD port travel limit;

5. moving laterally away from RSD port travel limit;

6. moving laterally against the RSD starboard travel limit; and

7. traversing with float engaged.

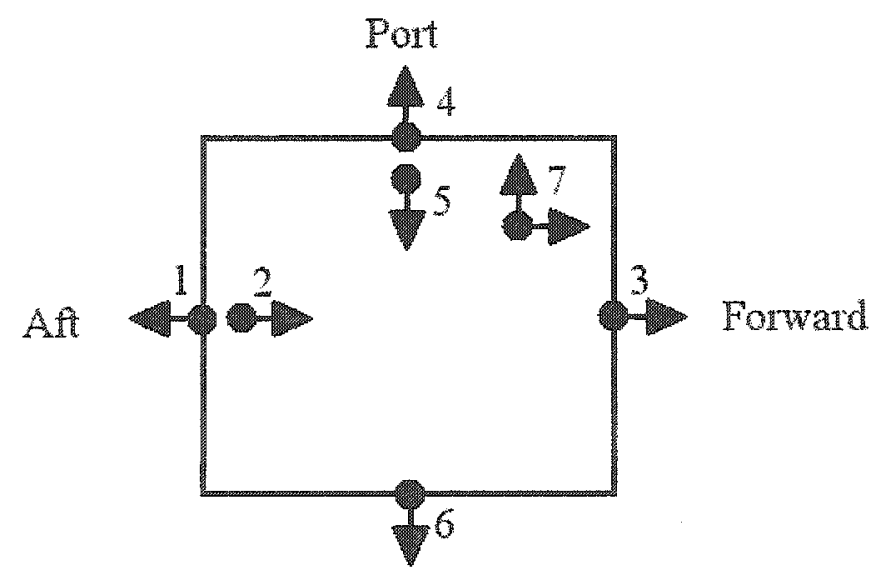

Starboard

Figure 3.2: RSD positions within the box corresponding to the different situations

The number of each situation corresponds to a calculation point in Table 3.7. Each situation is illustrated in Figure 3.2. In the situations when the RSD is at the limit, the model sets the velocity of the RSD in that direction to zero. The percent difference for 
each calculation has been found to be zero, therefore the joystick control model has been implemented correctly in HeliMan.

Calculation point seven demonstrates the application of the float function in the lateral direction. This was calculated using Equation 2.68 and 2.69. The lateral velocity is free to be as large as it needs to be to keep the lateral force at zero.

All of the important functions have been verified in this section. Less significant parts of the simulation have also been verified.

Table 3.7: Verification results for the joystick control model

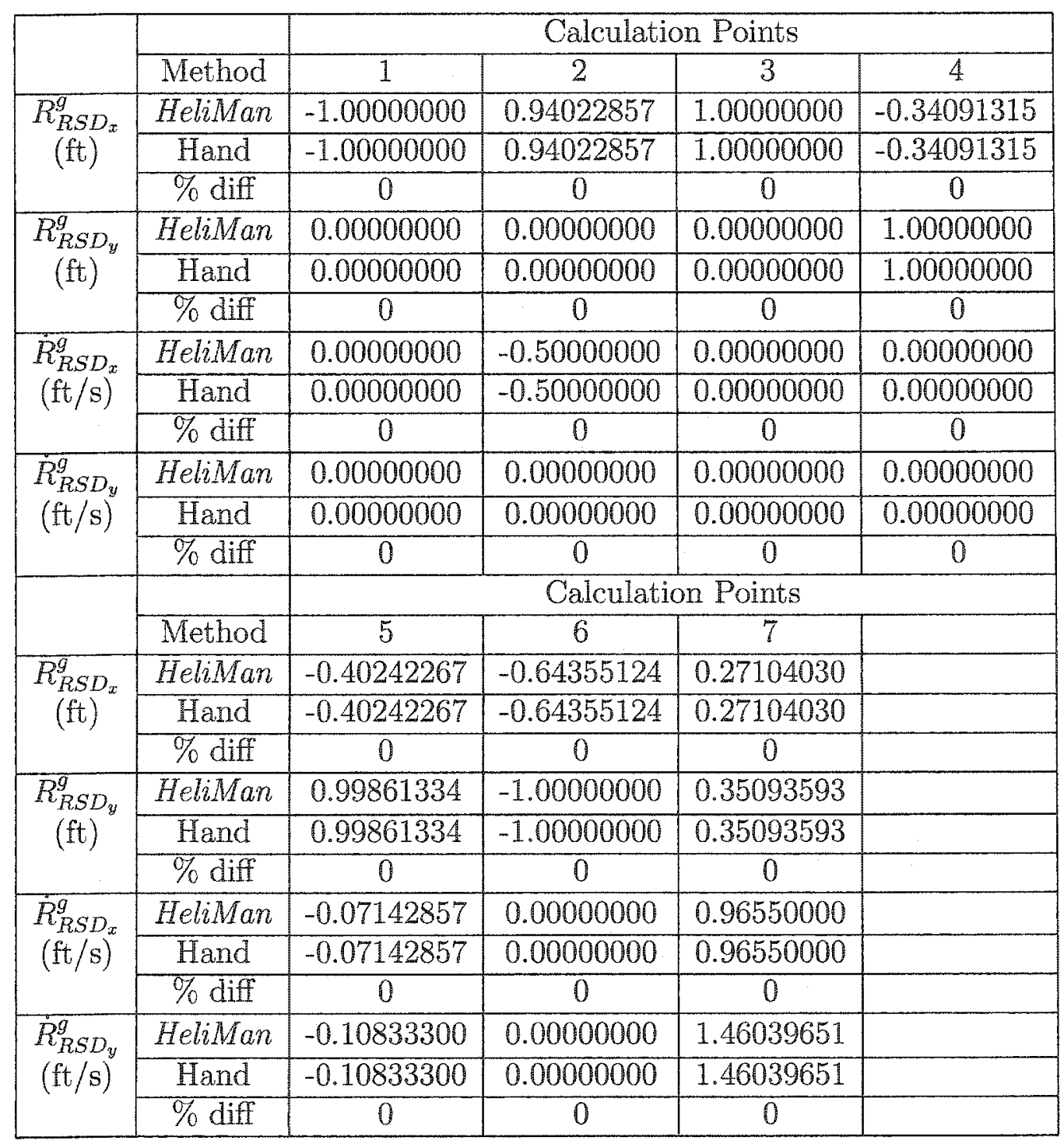




\subsection{Validation}

The validation of HeliMan used data generated from full-scale experimentation on ITI's ITF. The ITF does not perform tests in the presence of ship motion. Testing with ship motion was not needed because HeliMan is a planar simulation that does not include roll and pitch, which are dominant out-of-plane excitation sources caused by ship motion.

ITI's ITF was equipped with a DLTV, refer to Figure 3.3, configured for a Seahawk. Consequently, HeliMan was validated using the parameters for a Seahawk. The parameters used during the validation process will be discussed further in Section 3.2.3.

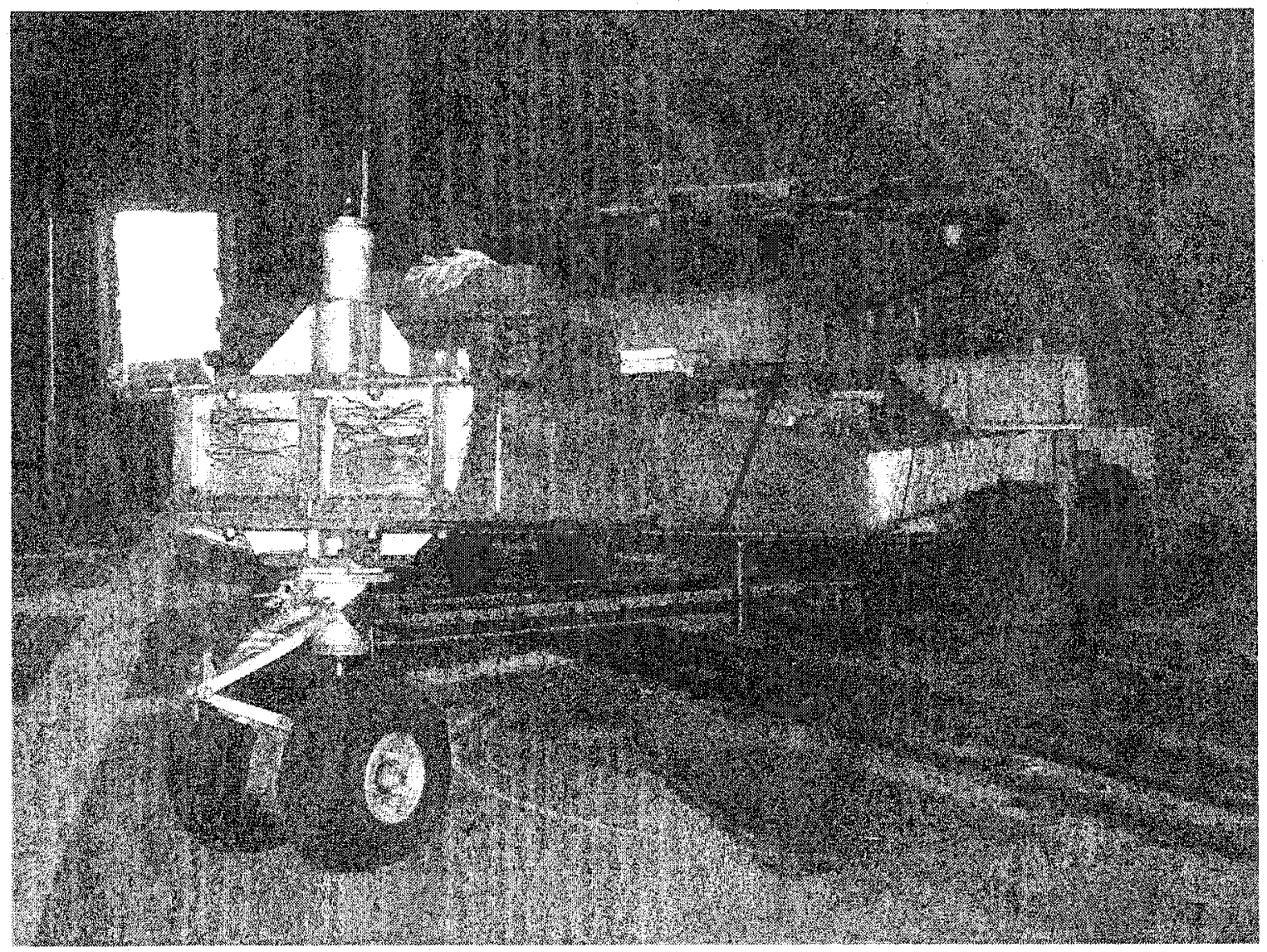

Figure 3.3: ITI's dead load test vehicle (DLTV)

A wide range of testing situations were planned and executed on the ITF. These test situations have been broken down into cases as follows: 
1. lateral stiffness;

2. traversing while yawed with a fixed castor assembly orientation;

3. yawed to 90 degrees;

4. yawed back to 0 degrees with free fioat;

5. rotating castor 180 degrees; and

6. traversing with lateral tire deflections and a tail probe.

The results from each case, along with more detailed descriptions are presented in Section 3.2.4. Due to time constraints on the availability of ITI's ITF, each testing situation was only completed once. Ideally each situation should have been repeated several times.

Four full straightening manoeuvres that would be seen during the practical application of the ASIST system where also performed. However, due to the nonlinear nature of the system and associated sensitivity to initial conditions, and the problems found in the preliminary validation of the cases outlined above, though simulated, they have not been included but are available for use in validating future refined versions of HeliMan. Little benefit would be gained by analyzing these cases with the simulation in its present state over those included in subsequent sections.

\subsubsection{Qualitative Validation}

The joystick and graphical interface have been used continuously during the development of Heliman to verify the simulation qualitatively. Any significant discrepancies seen during this process where addressed in the models. An experienced ASIST operator was also able to test Heliman periodically and provide qualitative recommendations. This qualitative Validation process was found to be invaluable in the development of HeliMan. 


\subsubsection{Partial Tire Validation}

Tire data was made available by the manufacturer for the tires used on the DLTV. The parameters available are the vertical force as a function of the vertical deflection and the lateral tire stiffiness as a function of the vertical force for the main and tail tires.

In Figure 3.4 the empirical relationship developed by Smiley and Horne [34] for the vertical tire force and deflection, Equation 2.37, is compared to the manufacturer's curve for the main tire. Figure 3.5 is the same plot, but for the tail tire. Both plots correspond very well with the manufacturer's curves.

In Figure 3.6 the empirical relationship developed by Smiley and Horne [34] for the vertical tire force and the lateral tire stiffness, Equation 2.39, was compared to the manufacturer's curve for the main tire. Figure 3.7 is the same plot, but for the tail tire. The main tire corresponds very well with the manufacturer's curve. However, the tail tire only fits moderately well. It is interesting to point out how the tire stiffness counterintuitively decreases as the vertical force increases.

Both empirical relationships have been found to be reasonably valid for the tire model implemented in HeliMan. The empirical equations presented by Smiley and Horne were developed for a very wide range of tire sizes and types. Small to moderate discrepancies can be expected from an empirical relationship derived for many different tire types. Manufacturer data was only available for these two parameters, so the direct tire validation was limited to that presented in this section. 


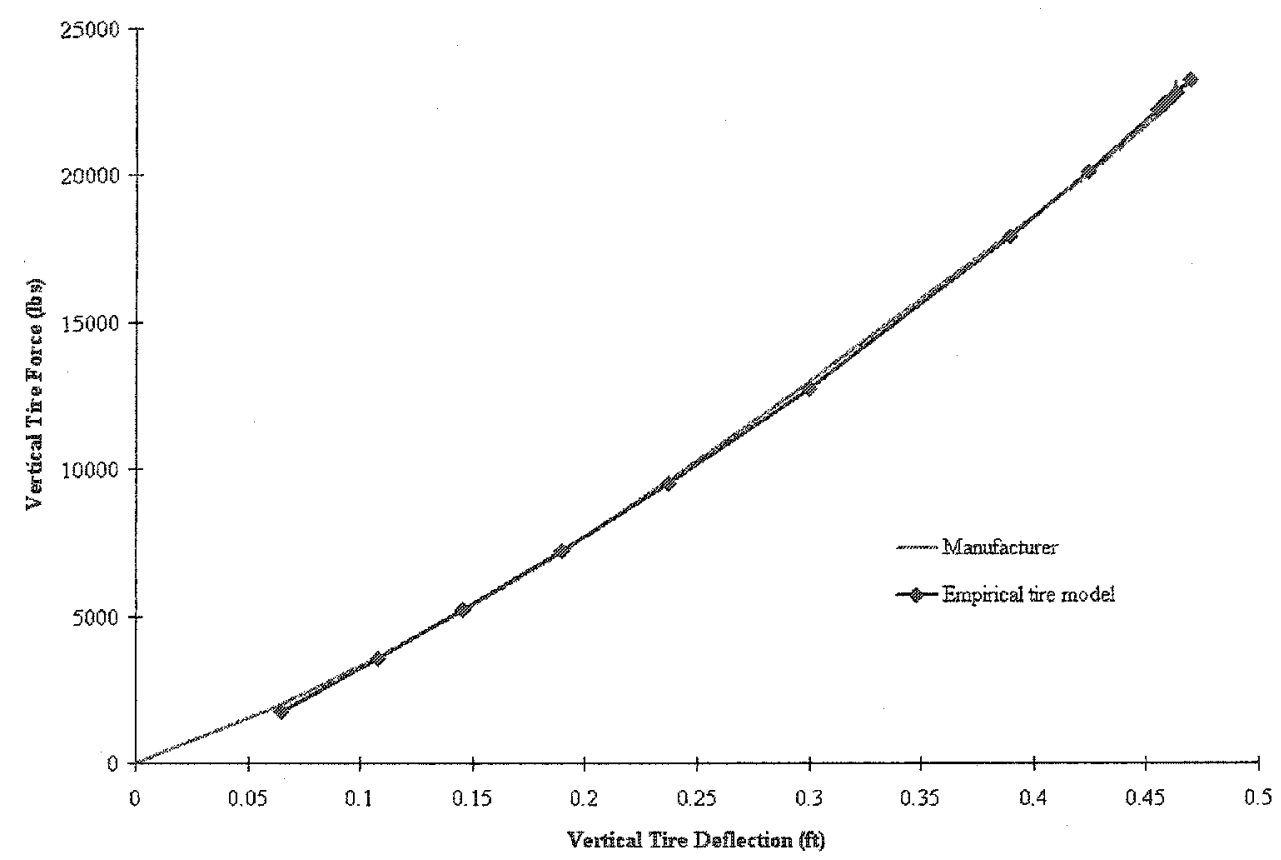

Figure 3.4: Vertical tire deflection versus vertical tire force for the main tire

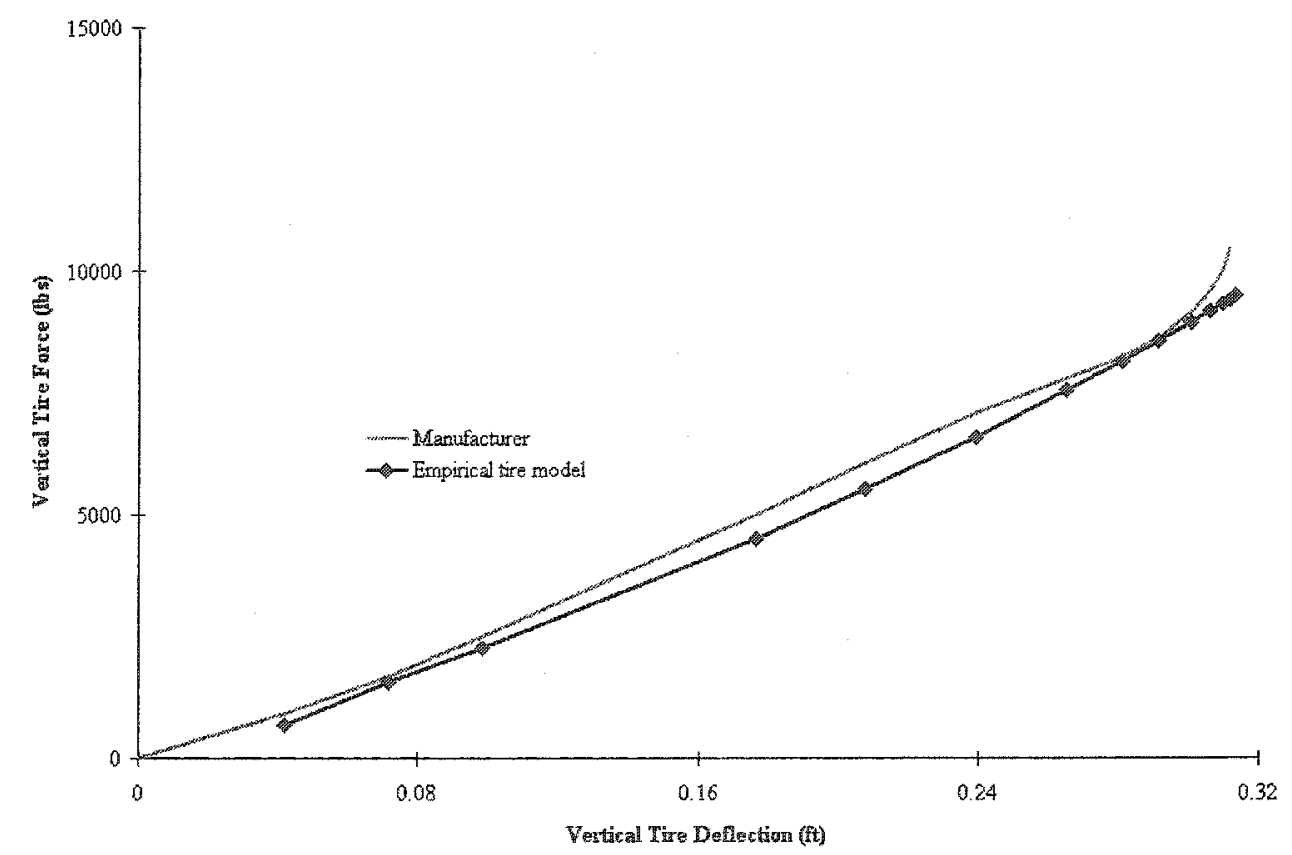

Figure 3.5: Vertical tire deflection versus vertical tire force for the tail tire 


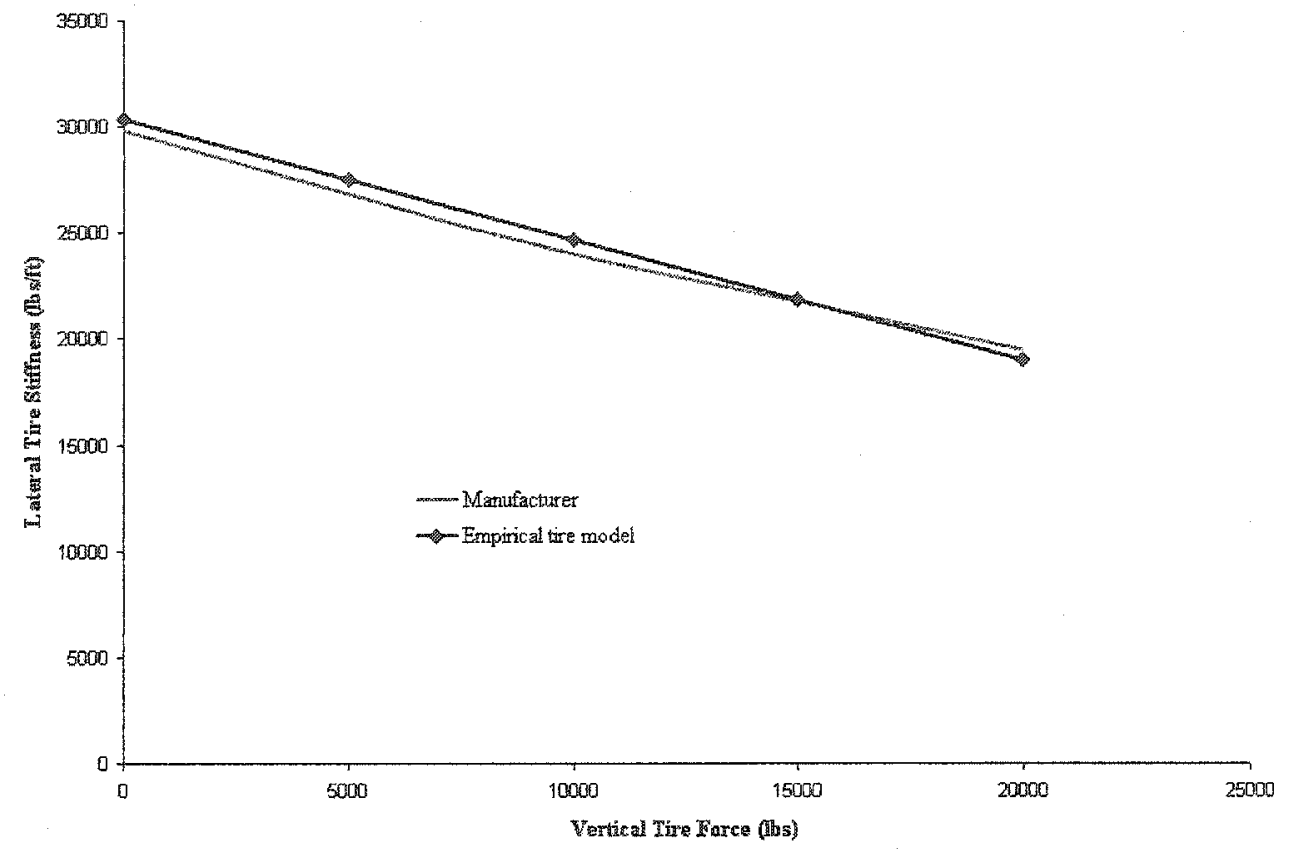

Figure 3.6: Lateral tire stiffness versus vertical tire force for the main tire

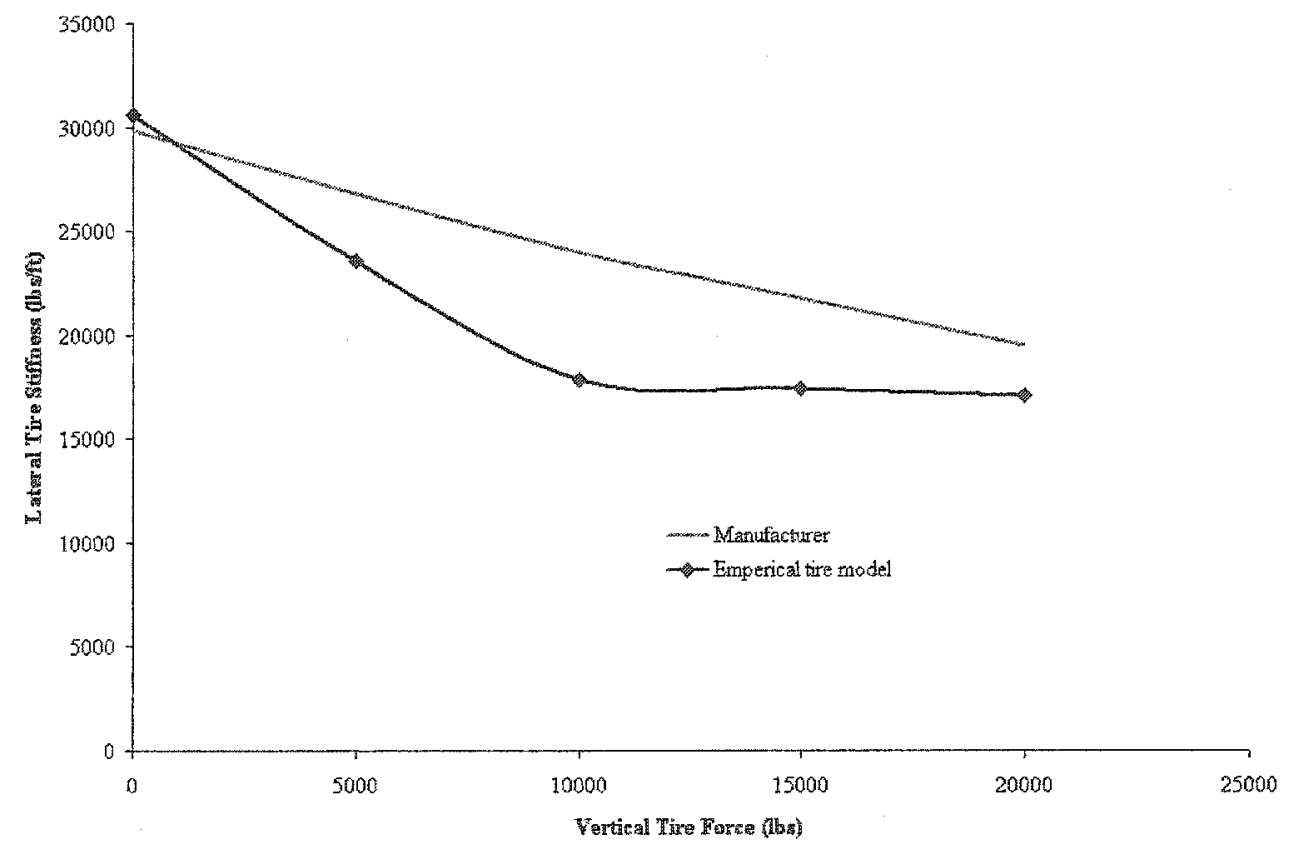

Figure 3.7: Lateral tire stiffness versus vertical tire force for the tail tire 


\subsubsection{Validation Parameters}

Before any testing began, the parameters of the DLTV were recorded and compared with the values for the real Seahawk. The wheel base and track width were both measured by hand and found to be consistent with the actual values. The geometry of the Seahawk castor assembly was not known but was measured. The weight distribution was available and was consistent with the real Seahawk. An estimate of the castor assembly weight was also available. The DLTV also has the same tires installed as would be on the real Seahawk. The tire pressures were recorded and used in the simulation.

During testing the following data was gathered from the ITF for each case:

- longitudinal RSD claw velocity;

- lateral RSD claw position;

- longitudinal and lateral force components acting on the probe measured in the helicopter frame of reference;

- helicopter position measured by hand at discrete times (used as a check for the first item);

- helicopter orientation measured by hand at discrete times;

- lateral deflections of the main tires; and

- castor wheel orientation up to \pm 180 degrees.

This data is presented in plotted form in Section 3.2.4 for each case. The initial values for each case are used to set up the starting conditions of the simulation for each case.

There are a number of values that were not gathered, but are needed to complete a more thorough validation of HeliMan. While these requirements were identified in the test plan, data collection was limited by available ITF instrumentation. For example, if the DLTV orientation could be measured in a continuous manner there would be no need to stop the test and measure these values by hand. It was not known when the float function is being used by the operator, so that aspect of the simulation could not be validated. 
No lateral deflections of the tail tires were recorded. Sometimes during full straightening manoeuvres, the castor wheel needs to be realigned almost 360 degrees in one direction. In the current configuration if this was done, the castor sensor would be damaged. So for the full straightening manoeuvres the sensor was removed.

\section{Simulation Inputs}

The longitudinal RSD claw velocity and lateral RSD claw position were used to generate an input file for each validation case. The longitudinal RSD claw velocity was integrated and verified against the measured distances. A continuous differentiation of the lateral RSD claw position was used to determine the lateral velocity of the RSD claw. This input file was then used to bypass the joystick model in HeliMan by applying the RSD claw velocities directly in the simulation.

Theoretically, by doing this, the problem of not knowing when the float function was applied was solved. Any time the float function was being used, the corresponding RSD claw velocity would be applied. It still means that the operation of the float function in the simulation could not be validated. For that, an indication of when it was being applied during the validation experiments was needed.

\section{Integration Time Step Selection}

A simple run was performed with HeliMan to determine an appropriate time step for the fourth-order Runge-Kutta integrator. The helicopter was manipulated from the aligned position to an orientation of approximately 22 degrees. This manoeuvre was repeated using different time steps and the final orientation of the helicopter using each time step was compared.

Figure 3.8 shows the results of this comparison. When the time step is chosen to be greater then approximately $0.6 \mathrm{~ms}$, large discrepancies in the final helicopter orientation 
begin to appear. The solution results for time steps below $0.6 \mathrm{~ms}$ converge at approximately 22 degrees. The point at which the solution converges may vary in different manoeuvring situations. Therefore a time step of $0.1 \mathrm{~ms}$ was used during the validation process and it is recommended that no time step greater then $0.4 \mathrm{~ms}$ be used.

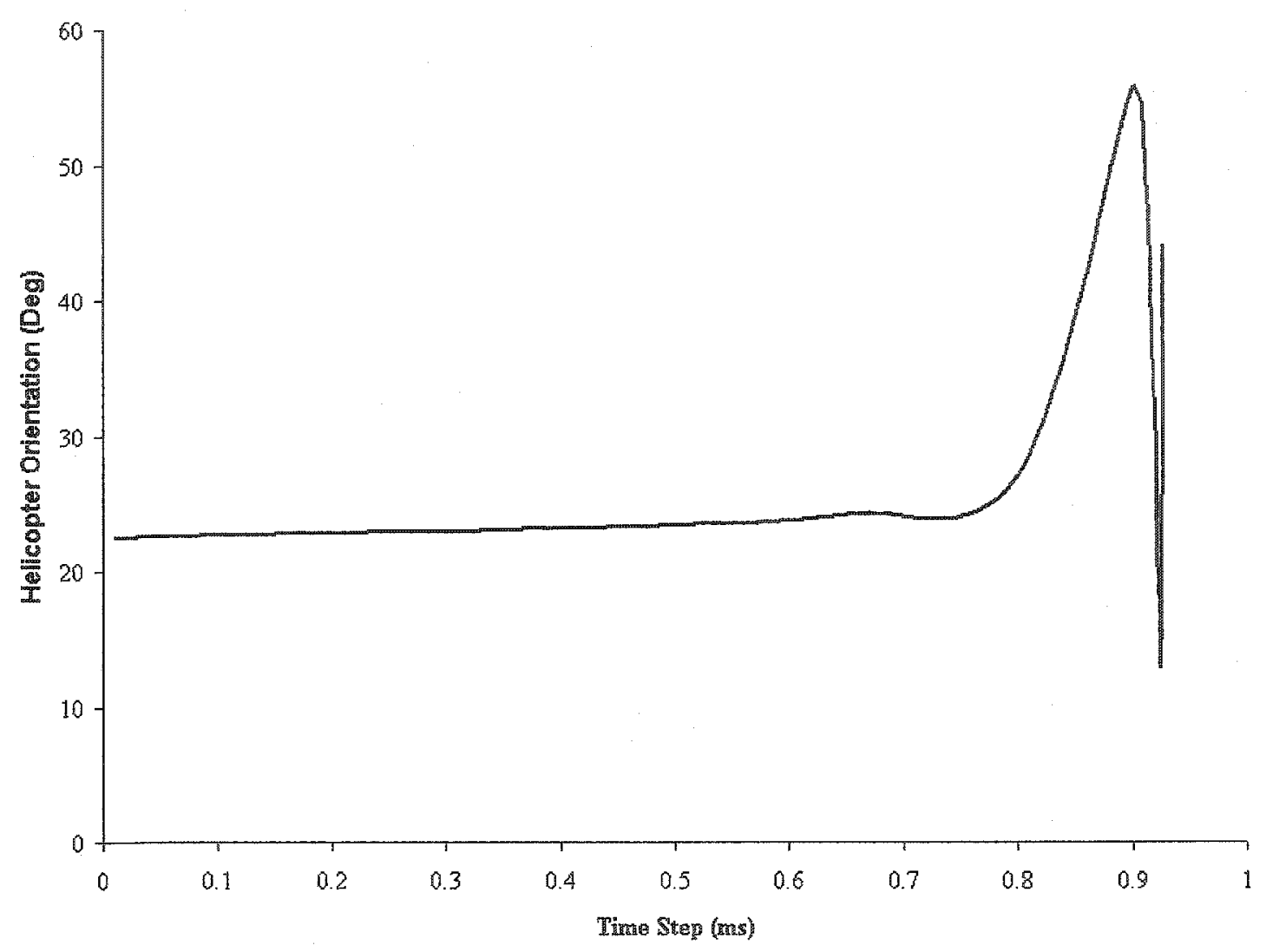

Figure 3.8: Comparison of manoeuvre results using different time steps

\section{Mass Moment of Inertia}

The mass of the DLTV was the same as a Seahawk, but the mass moment of inertia was not known. The actual mass moment of inertia for the Seahawk was known. The DLTV was constructed to mimic the footprint and weight distribution on each wheel of the Seahawk. However, the weight was not distributed to replicate the proper mass moment of inertia. 
For this reason the mass moment of inertia of the DLTV was most likely different than that of the Seahawk.

A body such as the helicopter or DLTV can be represented by a ring with the same mass evenly distributed around its circumference. The radius of this ring is known as the radius of gyration and is directly proportional to its mass moment of inertia, $k_{h}=\sqrt{\frac{I_{h}}{m_{h}}}$. The radius of gyration for the Seahawk was found to be approximately nine feet. Theoretically, if the weight was distributed on the DLTV in a ring with a 9 foot radius its mass moment of inertia would be the same as the actual Seahawk. However, the current DLTV would be able to fit inside this ring making its mass moment of inertia smaller than the actual Seahawk.

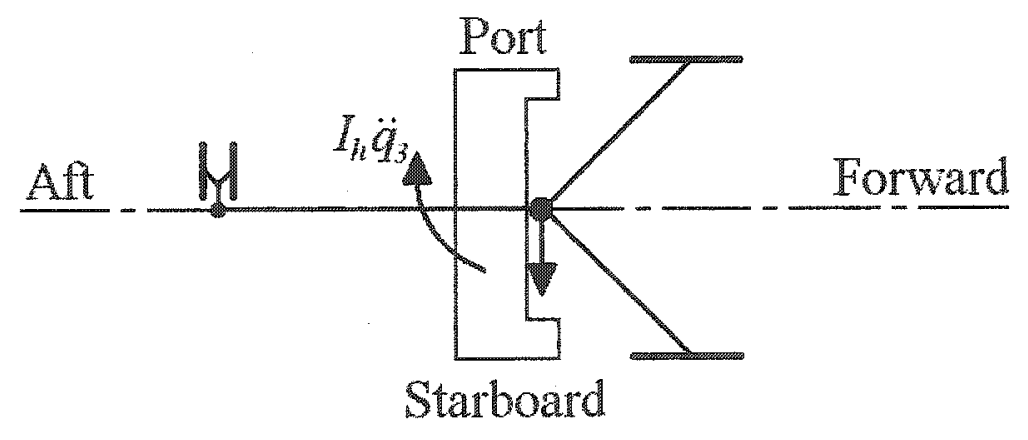

Figure 3.9: Initial position of helicopter and the direction RSD is moved in HeliMan to test the effect of changing the mass moment of inertia

A simple test was conducted using HeliMan to find out what the effect of having a lower mass moment of inertia would be. The helicopter was yawed to the port side as illustrated in Figure 3.9. The D'Alembert inertia moment shown in Figure 3.9 is calculated for the actual mass moment of inertia, seventy-five percent of the actual value and ten percent of the actual value. The helicopter inertia moment variations with time are compared in Figure 3.10. While the initial amplitudes with the full Seahawk mass moment of inertia are similar to seventy-five percent of the full value, the smaller value results in faster dissipation of the inertia moment. Using ten percent of the actual mass moment of inertia 
significantly effects the amount of inertia moment the helicopter experiences.

The difference in the mass moments of inertia will have an effect on the validation cases where the DLTV is being yawed. This effect will be amplified by all the stopping and starting being done to measure the DLTV's position and orientation. Each time the simulation stops or starts this D'Alembert inertia moment needs to be dissipated or overcome. Therefore the simulation results using the actual mass moment of inertia will be much more choppy than the experimental results. This will especially be noticeable in the cases where the DLTV was stopped a lot to perform hand measurements.

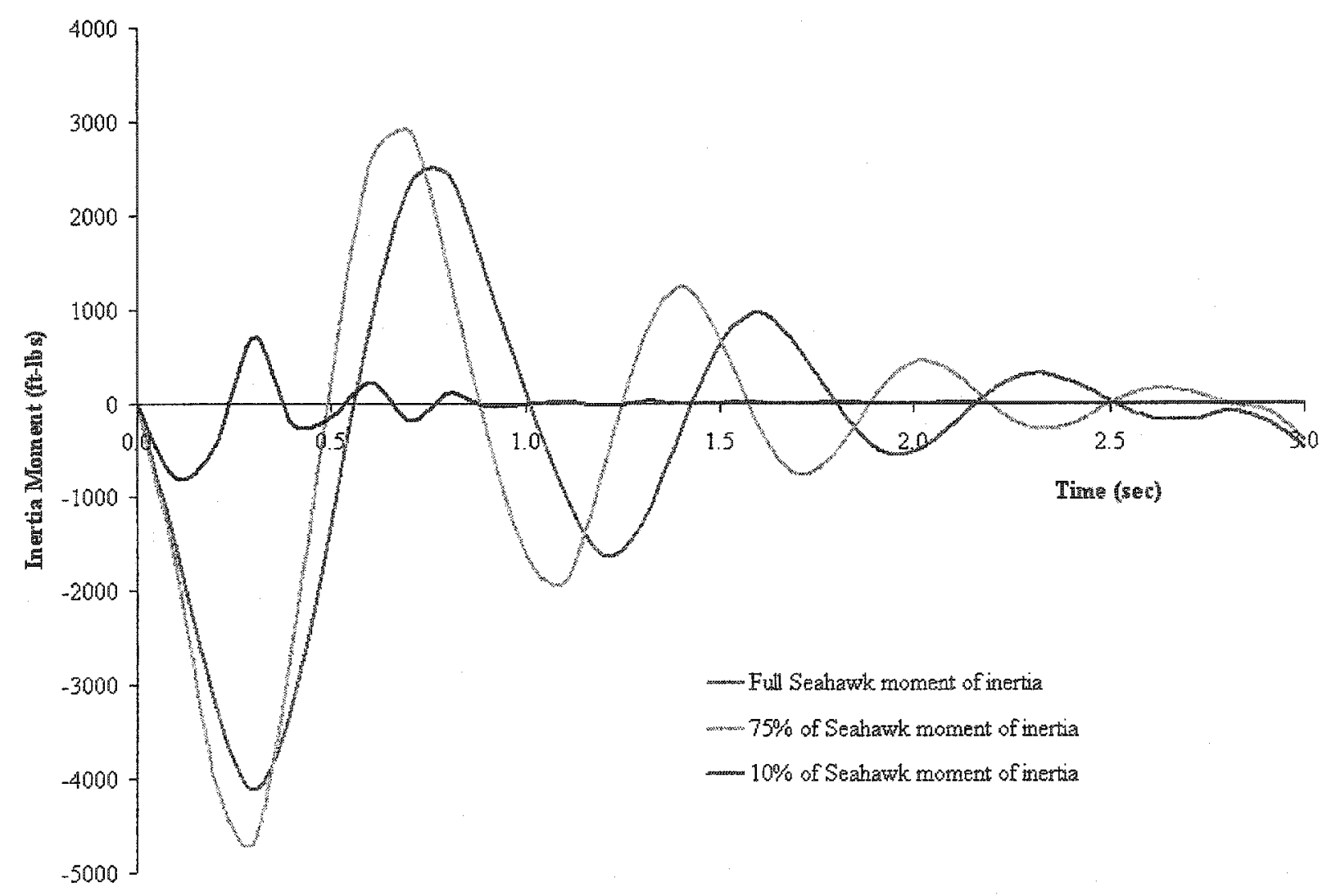

Figure 3.10: The effect of lowering the mass moment of inertia in HeliMan

Because the actual value of the DLTV mass moment of inertia is unknown, the actual Seahawk value will be used in the simulation. In validation Case 3, results are generated 
for the actual mass moment of inertia and for ten percent of the actual value.

The mass moment of inertia of the castor assembly was also not known for either the real Seahawk or the DLTV. It has been observed that the mass moment of inertia of the castor assembly has an insignificant effect on the simulated helicopter response when compared to that of the main helicopter body.

\section{Rolling Resistance Test}

A simple test was performed to determine the total rolling resistance force acting on the DLTV. This test could not be treated as a full validation case because the only values recorded were the $\mathrm{x}$ and $\mathrm{y}$ component forces acting on the main probe in the DLTV frame of reference over time. The DLTV was traversed along the full length of the ITF track back and forth twice with the castorable tail assembly fixed and the tail probe engaged. Figure 3.11 shows the initial position of the DLTV.

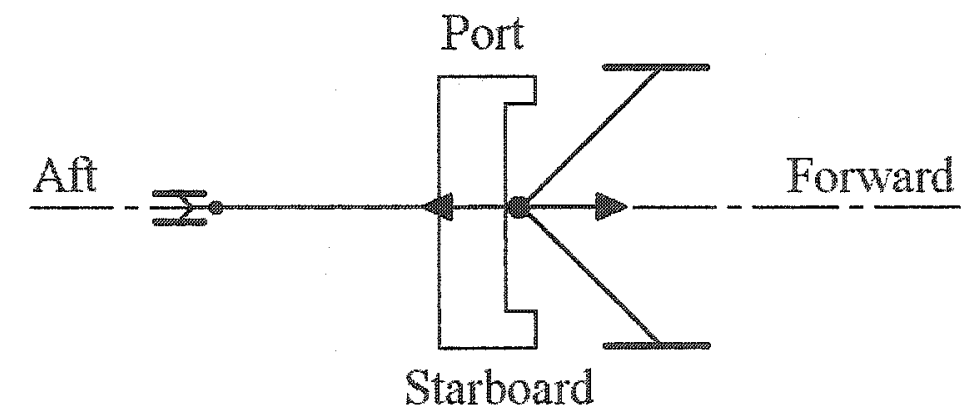

Figure 3.11: Initial position of DLTV and direction RSD is moved for the rolling resistance test

Figure 3.12 is a graph of the results obtained from this test. In the Figure, the $\mathrm{x}$ main probe force is black while the y main probe force is grey. The traversing stages are indicated by the dashed lines. The ITF track has a curved portion near its forward end. The jump in forces at times 40 to 60 seconds and 115 to 135 seconds are caused by the curved portion of the ASIST track. 
From Figure 3.12, the instability of the helicopter being traversed in the forward direction compared to the aft direction can be seen in the build-up of a $y$ force on the main probe. The helicopter has a tendency to yaw around to its stable configuration of the tail wheel leading. Theoretically this will not happen if the helicopter is perfectly aligned, but this condition is impractical to achieve in reality. The tail probe prevents this unstable yawing effect as well as allowing the helicopter to follow the curved track.

To determine the rolling resistance coefficient, only the force acting on the main probe in the $\mathrm{x}$ direction was needed. The data collected for sections of curved track were omitted and the force acting on the main probe in the $\mathrm{x}$ direction was plotted on its own in Figure 3.13. A consistent value for the total rolling resistance force acting on the DLTV was evident from Figure 3.13. The 10 to 45 second section has been enlarged in Figure 3.14.

An approximate value of 300 lbs total rolling resistance acting on the DLTV has been determined from Figure 3.14. Equation 2.53 was then used with the weight to determine an approximate value of 0.014 for $\mu_{r r}$. This coefficient was used for each tire type and for the analysis of validation case results.

It is possible that the main and castor tires could have different coefficients of rolling resistance. As long as the total amount of rolling resistance force induced by all four tires is the same, this possibility will not have an effect on a helicopter being traversed along a straight line. However, it would be important when the tires are rolling in different directions, such as when the helicopter is being manipulated laterally. At this point HeliMan uses the same coefficient of rolling resistance for both main and tail tires. 


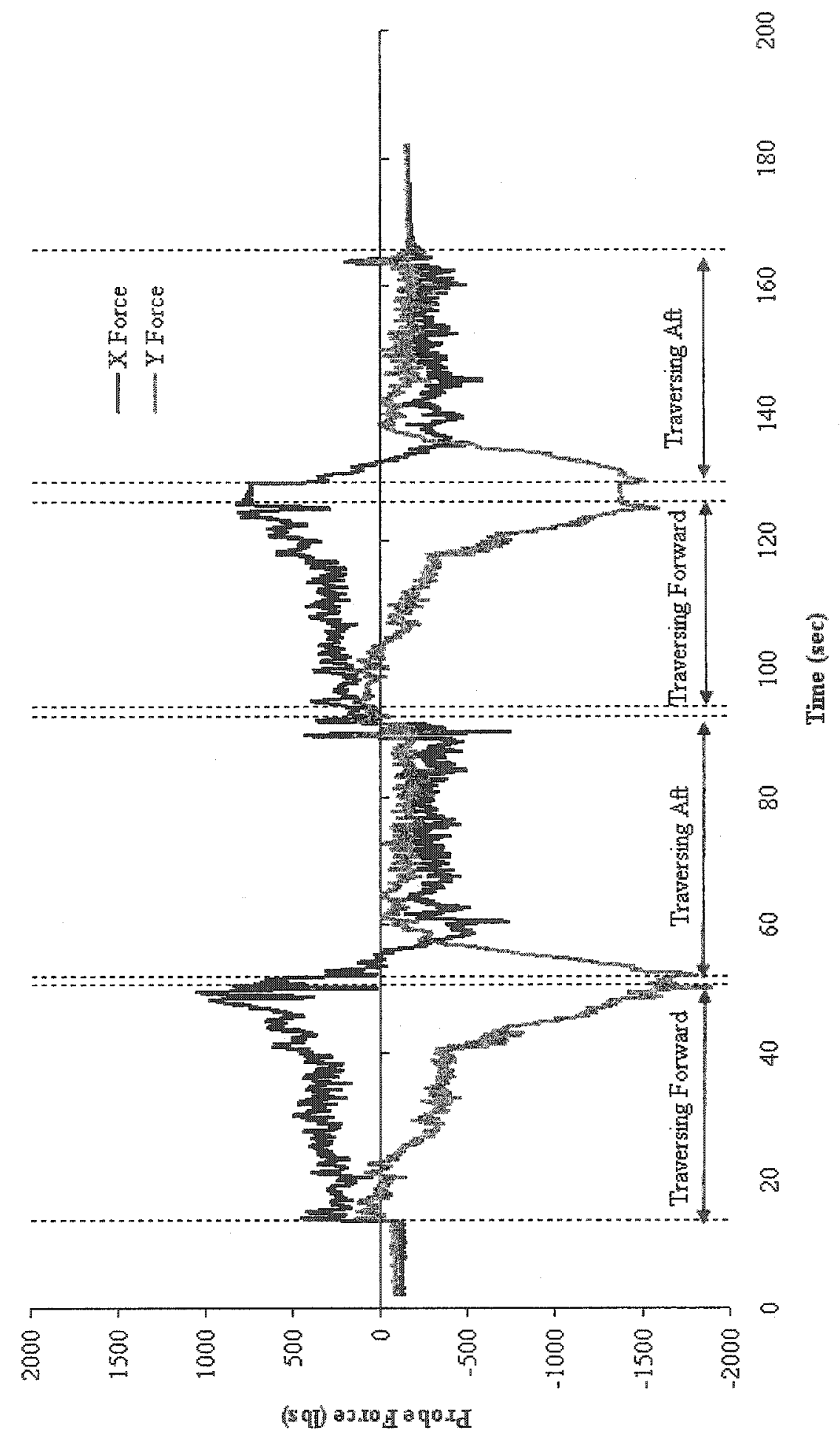

Figure 3.12: Component forces acting on the main probe for the rolling resistance test 


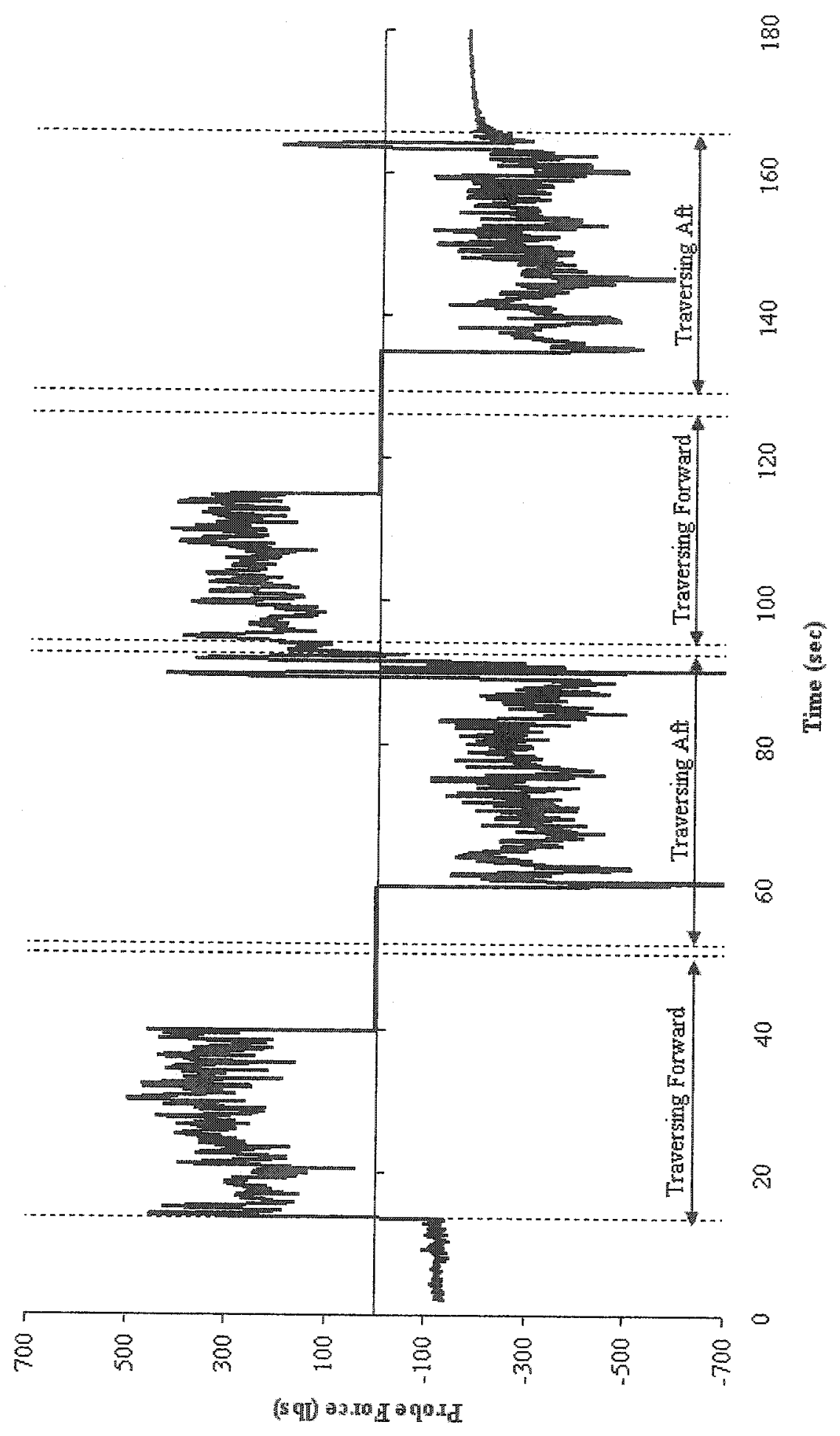

Figure 3.13: X component forces acting on the main probe during the straight track portions 


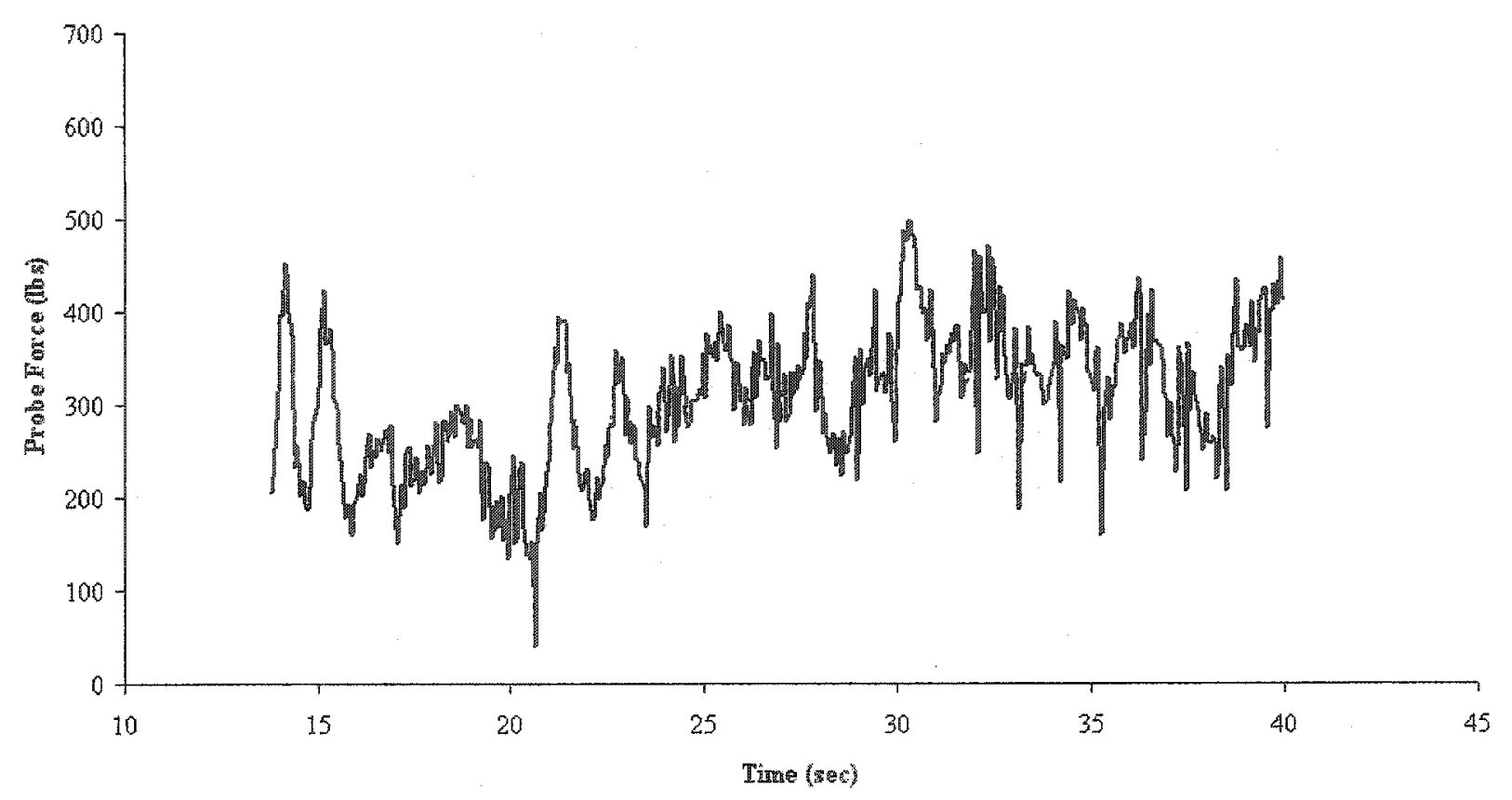

Figure 3.14: $\mathrm{X}$ component forces acting on the main probe during the first traversing stage

\section{Unknown Parameters}

The probe stiffness and damping values, $K_{P_{x}}, K_{P_{y}}, C_{P_{x}}$ and $C_{P_{y}}$, needed to determine the effective stiffness and damping forces have been found by tuning the simulation against the experimental data. The small deflection values for the probe, $K_{P s_{x}}, K_{P_{s_{y}}}, D_{P_{s_{x}}}$, and $D_{P s_{y}}$ were known. These values represent the stiffness and damping of the entire RSD structure, DLTV frame, and the securing system. The specific values used depend upon the securing system and in this case are proprietary to ITI.

The effective castor joint coefficient for Coulomb friction, $\mu_{B} r_{\mu}$, was found to be 0.12 $\mathrm{ft}$ and effective viscous damping coefficient, $C_{B} r_{\mu}{ }^{2}$, was found to be $2000 \mathrm{lb} \mathrm{ft} \mathrm{s}$. The corresponding radius is combined with the coefficient because both are unknown. These coefficients were determined for the Seahawk by tuning the simulation with the experimental data. The kingpin inclination angle is known for a Seahawk. 
The same values have been used for all of the validation cases. Consequently, a compromise had to be met on these values so that reasonable results could be obtained for all six validation cases. It is possible that these values are compensating for limitations within the simulation. If these limitations are corrected these values will need to be reevaluated.

\subsubsection{Validation Results}

The results from the six cases tested are presented and discussed in this section.

\section{Case 1: Lateral Stiffness}

For this test case the DLTV was located and oriented as shown in Figure 3.15 with an orientation angle of $13^{\circ}$ to starboard. The castor assembly was also locked in the aligned position.

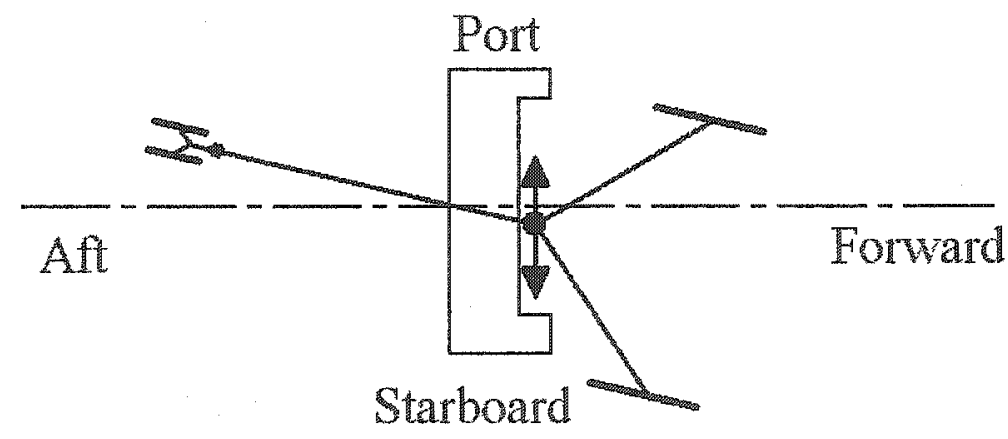

Figure 3.15: Initial position of DLTV and direction RSD is moved for Case 1

The purpose of this case was to test the lateral stiffness of the entire system. Once the DLTV was set up in its initial position, the operator moved the RSD laterally back and forth to build probe forces in the lateral direction. At the end of the test case, the operator used the float function to dissipate the accumulated force. This was done continuously as it was assumed that the longitudinal position of the RSD and the DLTV orientation would stay constant. 
Figure 3.16 shows the lateral position of the RSD claw. The inputs being input into the simulation match perfectly with the experimental data. The RSD claw started out 4 inches to the starboard side. Next, the operator manipulated the RSD claw back and forth in the lateral direction. At around 60 seconds the operator used the float function to dissipate the force that was built up. The RSD claw was then moved to the starboard side and the float function was used again.

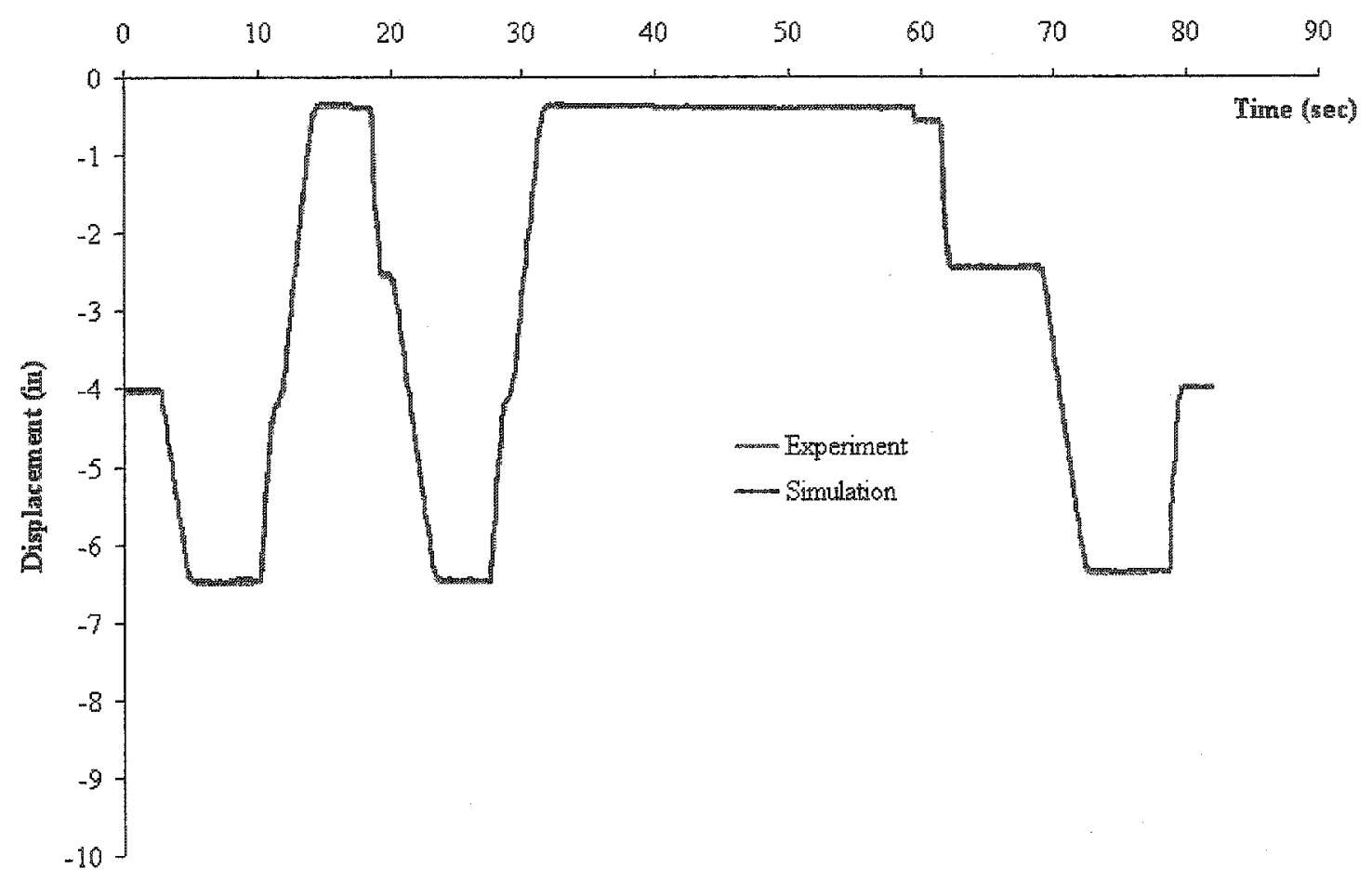

Figure 3.16: Comparison of the RSD claw y-position for Case 1

Figure 3.17 shows the probe force in the DLTV y-direction with the positive y axis being perpendicular to the DLTV and pointed out the port side. The simulation matches closely with the experimental results. Some inconsistences can be observed when the float function was used.

The oscillations in the experimental data are caused by the suspension on the DLTV. Heliman does not currently model the suspension dynamics of the helicopter, so any 
oscillations caused by the suspension will not occur in the simulation.

Figure 3.18 shows the probe force in the DLTV $\mathrm{x}$-direction. A significant difference exists between the experimental data and that generated by the simulation. It is believed that the problem was caused by a limitation in the tire model. In the tire model it was assumed that the tire just rolls in the longitudinal direction no matter how large the lateral force is. Consequently, the only longitudinal force the tires experience in HeliMan is the rolling resistance. A new tire model component was derived to try to improve the tire model performance and is presented after the lateral tire deflections are discussed.

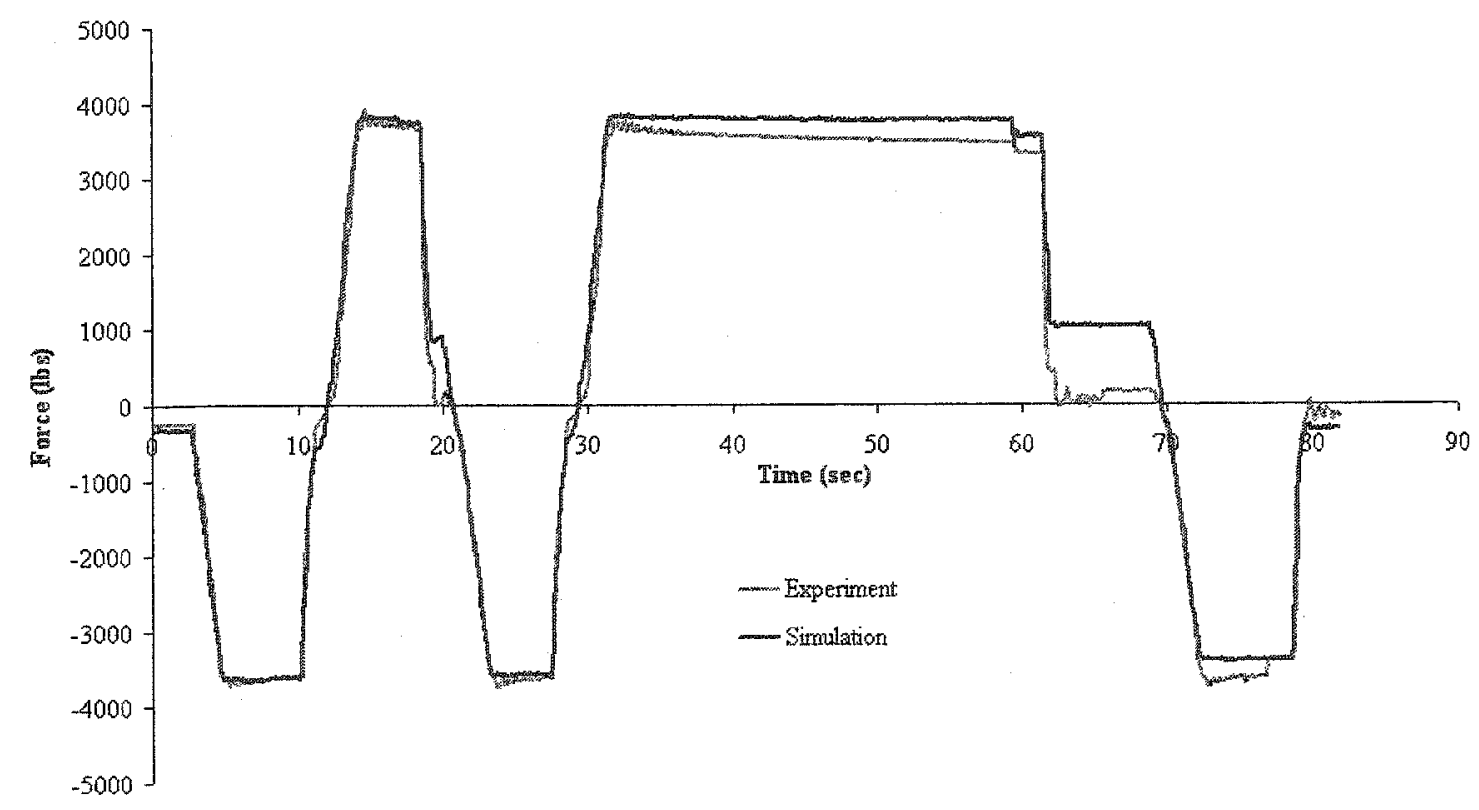

Figure 3.17: Comparison of the force acting on the main probe in the DLTV's $y$-direction for Case 1

Figure 3.19 shows plotted results for the lateral tire deflections for the main gear. Over the first few seconds the lateral deflections generated by the simulation tend to balance out. This tendency is shown more clearly in Figure 3.20. If the two main tires have opposite lateral deflections of significantly different magnitudes the tendency of the system is to adjust the position of the helicopter body and balance the magnitudes out.

This tendency to balance the magnitude of the lateral tire deflection causes the offset 


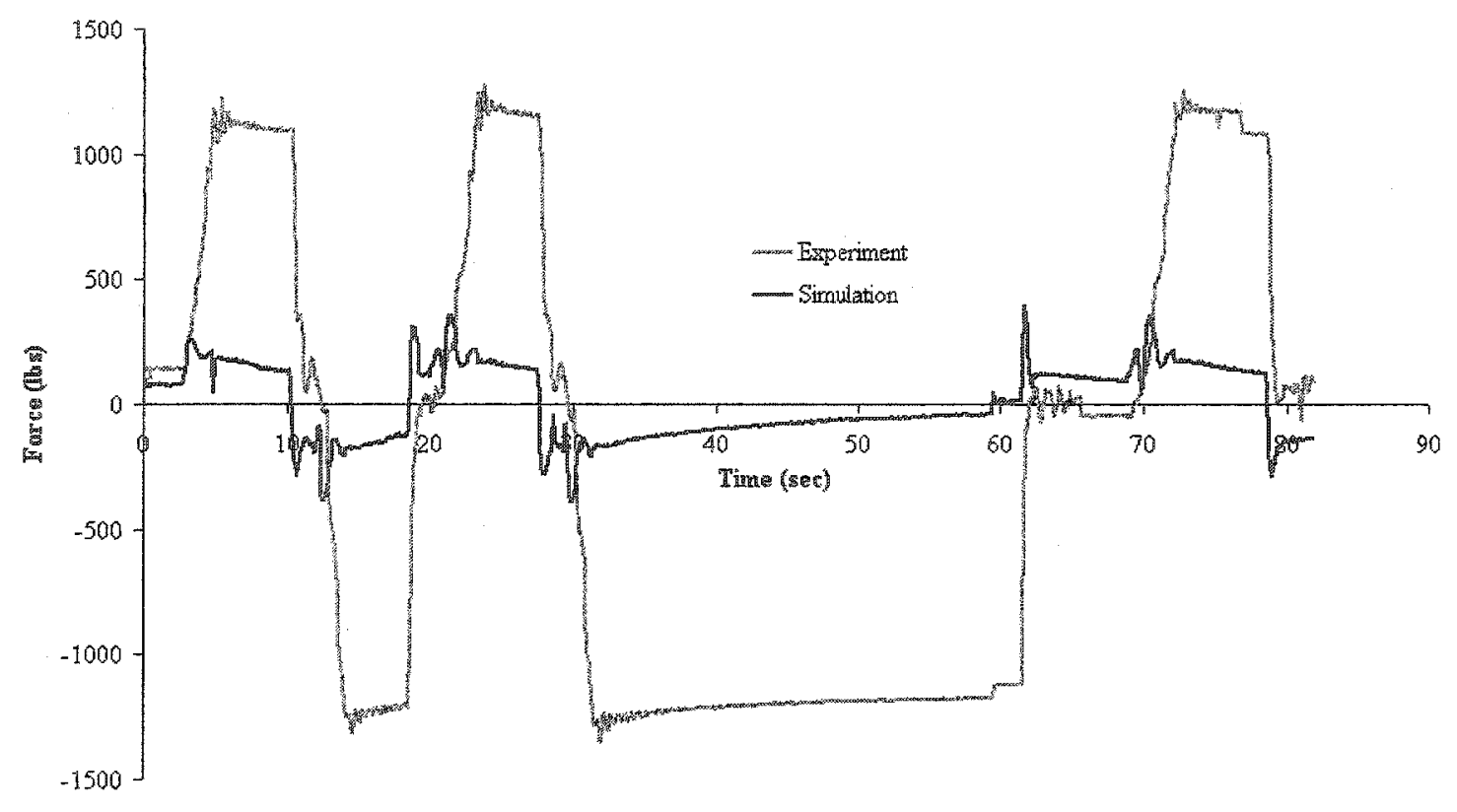

Figure 3.18: Comparison of the force acting on the main probe in the DLTV's $\mathrm{x}$-direction for Case 1

in the lateral deffections observed in Figure 3.19. The experimental data did not show this tendency. This could be because deflections on the castor tires, which were not recorded, are counteracting the tendency. The simulation was run under the assumption that the lateral deflection of the castor tires were zero, which is almost certainly false. It is also possible that the simulation is overestimating this tendency. 


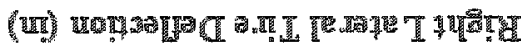

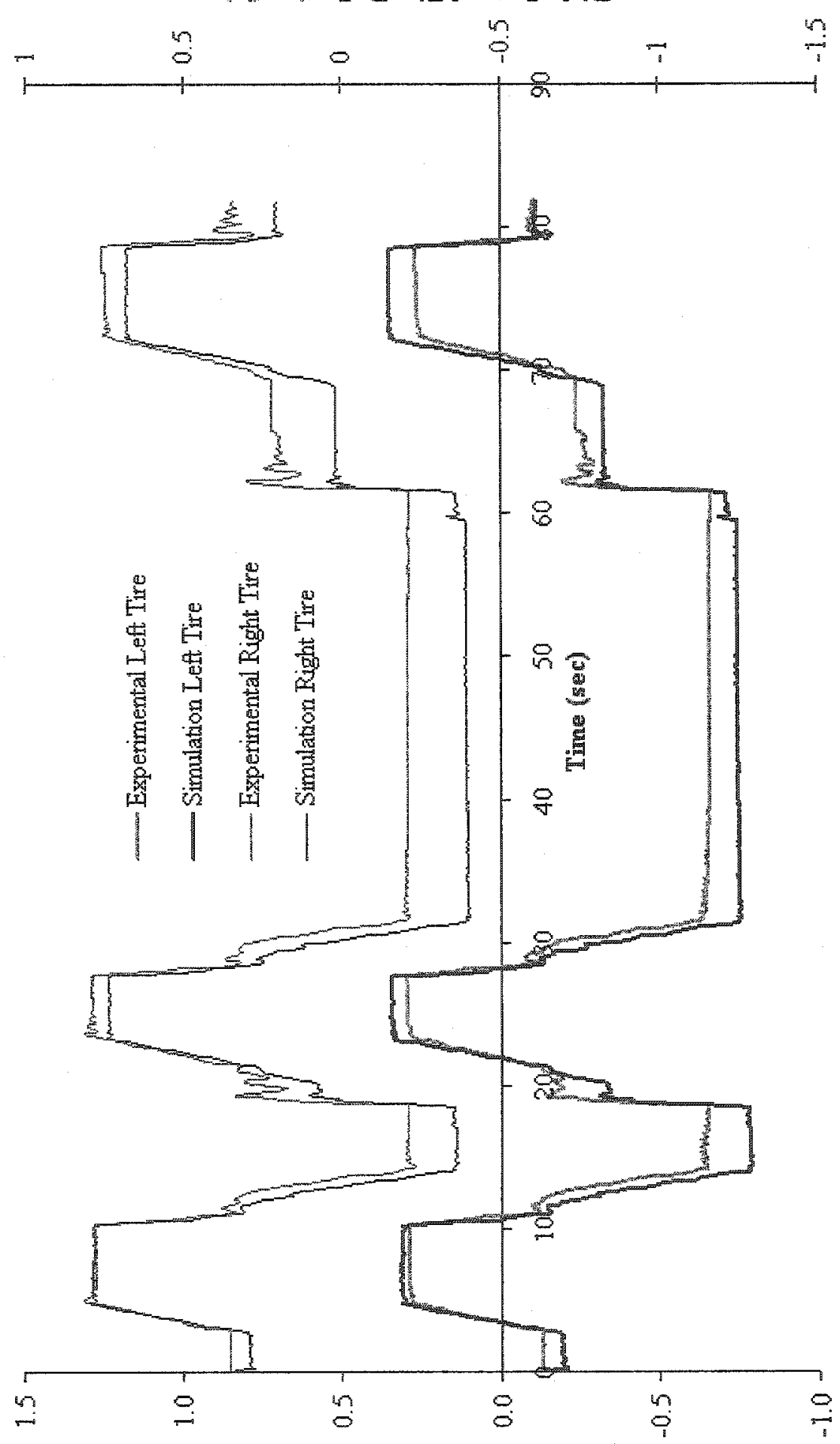

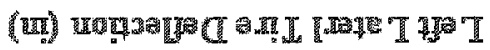

Figure 3.19: Comparison of the lateral tire deflections for Case 1 


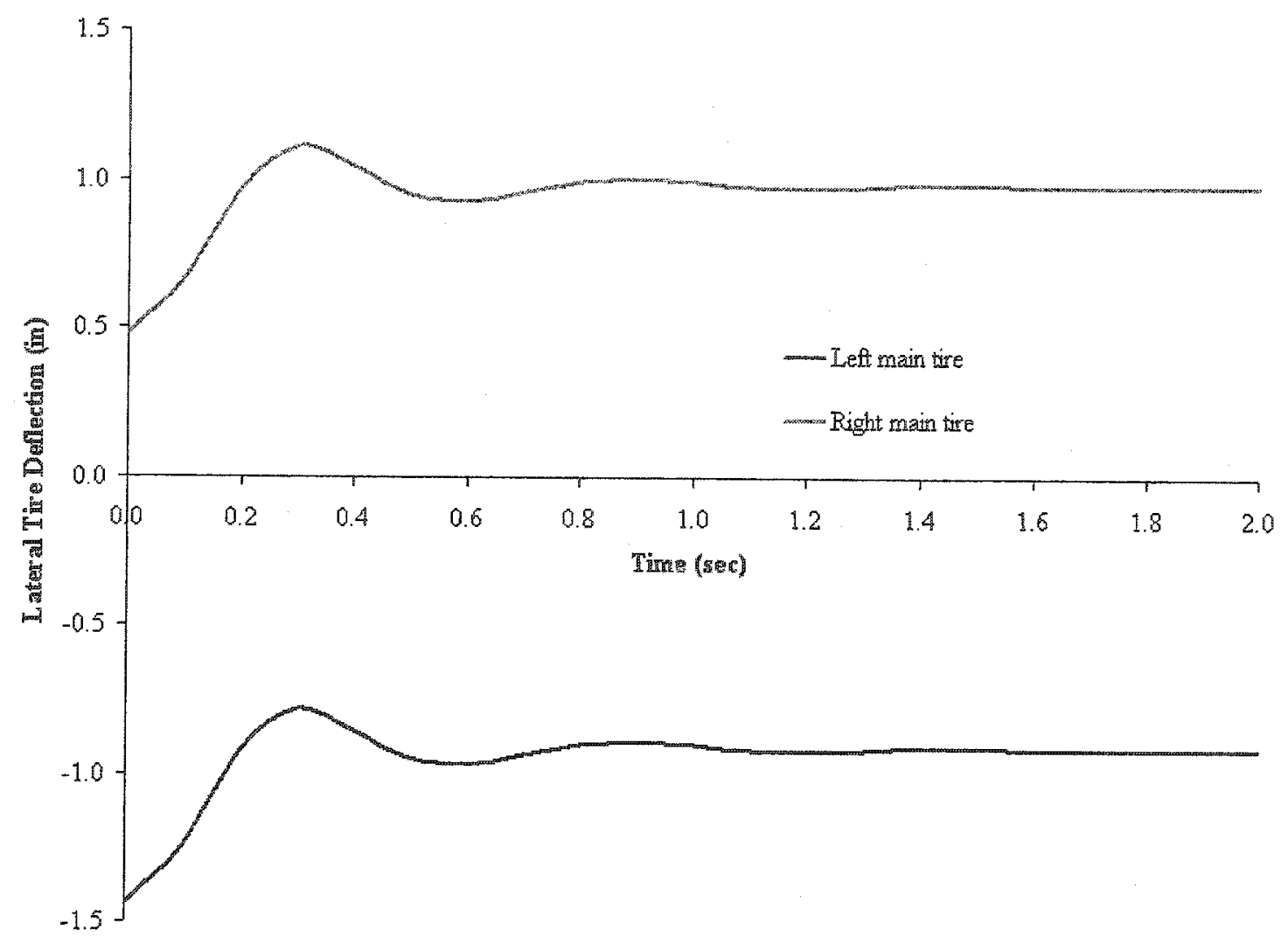

Figure 3.20: Demonstration of the simulations tendency to balance out the lateral tire deflections

A component for the tire model was derived to address the problem with the main probe force in the $\mathrm{x}$-direction observed in Figure 3.18. It was speculated that the lateral force acting on the tire causes a couple on the wheel axle. This couple was then used to add a frictional resistance force between the axle and the wheel. This force balance is shown in Figure 3.21. The axle frictional force, $F_{a}$ is found to be:

$$
F_{a}=2 \mu_{a} \frac{r_{a}}{w_{t}} F_{t_{y}}^{i}
$$

where the factor 2 is from the two point loads on the axle and $\mu_{a}$ is the coefficient of friction between the axle and the wheel. The magnitude of $\mu_{a}$ was selected to match the 
experimental data. Also $r_{a}$ is the axle radius and $w_{t}$ is the width of the tire. The same $\mu_{a}$ and $r_{a}$ was used for the main and tail tires. The radius of the tire, $r_{t}$, cancels out.

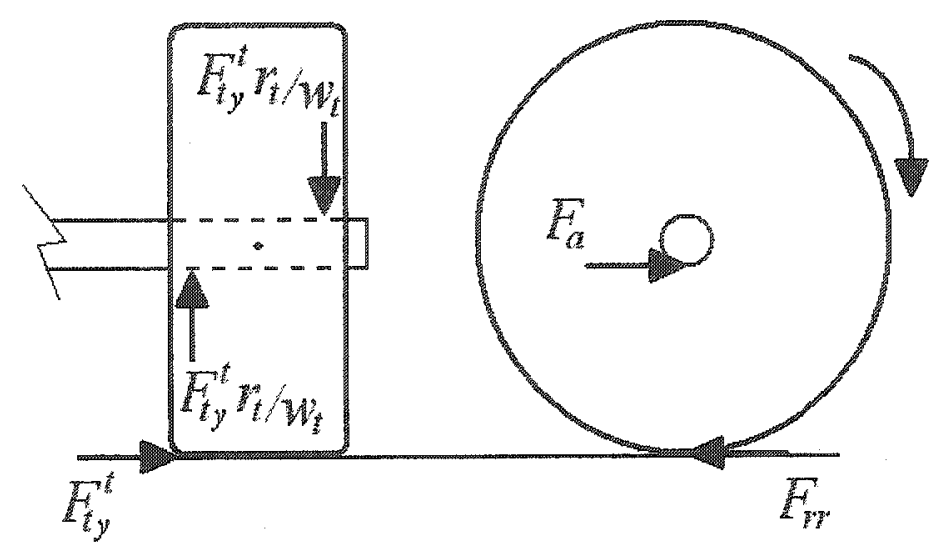

Figure 3.21: Schematic of forces acting on the tire axle

The axle friction force, $F_{a}$, was added to Equation 2.53 in Section 2.1.3, which determines the force acting on the tire in the longitudinal direction, $F_{t_{x}}^{t}$. The same smoothing function was used to prevent the force from acting when the wheel does not have a velocity.

Figure 3.22 shows the revised simulation results for the main probe force in the $\mathrm{x}$ direction with the tire axle friction added. When compared to the simulation results presented earlier in Figure 3.18, the added component has improved the accuracy of $\mathrm{He}$ liman significantly. The added component had no effect on the y component of the main probe force and the lateral tire deflections for Case 1. Therefore, the revised simulation was used next for Case 2. 


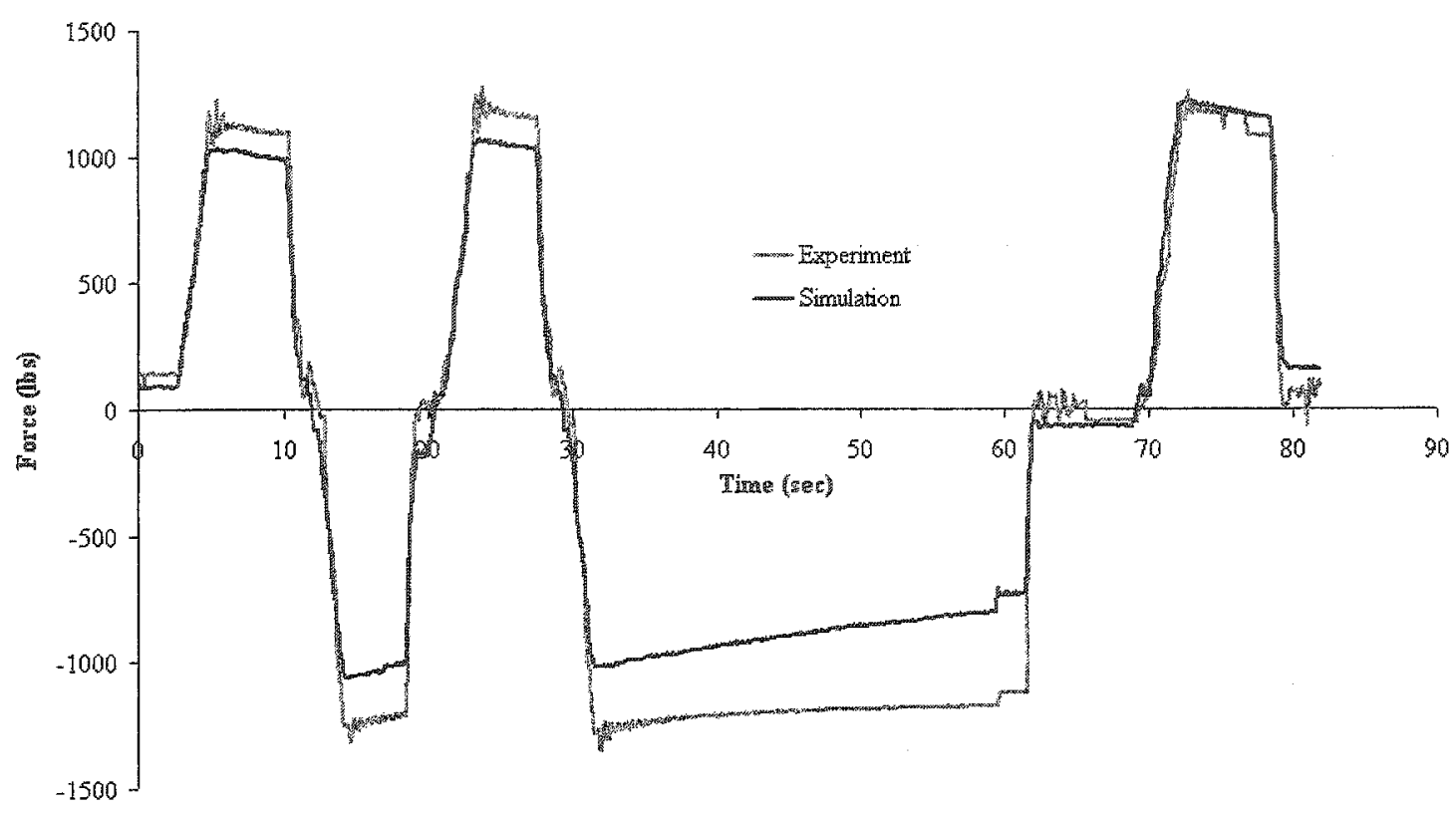

Figure 3.22: Comparison of the force acting on the probe in the DLTV's $\mathrm{x}$-direction for Case 1 with the new axle friction component

\section{Case 2: Traversing While Yawed}

For this test case the DLTV was set up as shown in Figure 3.23 with an orientation angle of $9^{\circ}$ to port. The castor assembly was also locked in the aligned position.

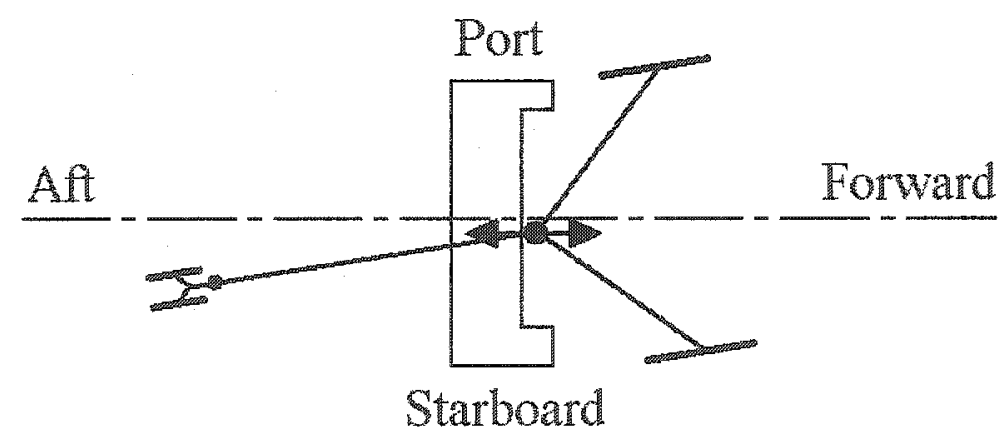

Figure 3.23: Initial position of DLTV and direction RSD is moved for Case 2

The purpose of this case was to test the yawed generation of lateral tire deflections in the main tires. The DLTV was manoeuvred forward with the given yaw angle and then manoeuvred aft to relax the generated deflections. Figures 3.24 and 3.25 show the 
experimental and simulated RSD claw positions, respectively. The simulated and measured results match very closely, so the simulation model experienced the same inputs as the DLTV.

For this case and the subsequent cases analyzed, the time at which the testing was stopped to do hand measurements has been cut out to create a continuous time history. Some minor jumps in the results are experienced because of this. A small amount of drift can be observed in the experimental lateral RSD claw position. It was found that this drifting corresponded to the points when the system was stopped to complete hand measurements. In Figure 3.25 the diamonds identify the points when the test was stopped. Also, because the inertial forces have to be overcome each time the test was stopped, bouncing occurs in all of the parameters recorded.

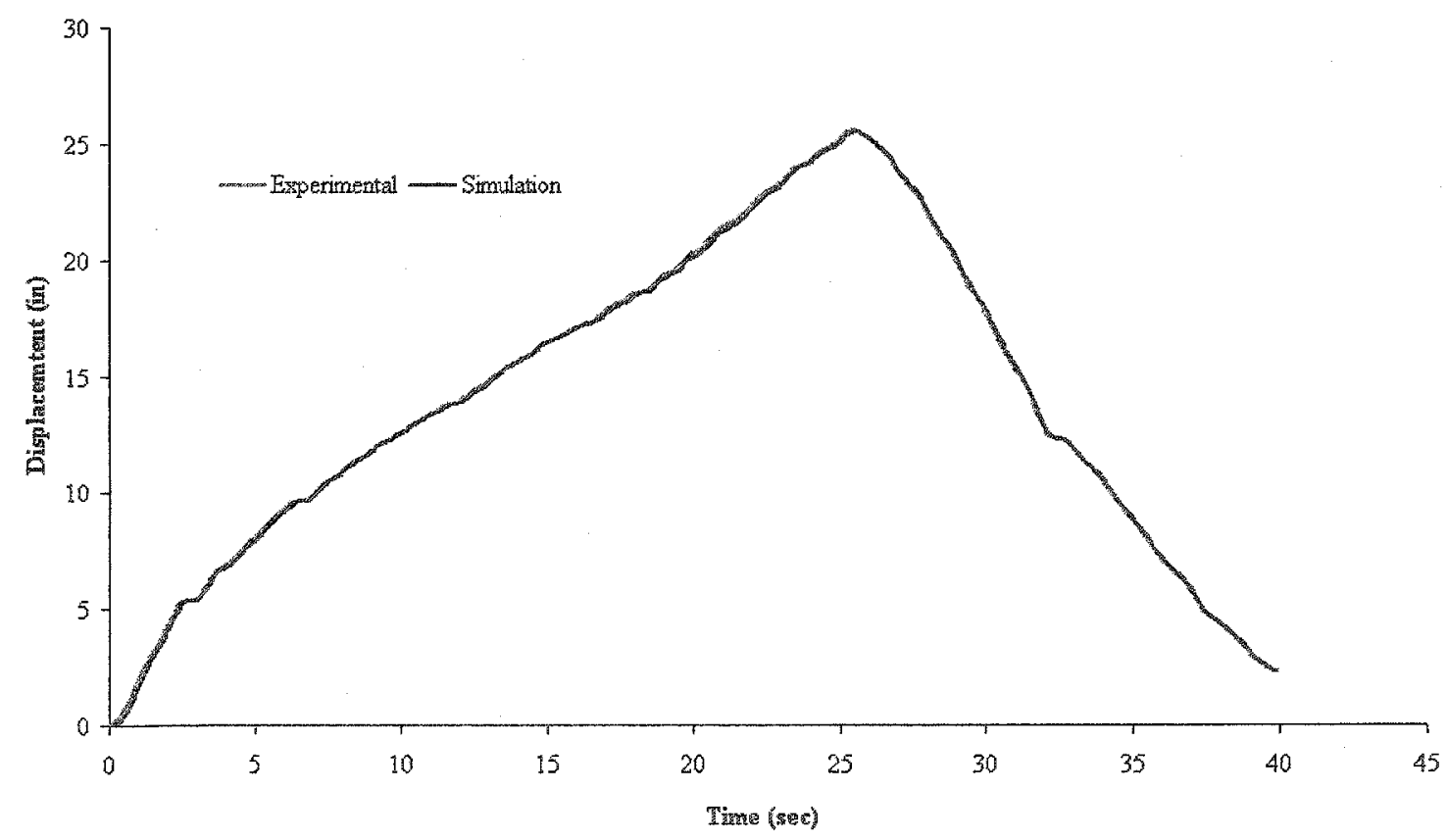

Figure 3.24: Comparison of the RSD claw x-position for Case 2 


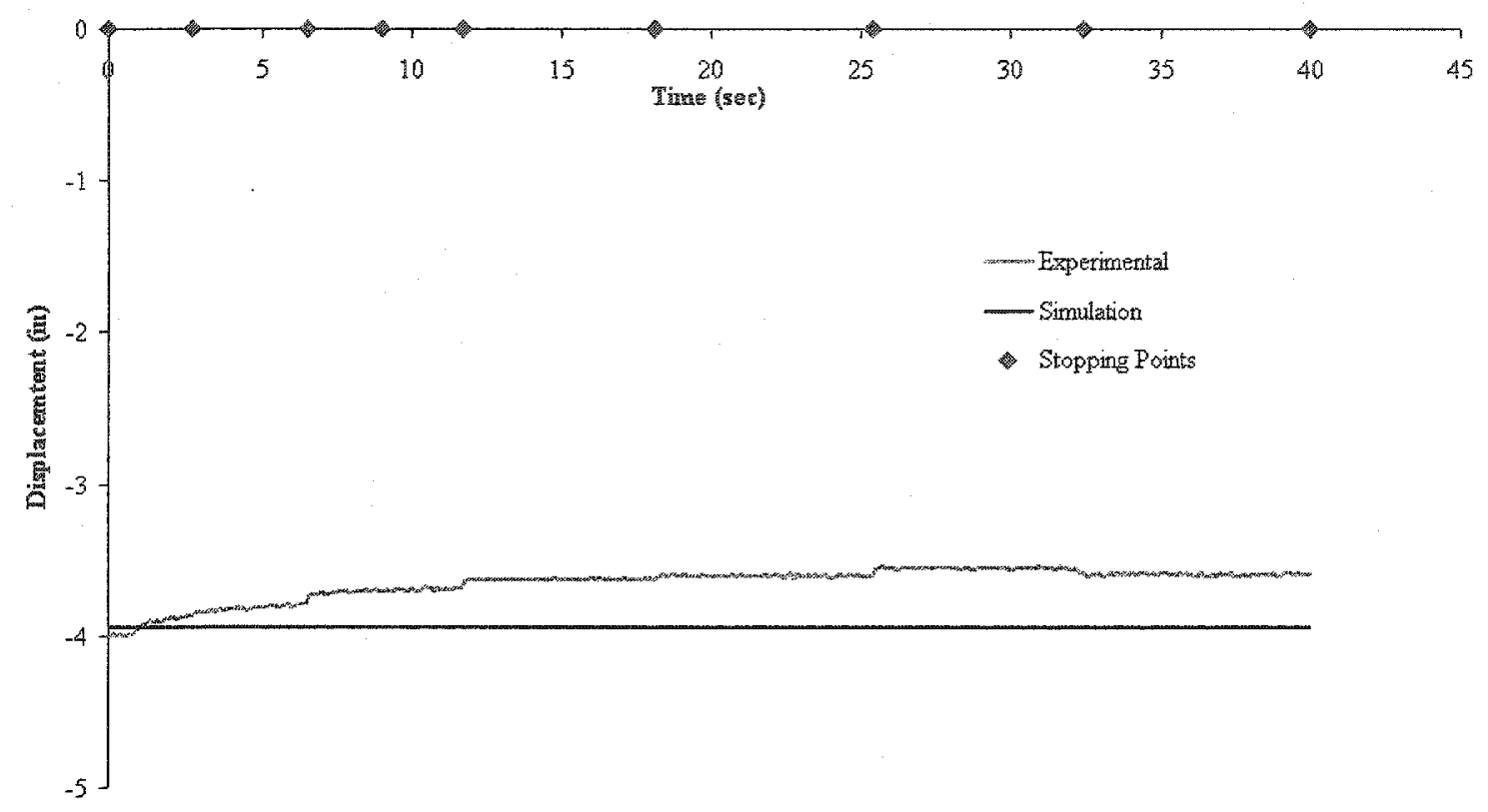

Figure 3.25: Comparison of the RSD claw y-position for Case 2

Figure 3.26 shows the plotted probe force in the $\mathrm{x}$-direction. The simulated $\mathrm{x}$ component of the main probe force corresponds well with the experimental results as the DLTV was traversed forward. However, when the DLTV was traversed aft the simulated $\mathrm{x}$ component of the force instantly switches to a negative value. This was caused by the dependence of the axle friction and rolling resistance model on the direction of the tire's velocity. The resistance forces have to oppose the direction of motion.

Another property of the axle friction force to consider is that it is a nonconservative force. The $\mathrm{x}$ component of the main probe force trend for the experimental results appear to be acting in a semi-conservative manner. This indicates the source of the problem with the $\mathrm{x}$ component of the main probe forces may relate to a spring-type behaviour. The problem could be caused by longitudinal deflections in the tires. In all of the longitudinal tire deflection models seen, including an empirical relationship derived by Smiley and Horne [34], longitudinal tire deflections only occur when brakes are applied. However in this case, the longitudinal deflections, if the cause of the discrepancy, would be a function 


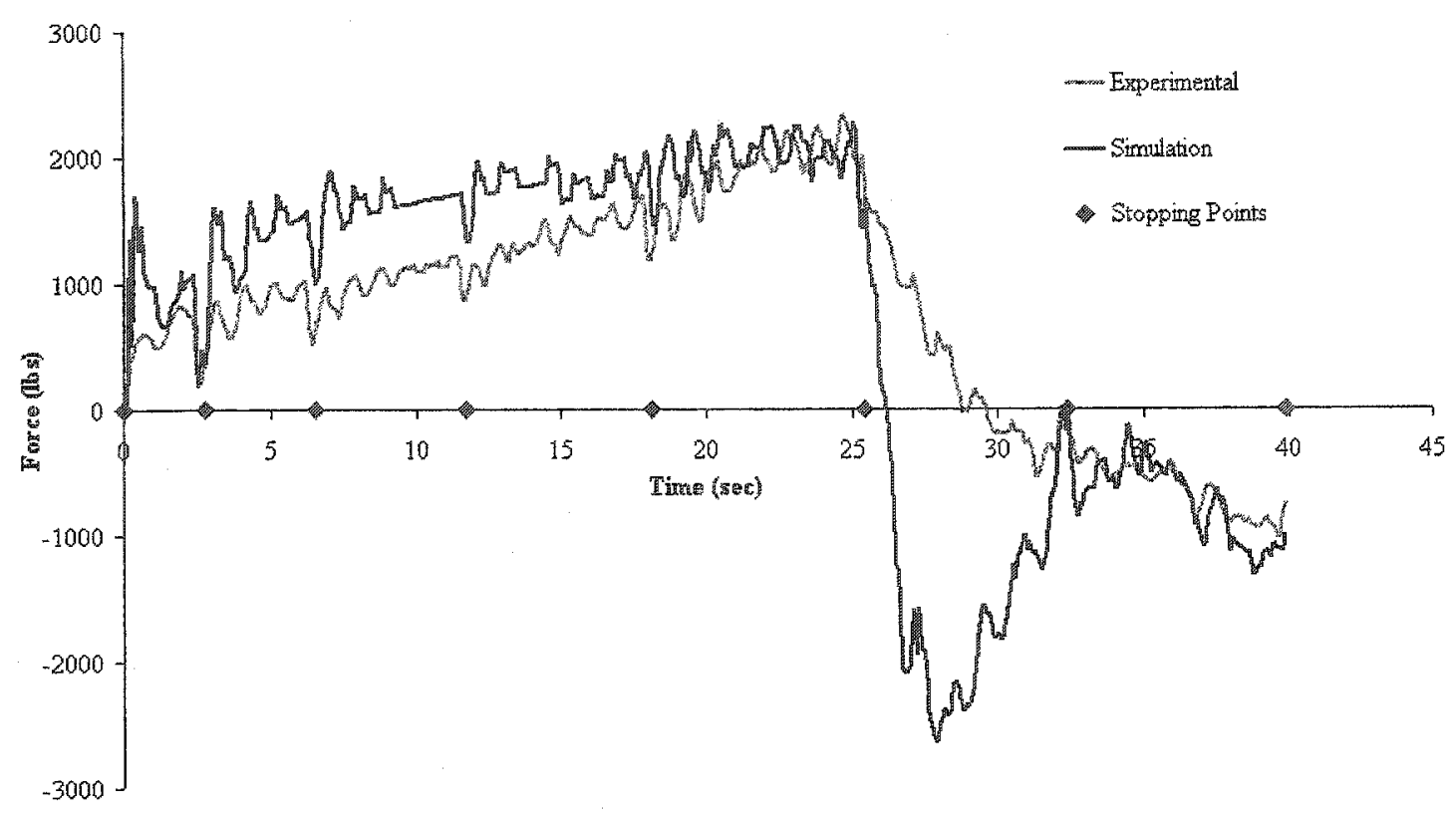

Figure 3.26: Comparison of the force acting on the probe in the DLTV's x-direction for Case 2

of the lateral force acting on the tire.

It may be possible that the axle friction component of the tire model is acting like a brake causing a longitudinal deflection. However, in most models with the brakes being applied, the tire is assumed to not roll, so at what point the model switches to the rolling model would have to be determined. The two models will most likely overlap each other. Smiley and Horne [34] have not addressed this problem. It may be that airplane wheels do not experience the direct lateral loading the helicopter wheels do under these conditions.

Another short exercise was completed to test the sensitivity of the strain gauge placement on the probe. In the practical application of the ASIST probe it is left to rotate freely. On the DLTV the probe has been pinned in place so the frame of reference is fixed. It was possible that the strain gauges may have not been applied exactly perpendicular to each other. Figure 3.28 illustrates this possibility by showing the $\mathrm{x}$ strain gauge off the axis by an angle of $\varphi$. It was found that in this configuration the $F_{x}^{\text {mea }}$ value will measure a component of the $F_{y}^{a c t}$. This added component to $F_{x}^{\text {mea }}$ is calculated by $F_{y}^{a c t} \sin \varphi$. It was 
found that to make up for the magnitudes seen in. Figure 3.26 the error in angle would have to be extremely large. ITI uses these strain gauges for factory acceptance tests and would have corrected any significant problems in the setup if this were in fact the problem.

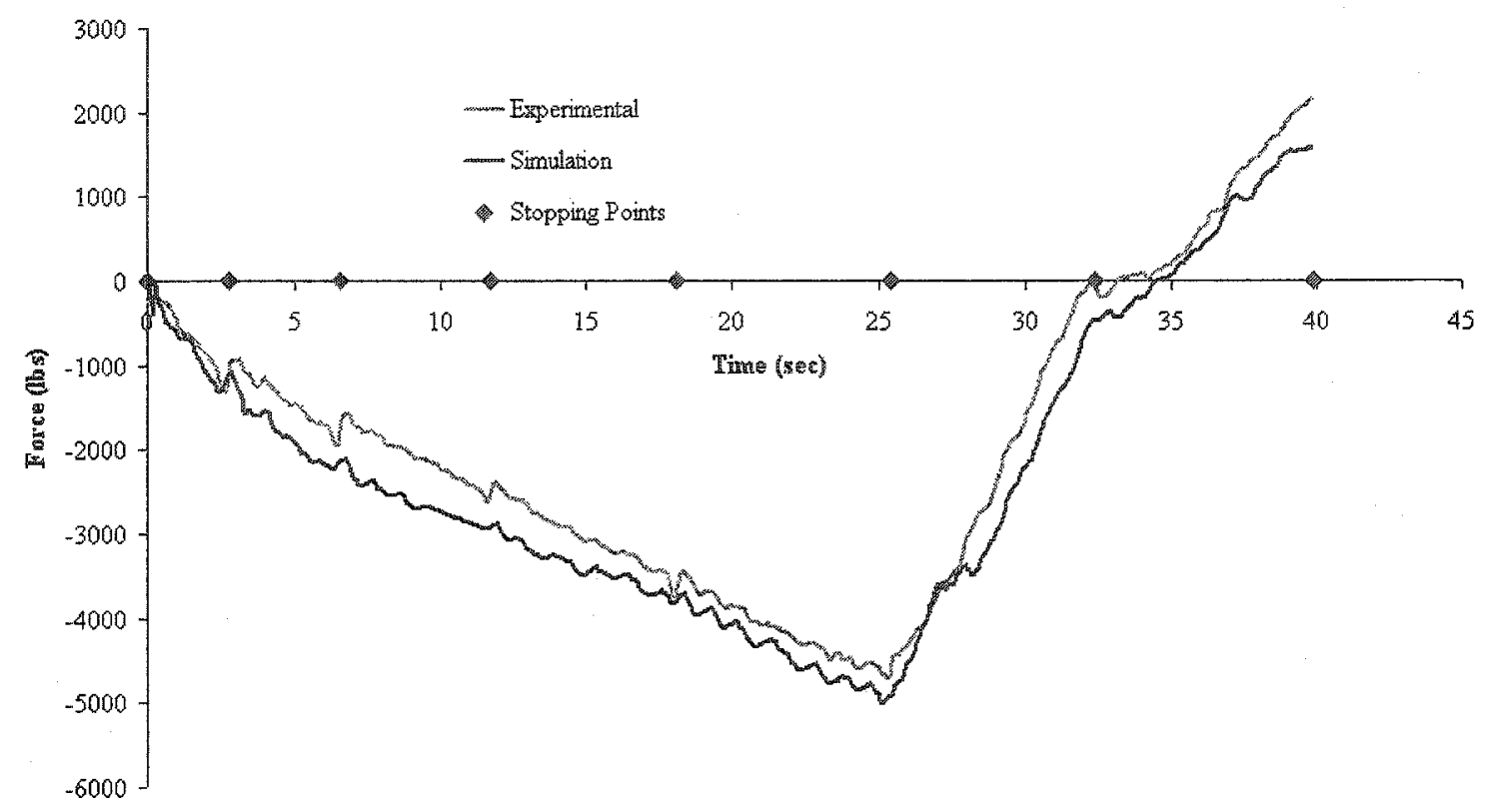

Figure 3.27: Comparison of the force acting on the probe in the DLTV's y-direction for Case 2

The simulated values for the $\mathrm{y}$ component of the main probe force correspond well with the experimental results. The comparison is plotted in Figure 3.27. Again, some bouncing occurs at the stopping points. The measured experimental force values appear to decrease slightly each time it was stopped, making subsequent values later in the test slightly smaller.

Figure 3.29 shows the lateral tire deflections for the main gear. Again, the tire model demonstrates a tendency to balance the two deflections out. In the case of the two deflections being generated, the right tire, which is initially larger, builds up slower than the initially smaller one, the left tire. In the experimental case both lateral tire deflections build up at the same rate. So by the time the DLTV was about to be manoeuvred back, 


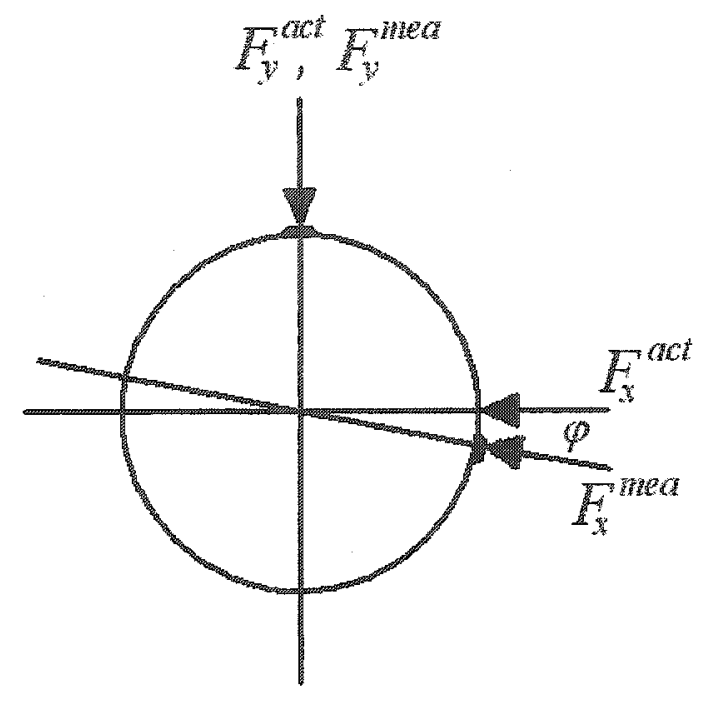

Figure 3.28: Schematic of strain gauge placement on the probe

both of the simulated values were almost identical.

It was found in early analysis of the next cases that the added axle friction model did not improve the simulation performance and at some points increased the discrepancies. As a result, for the last four test cases the axle friction model was left out. 


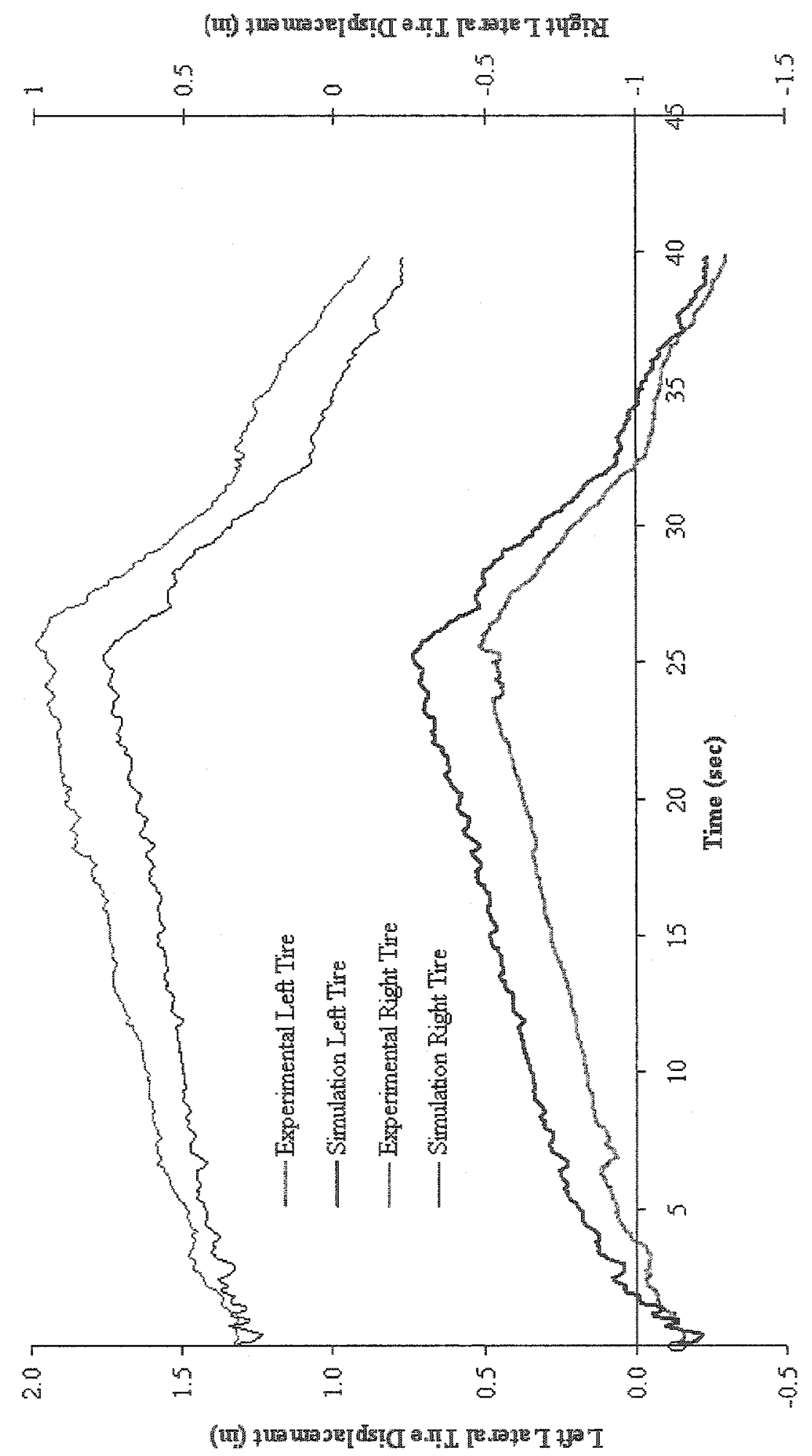

Figure 3.29: Comparison of the lateral tire deflections for Case 2 


\section{Case 3: Yawed to 90 Degrees}

For this test case the DLTV was set up as shown in Figure 3.30 with an orientation angle of $9^{\circ}$ to port. The castor assembly was free to rotate, but started in the aligned position.

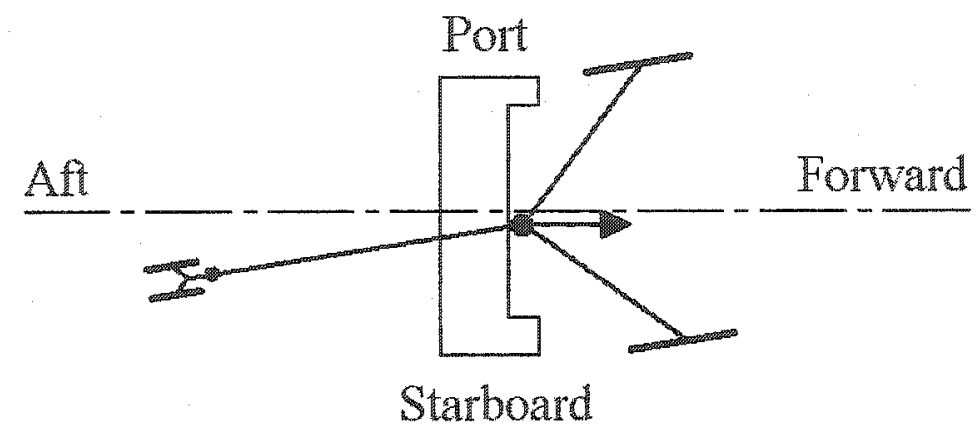

Figure 3.30: Initial position of DLTV and direction RSD is moved for Case 3

The purpose of this case was to test the yawing tendency of the Seahawk landing gear configuration when traversing forward. The DLTV was traversed forward while the tail was allowed to swing freely in the starboard direction. The RSD claw position was assumed to remain constant in the lateral direction. The longitudinal and lateral RSD claw positions are plotted in Figures 3.31 and 3.32 respectively. Some minor drifting occured in the lateral direction.

The DLTV and castor orientations are plotted in Figures 3.33 and 3.34. The experimental DLTV orientation was measured at discrete times by hand with the test stopped. The simulated orientations correspond very well with the experimental results.

Figures 3.35 and 3.36 show the plotted simulation results for the forces acting on the main probe. The simulated $\mathrm{x}$ and $\mathrm{y}$ components of the main probe forces follow the general trend of the experimental results, but their accuracy is compromised by the tire model limitations discussed in the previous two cases. Although the limitations are not as obvious in this case, they may be harder to observe due to the slightly more complex manoeuvre. The choppiness in the $\mathrm{x}$ component of the main probe force is caused by the starting and 
stopping required for manual measurements and amplified by the difference in the mass moment of inertia. The effect of the mass moment of inertia is discussed at the end of this case.

Figure 3.37 shows the plotted simulation results of the lateral deflections on the main tires. Again, they follow the general trend of the experimental data, but the accuracy is compromised by the limitations discussed in the previous two cases. The simulated lateral deflection of the right main tire does not increase as rapidly as the experimental results shifting the subsequent values lower. After this initial shift, the simulated values follow the same trend as the experimental results very well up to approximately the 60 second point. This could possibly be caused by the unknown initial conditions acting on the castor assembly.

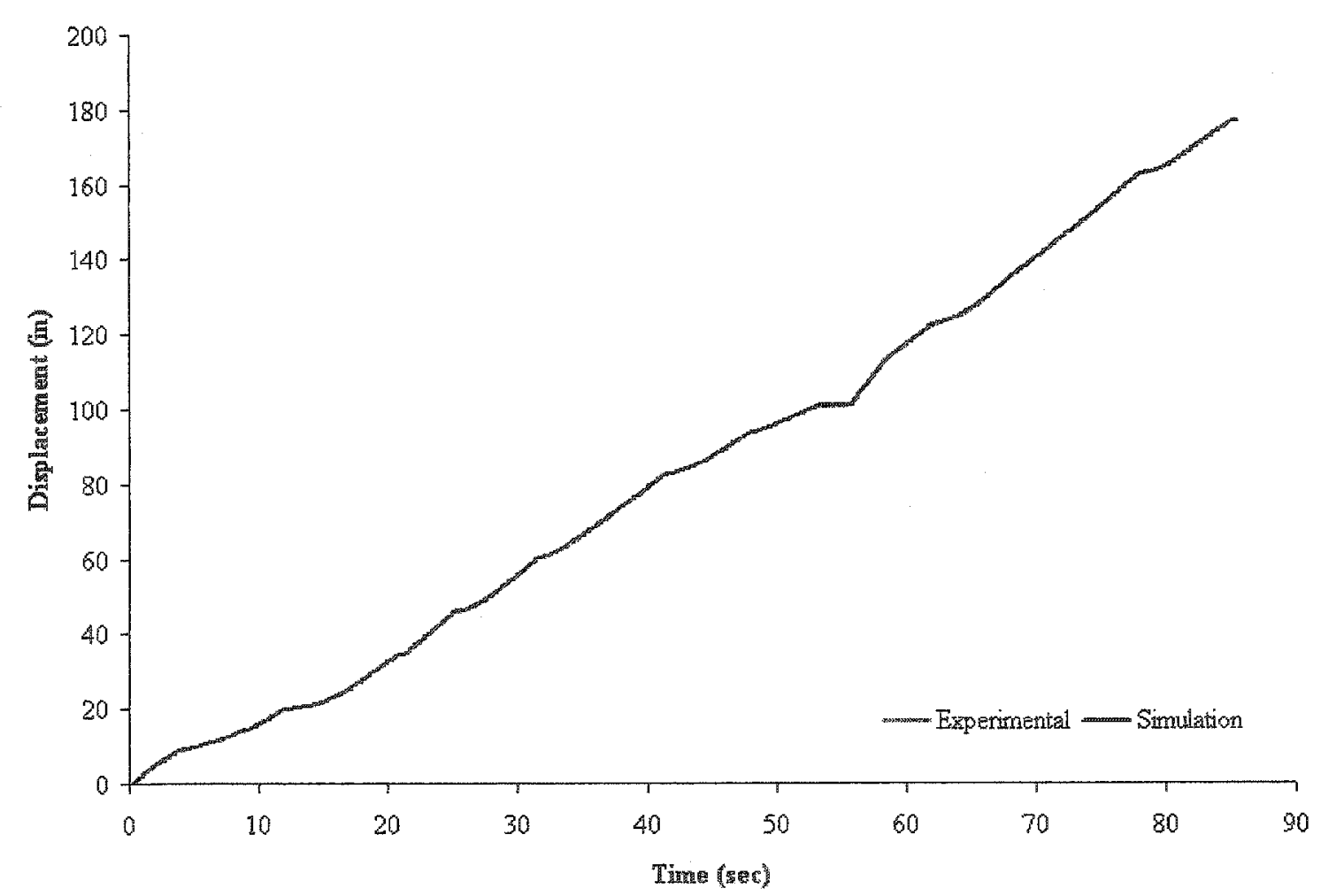

Figure 3.31: Comparison of the RSD claw x-position for Case 3 


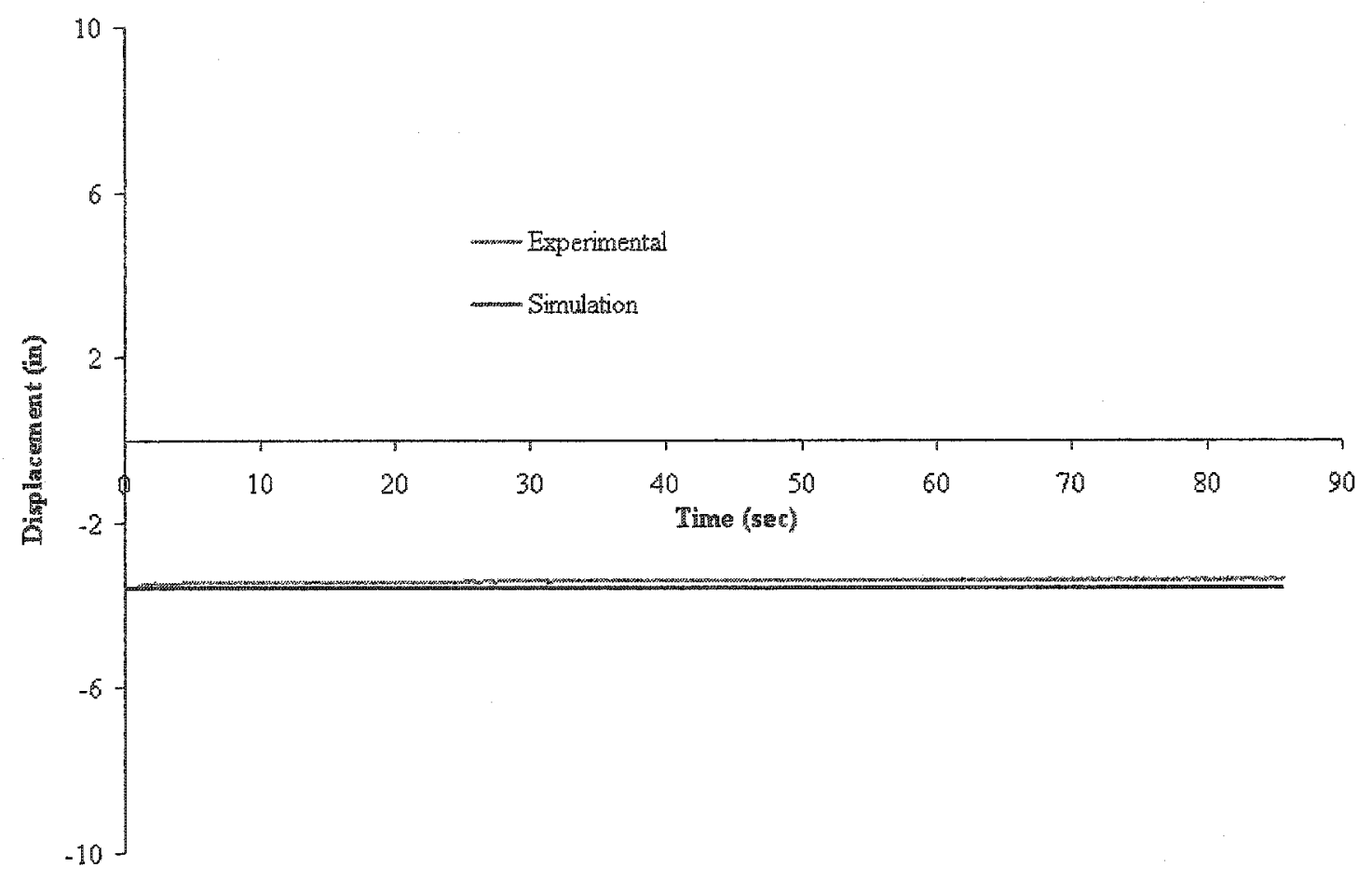

Figure 3.32: Comparison of the RSD claw y-position for Case 3

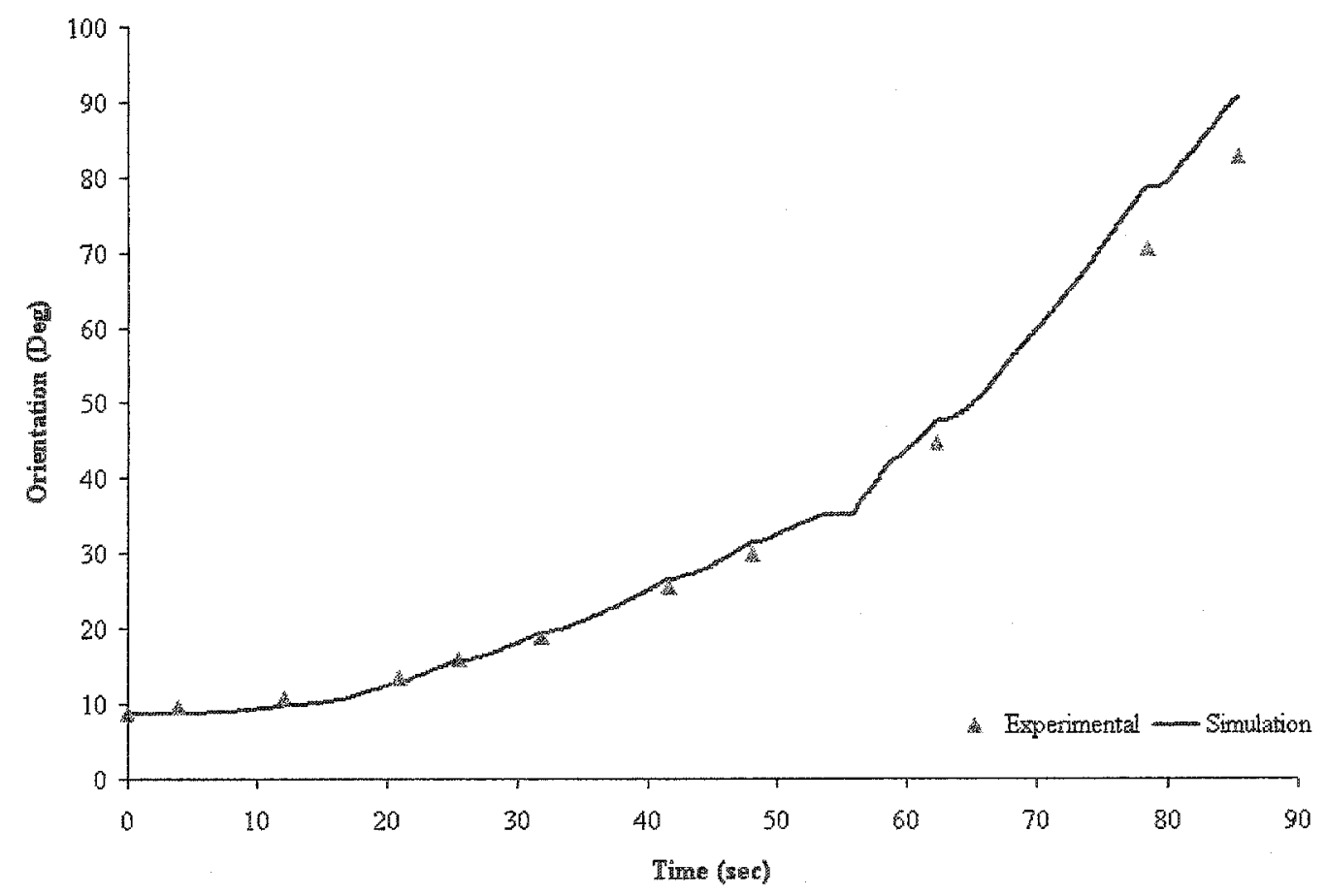

Figure 3.33: Comparison of DLTV orientation for Case 3 


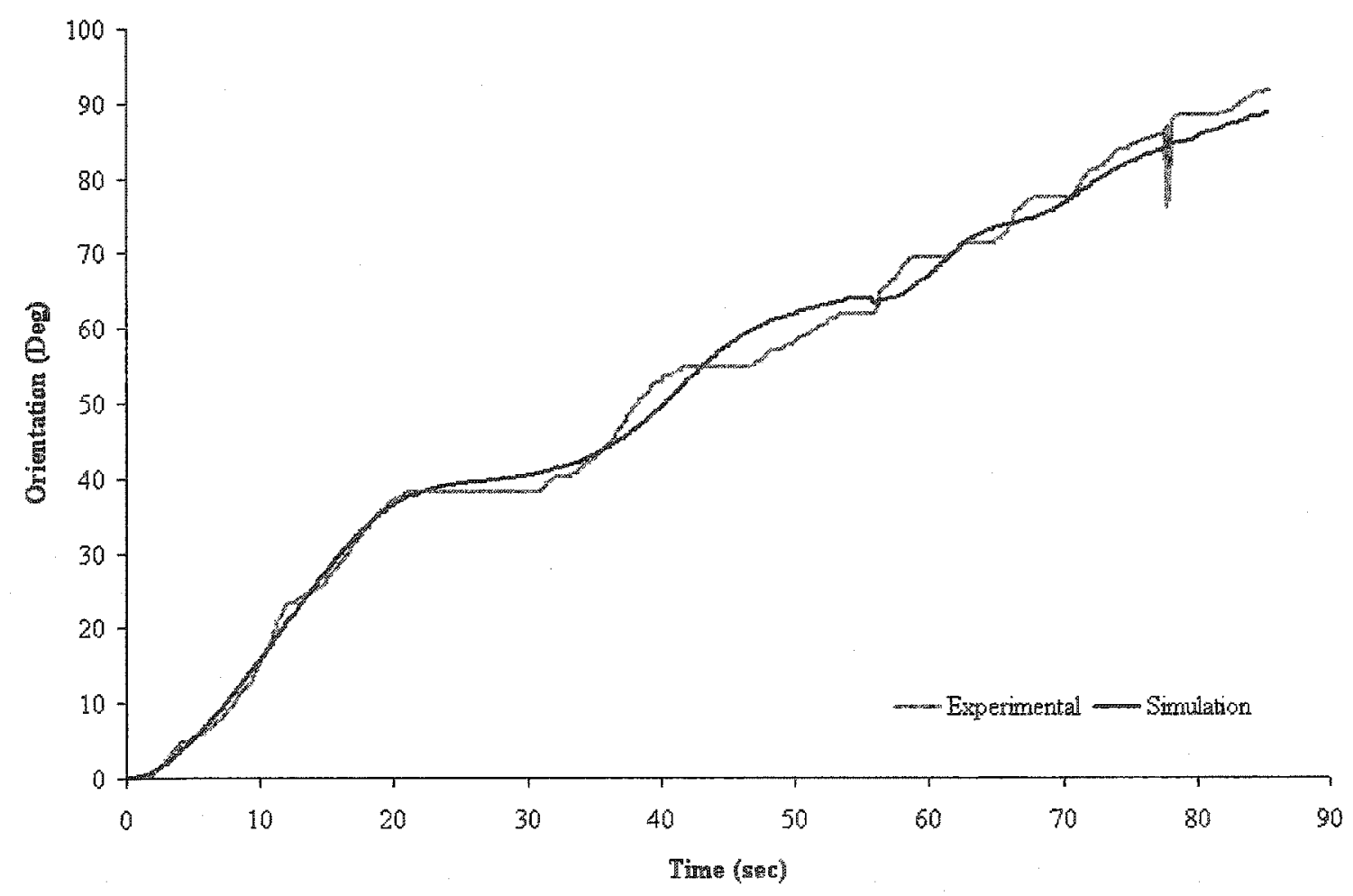

Figure 3.34: Comparison of castor orientation for Case 3 


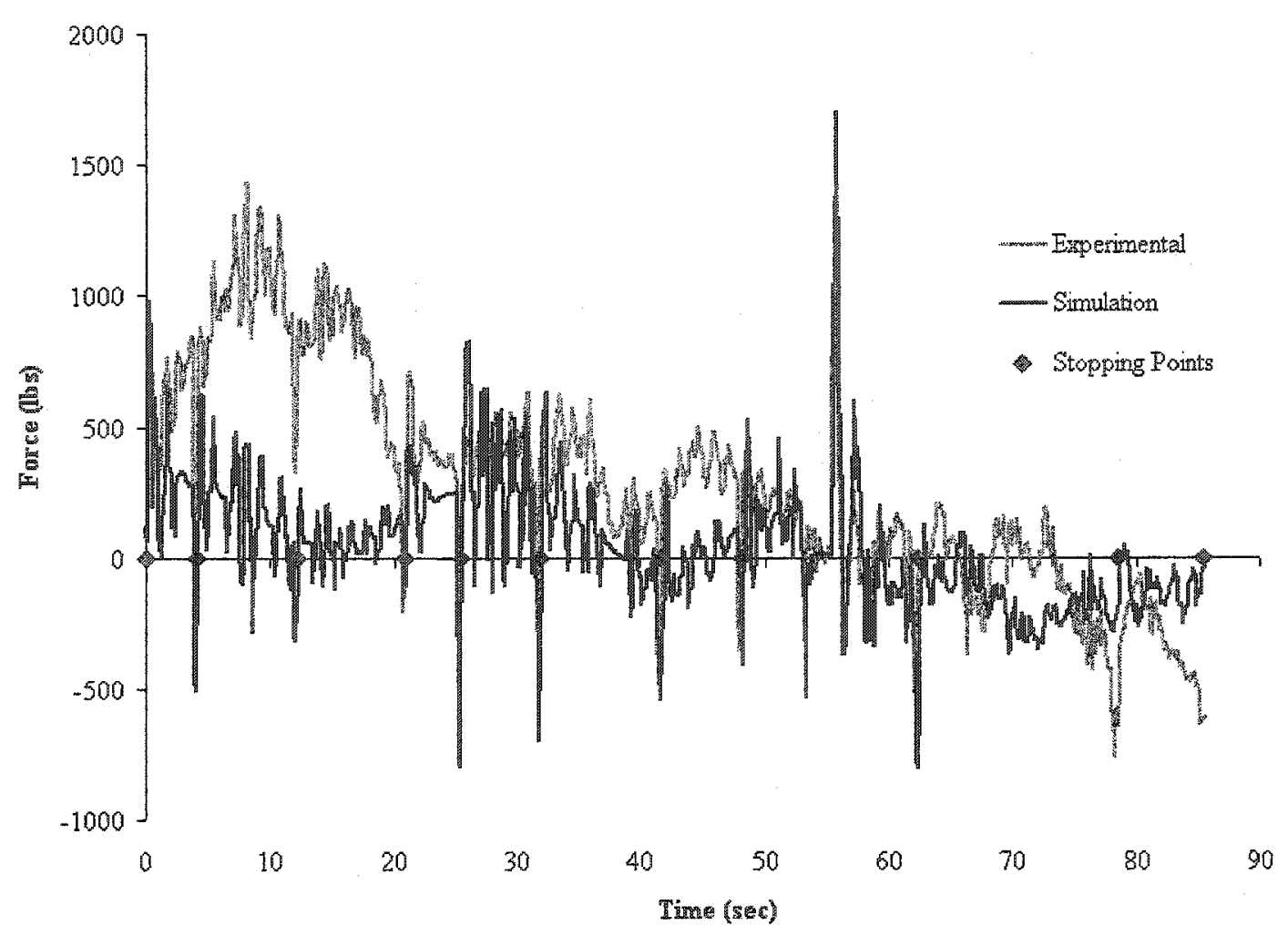

Figure 3.35: Comparison of the force acting on the main probe in the DLTV's $\mathrm{x}$-direction for Case 3

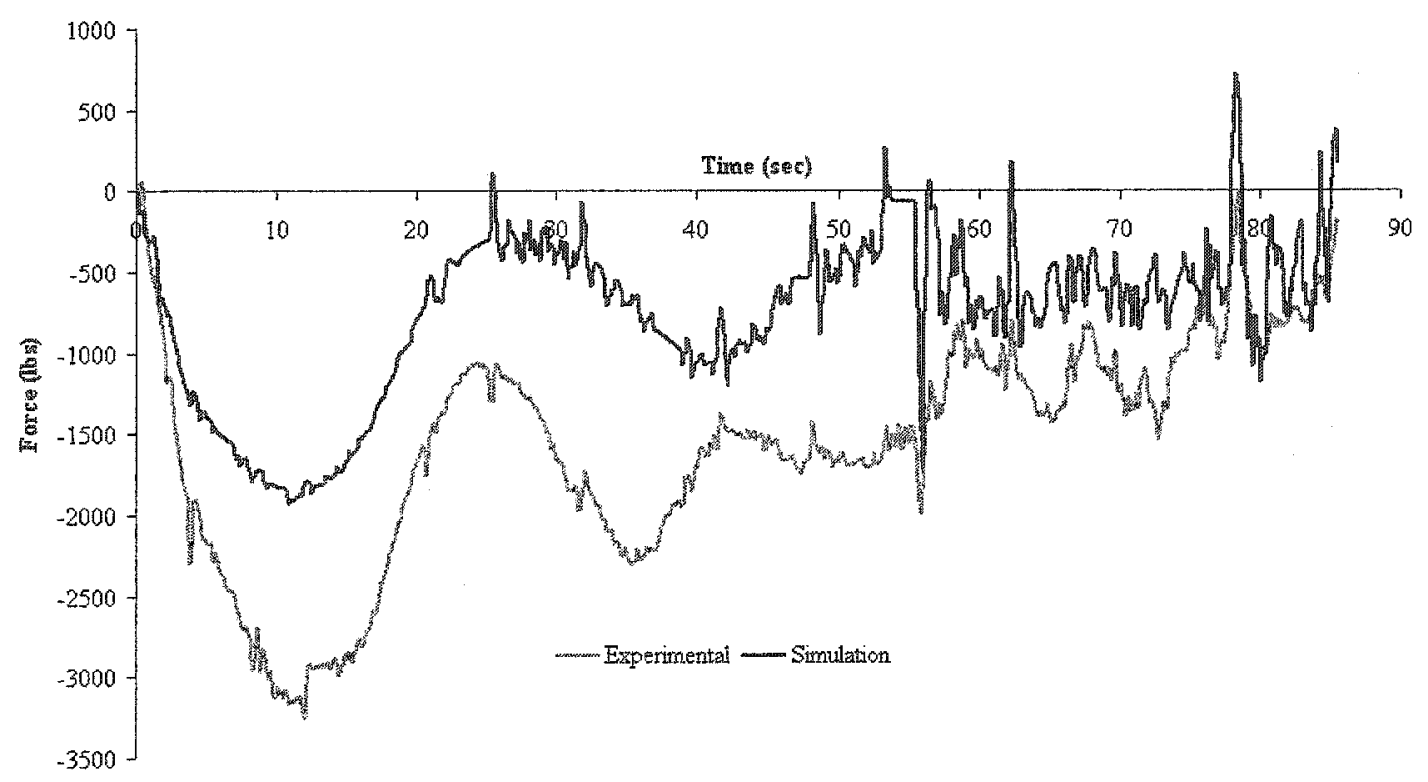

Figure 3.36: Comparison of the force acting on the main probe in the DLTV's $y$-direction for Case 3 


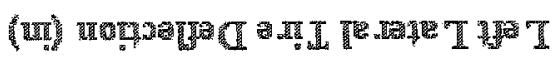

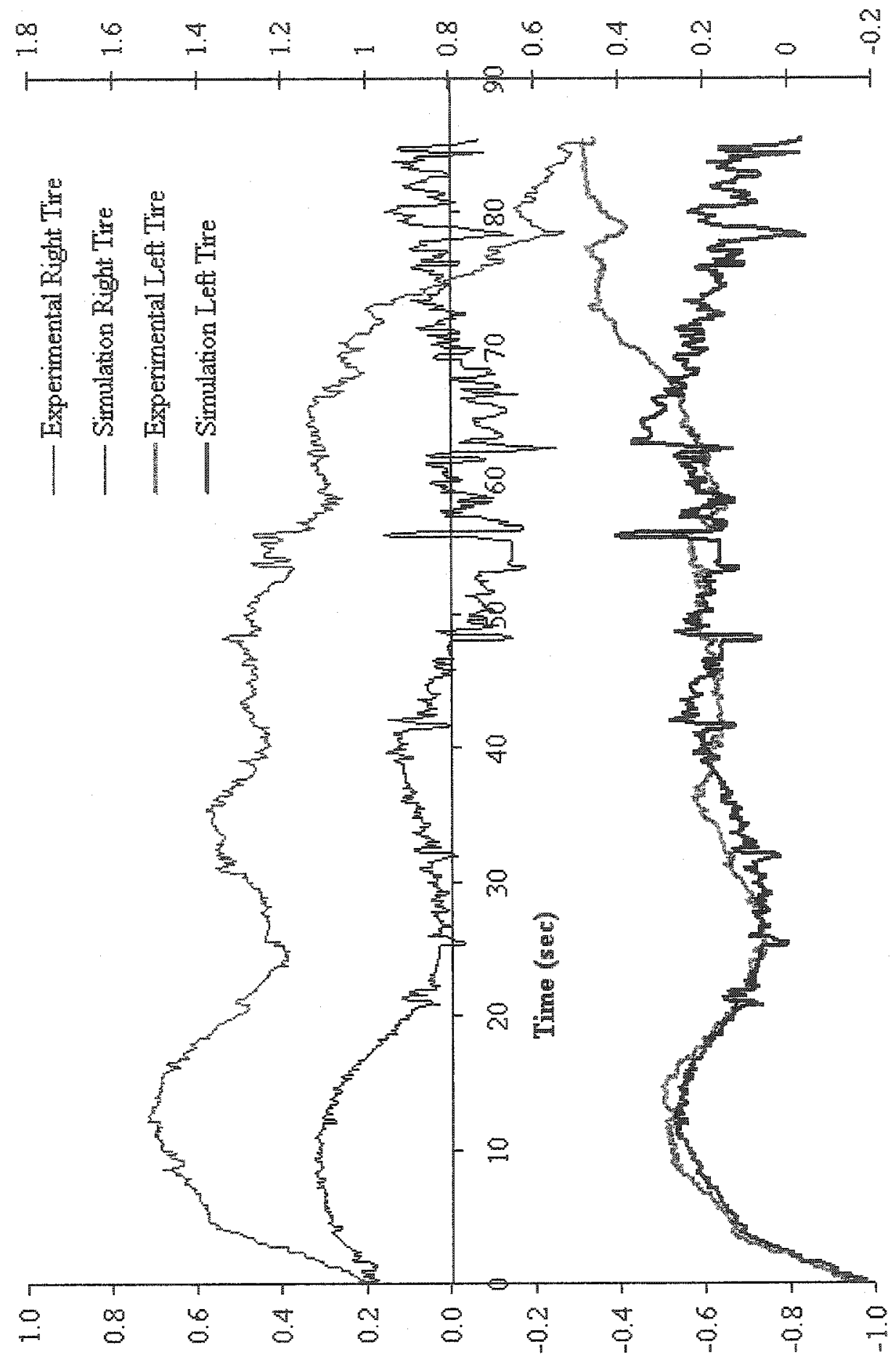

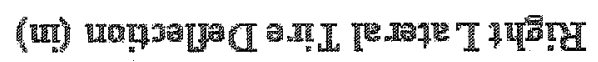

Figure 3.37: Comparison of the lateral tire deflections for Case 3 
A quick mass moment of inertia test was done using Case 3 to help demonstrate what was in Section 3.2.3. In this particular situation tested a great deal of rotational motion occurs so it will be affected more by the difference in the mass moment of inertia. Figure 3.38 shows the $\mathrm{x}$ component of the main probe force for Case 3 generated by HeliMan using the full mass moment of inertia and also using ten percent of the full value. It is observed that the smaller mass moment of inertia makes the magnitude of the spikes smaller. The spikes are caused by the frequent starting and stopping and will never go away completely unless a continuous test is performed. These spikes occur in the experimental data as well.

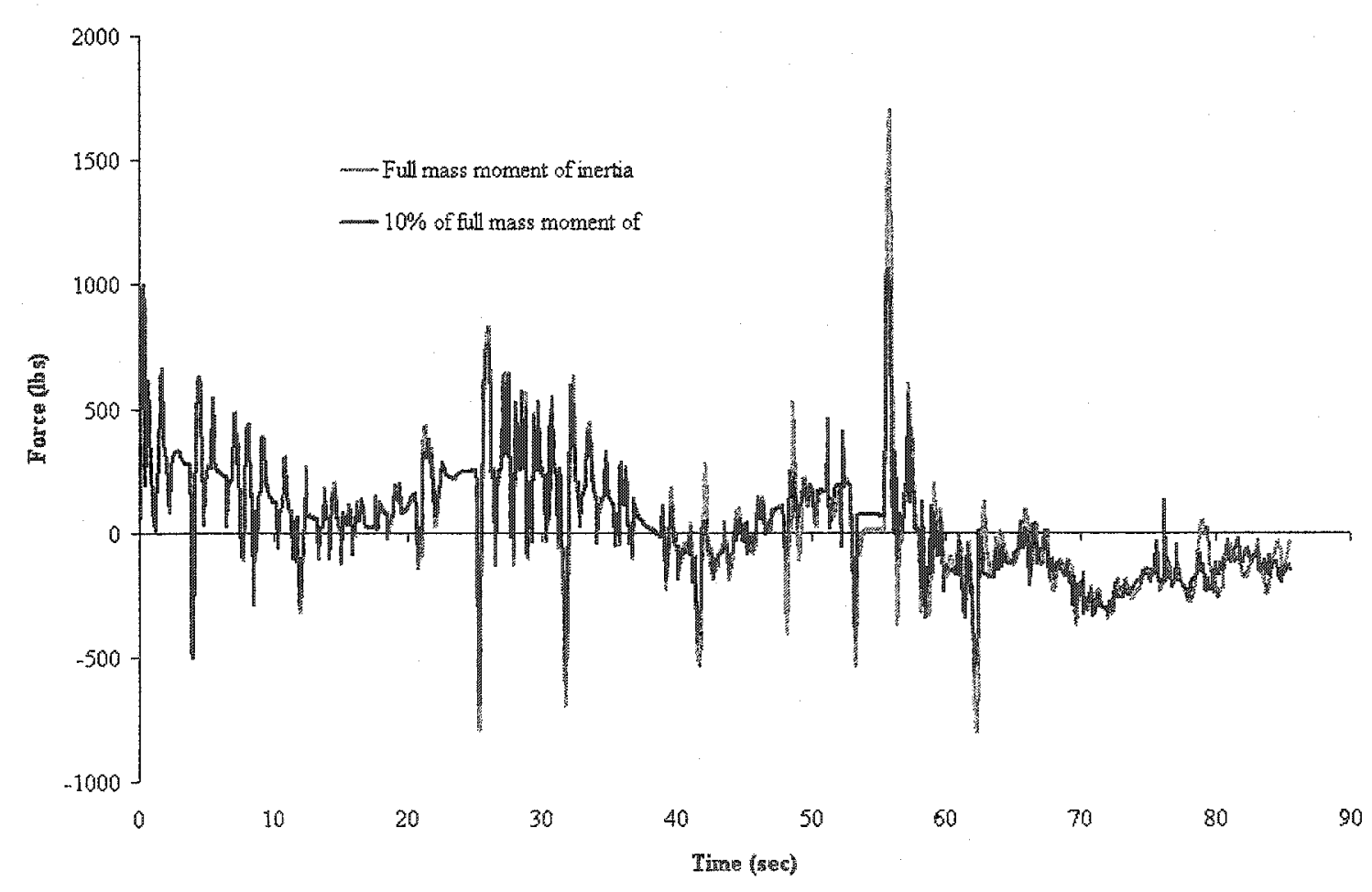

Figure 3.38: Comparison of simulated $x$ component of the main probe forces resulting from Case 3 for two different mass moments of inertia 


\section{Case 4: Yawed Back to 0 Degrees with Free Float}

For this test case the DLTV was set up as shown in Figure 3.39 with an orientation angle of $90^{\circ}$ to port. The castor assembly was free to rotate and started $-90^{\circ}$ from the aligned position.

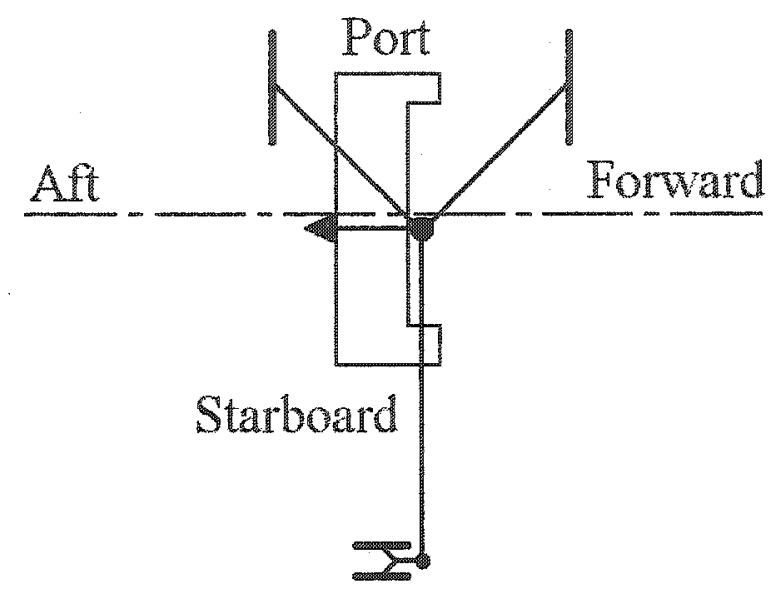

Figure 3.39: Initial position of DLTV and direction RSD is moved for Case 4

The purpose of this case is to test the straightening tendency of the Seahawk landing gear configuration when traversed aft from a large orientation angle. The free float function was used to realign the DLTV with the centreline from the orientation it reached in Case 3. The DLTV was traversed aft while the RSD claw could float laterally. As indicated previously, since the use of the float function was not recorded, the RSD claw velocities were used directly in the simulation. Figures 3.40 and 3.41 show the simulated RSD claw positions, which match closely with the experimental results.

Intuitively the castor should swivel around roughly $180^{\circ}$ from the starting orientation shown in Figure 3.39 at some point during this test. However, during the experiment the castor did not swivel around. From discussions with an experienced ASIST operator it was determined that given the right circumstances this swivelling may or may not occur. The one thing that was understood is that it is not good for the tires to allow the castor to swivel on its own in this manner. 
The results for the simulated castor orientation are shown in Figure 3.42. HeliMan ha the castor assembly swivel $180^{\circ}$ early in the test. The simulated orientation of the DLTV corresponds well with experimental results shown in Figure 3.43 despite the swivelling of the castor assembly.

It can be seen in the results for the probe forces and lateral main tire deflections in Figures 3.44 to 3.46 why it is not recommended to allow the castor to swivel. During the castor swivelling action, between the 10 and 20 second marks, a large amount of force needs to be applied to the probe to continue moving the DLTV. The main tires actually start sliding at the time corresponding to the flat part of the lateral tire deflection plot.

It must be noted that the maximum force the ASIST system can apply had to be increased in HeliMan or the RSD positions would not have corresponded with the experimental results. Otherwise the maximum force not have allowed the RSD to move in certain situations. Therefore, the maximum probe force would have peaked at the maximum value that could be applied and the main tires would not have slid. This situation is still not recommended.

After the castor swiveled the simulation results are consistent with the limitations discussed in the first two cases. The general trends seem to correspond with the experimental results, although the values differ. 


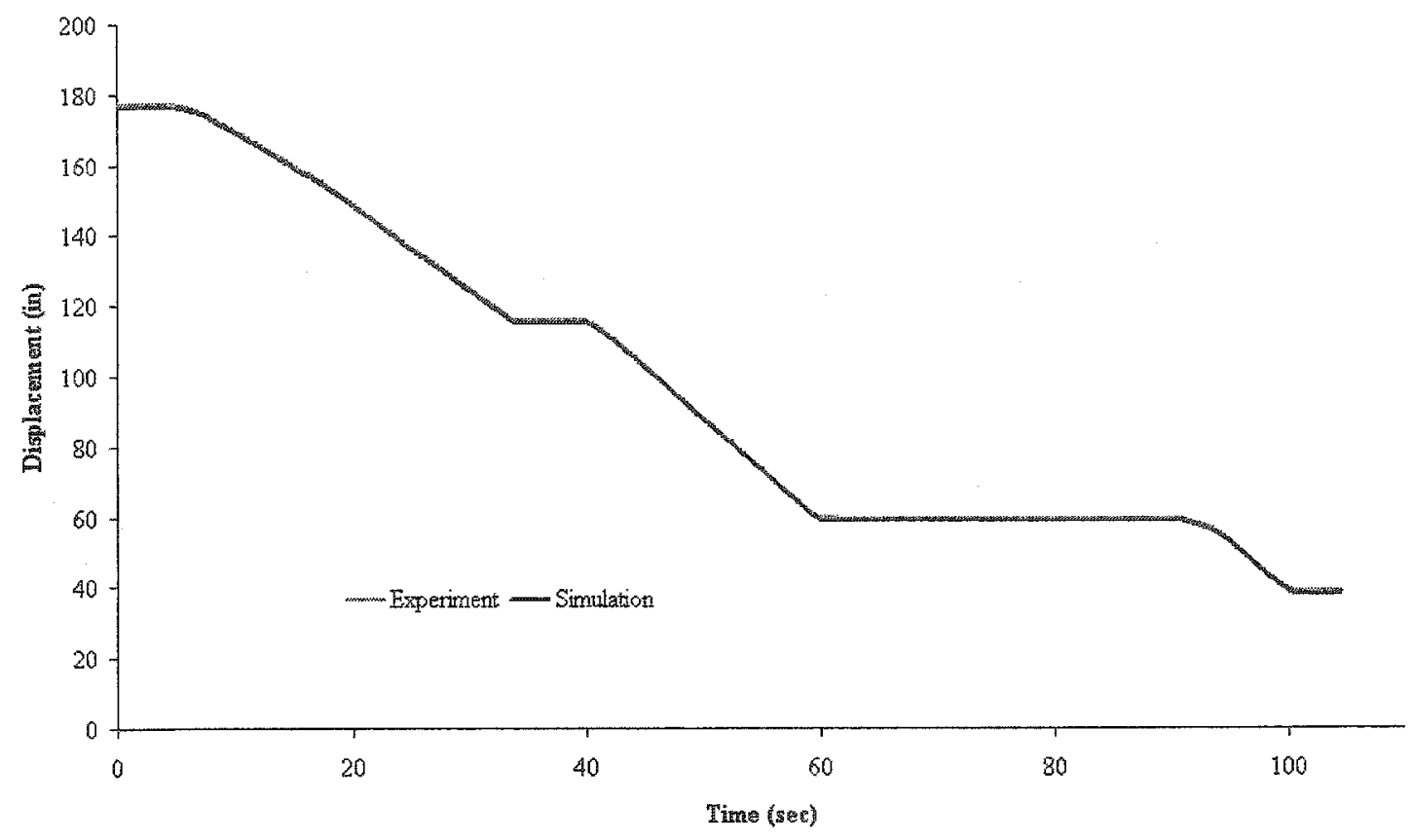

Figure 3.40: Comparison of the RSD claw x-position for Case 4

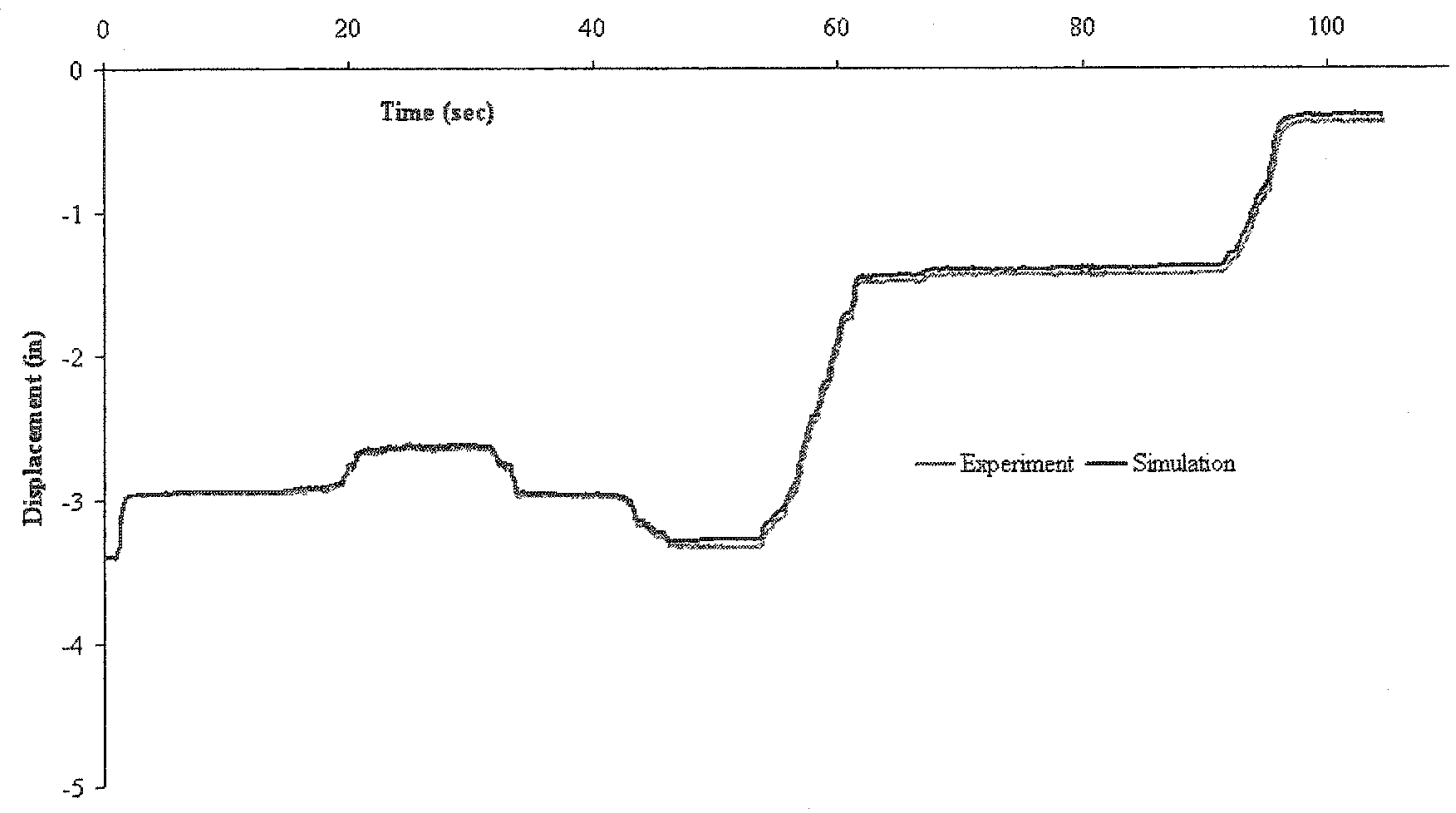

Figure 3.41: Comparison of the RSD claw y-position for Case 4 


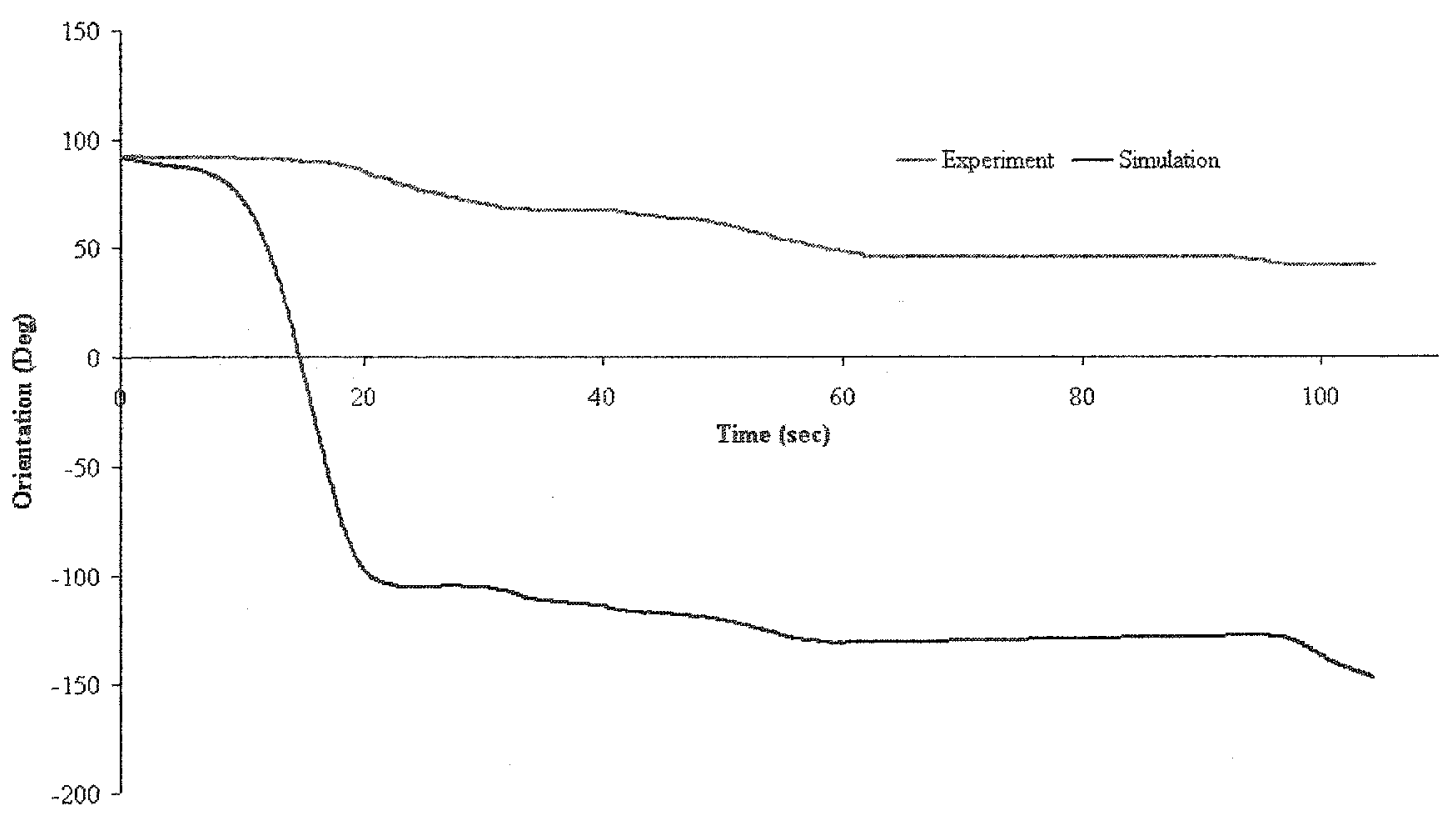

Figure 3.42: Comparison of castor orientation for Case 4

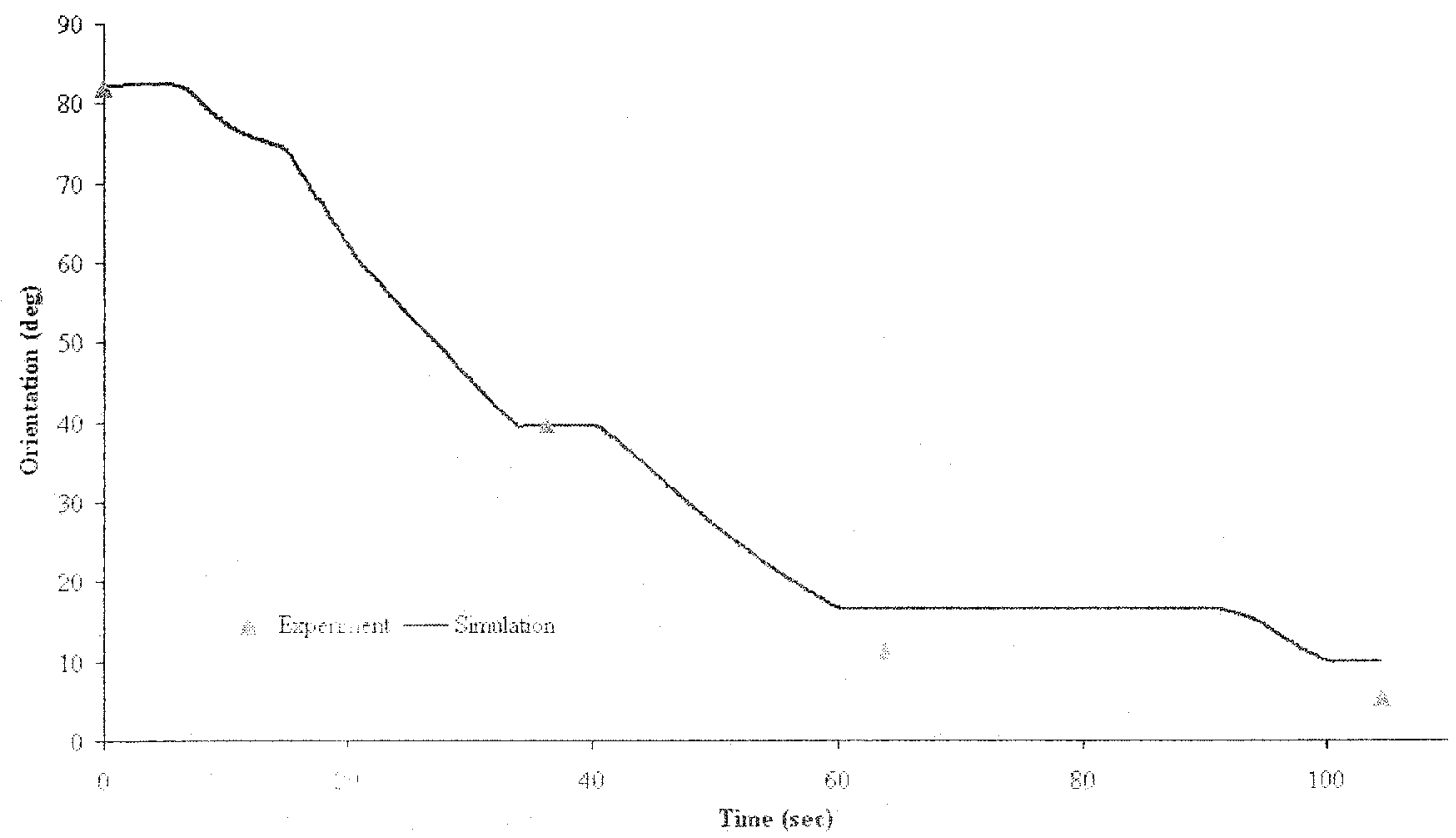

Figmre 3.43: Comparison of DLTV orientation for Case 4 


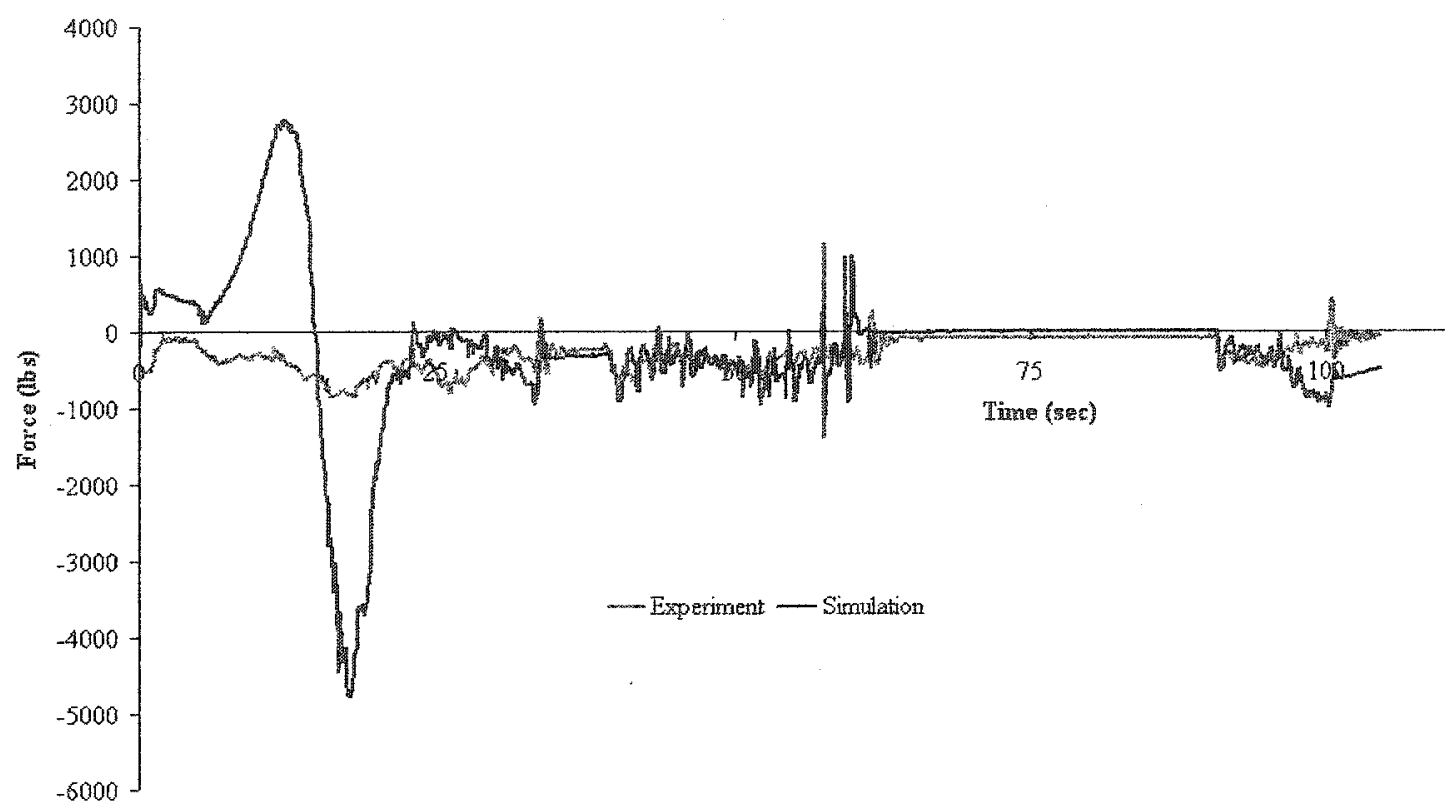

Figure 3.44: Comparison of the force acting on the main probe in the DLTV's $\mathrm{x}$-direction for Case 4

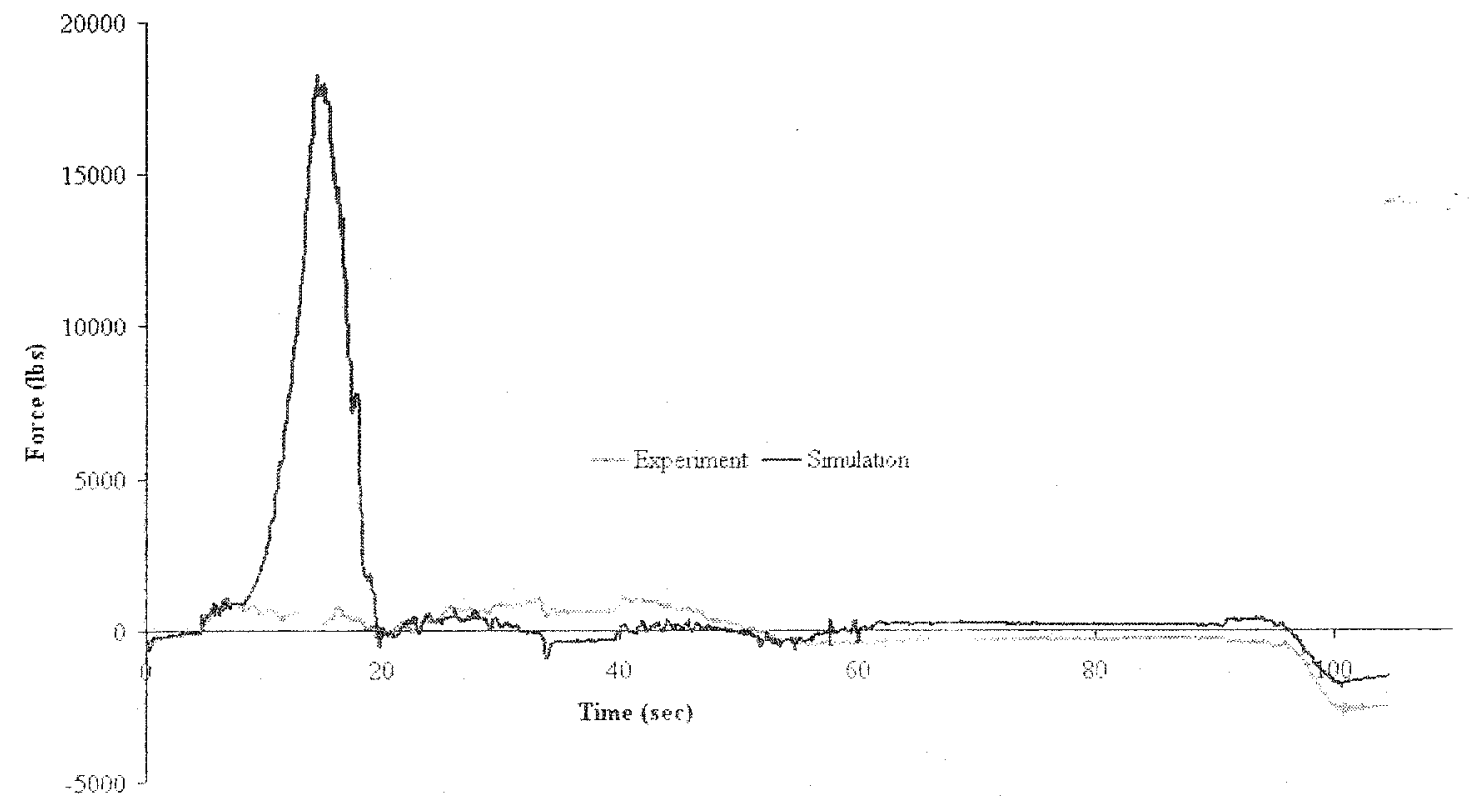

Figure 3.45: Comparison of the foren acting on the main probe in the DLTV's v-rlieretion for Case 1 


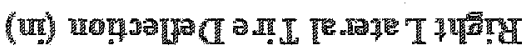

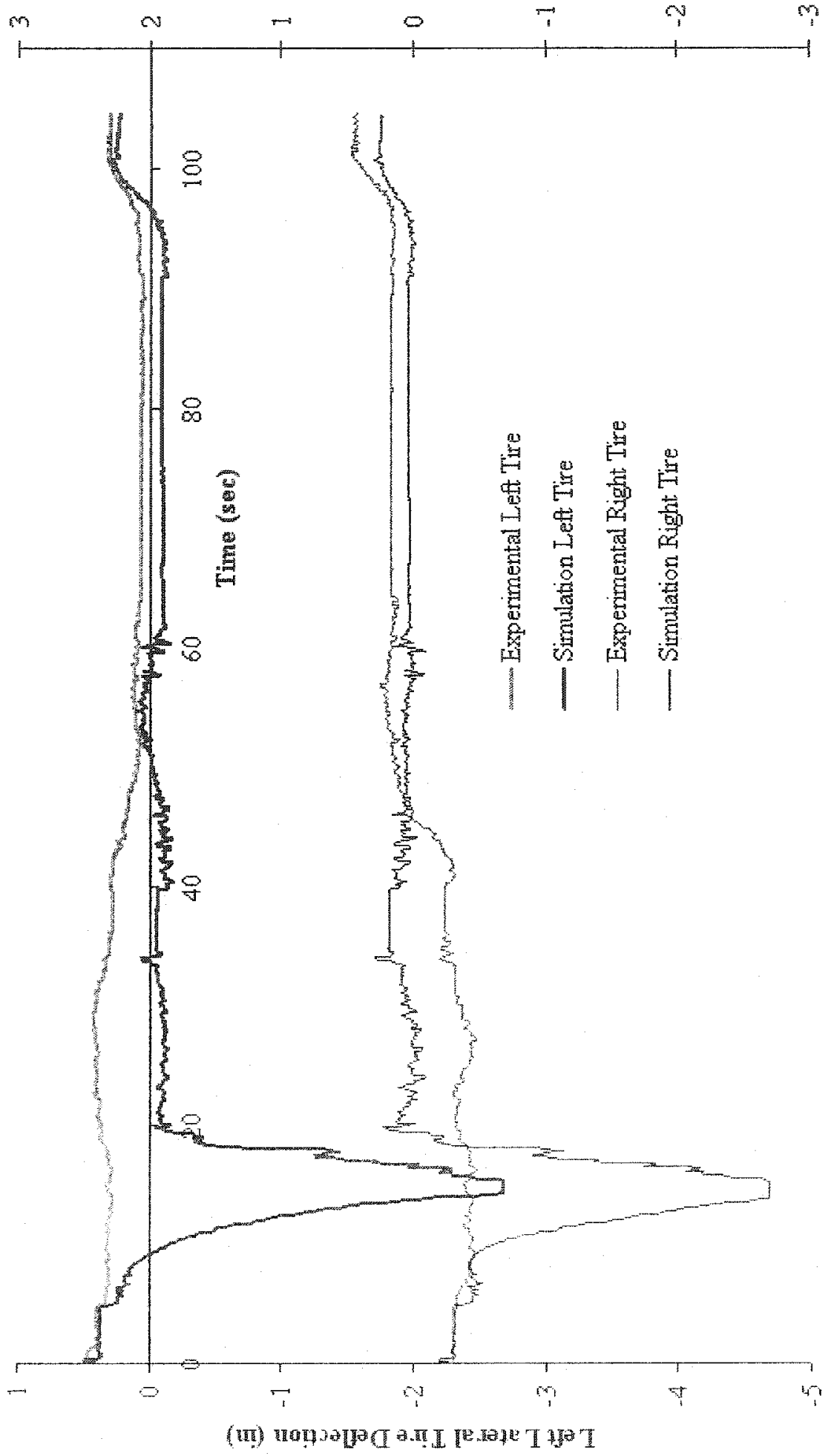

Figure 3.46: Comparion of the lateral tire deftections for Case 4 


\section{Case 5: Rotating Castor 180 Degrees}

For this test case the DLTV was set up as shown in Figure 3.47 with an orientation angle of $10^{\circ}$ to starboard. The castor assembly was free to rotate, but initially was in the aligned position.

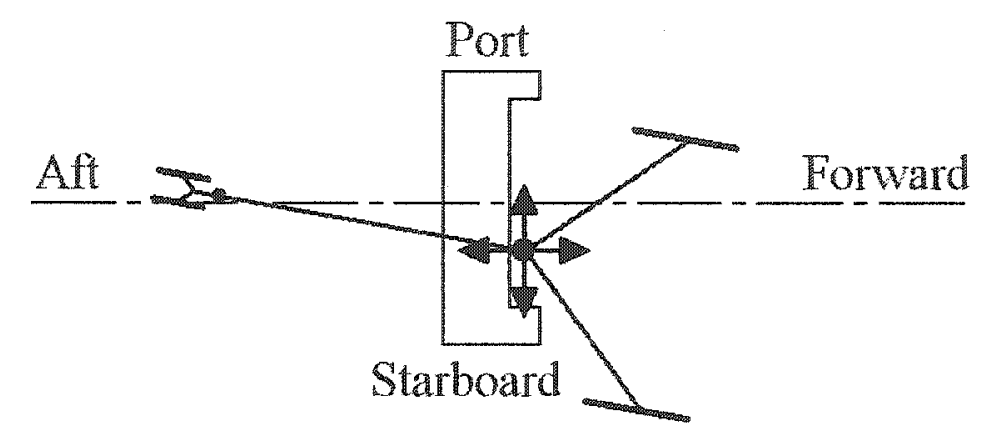

Figure 3.47: Initial position of DLTV and direction RSD is moved for Case 5

The purpose of this case was to test the castor assembly component of HeliMan. The RSD claw was manipulated so that the castor wheel swiveled around $180^{\circ}$. This was limited to $180^{\circ}$ because the castor orientation sensor would be damaged beyond $180^{\circ}$. This type of manoeuvre would be performed in service to prevent what was experienced in Case 4. Figures 3.48 and 3.49 show the positions of the RSD claw during the manoeuvre. The experimental RSD position values match the values used in the simulation.

The DLTV and castor orientations are plotted in Figures 3.50 and 3.51. The experimental DLTV orientation was neasured at discrete times by hand with the test stopped. The simulated orientations correspond very well with the experimental results. The simulation consistently predicts the orientation of the DLTV and the castor angle. Althongh some differences are observed in Figure 3.51.

Figures 3.52 to 3.53 show the plotted simulation results for the finces acting on the main probe and the lateral deflections of the main tires. The simmlated values follow the genemal trend of the experimental results, but their aremacy is again compromised be the 
limitations discussed in the first two test cases.

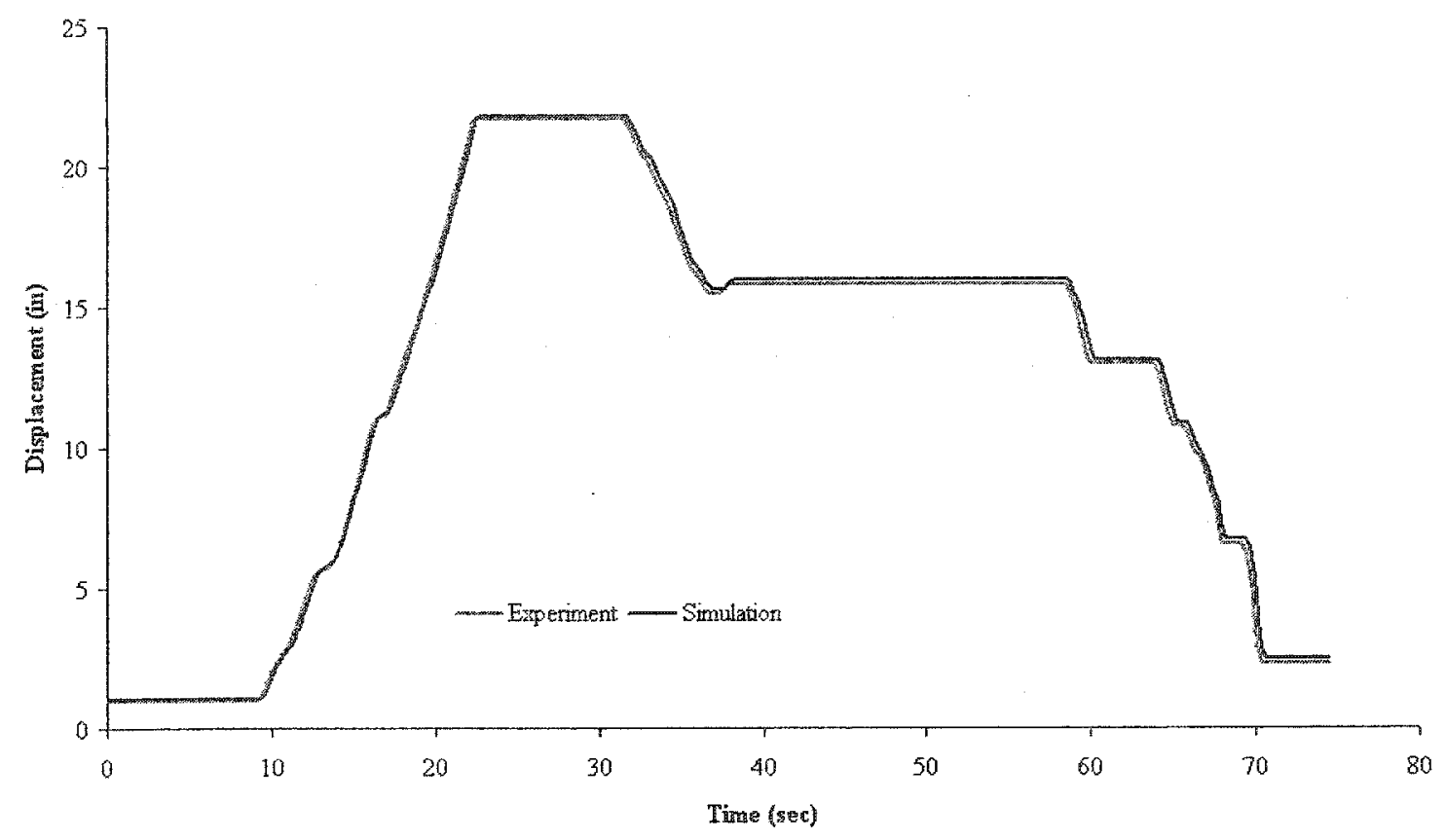

Figure 3.48: Comparison of the RSD claw x-position for Case 5 


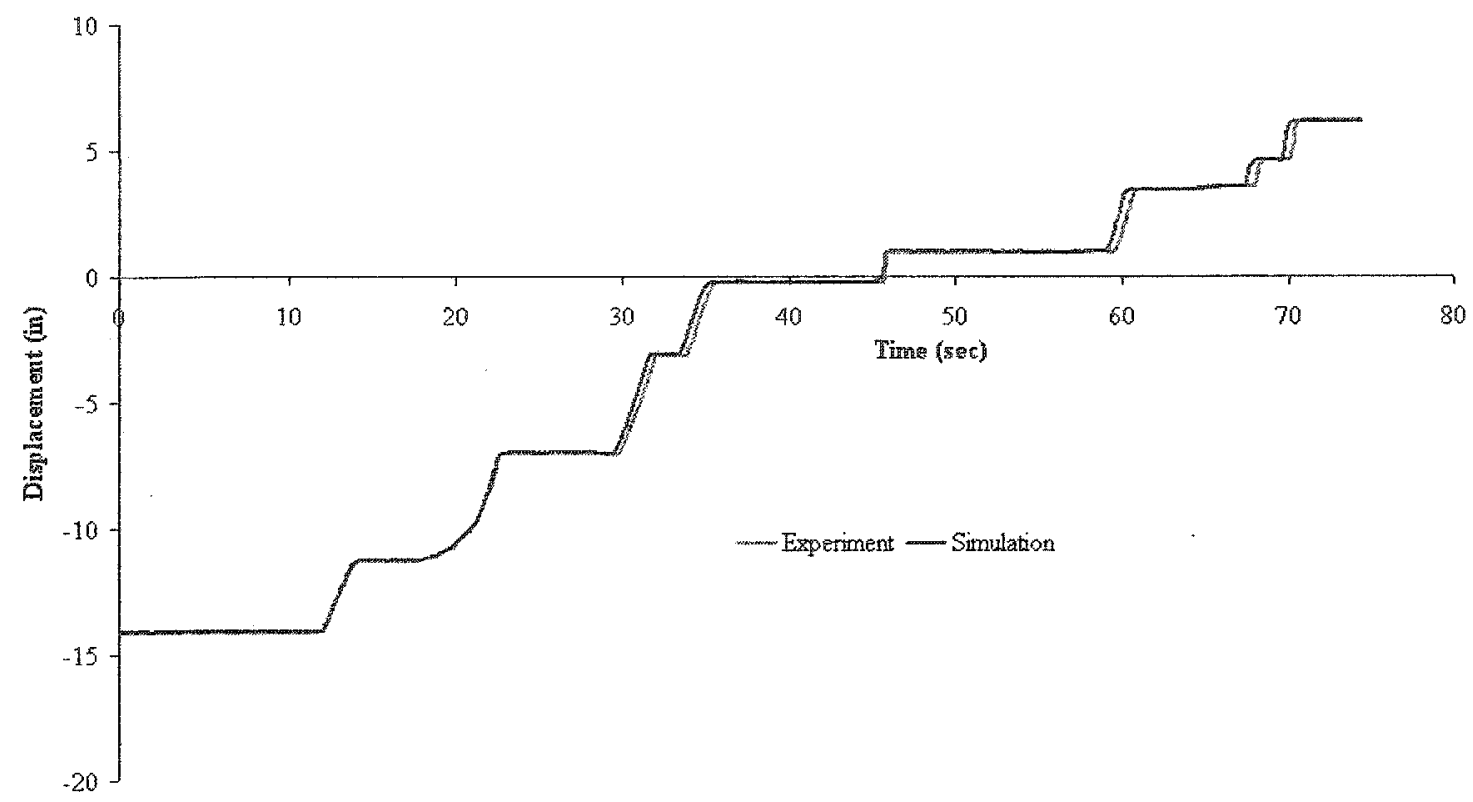

Figure 3.49: Comparison of the RSD claw y-position for Case 5

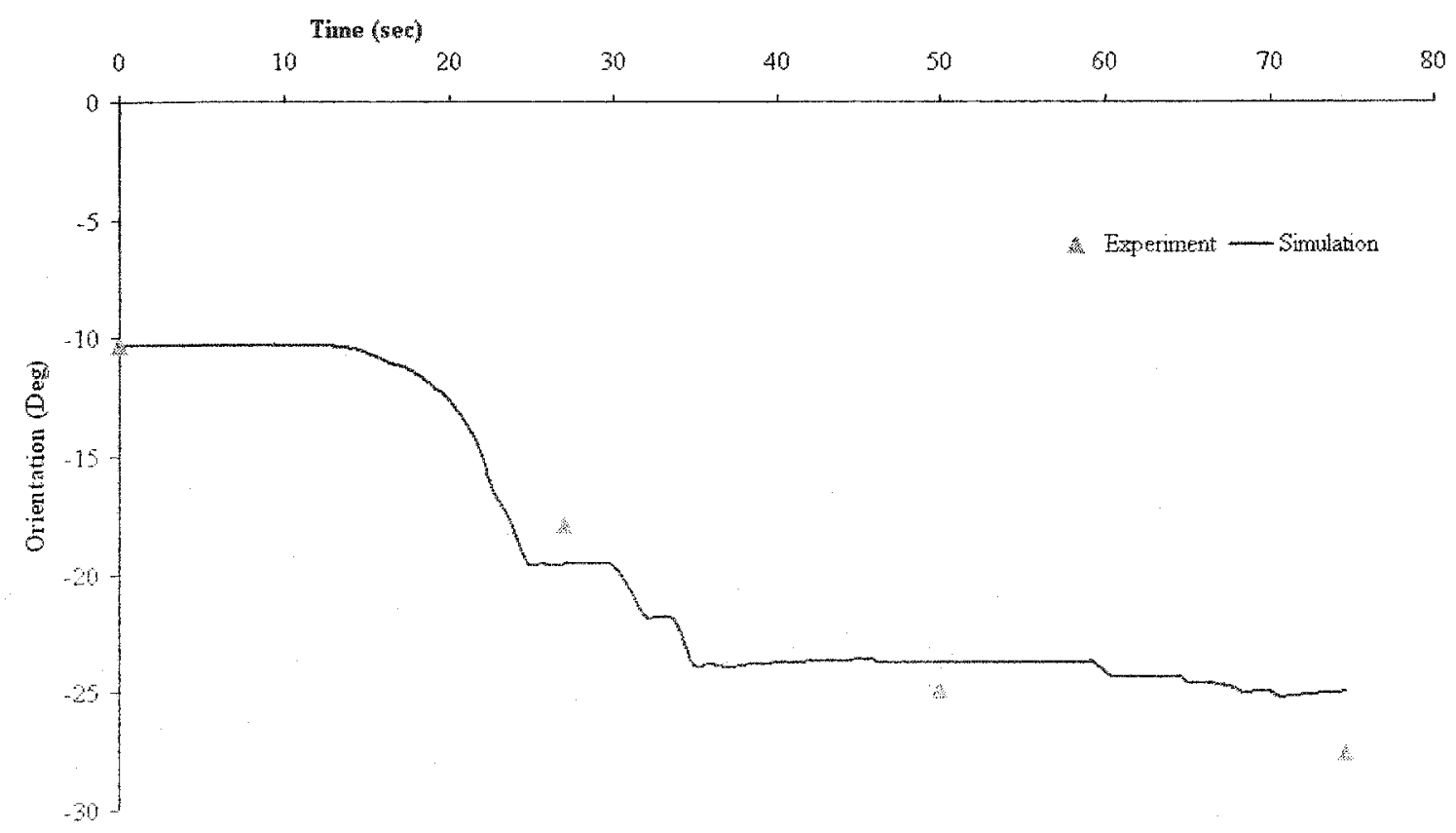

Figure 3.50: Comparison of DLTV orientation for Case 5 


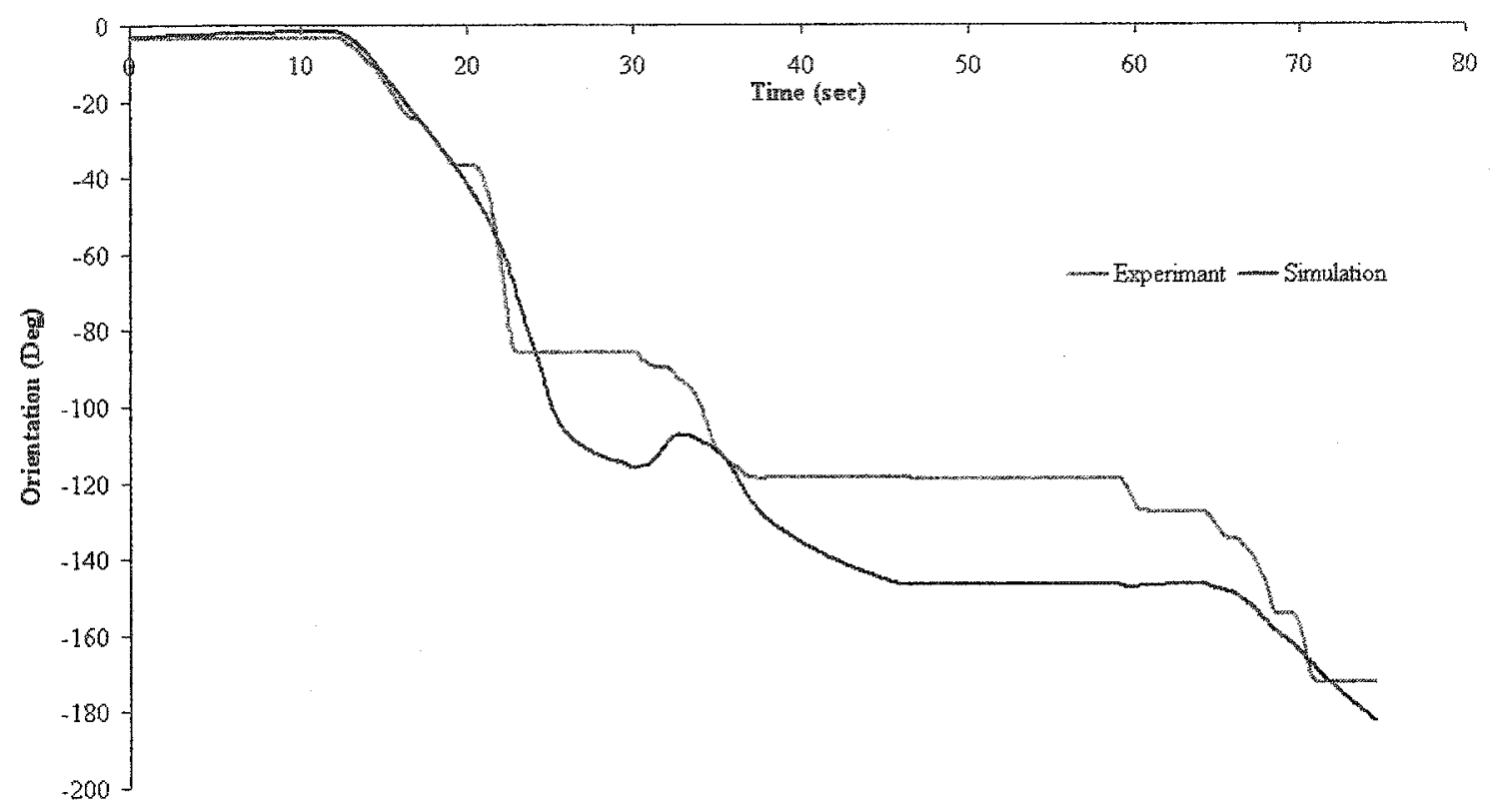

Figure 3.51: Comparison of castor orientation for Case 5

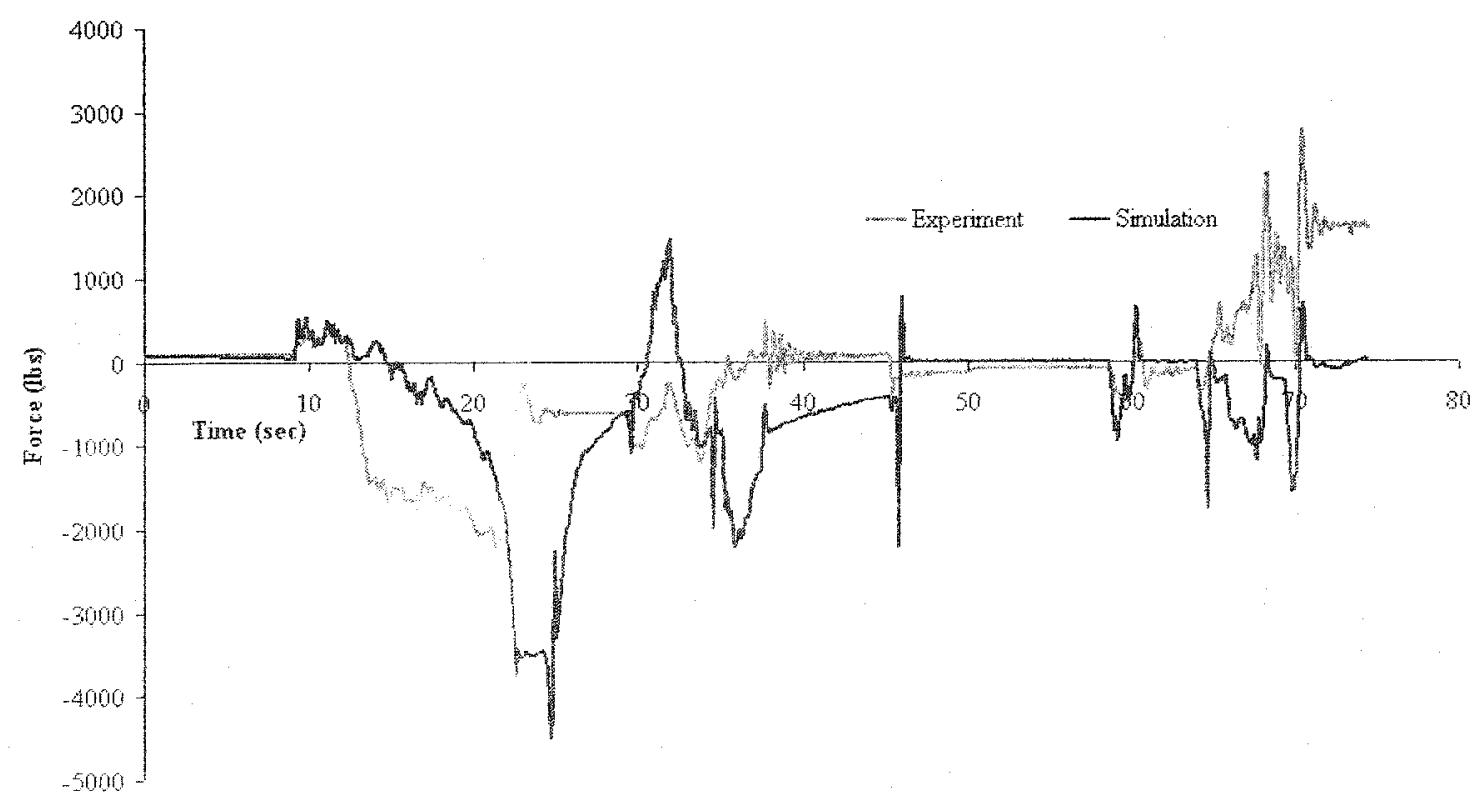

Figure 3.52: Comparison of the fore areting on the man probe in the DLTV's $x$-direction for Case 5 


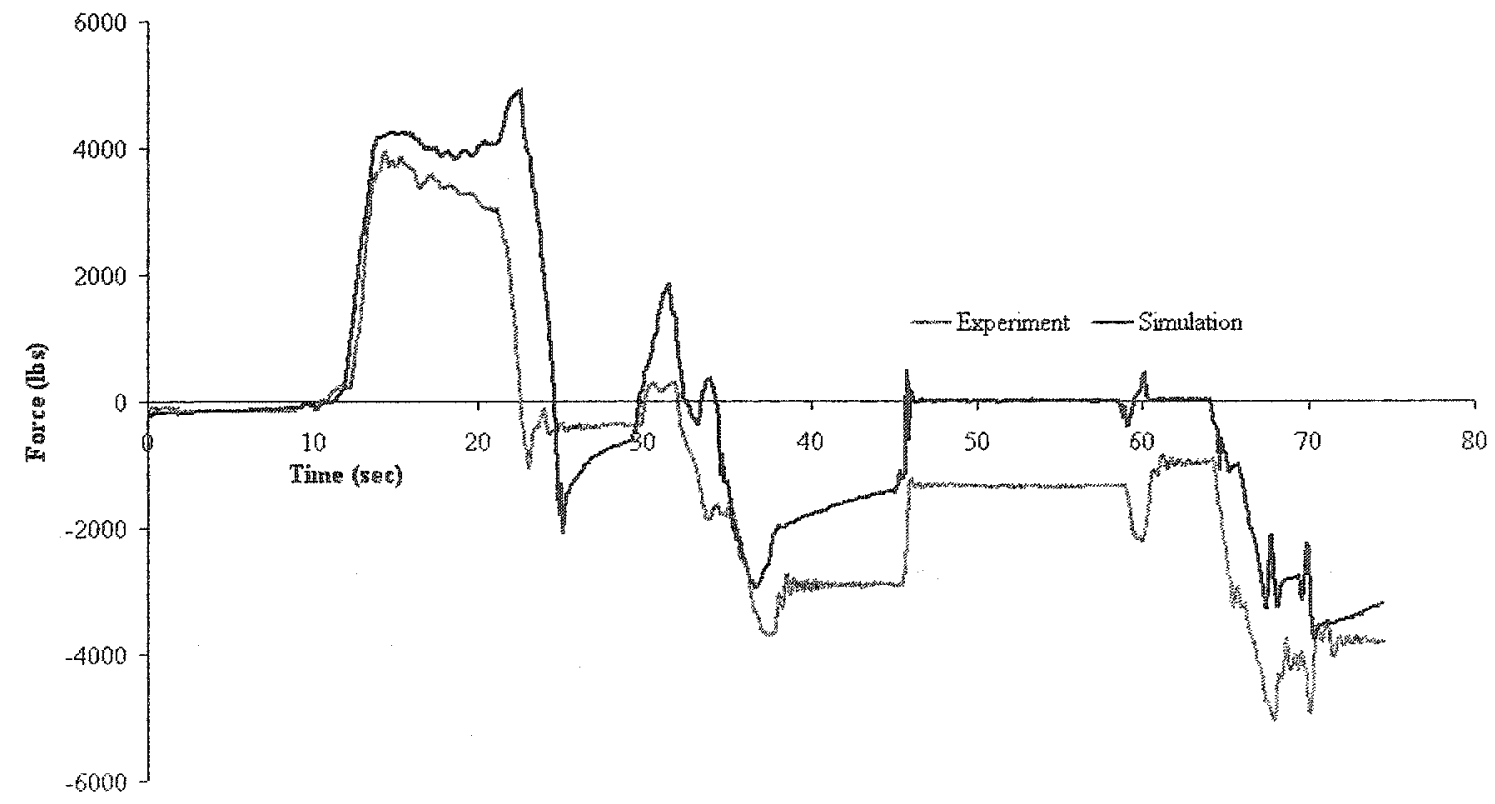

Figure 3.53: Comparison of the force acting on the main probe in the DLTV's $y$-direction for Case 5 


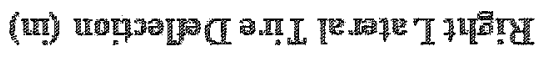

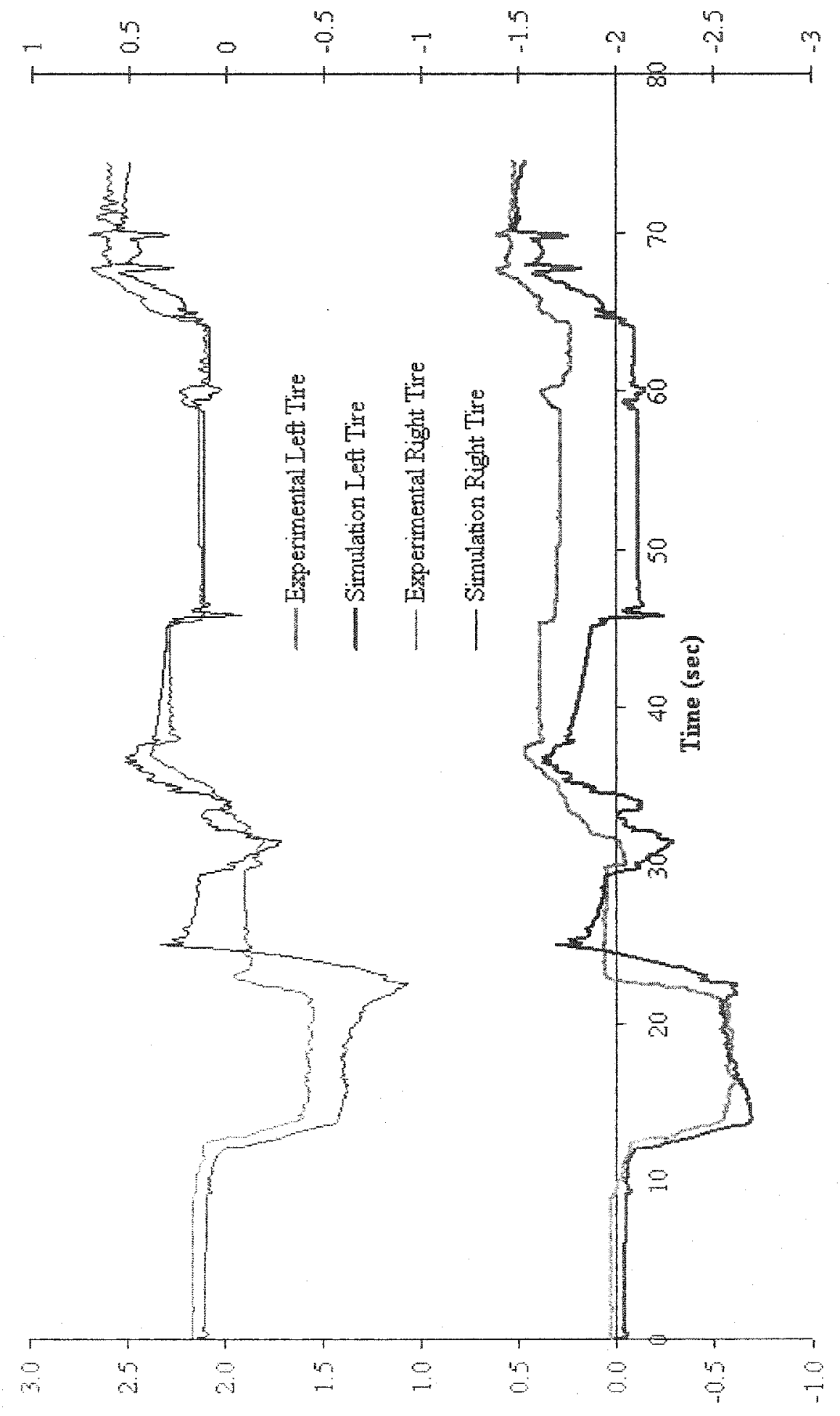

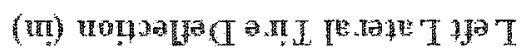

Figure 3.54: Conparison of the hiteral tire deflections for Case 5 


\section{Case 6: Traversing with Lateral Tire Deflections and a Tail Probe}

For this test case the DLTV was set up as shown in Figure 3.55 aligned with the centreline of the ASIST track but with an initial lateral force. The castor assembly was fixed in the aligned position and a tail probe was used.

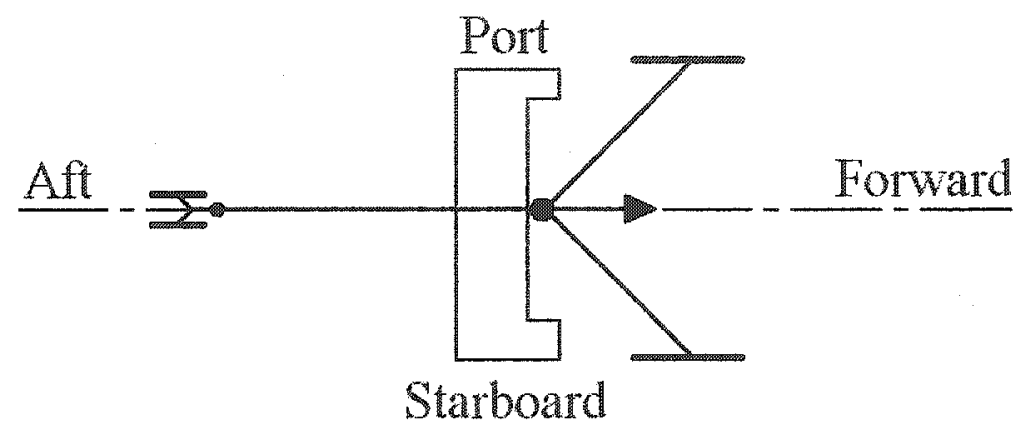

Figure 3.55: Initial position of DLTV and direction RSD is moved for Case 6

The purpose of this case was to test the relaxation of the lateral deflections in the main tires. At this point in time a tail probe option is not available in the HeliMan simulation. The tail probe is a pin that is extended into the traversing track so the tail of the helicopter remains aligned with the track. This effect was introduced into Heliman by giving the castor tires very large lateral tire stiffnesses. This allowed them to roll in the longitudinal direction but have minimal lateral movement.

The DLTV was traversed in the aft direction, while the lateral position of the RSD claw was kept constant. Figures 3.56 and 3.57 show the plotted RSD claw positions. The values used in the simulation match those recorded during the experiment. The lateral RSD claw position does drift a quarter of an inch over the duration of the experiment. This occurred increnentally when the system was shut down to do the hand meanrements as previously discrused.

Figures 3.58 and 3.59 show the plotted simulation results for the forces acting on the nain probe. The predicted $x$ component of the main probe foree was fomd to be moneous 
for the same reasons discussed in the first two cases. A conservative spring-like force is acting in the longitudinal direction of the DLTV. The simulation only applies the nonconservative rolling resistance force. The simulated y component of the main probe force does not decay as quickly as the experimental values. This was likely caused by the drifting of the lateral RSD claw position. HeliMan assumes the lateral RSD claw position remains constant. Each time the system was shut down the force applied to the probe decays with the lateral RSD claw position. The stopping points are identified by the diamonds in Figure 3.59.

Figure 3.60 shows the simulated lateral tire deflections compared to those recorded during testing. Again the load sharing trend of the simulated deflections can be observed with the left and right lateral tire deflections converging. The experimentally measured forces do not do this. The simulated lateral deflections do not relax as quickly as the experimental ones due to the lateral force on the main probe staying higher, as discussed above. 


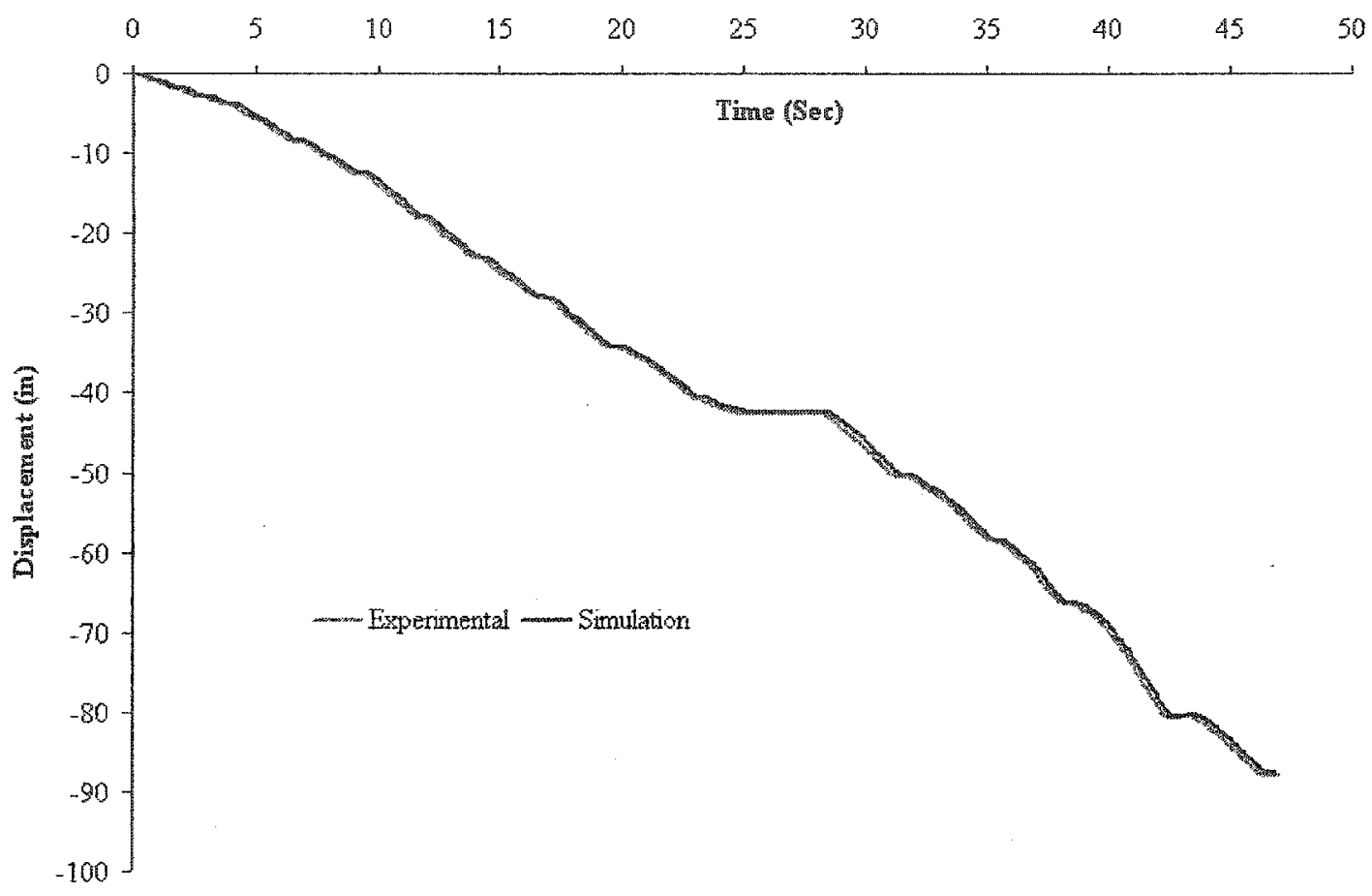

Figure 3.56: Comparison of the RSD claw x-position for Case 6

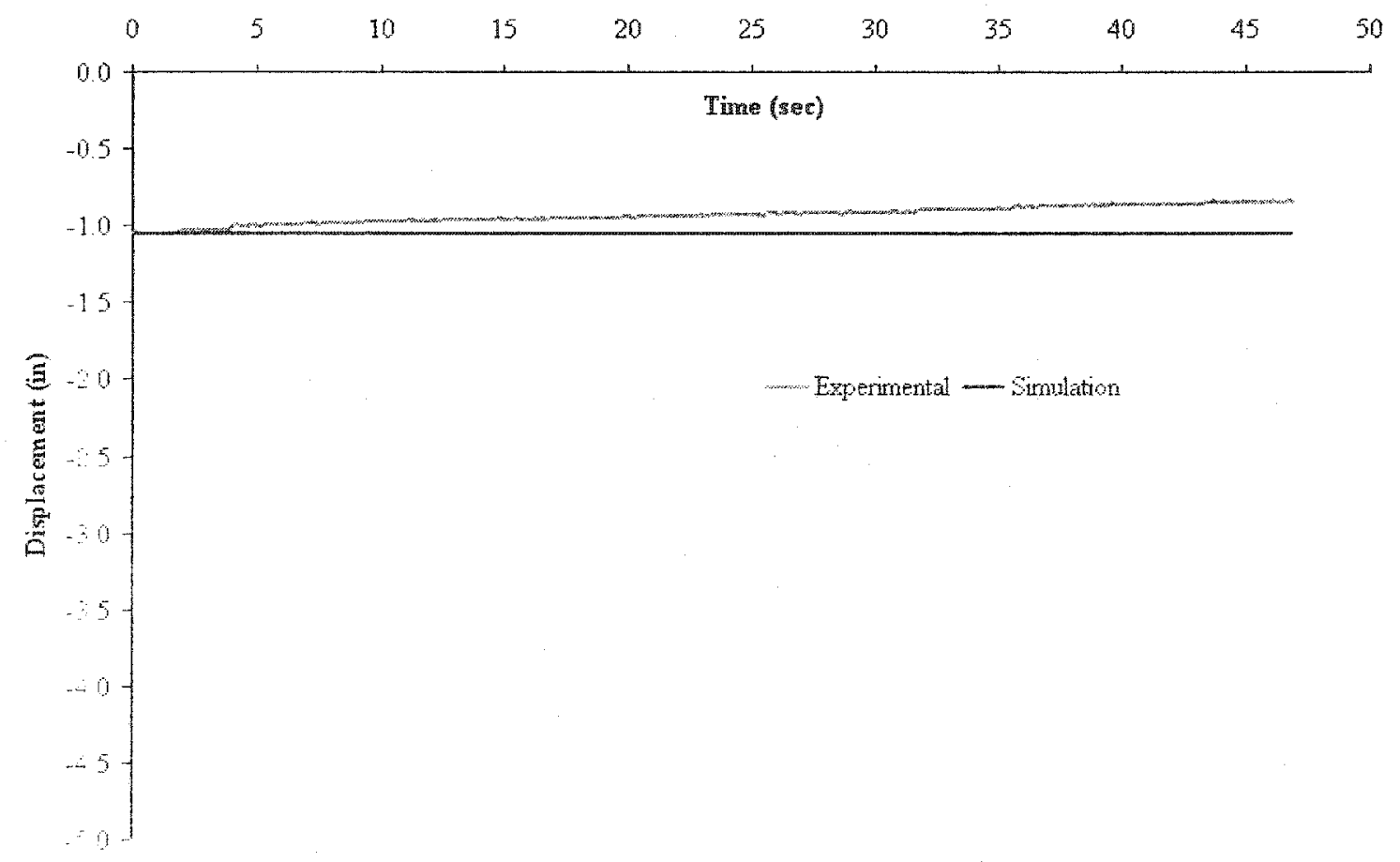

Figure 3.57: Comparison of the RSD claw y-position for Case 6 


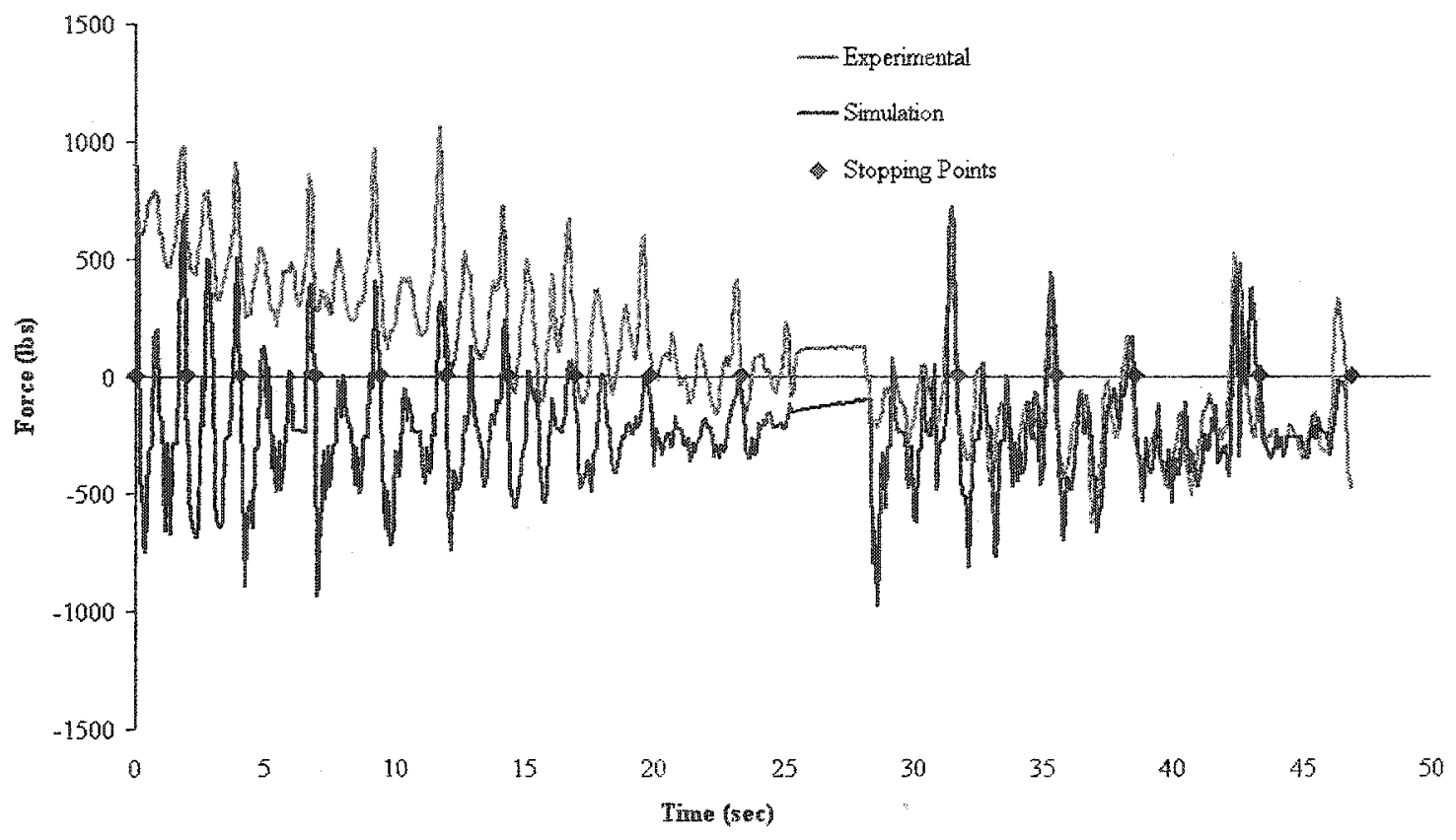

Figure 3.58: Comparison of the force acting on the main probe in the DLTV's $\mathrm{x}$-direction for Case 6

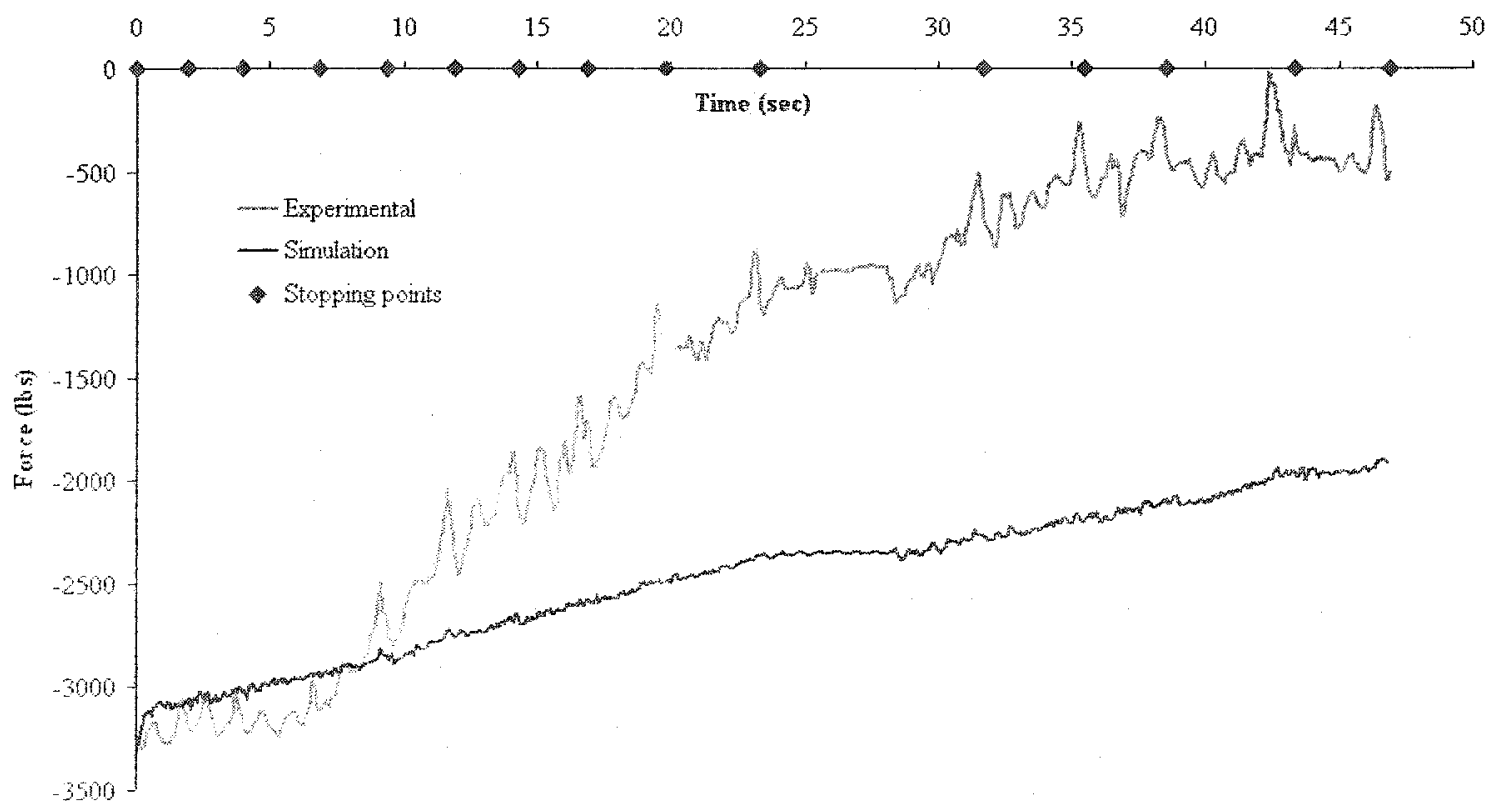

Figure 3.59: Comparison of the fince acting on the main probe in the DITV's v-ripe tion for Case 6 


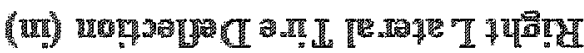

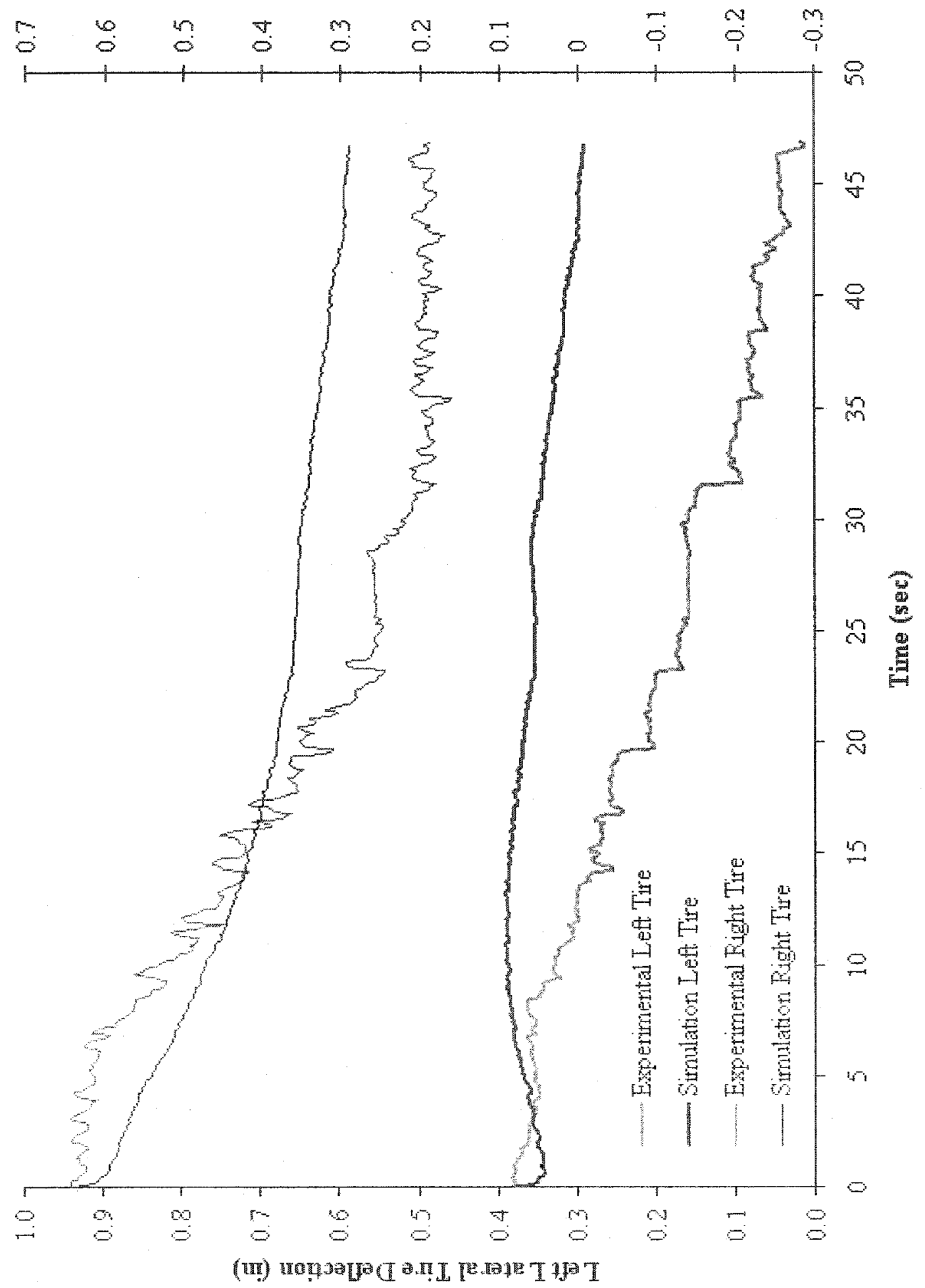

Figure 3.60: Comparison of the lateral tire deftections for $\mathrm{Ca} \cdot 6$ 


\section{Chapter 4}

\section{Discussion and Conclusion}

This project has achieved the intended objectives of:

1. Developing a mathematical model which included: the helicopter equations of motion; the tires; the RSD/probe interface and; the optional auxiliary landing gear.

2. Implementing the mathematical model in a realtime interactive computer simulation called HeliMan.

3. Analyzing the performance of the simulation against full-scale experimental data.

Of particular interest was the success of the dynamic formulation for the helicopter dynamics, which included the castor assembly body. To be able to determine and then use the reaction forces at the castor pivot joint to predict the Coulomb friction acting at the joint was mique. This dynamic formulation perfomed well throughout the validation process.

The tire model was fonnd to be the most complex aspect implenented in the simulation. An airplane taxiing tire mode] developed by NASA [34] was fond to bo the closest match for the on-deck manoenving of a helicopter. Helicopters and airplane's use the same tire types for their landing gear. The vertical and lateral stiffness models proposed in this 
report were validated against data produced by the manufacturer for the tires on the DLTV. This tire validation only encompassed a portion of the entire tire model. During the validation stage, concerns where raised about how well the tire model works for this application due to apparent increases in rolling resistance from the large lateral loading. The relatively high lateral loading was found to be somewhat unique to this application of the tire model.

Although limitations where found in HeliMan during the validation process described in Chapter 3, it predicted the orientation of the DLTV and castor wheels reasonably well. The most significant limitation was found to originate in the implemented tire model. During certain manoeuvre types it was observed from the experimental results that the tires experience a conservative longitudinal force, whereas the tire model as applied, only develops a non-conservative rolling resistance force. Another non-conservative force was added to the tire model based on the lateral force acting on the tire (axle friction), but this only improved the simulation results for the first test case.

The sensitivity of the position of the strain gauges on the DLTV probe was analyzed to determine if experimental error could contribute significantly to differences between the measured and simulated longitudinal probe forces. It was found that an obvious placement error would have had to have been made in the strain gauge application to account for the magnitude of the discrepancies observed between experimental results and the simulated results.

It was hypothesized that the discrepancy that is occuring is caused by longitudinal deflections in the tires that are a function of the lateral load. The tire model used onl considers longitudinal tire deflections when a brake is being applied. This is where the helicopter handling application differs from the airplane taxing application. The airplam. only experiences large lateral loads when it is turning, because the propulsive forces are inline with the tires. Buring helicopter handling a direct lateral load can be applied. which 
may be causing this effect.

The most comprehensive way to determine if this hypothesis is correct is to perform tests on the tires specifically and then develop a mathematical model for the behaviour of the tire for the conditions experienced during helicopter on-deck manoeuvring. This work would be an ideal application for the tire test facility at Carleton University.

HeliMan also demonstrated a tendency to balance out the lateral tire deflections in the main tires. If one of the main tires has a significantly larger deflection than the other, the tendency of the helicopter body is to balance the deflection between both of the main tires. This tendency was not observed in the experimental results.

It has been hypothesized that this was caused by the lack of initial lateral deflections on the castor tires. These were not known because the instrumentation was not available to record them while the tests where being conducted. To verify this hypothesis, some tests could be repeated with the castor tires instrumented to measure lateral deflection or at the very least by measuring by hand the initial lateral deflection of the castor tires.

Other general improvements to the experimental procedure besides instrumenting the castor wheels for lateral deflection, could be made to improve their validity. Each test case should be conducted at least three times to rule out any unpredictable anomalies. If a continuous method for recording the orientation of the DLTV is not available, it would be useful to rum each case contimously to reduce the high-frequency content in the measured data. This would also improve the simulation performance by reducing the stopping and starting in the control input.

Once Heliman has been shown to be fully validated it will then be tested in the presence of ship motion. This will be done first by conducting experiments on a motion platform constructed for the second phase of this project [53]. Hriman will then be integrated into Dynaface $\left.^{(}\right)$and further validated in the presence of simulated ship motion. 
Although HeliMan was qualitatively tested by an experienced ASIST operator, some suggestions were made that could not be addressed within the current project scope. Most of these suggestions revolved around the performance of the simulation on the screen, which will be corrected as improvements are made to the mathematical model. Other options that should be made available for the ASIST operator to take advantage are: a centre lock option which locks the probe in the lateral direction when it is over the track; a curved track, which many ships have, along with the ITF; and a tail probe or nose tray to keep the helicopter straight while it is traversing. The scope of these changes are relatively minor and could be accomplished fairly easily. An ideal possibility is to construct a mockup of the ASIST control panel to be interfaced with the simulation to improve the interactive training experience.

Some more significant options have also been suggested. These options are to allow HeliMan to run using ITI's other handling systems, RAST and TC-ASIST. These operate somewhat differently than the ASIST system. RAST is unable to apply a lateral force to the probe to straighten the aircraft, but uses a hydraulic winch system attached to the helicopter's tail to straighten it. TC-ASIST manipulates the aircraft by the landing gear axles and requires a control algorithm to prevent the operator from damaging the aircraft. These options will require significant changes to the RSD/probe model and the dynamic formulation.

HeliMan has provided a large step towards providing a comprehensive on-deck helicopter manoeuving simulation. Hcliman currently provides the capability to simulate the basic behaviour of the ASIST/helicopter system. Heliman is currently applicable for the roles of qualitative demonstration of ASIST performance, operator training. and developing autonomous control algorithms. The proposed refinement of the tire model will be recuired for obt.ining accurate quantitative results for general helicopter manoenving. 


\section{References}

[1] W. E. Hunt. 'Heelicopter', Pioneering with Igor Sikorsky. Airlife Publishing Ltd., 101 Longden Road, Shrewsbury, England, 1998.

[2] J. Atwood. Helicopter haul down and rapid securing device development. Letter to G. Gray at Indal Technologies Inc., 1992.

[3] R. L. Woods and K. L. Lawrence. Modeling and Simulation of Dynamic Systems. Prentice Hall, Upper Saddle River, New Jersey, U.S.A., 1997.

[4] R. G. Langlois, G. Sopher, and A. R. Tadros. Aircraft/Ship Dynamic Interface Simulation: Dynaface, Release 5.0. Indal Technologies Inc., Mississauga, Ontario, Canada, 1999.

[5] R. G. Langlois and A. R. Tadros. State-of-the-art on-deck dynamic interface analysis. In American Helicopter Society 55 th Annual Forum. Montreal. Quebec, Canada, May 1999.

[6] B. L. Apunso and W. F. Jewell. Rotorcraft control strategy in a three-dimensional wind shrar enviromment. In American Heliopter Society 58th Armual Forum. Montreal, Qubec. Canada, June 2002. 
[7] B. Ferrier, T. Applebee, A. Manning, and D. James. Landing period designator visual helicopter recovery aide; theory and real-time application. In American Helicopter Society 56th Annual Forum, Virginia Beach, Virginia, U.S.A., May 2000.

[8] B. Ferrier, J. Duncan, and T. Manning. Minimum air wake and quiescent motion indicator simulator test program. In American Helicopter Society 59th Annual Forum, Phoenix, Arizona, U.S.A., May 2003.

[9] S. J. R. P. Carignan, A. W. Gubbels, and K. Ellis. Assessment of handling qualities for the shipborne recovery task - ads 33 (maritime). In American Helicopter Society 56th Annual Forum, Virginia Beach, Virginia, U.S.A., May 2000.

[10] R. G. Lee and S. J. Zan. Unsteady aerodynamic loads on a helicopter fusalage in a ship airwake. In American Helicopter Society 58th Annual Forum, Montreal, Quebec, Canada, June 2002.

[11] S. J. Zan. Experimental determination of rotor thrust in a ship airwake. Journal of the American Helicopter Society, 47(2):100-108, April 2002.

[12] Jr. R. McKillip, A. Boschitsch, T. Quackenbush, J. Keller, and D. Wachspress. Dynamic interface simulation using a coupled vortex-based ship airwake and rotor wake model. In American Helicopter Socicty 58th Annual Forum, Montreal, Quebec. Canada. June 2002.

[13] D. Lee, N. Sezer-Uzol, J. F. Horn. and L. N. Long. Simulation of helicopter shiphoard launch and recovery with ime-accurate airwakes. In American Helicopter Society $59 t h$ Annual Forum, Phoenix, Arizona, U.S.A., May 2003.

[11] J. A. Keller and E. C. Smith. Analysis and control of the transiont shiphond ingagement beharion of rotor sustems. In Amerian Helicopter Society 55 th Annual Fomm. Montreal, Quebec. Canada. May 1999. 
[15] J. L. Colwell. Effects of flight deck motion in high seas on the hovering maritime helicopter. In American Helicopter Society 58 th Annual Forum, Montreal, Quebec, Canada, June 2002.

[16] R. Bradley and G. Turner. Simulation of the human pilot applied at the helicopter/ship dynamic interface. In American Helicopter Society 55th Annual Forum, Montreal, Quebec, Canada, May 1999.

[17] J. F. O'Hanlan and M. E. McCauley. Motion sickness incidence as a function of the frequency and acceleration of vertical sinusoidal motion. Aerospace Medicine, 1974.

[18] R. W. Schoenberger. Subjective response to very low-frequency vibration. Aviation, Space, and Environmental Medicine, 1975.

[19] R. Graham, A. E. Baitis, and W. G. Meyers. On the development of seakeeping criteria. Naval Engineer's Journal, 104(3), 1992.

[20] J. Wedge and R. G. Langlois. Simulating the effects of ship motion on postural stability using articulated dynamic models. In Proceedings of the SCSC 2003 Summer Computer Simulation Conference, Montreal, Quebec, Canada, July 2003. The Society for Modeling and Simulation International.

[21] A. R. J. M. Lloyd. SEAKEEPING: Ship Behaviour in Rough Weather. Published by A. R. I. M. Lloyd, 26 Spithead Avenue, Gosport, Hampshire, Unitied Kingdom. revised edition odition, 1998.

[22] R. G. Langlois, A. R. Tadros, and M. LaRosa. Investigation of the retationship between helicopter geometrical and inertial parameters ant shipboard serumg requirements. In American Helicopter Sochety 58th Anmual Forum. Montreal. Quebec. Canada, June 2002 . 
[23] A. R. Tadros, R. G. Langlois, and M. LaRosa. Investigation of ship flight deck motion limits for safe embarked helicopter operation. In IPLC, Arlington, Virginia, U.S.A., October 2000. American Helicopter Society, Inc.

[24] E. R. Joelson. Shipborne helicopter - landing, security, manoeuvring, and traversing: Options and implications. In WARSHIP '97: International Symposium on Air Power at Sea, London, U.K., June 1997. The Royal Institution of Naval Architects.

[25] A. R. Tadros, G. Sopher, and R. G. Langlois. A new approach for passive securing at landing and powered handling of helicopters onboard small ships. In WARSHIP 'g8: International Symposium on Surface Warships: The next Generation, London, U.K., June 1998. The Royal Institution of Naval Architects.

[26] Indal Technologies Inc. Product description: Aircraft ship integrated secure and traverse (ASIST) system. Marketing Brochure, Mississauga, Ontario, Canada.

[27] F. Wei, E. Baitis, and W. Meyers. Analytical modeling of SH-2F helicopter shipboard operation. Journal of Aircraft, 29(5):877-885, September/October 1992.

[28] A. de Smit. Chss-2/dde winch down securing loads. Technical Report H-1026, Unitied Aircraft of Canada Limited, September 1966.

[29] J. Blackwell, A. M. Arney; N. E. Gilbert, and T. T. Truong. Modelling the helicopter/ship dynamic interface for the seahawk/FFG-7. In Proceedings of the 5th Australian Aeronautical Confcrence. Part 2 (of 2), volume 2, pages 277 284. Melbourne, Australia, September 1993. The Institution of Engineers, Australia; The Royal Aeronautical Society, Australian Division, IE Aust.

[30] L. Temoy and R. G. Langlois. User's Guide for the SHIPSIM 4.2 Ship Motion Simulation Program. Indal Terhologies Imnc.. Mississanga. Ontario. Canada. 1999. 
[31] H. Kang, C. He, and H. Saberi. Dynamic interface simulation of rotorcraft shipboard on-deck operation. In American Helicopter Society 59th Annual Forum, Phoenix, Arizona, U.S.A., May 2003.

[32] C. He, H. Kang, D. Carico, and K. Long. Development of a modeling and simulation tool for rotorcraft/ship dynamic interface testing. In American Helicopter Society 58th Annual Forum, Montreal, Quebec, Canada, June 2002.

[33] H. Xin and C. He. A combined technique for inverse simulation applied to rotorcraft shipboard operations. In American Helicopter Society 58th Annual Forum, Montreal, Quebec, Canada, June 2002.

[34] R. F. Smiley and W. B. Horne. Mechanical properties of pneumatic tires with special reference to modern aircraft tires. Technical Report TR R-64, Langley Research Center, NASA, Langley Field, Virginia, U.S.A., 1960.

[35] G. H. Nybakken, R. N. Dodge, and S. K. Clark. A study of the dynamic tire properties over a range of tire constructions. Technical report, The University of Michigan, Ann Arbor, Michigan, U.S.A., March 1973. Prepared for Langley Research Center, NASA as report CR-2219.

[36] G. H. Nybakken and S. K. Clark. Vertical and lateral stiffness characteristies of aircraft tires. Technical report, The University of Michigan. Ann Arbor, Michigan. U.S.A., December 1969. Prepared for Langley Research Center. NASA as report CR-1488.

[37] R. K. Sleeper and E. G. Smith. Planar equations of rollout motion for an aircraft with free or stecrable landing gears. Technical report. Langley Research Center, NASA. Hampton. Virginia. U.S.A., March 1982. 
[38] H. B. Pacejka and I. J. M. Besselink. Magic formula tyre model with transient properties. In Proceedings of the 1997 2nd International Colloquium on Tyre Models for Vehicle Dynamic Analysis, volume 21 of Vehicle System Dynamics, pages 234-249, Berlin, Germany, Febuary 1997. Swets Publishing Services.

[39] M. T. P. van Slagmaat. Tire models in aircraft landing gear simulation. In Proceedings of the 1st International Colloquium on Tyre Models for Vehicle Dynamics Analysis, volume 21 of Vehicle System Dynamics, pages 108-115, Delft, Netherlands, October 1991. Swets Publishing Services.

[40] J. Zhou, J. Y. Wong, and R. S. Sharp. Multi-spoke, three plane tyre model for simulation of transient behaviour. Vehicle System Dynamics, 31(1):35-45, January 1999.

[41] A. Ghazi Zadeh and A. Fahim. An analytical transient tire model. Tire Science and Technology, 29(2):108-132, April/June 2001.

[42] M. Hashimoto, H. Yoshinaga, H. Yoshikawa, N. Morikawa, T. Uekusa, and S. Asai. Three-dimensional finite element analysis of loaded tires. NEC Research $\&$ Development, 35(4):477-487. October 1994 .

[43] H. J. Yu and H. Abontorabi. Dynamics of tire, wheel, and suspension assembly. Tire Science and Technolugy. 29(2):66 78, April/June 2001.

[44] M. Shiraishi. H. Yoslinagn. A. Miyori, and E. Takahashi Simulation of dynamically rolling tire. Tire Scince and Technology. 28(4):264 276. October 2000.

[45] C. R. Hogg. Simulation of Ground Handling of Taximg Akreraft. PhD thesis. Department of Mechanical and Mamuacturing Engineering. University of Brighton. Brighton. U.K., June 1993. 
[46] R. K. Sleeper and $\mathbb{E}$. G. Smith. A transformation method for deriving, from a photograph, position and heading of a vehicle in a plane. Technical report, Langley Research Center, NASA, Hampton, Virginia, U.S.A., May 1976.

[47] B. Mallock, C. J. Harris, C. R. Hogg, and D. Jones. Trimming of flight simulation ground models. Proceedings of the Institution of Mechanical Engineers, Part G: Journal of Aerospace Engineering, 211(2):91-102, 1997.

[48] A. Self, C. Hogg, D. Pearce, and G. Kapadoukas. The simulation of taxiing and low flying aircraft. In F. Maceri and G. Iazeolla, editors, EUROSIM '92 Simulation Congress : proceedings of the 1992 EUROSIM Conference, pages 263-268, Capri, italy, September 1993. North-Holland.

[49] G. G. Kapadoukas, A. W. Self, and C. J. Harris. Flight simulation: Eigenvalue analysis during taxiing. In Chinni, editor, Proceedings of the Military, Government and Aerospace Conference.

[50] J. Y. Wong. Theory of Ground Vehicles. John Wiley \& Sons, Inc., 605 Third Avenue, New York, NY, U.S.A., 2nd edition, 1993.

[51] N. S. Currey. Aircraft Landing Gear Design: Principles and Practice. AIAA Education Series. American Institute of Aeronautics and Astronautics Inc. Washington, D.C., U.S.A., 1988.

[52] F. M. White. Fluid Mechanies. Scham's Ontine Series in Mechanical Engineering. Schaum Division, MeGraw-Hill, Inc, Princeton Roal. S-1, Hightstown, New Jersey, U.S.A., 3rd edition. 1994.

[53] A. Feldman. Devepment of an experinestal motion platform for the preliminary inrstigation into the autonomons on-deck helicopter manoenving ung ASIST. Master's thesis, Corleton University, 2004. In preperintion. 
[54] S. C. Chapra and R. P. Canale. Numerical Methods for Engineers. McGraw-Hill Publishing Company, 2nd edition, 1988. 


\section{Appendix A}

\section{HeliMan Users Guide}

The first requirement to run HeliMan is that the computer must have DirectX 8.0 or higher installed on it. This provides the drivers needed for the joystick to work properly. Problems will be experienced without Direct X 8.0, even if the joystick option is not activated.

HeliMan was programmed in FORTRAN 77 to make it easier to integrate into Dynaface ${ }^{\circledR}$ in the future. Two input files, INPUT.DAT and AIRCRAFT.DAT, are used

to control the simulation. AIRCRAFT.DAT is the standard file used by Dynaface ${ }^{\mathbb{B}}$ to input the helicopter properties. INPUT.DAT contains the information HeliMan needs to run in a prescribed manner and any helicopter properties that are not available in the AIRCRAFT.DAT file. The units of the values in both files are made up of slugs, feet. pounds and degrees. As an example the tire pressures are in $1 \mathrm{bs} / \mathrm{ft}^{2}$.

In addition to the interactive joystick interface, HeliMan can be controlled using imput files. The two options available are the JOYSTICKINPUT.INP file and the FORCEINPUT.INP. These files can be generated by hand or by Heliman. The total length of the simulation must be the first value in the file. All of the input files that have been describer must be in the same folder as the HELIMAN.EXE file. Sample versions of JOYSTICKINPUT.INP and FORCEINPUT.INP are presented next. 


\section{Sample JOYSTICKINPUT.INP file}

$\begin{array}{llllll}1 & 46.89 \mathrm{~d} 0 & & & & \\ 2 & 0.00 \mathrm{~d} 0 & 32.00 \mathrm{~d} 0 & 3.00 \mathrm{~d} 0 & 0 & 0 \\ 3 & 0.10 \mathrm{~d} 0 & 31.00 \mathrm{~d} 0 & 3.00 \mathrm{~d} 0 & 0 & 0 \\ 4 & 0.20 \mathrm{~d} 0 & 30.00 \mathrm{~d} 0 & 3.00 \mathrm{~d} 0 & 0 & 0 \\ 5 & 0.30 \mathrm{~d} 0 & 29.00 \mathrm{~d} 0 & 3.00 \mathrm{~d} 0 & 0 & 0 \\ 6 & 0.40 \mathrm{~d} 0 & 28.00 \mathrm{~d} 0 & 3.00 \mathrm{~d} 0 & 0 & 0\end{array}$

The first value in the file on row 1 is the length, in seconds, of the simulation run. The first column is the time in seconds. The second and third columns are the lateral and longitudinal joystick inputs for that given time. The last two columns represent the pressing of the float button and the castor toggle button respectively. A 1 identifies that the button has been pressed.

\section{Sample FORCEINPUT.INP file}

$\begin{array}{llll}1 & 36.09 \mathrm{~d} 0 & & \\ 2 & 0.00 \mathrm{~d} 0 & 147.225449 \mathrm{~d} 0 & -274.706116 \mathrm{~d} 0 \\ 3 & 0.10 \mathrm{~d} 0 & 193.953461 \mathrm{~d} 0 & -462.252228 \mathrm{~d} 0 \\ 4 & 0.20 \mathrm{~d} 0 & 226.065002 \mathrm{~d} 0 & -667.351929 \mathrm{~d} 0 \\ 5 & 0.30 \mathrm{~d} 0 & 250.841629 \mathrm{~d} 0 & -816.192932 \mathrm{~d} 0 \\ 6 & 0.40 \mathrm{~d} 0 & 285.171326 \mathrm{~d} 0 & -938.345337 \mathrm{~d} 0\end{array}$

The first value in the file on row 1 is the length. in seconds, of the simulation run. The first column is the time in seconds. The second and third columns are the global $x$ and $y$ components of the force being applied at the probe. This control type bypasses the RSD model.

A simplo INPUT.DAT file is now presented along with descriptions of the values wedect. The actual INPUT.DAT file has short descriptions of each value. but these have ben removed for clarity. HeliMan uses FORTRAN's double precision variable type for all 
of its calculations. Therefore, the values input should also be in double precision format, ie ${ }^{*}{ }^{*} \mathrm{~d}^{*}$. Integer values are also used as option flags. The line numbers are not included as part of the input files.

\section{Sample INPUT.DAT file}

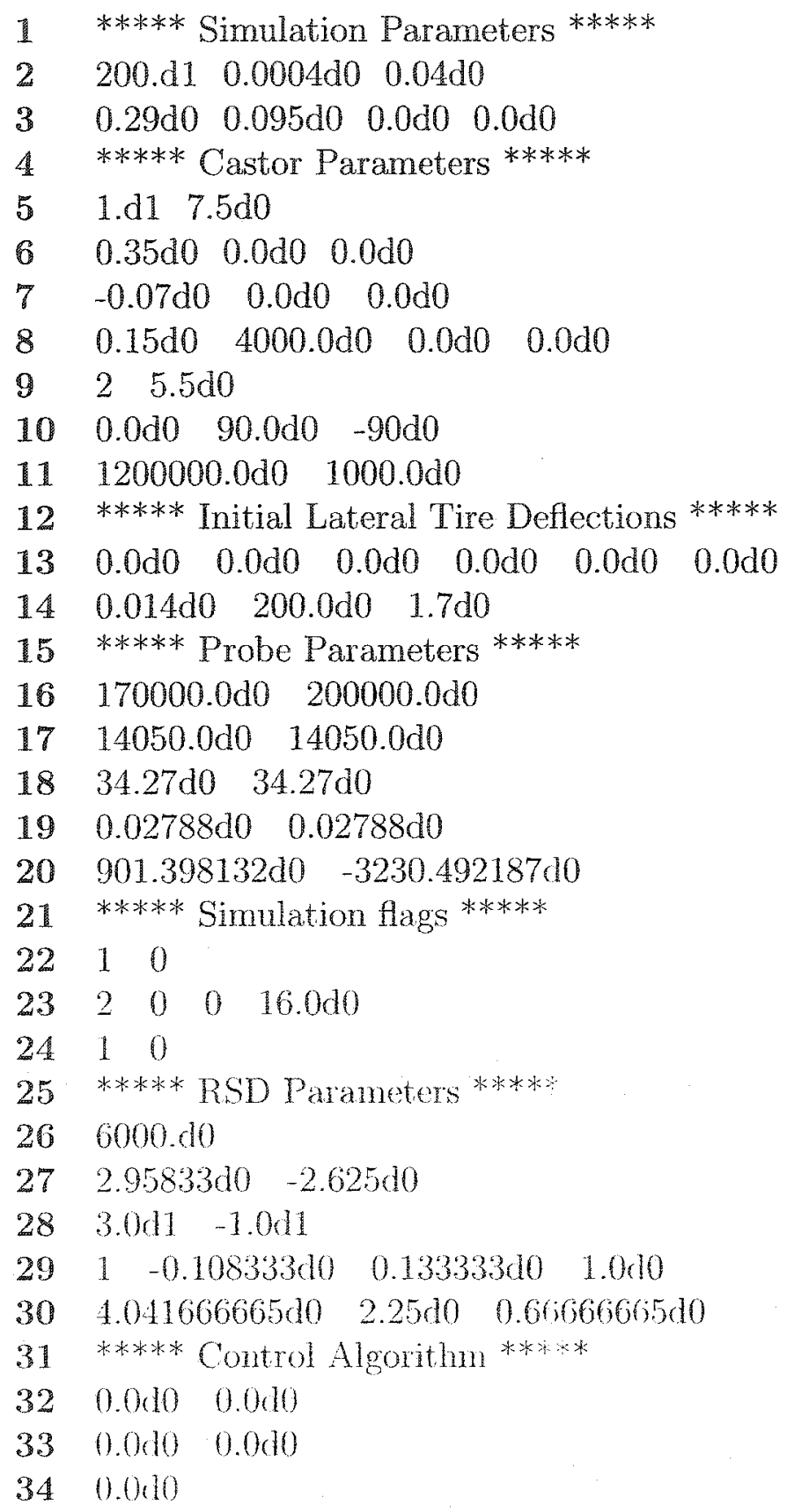




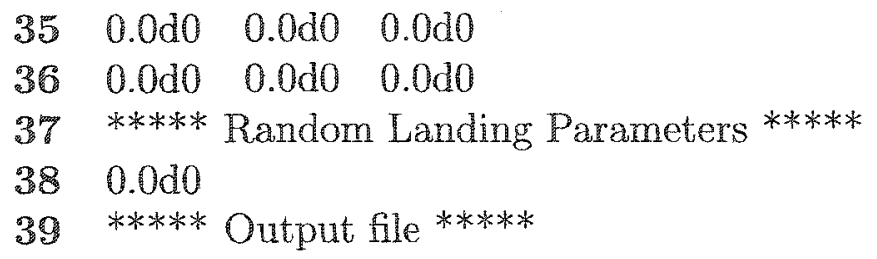

40 Put a 1 beside the variable(s) to be outputted.

Only 15 variables can be outputted at a time.

41 Time

421 Lat deflection of 1st left main tire

430 Lat deflection of 2 nd left main tire

441 Lat deflection of 1 st right main tire

450 Lat deflection of 2 nd right main tire

46 Lat deflection of 1 st castor tire

470 Lat deflection of 2 nd castor tire

$481 \times$ Position of RSD

491 y Position of RSD

$510 \times$ Position left main tire

$520 \mathrm{y}$ Position left main tire

$530 \times$ Position right main tire

540 y Position right main tire

$550 \times$ Position castor pivot

560 y Position castor pivot

$570 \mathrm{x}$ Position mass centre

$580 \times$ Velocity of mass centre

590 y Position mass centre

$600 \mathrm{y}$ Velocity of mass centre

611 Helicopter orientation

621 Castor Orientation

630 Lateral deffection 1st left main tire

64. 0 Global drag force in the $\mathrm{x}$

650 Global drag force in the $y$

$660 \mathrm{x}$ deflection of probe(deltaR $(1))$

$670 \mathrm{y}$ deflection of probe $(\operatorname{deltaR}(2))$

$680 \mathrm{x}$ change in deflection of probetdeta $\mathrm{V}(1)$ )

$690 \mathrm{y}$ change in deflection of probe(deltav(2))

$701 \times$ Probe force in helicopters frame

$711 \mathrm{y}$ Probe force in helicopters frame

$720 \times$ force of 1 st left main tire (global)

$730 \mathrm{y}$ fore of 1 st left main tire (global)

$740 \times$ force of 2 nd left main tire (global)

750 y force of 2 nd left main tire(global)

$760 \times$ force of 1 st right main tire (global)

770 y force of 1st right main tire(global) 
$780 \times$ force of 2 nd right main tire (global)

$790 \mathrm{y}$ force of 2 nd right main tire(global)

$800 \mathrm{x}$ force of 1 st castor tire(global)

$810 \mathrm{y}$ force of 1st castor tire(global)

$820 \times$ force of 2 nd castor tire (global)

$830 \mathrm{y}$ force of 2 nd castor tire(global)

$840 \times$ force at castor joint (global)

$850 \mathrm{y}$ force at castor joint (global)

860 Torque at castor joint

87 Skidding flag

88 ***** Aerodynamic Parameters *****

$890.0 \mathrm{~d} 0 \quad 0.0 \mathrm{~d} 0 \quad 0.00234 \mathrm{~d} 0$

$90 \quad 35.0 \mathrm{~d} 0 \quad 0.0 \mathrm{do} \quad 20.0 \mathrm{~d} 0 \quad 57.08 \mathrm{~d} 0$

$91 \quad 35.0 \mathrm{~d} 0 \quad 0.0 \mathrm{~d} 0 \quad 20.0 \mathrm{~d} 0 \quad 461.5 \mathrm{~d} 0$

$92 * * * * *$ Vertical Tire Load *****

930

$94 \quad 0.0 \mathrm{~d} 0 \quad 0.0 \mathrm{~d} 0 \quad 0.0 \mathrm{~d} 0$

\section{INPUT.DAT value descriptions}

Line 2 The first value is the length of time the simulation is to run. The second value is the integration time step. The third value is the graphical frame rate and the rate of outputting values into OUTPUT.OUT.

Line 3 The initial helicopter positions or state variables, as described in Figure 2.1 from left to right. $q_{1}$ through $q_{4}$.

Line 5 The mass moment of inctia $\left(I_{C}\right)$ and the mass $\left(m_{C}\right)$ of the castor assmbly.

Line 6 The position of the castur assembly's centre of mass with respect to the pivot axis

$$
\left(R_{B / C}^{c}\right)
$$

Line 7 The position of the castor tire with respect to the castor assombly's centre of mass $\left(R_{c t / C_{1}}^{c}\right)$. If lual tires are present this is the point betwen the tires. 
Line 8 The first two values are the castor joint friction and damping coefficients $\left(\mu_{B}\right.$ and $\left.C_{B}\right)$. The next two values are the initial reaction force in global coordinates acting on the castor joint $\left(\vec{F}_{B}^{g}\right)$.

Line 9 The first value is a flag that indicates the initial position of the castor fixing button on the joystick ( $1=$ free, 2,3 , or $4=$ locked at prescribed angles). The second value is the kingpin inclination angle of the castor assembly $(\kappa)$.

Line 10 These values indicate the castor assembly fixing positions $\left(q_{4}\right)$ for the toggling button on the joystick.

Line 11 These are the stiffness and damping values that are used to fix the castor assembly $\left(K_{f i x}\right.$ and $\left.C_{f i x}\right)$.

Line 13 These values are the initial lateral deflections of up to six tires $\left(d_{y}^{t}\right)$. The first two are for the left main tires, the second two the right main tires, and the last two are for the castor tires.

Line 14 The first two values are the coefficient of rolling resistance $\left(\mu_{r r}\right)$ and the smoothing function coefficient $(\beta)$. The last value is the axle friction coefficient $\left(\mu_{a}\right)$.

Line 16 The probe stiffness $\left(K_{P_{x}}\right.$ and $\left.K_{P_{y}}\right)$.

Line 17 The probe damping coefficient $\left(C_{P_{x}}\right.$ and $\left.C_{P_{y}}\right)$.

Line 18 The small probe displacement stiffiess $\left(K_{P_{s_{x}}}\right.$ and $\left.K_{P_{s_{1 /}}}\right)$.

Line 19 The small probe displacoment $\left(D_{P_{s_{x}}}\right.$ and $\left.D_{P_{s_{y}}}\right)$.

Line 20 Initial force acting on the probe in the helicopters frame of reference $\left(F_{x_{x}}^{h}\right.$ and $\left.F_{P_{y}}^{h}\right)$

Line 22 The first value indicates if an ontput file should be ercited $(1=$ on $/ 0=$ off $)$. The second value indicates if the graphics should be enabled $(1=$ on $/ 0=$ off $)$. 
Line 23 The first value is a flag that indicates how the simulation will be controlled (1-joystick, 2-joystick file, 3-force fle, 4-control algorithm). The second value is a flag that indicates if an input file will be created (0-no, 2-joystick file, 3-force file). The third flag indicates that if a joystick input file is being used the lateral velocity is specified directly and not through the joystick model $(1=$ on $/ 0=$ off $)$. The fourth value is a scaling factor to be adjusted based on the resolution of the computer screen being used.

Line 24 The first flag indicates what type of values will be displayed on the graphics screen (0-none, 1-forces, 2-positions). The second value toggles the on or off the display of the lateral tire force vectors $(1=\mathrm{on} / 0=\mathrm{off})$.

Line 26 The maximum force the ASIST RSD can apply.

Line 27 The lateral RSD travel limits (maximum port and maximum starboard).

Line 28 The longitudinal RSD travel limits (maximum forward and maximum aft).

Line 29 The first flag indicates the direction that the RSD faces (1-fore 0-aft). The next three values are the maximum velocities of the RSD in the negative lateral, positive lateral and the longitudinal directions, respectively.

Line 30 The dimensions of the RSD (width, length and depth of ASIST claw slot).

Lines 32-36 Values to be used for the future development of an autonomous control algorithm.

Line 38 Value used to generate random helicopter landing configurations to test the autonomous rontrol algorithin.

Lines 41-87 Up to fifteen values can be chosen to be outputted during the coure of a simulation rum by placing a 1 in the column next to the values description. The 
value's are output into a file called OUTPUT.OUT.

Line 89 The first two values are the wind velocity in the helicopters frame of reference $\left(V_{w_{x}}^{h}\right.$ and $\left.V_{w_{x}}^{h}\right)$. The last value is the density of air.

Line 90 The position of the centre of pressure with respect to the helicopter's $x$-axis $\left(R_{C P x / H_{x}}^{m}\right.$ and $\left.R_{C P x / H_{y}}^{m}\right)$ in the manufacturer's frame of reference. The last value is the equivalent cross-sectional area of the helicopter for the plane perpendicular to the x-plane $\left(A_{e x}\right)$.

Line 91. The position of the centre of pressure with respect to the helicopter's $\mathrm{y}$-axis $\left(R_{C P y / H_{x}}^{m}\right.$ and $R_{C P y / H_{y}}^{m}$ ) in the manufacturer's frame of reference. The last value is the equivalent cross-sectional area of the helicopter for the plane perpendicular to the y-plane $\left(A_{e y}\right)$.

Line 93 A flag that indicates if the wheel station load distribution will be directly applied $(1=\mathrm{on} / 0=\mathrm{off})$.

Line 94 Indicates the optional static loads that could be used. Otherwise HeliMan calculates the load distribution based on the mass. First is for the left main wheel station, second is the right main wheel station and the last is for the castor wheel station.

Next is a sample AIRCRAFT.DAT file along with descriptions of the values needed. The values contained in this guide do not represent an actual helicopter as helicopter data is proprietiry. Lines that contain values that Dyraface ${ }^{\mathbb{R}}$ uses, but Heliman does not use, have been left blank. These blank lines must be left in the file for Heliman to rum.

Sample AIRCRAFT.DAT file

1 *****: Aircraft Paraneters *****

$2 \quad 30.0 \mathrm{~d} 111-0.00 \mathrm{~d} 0 \quad 19.0 \mathrm{~d} 0$ 


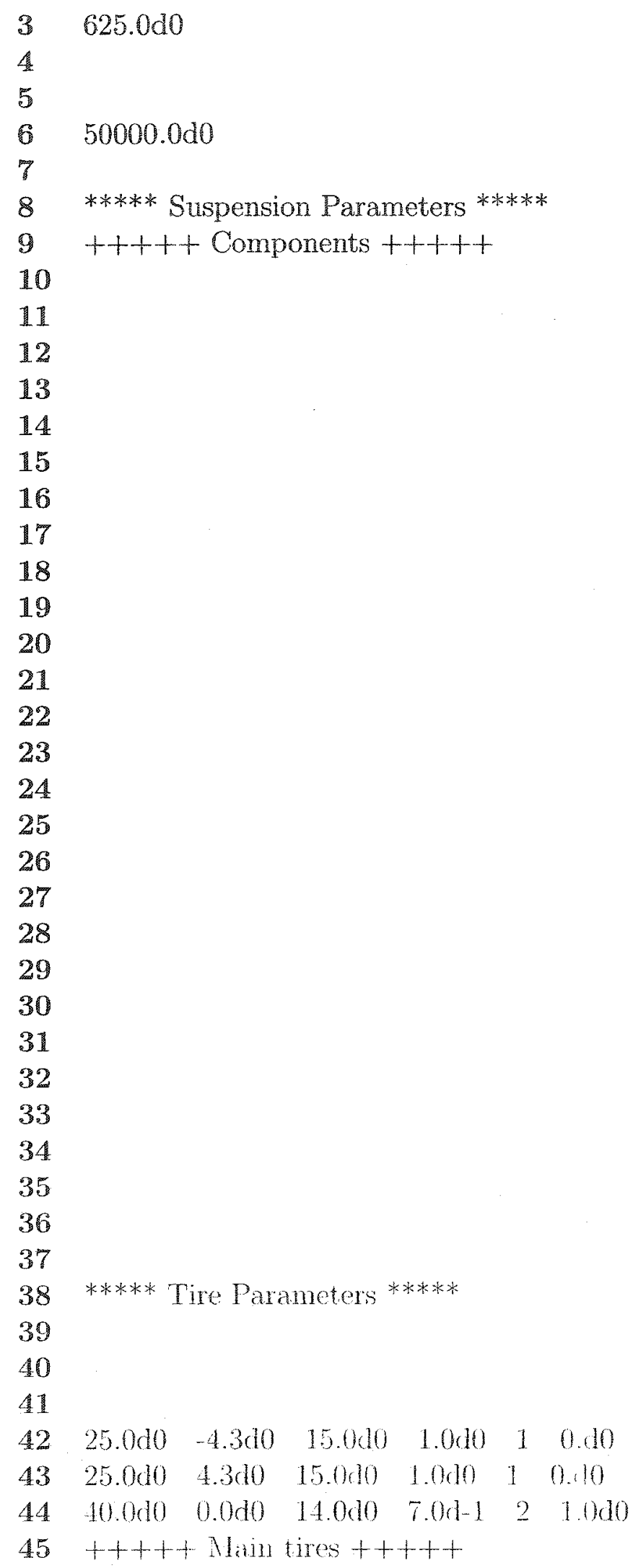




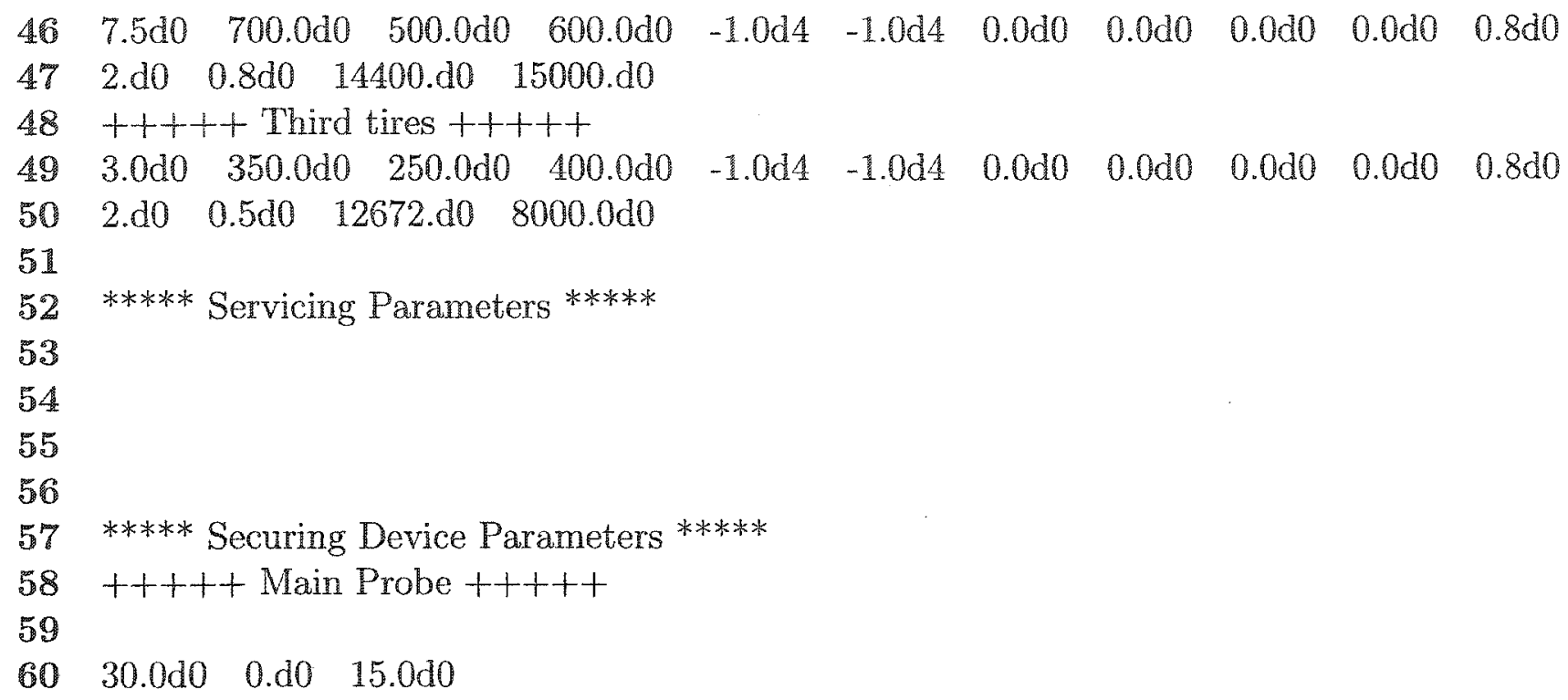

\section{AIRCRAFT.DAT value descriptions}

Line 2 The $x, y, z$ position of the centre of mass in the manufacturer's frame of reference. The Heliman helicopter frame of reference originates from the centre of mass. All of the positions input in the manufacturer's frame of reference are switched to the frame of reference with origin at the helicopter's centre of mass.

Line 3 Mass of the helicopter body $\left(m_{h}\right)$.

Line 6 . Mass moment of inertia of the helicopter body $\left(I_{h}\right)$.

Line 42 The first three values indicate the xyy position of the left main wheel station in the manufacturer's frame of reference. The fourth value is the radins of the left main tire or tires $\left(r_{t}\right)$. The fifth value is a flag that indicates if dual times exist at the left main suspension station $(1=$ no/2=yes $)$. The last value is the spact between the tires.

Line 43 The first three values indicate the $x . y . z$ position of the right main wheel station in the manufacturer's frame of reference. The fouth value is the radin of the right 
main tire or tires $\left(r_{t}\right)$. The fifth value is a flag that indicates if dual tires exist at the right main suspension station $(1=$ no/2=yes $)$. The last value is the space between the tires.

Line 44 The first three values indicate the $x, y, z$ position of the castor wheel station in the manufacturer's frame of reference. The fourth value is the radius of the castor tire or tires $\left(r_{t}\right)$. The fifth value is a flag that indicates if dual tires exist at the castor suspension station $(1=$ no $/ 2=y e s)$. The last value is the space between the tires.

Line 46 The first value is the mass of the main tire. The next three values are the $\mathrm{x}, \mathrm{y}, \mathrm{z}$ damping coefficients of the main tire. The following two are longitudinal and lateral tire stiffnesses if the tire model is being bypassed (negative values indicate the tire model is being used). The next four values are not used by HeliMan, but must be present. The last value is the coefficient of friction between the tire and the flight deck $(\mu)$ for the main tire.

Line 47 The first value is the type coefficient $(\tau)$ for the main tire. The second is the main tire's width $\left(w_{t}\right)$. The third value is the main tire's inflation pressure at zero vertical load (gauge) $\left(P_{o}\right)$. The last value is the rated tire pressure for the main tire $\left(P_{r}\right)$.

Line 49 The first value is the mass of the castor tire. The next three values are the $x, y, z$ danping coefficients of the castor tire. The following two are longitudinal and lateral tire stiffnesses if the tire model is being bypassed (negative values indicate the tire model is being used). The next fon values are not used by HeliMan but must be present. The last value is the coefficient of friction between the tire and the flight deck ( $\mu$ ) for the castor tire.

Line 50 The first value is the trpe coefficient. $(\tau)$ for the castor tire. The second is the 
castor tire's width $\left(w_{t}\right)$. The third value is the castor tire's inflation pressure at zero vertical load (gauge) $\left(P_{0}\right)$. The last value is the rated tire pressure for the castor tire $\left(P_{r}\right)$.

Line 60 Position of the probe in the manufacturer's frame of reference $\left(R_{P_{x}}^{m}, R_{P_{y}}^{m}\right.$ and $\left.R_{P_{z}}^{m}\right)$. 


\section{Appendix B}

\section{Analytical Solution}

An analytical solution for the equations of motion was derived using MATHEMATICA. This solution is complete with exception of the aerodynamic forces that were not included in this derivation but can easily be added to the final solution. The following coefficients are first defined to simplify the resulting equations:

$$
\begin{aligned}
& a_{1}=-r_{B / H_{x}}^{h} \mathrm{~s} q_{3}-r_{B / H_{y}}^{h} \mathrm{c} q_{3}+r_{B / C_{x}}^{c} \mathrm{~s}\left(q_{3}+q_{4}\right)+r_{B / C_{y}}^{c} \mathrm{c}\left(q_{3}+q_{4}\right) \\
& a_{2}=r_{B / C_{x}}^{c} \mathrm{~s}\left(q_{3}+q_{4}\right)+r_{B / C_{y}}^{c} c\left(q_{3}+q_{4}\right) \\
& a_{3}=r_{B / H_{x}}^{h} c q_{3}-r_{B / H_{3}}^{h} \mathrm{~s} q_{3}-r_{B / C_{x}}^{c} \mathrm{c}\left(q_{3}+q_{4}\right)+r_{B / C_{3}}^{c} \mathrm{~s}\left(q_{3}+q_{4}\right) \\
& a_{4}=-r_{B / C_{x}}^{c} c\left(q_{3}+q_{4}\right)+r_{B / C_{y}}^{c} \mathrm{~s}\left(q_{3}+q_{4}\right) \\
& a_{5}=-r_{B / H_{x}}^{h} c q_{3} \dot{q}_{3}^{2}+r_{B / H_{y}}^{h_{h}} \mathrm{~s} q_{3} \dot{q}_{3}^{2}+r_{B / C_{x}}^{c} c\left(q_{3}+q_{4}\right)\left(\dot{q}_{3}+\dot{q}_{4}\right)^{2} \\
& -r_{B / C_{y}}^{c} s\left(q_{3}+q_{4}\right)\left(\dot{q}_{3}+\dot{q}_{4}\right)^{2} \\
& a_{6}=-r_{B / H_{3}}^{h} s_{3} \dot{q}_{3}^{2}-r_{B / H_{3}}^{h} c q_{3} \dot{q}_{3}^{2}+r_{B / C_{x}}^{c} s\left(q_{3}+q_{-1}\right)\left(\dot{q}_{3}+\dot{q}_{4}\right)^{2} \\
& +r_{B / C_{3}}^{c} c\left(q_{3}+q_{4}\right)\left(\dot{q}_{3}+\dot{q}_{4}\right)^{2} \\
& a_{7}=F_{P_{x}}+F_{m i t_{x 1}}+F_{m l t_{x 2}}+F_{m r t_{x 1}}+F_{m r t_{x 2}} \\
& a_{s}=F_{p_{y}}+F_{m l t_{y 1}}+F_{m l t_{y 2}}+F_{m r t_{y j}}+F_{n r t_{y 2}} \\
& a_{9}=\vec{r}_{P / H} \times \vec{F}_{P}+\vec{r}_{m l t / H_{1}} \times \vec{F}_{m l t_{1}}+\vec{r}_{m l t / H_{2}} \times \vec{F}_{m l t_{2}}+\vec{r}_{m+t / H_{1}} \times \vec{F}_{m r t_{1}} \\
& +\vec{r}_{m+H_{2}} \times \vec{F}_{m t_{2}}+M_{B}
\end{aligned}
$$




$$
\begin{aligned}
& a_{10}=F_{c t_{x 1}}+F_{c t_{x 2}} \\
& a_{11}=F_{c t_{y 1}}+F_{c t_{y 2}} \\
& a_{12}=\vec{r}_{c t / C_{1}} \times \vec{F}_{c t_{1}}+\vec{r}_{c t / C_{2}} \times \vec{F}_{c t_{2}}-M_{B}
\end{aligned}
$$

Expressions for the generalized accelerations $\ddot{q}_{1}$ through $\ddot{q}_{4}$ are as follows:

$$
\begin{aligned}
\ddot{q}_{1}= & \frac{1}{m_{h}}\left[a_{7}+\frac{a_{4} a_{12} I_{h} m_{c} m_{h}+I_{c}\left(\left(a_{3}-a_{4}\right) a_{9} m_{c} m_{h}+I_{h}\left(-a_{11} m_{h}+m_{c}\left(a_{8}+a_{6} m_{h}\right)\right)\right)}{m_{c} m_{h}\left(I_{h} r_{B / C_{y}}^{c}\left(c\left(q_{3}+q_{4}\right) r_{B / C_{x}}^{c}-\mathrm{s}\left(q_{3}+q_{4}\right) r_{B / C_{y}}^{c}\right)+I_{c} r_{B / H_{y}}^{h}\left(\mathrm{c} q_{3} r_{B / H_{x}}^{h}-\mathrm{s} q_{3} r_{B / H y}^{h}\right)\right)}\right. \\
& -\left(\left(-a_{4} I_{h} m_{c} m_{h} r_{B / C_{x}}^{c}+I_{c}\left(I_{h}\left(m_{c}+m_{h}\right)+\left(a_{3}-a_{4}\right) m_{c} m_{h} r_{B / H_{x}}^{h}\right)\right)\right. \\
& \times\left(m_{c} m_{h}\left(a_{2} a_{12} I_{h} m_{c} m_{h}+I_{c}\left(\left(a_{1}-a_{2}\right) a_{9} m_{c} m_{h}+I_{h}\left(-a_{10} m_{h}+m_{c}\left(a_{7}+a_{5} m_{h}\right)\right)\right)\right)\right. \\
& \times\left(I_{h} r_{B / C_{y}}^{c}\left(c\left(q_{3}+q_{4}\right) r_{B / C_{x}}^{c}-\mathrm{s}\left(q_{3}+q_{4}\right) r_{B / C_{y}}^{c}\right)+I_{c} r_{B / H_{y}}^{h}\left(c q_{3} r_{B / H_{x}}^{h}-\mathrm{s} q_{3} r_{B / H_{y}}^{h}\right)\right) \\
& -\left(\left(-a_{3}+a_{4}\right) a_{9} I_{c} m_{c} m_{h}+I_{h}\left(-a_{4} a_{12} m_{c} m_{h}+I_{c}\left(a_{11} m_{h}-m_{c}\left(a_{8}+a_{6} m_{h}\right)\right)\right)\right) \\
& \left.\left.\times\left(a_{2} I_{h} m_{c} m_{h} r_{B / C_{y}}^{c}+I_{c}\left(I_{h}\left(m_{c}+m_{h}\right)+\left(-a_{1}+a_{2}\right) m_{c} m_{h} r_{B / H_{y}}^{h}\right)\right)\right)\right) \\
& \left(m_{c} m_{h}\left(I_{h} r_{B / C_{y}}^{c}\left(-c\left(q_{3}+q_{4}\right) r_{B / C_{x}}^{c}-\mathrm{s}\left(q_{3}+q_{4}\right) r_{B / C_{y}}^{c}\right)+I_{c} r_{B / H_{y}}^{h}\left(\mathrm{c} q_{3} r_{B / H_{x}}^{h}-\mathrm{s} q_{3} r_{B / H_{y}}^{h}\right)\right)\right. \\
& \times\left(m _ { c } ^ { 2 } m _ { h } ^ { 2 } ( a _ { 2 } I _ { h } r _ { B / C _ { x } } ^ { c } + ( - a _ { 1 } + a _ { 2 } ) I _ { c } r _ { B / H _ { x } } ^ { h } ) \left(I _ { H } r _ { B / C _ { y } } ^ { c } \left(c\left(q_{3}+q_{4}\right) r_{B / C_{x}}^{c}\right.\right.\right. \\
& \left.\left.-\mathrm{s}\left(q_{3}+q_{4}\right) r_{B / C_{y}}^{c}\right)+I_{c} r_{B / H_{y}}^{h}\left(c\left(q_{3}\right) r_{B / H_{x}}^{h}-\mathrm{s}\left(q_{3}\right) r_{B / H_{y}}^{h}\right)\right)-\left(-a_{4} I_{h} m_{c} m_{h} r_{B / C_{x}}^{c}\right. \\
& +I_{c}\left(I_{h}\left(m_{c}+m_{h}\right)+\left(a_{3}-a_{4}\right) m_{c} m_{h} r_{B / H_{x}}^{h}\right)\left(a_{2} I_{h} m_{c} m_{h} r_{B / C_{x}}^{c}+I_{c}\left(I_{h}\left(m_{c}+m_{c}\right)\right.\right. \\
& \left.\left.\left.\left.+\left(-a_{1}+a_{2}\right) m_{c} m_{h} r_{B / H_{y}}^{h}\right)\right)\right)\right]
\end{aligned}
$$

$$
\begin{aligned}
\ddot{q}_{2}= & \frac{1}{m_{h}}\left[a_{8}+\left(m _ { c } m _ { h } \left(a_{2} a_{12} I_{h} m_{c} m_{h}+I_{c}\left(\left(a_{1}-a_{2}\right) a_{9} m_{c} m_{h}+I_{h}\left(-a_{10} m_{h}\right.\right.\right.\right.\right. \\
& \left.\left.\left.+m_{c}\left(a_{7}+a_{5} m_{h}\right)\right)\right)\right)\left(I_{h} r_{B / C_{y}}^{c}\left(c\left(q_{3}+q_{4}\right) r_{B / C_{x}}^{c}-s\left(q_{3}+q_{4}\right) r_{B / C_{y}}^{c}\right)+I_{c} r_{B / H_{y}}^{h}\right. \\
& \left.\times\left(c\left(q_{3}\right) r_{B / H_{x}}^{h_{1}}-s\left(a_{3}\right) r_{B / H_{y}}^{h_{h}}\right)\right)-\left(\left(-a_{3}+a_{4}\right) a_{9} I_{c} m_{c} m_{h}\right. \\
& \left.+I_{h}\left(-a_{1} h_{12} m_{c} m_{h}+I_{c}\left(a_{11} m_{h}-m_{c}\left(a_{8}+a_{6} m_{h}\right)\right)\right)\right)\left(a_{2} I_{h} m_{c} m_{h} r_{B / C_{y}}^{c}\right. \\
& \left.\left.+I_{c}\left(I_{h}\left(m_{c}+m_{h}\right)+\left(-a_{1}+a_{2}\right) m_{c} m_{h} r_{B / H_{y}}^{h}\right)\right)\right)
\end{aligned}
$$




$$
\begin{aligned}
/ & \left(\left(m _ { c } ^ { 2 } m _ { h } ^ { 2 } ( a _ { 2 } I _ { h } r _ { B / C _ { x } } ^ { c } + ( - a _ { 1 } + a _ { 2 } ) I _ { c } r _ { B / H _ { x } } ^ { h } ) \left(I _ { H } r _ { B / C _ { y } } ^ { c } \left(c\left(q_{3}+q_{4}\right) r_{B / C_{x}}^{c}\right.\right.\right.\right. \\
& \left.\left.-\mathrm{s}\left(q_{3}+q_{4}\right) r_{B / C_{y}}^{c}\right)+I_{c} r_{B / H_{y}}^{h}\left(c\left(q_{3}\right) r_{B / H_{x}}^{h}-s\left(q_{3}\right) r_{B / H_{y}}^{h}\right)\right)-\left(-a_{4} I_{h} m_{c} m_{h} r_{B / C_{x}}^{c}\right. \\
& \left.+I_{c}\left(I_{h}\left(m_{c}+m_{h}\right)+\left(a_{3}-a_{4}\right) m_{c} m_{h} r_{B / H_{x}}^{h}\right)\right)\left(a_{2} I_{h} m_{c} m_{h} r_{B / C_{x}}^{c}+I_{c}\left(I_{h}\left(m_{c}+m_{c}\right)\right.\right. \\
& \left.\left.\left.\left.\left.+\left(-a_{1}+a_{2}\right) m_{c} m_{h} r_{B / H_{y}}^{h}\right)\right)\right)\right)\right]
\end{aligned}
$$

$$
\begin{aligned}
& \ddot{q}_{3}=\left(a_{2} I_{h} m_{c}^{2} m_{h}{ }^{2} r_{B / C_{y}}^{c}\left(-a_{4}-\mathrm{c}\left(q_{3}+q_{4}\right) r_{B / C_{x}}^{c}+\mathrm{s}\left(q_{3}+q_{4}\right) r_{B / C_{y}}^{c}\right)\left(a_{9} r_{B / C_{x}}^{c}+a_{12} r_{B / H_{y}}^{h}\right)\right. \\
& +I_{c}^{2}\left(-m_{c} m_{h}\left(-a_{10} m_{h}+m_{c}\left(a_{7}+a_{5} m_{h}\right)\right) r_{B / H_{x}}^{h} r_{B / H_{y}}^{h}\left(-a_{3}+a_{4}+c\left(q_{3}\right) r_{B / H_{x}}^{h}\right.\right. \\
& \left.-\mathrm{s}\left(q_{3}\right) r_{B / H_{y}}^{h}\right)+I_{h}\left(m_{c}+m_{h}\right)\left(m_{h}\left(a_{9}+a_{11} r_{B / H_{x}}^{h}-a_{10} r_{B / H_{y}}^{h}\right)+m_{c}\left(a_{9}-\left(a_{8}\right.\right.\right. \\
& \left.\left.\left.\left.+a_{6} m_{h}\right) r_{B / H_{x}}^{h}+\left(a_{7}+a_{5} m_{h}\right) r_{B / H_{y}}^{h}\right)\right)\right)-I_{c} m_{c} m_{h}\left(a_{2} m_{c} m_{h}\left(a_{9} r_{B / C_{x}}^{c}+a_{12} r_{B / H_{x}}^{h}\right)\right. \\
& \times r_{B / H_{y}}^{h}\left(-a_{3}+a_{4}+\mathrm{c}\left(q_{3}\right) r_{B / H_{x}}^{h}-\mathrm{s}\left(q_{3}\right) r_{B / H_{y}}^{h}\right)+I_{h}\left(m _ { h } \left(a_{10} \mathrm{~s}\left(q_{3}+q_{4}\right) r_{B / C_{y}}^{c}{ }^{2} r_{B / H_{x}}^{h}\right.\right. \\
& -a_{2} r_{B / C_{y}}^{c}\left(a_{9}+a_{11} r_{B / H_{x}}^{h}\right)+a_{12}\left(a_{4} r_{B / H_{x}}^{h}-a_{2} r_{B / H_{y}}^{h}\right)+r_{B / C_{x}}^{c}\left(a_{4} a_{9}\right. \\
& \left.\left.-a_{10} c\left(q_{3}+q_{4}\right) r_{B / C_{y}}^{c} r_{B / H_{x}}^{h}+\left(-a_{4} a_{10}+a_{2} a_{11}\right) r_{B / H_{y}}^{h}\right)\right)+m_{c}\left(\mathrm{~s}\left(q_{3}+q_{4}\right)\right. \\
& \times\left(a_{7}+a_{5} m_{h}\right) r_{B / C_{y}}^{c}{ }^{2} r_{B / H_{x}}^{h}+a_{2} r_{B / C_{y}}^{c}\left(-a_{9}+\left(a_{8}+a_{6} m_{h}\right) r_{B / H_{x}}^{h}\right) \\
& +a_{12}\left(a_{4} r_{B / H_{x}}^{h}-a_{2} r_{B / H_{y}}^{h}\right)+r_{B / C_{y}}^{c}\left(a_{4} a_{9}+c\left(q_{3}+q_{4}\right)\left(a_{7}+a_{5} m_{h}\right) r_{B / C_{y}}^{c} r_{B / H_{x}}^{h}\right. \\
& \left.\left.\left.\left.+\left(a_{4} a_{7}-a_{2} a_{8}+\left(a_{4} a_{5}-a_{2} a_{6}\right) m_{h}\right) r_{B / H_{y}}^{h}\right)\right)\right)\right) \\
& /\left(-a_{2} I_{h}{ }^{2} m_{c}{ }^{2} m_{h}{ }^{2} r_{B / C_{x}}^{c} r_{B / C_{y}}^{c}\left(a_{4}+c\left(q_{3}+q_{4}\right) r_{B / C_{x}}^{c}-s\left(q_{3}+q_{4}\right) r_{B / C_{y}}^{c}\right)\right. \\
& +I_{c}^{2}\left(I_{h}{ }^{2}\left(m_{c}+m_{h}\right)^{2}+I_{h} m_{c} m_{h}\left(m_{c}+m_{h}\right)\left(\left(a_{3}-a_{4}\right) r_{B / H_{x}}^{h}+\left(-a_{1}+a_{2}\right) r_{B / H_{y}}^{h}\right)\right. \\
& \left.+\left(a_{1}-a_{2}\right) m_{c}{ }^{2} m_{h}{ }^{2} r_{B / H_{x}}^{h} r_{B / H_{3}}^{h}\left(-a_{3}+a_{4}+c\left(q_{13}\right) r_{B / H_{x}}^{h}-s\left(q_{3}\right) r_{B / H_{3}}^{h}\right)\right) \\
& -I_{c} I_{h} m_{c} m_{h}\left(I_{h}\left(m_{c}+m_{h}\right)\left(a_{1} r_{B / C_{x}}-a_{2} r_{B / C_{4}}^{c}\right)+m_{c} m_{h}\left(-\left(a_{2}\left(a_{3}-a_{1}\right)+\left(a_{1}-a_{2}\right)\right.\right.\right. \\
& \left.\times c\left(q_{3}+q_{4}\right) r_{B / C_{x}}^{c}\right) r_{B / C_{y}}^{c} r_{B / H_{x}}^{h_{n}}+\left(a_{1}-a_{2}\right) s\left(q_{3}+q_{4}\right) c_{B / C_{y}}{ }^{2} r_{B / H_{x}}^{h} \\
& \left.\left.+r_{B / C_{3}}^{c} r_{B / H_{3}}^{h}\left(-a_{3} a_{4}+a_{2} a_{1}+a_{2}\left(q_{3}\right) r_{B / H_{x}}^{h_{h}}-a_{2} s\left(q_{3}\right) r_{B / H_{y}}^{h}\right)\right)\right)
\end{aligned}
$$




$$
\begin{aligned}
& \ddot{q}_{4}=\left(-I_{h} m_{c} m_{h} r_{B / C_{y}}^{c}\left(-a_{4}-c\left(q_{3}+q_{4}\right) r_{B / C_{x}}^{c}+s\left(q_{3}+q_{4}\right) r_{B / C_{y}}^{c}\right)\left(I _ { h } \left(-a_{10} m_{h}\right.\right.\right. \\
& \left.\left.+m_{c}\left(a_{7}+a_{5} m_{h}\right)\right) r_{B / C_{y}}^{c}+a_{1} m_{c} m_{h}\left(a_{9} r_{B / C_{x}}^{c}+a_{12} r_{B / H_{x}}^{h}\right)\right)-I_{c}^{2}\left(-m_{c} m_{h}\left(-a_{10} m_{h}\right.\right. \\
& \left.+m_{c}\left(a_{7}+a_{5} m_{h}\right)\right) r_{B / H_{x}}^{h} r_{B / H_{y}}^{h}\left(-a_{3}+a_{4}+c\left(q_{3}\right) r_{B / H_{x}}^{h}-\mathrm{s}\left(q_{3}\right) r_{B / H_{y}}^{h}\right)+I_{h}\left(m_{c}+m_{h}\right) \\
& \left.\times\left(m_{h}\left(a_{9}+a_{11} r_{B / H_{x}}^{h}-a_{10} r_{B / H_{y}}^{h}\right)+m_{c}\left(a_{9}-\left(a_{8}+a_{6} m_{h}\right) r_{B / H_{x}}^{h}+\left(a_{7}+a_{5} m_{h}\right) r_{B / H_{y}}^{h}\right)\right)\right) \\
& +I_{c}\left(I _ { h } { } ^ { 2 } ( m _ { c } + m _ { h } ) \left(m_{h}\left(a_{12}-a_{11} r_{B / C_{x}}^{c}+a_{10} r_{B / C_{y}}^{c}\right)+m_{c}\left(a_{12}+\left(a_{8}+a_{6} m_{h}\right) r_{B / C_{x}}^{c}\right.\right.\right. \\
& \left.\left.-\left(a_{7}+a_{5} m_{h}\right) r_{B / C_{y}}^{c}\right)\right)+a_{1} m_{c}^{2} m_{h}^{2}\left(a_{9} r_{B / C_{x}}^{c}+a_{12} r_{B / H_{x}}^{h}\right) r_{B / H_{y}}^{h}\left(-a_{3}+a_{4}\right. \\
& \left.+\mathrm{c}\left(q_{3}\right) r_{B / H_{x}}^{h}-\mathrm{s}\left(q_{3}\right) r_{B / H_{y}}^{h}\right)+I_{h} m_{c} m_{h}\left(m _ { h } \left(a_{10} \mathrm{~s}\left(q_{3}+q_{4}\right) r_{B / C_{y}}^{c}{ }^{2} r_{B / H_{x}}^{h}-r_{B / C_{y}}^{c}\left(a_{1} a_{9}\right.\right.\right. \\
& \left.+\left(-a_{3} a_{10}+a_{4} a_{10}+a_{1} a_{11}\right) r_{B / H_{x}}^{h}\right)+F_{k 6}\left(a_{3} r_{B / H_{x}}^{h}-a_{1} r_{B / H_{y}}^{h}\right)+r_{B / C_{x}}^{c} a_{9} \\
& \left.\left.-a_{10} c\left(q_{3}+q_{4}\right) r_{B / C_{y}}^{c} r_{B / H_{x}}^{h}-\left(a_{4} a_{10}-a_{1} a_{11}+a_{10} c\left(q_{3}\right) r_{B / H_{x}}^{h}\right) r_{B / H_{y}}^{h}+a_{10} \mathrm{~s}\left(q_{3}\right) r_{B / H_{y}}^{h}{ }^{2}\right)\right) \\
& +m_{c}\left(-\mathrm{s}\left(q_{3}+q_{4}\right)\left(a_{7}+a_{5} m_{h}\right) r_{B / C_{y}}^{c}{ }^{2} r_{B / H_{x}}^{h}+r_{B / C_{y}}^{c}\left(-a_{1} a_{9}+\left(-a_{3} a_{7}+a_{4} a_{7}+a_{1} a_{8}\right.\right.\right. \\
& \left.+\left(-a_{3} a_{5}+a_{4} a_{5}+a_{1} B_{5}\right) m_{h}\right) r_{B / H_{x}}^{h}+a_{12}\left(a_{3} r_{B / H_{x}}^{h}-a_{1} r_{B / H_{y}}^{h}\right)+r_{B / C_{x}}^{c}\left(a_{3} a_{9}\right. \\
& +\mathrm{c}\left(q_{3}+q_{4}\right)\left(a_{7}+a_{5} m_{h}\right) r_{B / C_{y}}^{c} r_{B / H_{x}}^{h}+\left(a_{4} a_{7}-a_{1} a_{8}+a_{7} c\left(q_{3}\right) r_{B / H_{x}}^{h}\right. \\
& \left.\left.+m_{h}\left(a_{4} a_{5}-a_{1} a_{6}+a_{5} \mathrm{c}\left(q_{3}\right) r_{B / H_{x}}^{h}\right)\right) r_{B / H_{y}}^{h}+m_{h}\left(a_{4} a_{5}-a_{1} a_{6}+a_{5} \mathrm{c}\left(q_{3}\right) r_{B / H_{x}}^{h}\right)\right) r_{B / H_{y}}^{h} \\
& \left.\left.\left.\left.\left.-\mathrm{s}\left(q_{3}\right)\left(a_{7}+a_{5} m_{h}\right) r_{B / C_{y}}^{c}\right)\right)\right)\right)\right) \\
& /\left(-a_{2} I_{h}{ }^{2} m_{c}{ }^{2} m_{h}{ }^{2} r_{B / C_{x}}^{c} r_{B / C_{y}}^{c}\left(a_{4}+c\left(q_{3}+q_{4}\right) r_{B / C_{x}}^{c}-\mathrm{s}\left(q_{3}+q_{4}\right) r_{B / C_{y}}^{c}\right)\right. \\
& +I_{c}^{2}\left(I_{h}^{2}\left(m_{c}+m_{h}\right)^{2}+I_{h} m_{c} m_{h}\left(m_{c}+m_{h}\right)\left(\left(a_{3}-a_{4}\right) r_{B / H_{x}}^{h}+\left(-a_{1}+a_{2}\right) r_{B / H_{y}}^{h}\right)\right. \\
& \left.+\left(a_{1}-a_{2}\right) m_{c}^{2} m_{h}^{2} r_{B / H_{x}}^{h} r_{B / H_{y}}^{h}\left(-a_{3}+a_{4}+c\left(q_{3}\right) r_{B / H_{x}}^{h}-s\left(q_{3}\right) r_{B / H_{y}}^{h}\right)\right) \\
& -I_{c} I_{h} m_{c} m_{h}\left(I_{h}\left(m_{c}+m_{h}\right)\left(a_{4} r_{B / C_{x}}^{c}-a_{2} r_{B / C_{y}}^{c}\right)+m_{c} m_{h}\left(-\left(a_{2}\left(a_{3}-a_{4}\right)+\left(a_{1}-a_{2}\right)\right.\right.\right. \\
& \left.\times c\left(q_{3}+q_{4}\right) r_{B / C_{x}}^{c}\right) r_{B / C_{y}}^{c} r_{B / H_{3:}}^{h}+\left(a_{1}-a_{2}\right) s\left(q_{3}+q_{4}\right) r_{B / C_{y}}^{c}{ }^{2} r_{B / H_{x}}^{h} \\
& \left.\left.\left.+r_{B / C_{y}}^{c} r_{B H H_{3}}^{h_{h}}\left(-a_{1} a_{4}+a_{2} a_{4}+a_{2} c\left(q_{3}\right) r_{B / H_{x}}^{h}-a_{2} \mathrm{~s}\left(q_{3}\right) r_{B / H_{y}}^{h}\right)\right)\right)\right)
\end{aligned}
$$


Expressions for the reaction forces at the castor joint are:

$$
\begin{aligned}
F_{x}= & -\left(I _ { c } I _ { h } \left(m _ { c } m _ { h } \left(I _ { h } \left(m_{h}\left(a_{2} a_{12}+\left(a_{4} a_{10}-a_{2} a_{11}\right) r_{B / C_{x}}^{c}\right)+m_{c}\left(a_{2} a_{12}+\left(-a_{4} a_{7}\right.\right.\right.\right.\right.\right. \\
& \left.\left.\left.\left.+a_{2} a_{8}+\left(-a_{4} a_{5}+a_{2} a_{6}\right) m_{h}\right) r_{B / C_{x}}^{c}\right)\right)+\left(a_{2} a_{3}-a_{1} a_{4}\right) m_{c} m_{h}\left(a_{9} r_{B / C_{x}}^{c}+a_{12} r_{B / H_{x}}^{h}\right)\right) \\
& +I_{c}\left(I_{h}\left(m_{c}+m_{h}\right)\left(-a_{10} m_{h}+m_{c}\left(a_{7}+a_{5} m_{h}\right)\right)+m_{c} m_{h}\left(m _ { h } \left(\left(a_{1}-a_{2}\right) a_{9}\right.\right.\right. \\
& \left.+\left(-a_{3} a_{10}+a_{4} a_{10}+a_{1} a_{1 I}-a_{2} a_{11}\right) r_{B / H_{x}}^{h}\right)+m_{c}\left(\left(a_{1}-a_{2}\right) a_{9}\right. \\
& \left.\left.\left.\left.+\left(a_{3} a_{7}-a_{4} a_{7}-a_{1} a_{8}-a_{2} a_{8}+\left(a_{3} a_{5}-a_{4} a_{5}-a_{1} a_{6}+a_{2} a_{6}\right) m_{h}\right) r_{B / H_{x}}^{h}\right)\right)\right)\right) \\
/ & \left(-a_{2} I_{h}^{2} m_{c}^{2} m_{h}^{2} r_{B / C_{x}}^{c} r_{B / C_{y}}^{c}\left(a_{4}+c\left(q_{3}+q_{4}\right) r_{B / C_{x}}^{c}-\mathrm{s}\left(q_{3}+q_{4}\right) r_{B / C_{y}}^{c}\right)\right. \\
& +I_{c}^{2}\left(I_{h}^{2}\left(m_{c}+m_{h}\right)^{2}+I_{h} m_{c} m_{h}\left(m_{c}+m_{h}\right)\left(\left(a_{3}-a_{4}\right) r_{B / H_{x}}^{h}+\left(-a_{1}+a_{2}\right) r_{B / H_{y}}^{h}\right)\right. \\
& \left.+\left(a_{1}-a_{2}\right) m_{c}^{2} m_{h}^{2} r_{B / H_{x}}^{h} r_{B / H_{y}}^{h}\left(-a_{3}+a_{4}+c\left(q_{3}\right) r_{B / H_{x}}^{h}-\mathrm{s}\left(q_{3}\right) r_{B / H_{y}}^{h}\right)\right) \\
& -I_{c} I_{h} m_{c} m_{h}\left(I_{h}\left(m_{c}+m_{h}\right)\left(a_{4} r_{B / C_{x}}^{c}-a_{2} r_{B / C_{y}}^{c}\right)+m_{c} m_{h}\left(-\left(a_{2}\left(a_{3}-a_{4}\right)+\left(a_{1}-a_{2}\right)\right.\right.\right. \\
& \left.\times c\left(q_{3}+q_{4}\right) r_{B / C_{x}}^{c}\right) r_{B / C_{y}}^{c} r_{B / H_{x}}^{h}+\left(a_{1}-a_{2}\right) \mathrm{s}\left(q_{3}+q_{4}\right) r_{B / C_{y}}^{c}{ }^{2} r_{B / H_{x}}^{h} \\
& \left.\left.+r_{B / C_{y}}^{c} r_{B / H_{y}}^{h}\left(-a_{1} a_{4}+a_{2} a_{4}+a_{2} c\left(q_{3}\right) r_{B / H_{x}}^{h}-a_{2} \mathrm{~s}\left(q_{3}\right) r_{B / H_{y}}^{h}\right)\right)\right)
\end{aligned}
$$

$$
\begin{aligned}
F_{y}= & \left(m_{c} m_{h}\left(a_{2} a_{12} I_{c} m_{c} m_{h}+I_{c}\left(\left(a_{1}-a_{2}\right) a_{9} m_{c} m_{h}+I_{h}\left(-a_{10} m_{h}+m_{c}\left(a_{7}+a_{5} m_{h}\right)\right)\right)\right)\right. \\
& \times\left(I_{h} r_{B / C_{y}}^{c}\left(c\left(q_{3}+q_{4}\right) r_{B / C_{x}}^{c}-\mathrm{s}\left(q_{3}+q_{4}\right) r_{B / C_{y}}^{c}\right)+I_{c} r_{B / H_{y}}^{h}\left(c\left(q_{3}\right) r_{B / H_{x}}^{h}-\mathrm{s}\left(q_{3}\right) r_{B / H_{y}}^{h}\right)\right) \\
& -\left(\left(-a_{3}+a_{4}\right) a_{9} I_{c} m_{c} m_{h}+I_{h}\left(-a_{4} a_{12} m_{c} m_{h}+I_{c}\left(a_{11} m_{h}-m_{c}\left(a_{8}+a_{6} R m_{h}\right)\right)\right)\right) \\
& \left.\times\left(a_{2} I_{h} m_{c} m_{h} r_{B / C_{y}}^{c}+I_{c}\left(I_{h}\left(m_{c}+m_{h}\right)+\left(-a_{1}+a_{2}\right) m_{c} m_{h} r_{B / H_{y}}^{h}\right)\right)\right) \\
/ & \left(m _ { c } ^ { 2 } m _ { h } ^ { 2 } ( a _ { 2 } I _ { h } r _ { B / C _ { x } } ^ { c } + ( - a _ { 1 } + a _ { 2 } ) I _ { c } r _ { B / H _ { x } } ^ { h } ) \left(I_{h} r_{B / H_{y}}^{h}\left(c\left(q_{3}+q_{4}\right) r_{B / C_{2}}^{c}-\mathrm{s}\left(q_{3}+q_{4}\right) r_{B / C_{y}}^{c}\right)\right.\right. \\
& \left.+I_{c} r_{B / H_{y}}^{h}\left(c\left(q_{3}\right) r_{B / H_{x}}^{h}-a_{2} s\left(q_{3}\right) r_{B / H_{y}}^{h}\right)\right)-\left(-a_{4} I_{h} m_{c} m_{h} r_{B / C_{x}}^{c}+I_{c}\left(I_{h}\left(m_{c}+m_{h}\right)\right.\right. \\
& \left.\left.+\left(a_{3}-a_{4}\right) m_{c} m_{h} r_{B / H_{y}}^{h}\right)\right)\left(a_{2} I_{h} m_{c} m_{h} r_{B / C_{y}}^{c}+I_{c}\left(I_{h}\left(m_{c}+m_{h}\right)+\left(-a_{1}\right.\right.\right. \\
& \left.\left.\left.\left.+a_{2}\right) m_{c} m_{h} r_{B / H_{y}}^{h}\right)\right)\right)
\end{aligned}
$$

